\title{
A Probabilistic Evaluation of Earthquake Detection and Location Capability for Illinois, Indiana, Kentucky, MASTER Ohio, and West Virginia
}

Prepared by F. J. Mauk, D. H. Christensen

Department of Geological Sciences

University of Michigan

Prepared for

U.S. Nuclear Regulatory

Commission 


\section{DISCLAIMER}

This report was prepared as an account of work sponsored by an agency of the United States Government. Neither the United States Government nor any agency Thereof, nor any of their employees, makes any warranty, express or implied, or assumes any legal liability or responsibility for the accuracy, completeness, or usefulness of any information, apparatus, product, or process disclosed, or represents that its use would not infringe privately owned rights. Reference herein to any specific commercial product, process, or service by trade name, trademark, manufacturer, or otherwise does not necessarily constitute or imply its endorsement, recommendation, or favoring by the United States Government or any agency thereof. The views and opinions of authors expressed herein do not necessarily state or reflect those of the United States Government or any agency thereof. 


\section{DISCLAIMER}

Portions of this document may be illegible in electronic image products. Images are produced from the best available original document. 


\section{The following pages are an exact representation of what is in the original document folder.}




\title{
NOTICE
}

This report was prepared as an account of work sponsored by an agency of the United States Government. Neither the United States Government nor any agency thereof, or any of their employees, makes any warranty, expressed or implied, or assumes any legal liability or responsibility for any third party's use, or the results of such use, of any information. apparatus product or process disclosed in this report, or represents that its use by such third party would not infringe privately owned rights.

\section{DO NOT MICROFILM COVER}

\author{
Available from \\ GPO Sales Program \\ Division of Technical Information and Document Control \\ U. S. Nuclear Regulatory Commission \\ Washington, D. C. 20555 \\ Printed copy price: $\$ 6.00$ \\ and \\ National Technical Information Service \\ Springfield, Virginia 22161
}


NUREG/CR-1648 R6, RA

\section{A Probabilistic Evaluation of Earthquake Detection and Location Capability for Illinois, Indiana, Kentucky, Ohio, and West Virginia}

NUREG/CR--1648

TI86 900266

Manuscript Completed: June 1980

Date Published: September 1980

Prepared by

F. J. Mauk, D. H. Christensen

Seismological Observatory

Department of Geological Sciences

University of Michigan

Ann Arbor, MI 48109

Prepared for

Division of Reactor Safety Research

Office of Nuclear Regulatory Research

U.S. Nuclear Regulatory Commission

Washington, D.C. 20555

NRC FIN No. B5545

Under Contract No. NRC-04-76-192 

Probabilistic estimations of earthquake detection and location capabilities for the states of Illinois, Indiana, Kentucky, Ohio and West Virginia are presented in this document. The algorithm used in these epicentrality and minimum-magnitude estimations is a version of the program NETWORTH by Wirth, Blandford, and Husted (DARPA Order No. $2551,1978)$ which was modified for local array evaluation at the University of Michigan Seismological Observatory. Estimations of earthquake detection capability for the years 1970 and 1980 are presented in four regional minimum $m_{b}$ magnitude contour maps: Regional $90 \%$ confidence error ellipsoids are included for $m_{b}$ magnitude events from 2.0 through 5.0 at $0.5 \mathrm{~m}_{\mathrm{b}}$ unit increments. The close agreement between these predicted epicentral $90 \%$ confidence estimates and the calculated error ellipses associated with actual earthquakes within the studied region suggest that these error determinations can be used to estimate the reliability of epicenter location.

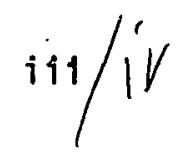


Probabilistic estimations of earthquake detection and location capabilities for the states of Illinois, Indiana, Kentucky, Ohio and West Virginia are presented in this document. The algorithm used in these epicentrality and minimum magnitude estimations is a version of the program NETWORTH by Wirth, Blandford, and Husted (DARPA Order No. 2551, 1978). Estimations of earthquake detection capability for the years 1970 and 1980 are presented in four regional minimum $m_{b}$ magnitude contour maps. The network for 1970 consists of 33 known active seismograph stations in eastern North America; for 1980, 157 of the 276 known active stations are used. All near regional stations are used, regardless of their performance record, to insure that the coverage is representative. The attenuation function used is derived from Rịchter. (1936) and Eyernden (1967) for the eastern United States. The trayel-time data used are from Herrin (1968), Evernden (1967), and Arnold (1968). The required signal to noise ratio is set at 1.5 . Regịonal $90 \%$ confidence error ellipsoids are included for $\mathrm{m}_{b}$ magnitude events from 2.0 through 5.0 at $0.5 \mathrm{~m}_{\mathrm{b}}$ unit increments. The minimum station detectability criterion is set at 10 for events with magnitudes greater than 2.5 and 5 for eyents with magnitudes less than 2.5. The close agreement between these predicted epiçentral $90 \%$ confidence estimates and the calculated error ellipses associated with actual earthquakes wịthin the studied region suggest that these error determinations can be used to estimate the the performance of a seismograph station nelwur'k in the absence of ahundant earthquakes.

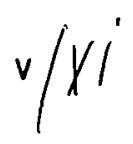


Abstract

$i i j$

Summary

Introduction

The Transfer Functions

The Station Network

Analysis of Earthquake Detection and Location Capabilities for Illinois, Indiana, Kentucky, Ohio, and West Virginia for 1970 and 1980

References

Appendix Ia Eastern North America Seismograph 35 Stations

Appendix Ib Seismograph Station Operation Code 43 and Telephone Number

Appendix Ic Criteria for Assigning Noise and

Reliability Estimates for Seismograph Stations

Appendix IIa Error Ellipse Parameters $m_{b} 5.0 \quad 48$

Appendix IIb Error Ellipse Parameters $m_{b} 4.5 \quad 55$

Appendix IIc Error Ellipse Parameters $m_{b} 4.0 \quad 62$

Appendix IId Error E1lipse Parameters $m_{b} 3.5 .69$

Appendix IIe Error Ellipse Parameters $m_{b} 3.0 \quad 76$

Appendix IIf Error Ellipse Parameters $m_{b} 2.5 \quad 83$ 10 station coverage

Appendix IIg Error Ellipse Parameters $m_{b} 2.590$ 5 station coverage

Appendix IIh Error Ellipse Parameters $m_{b} 2.0 \quad 97$

Appendix III NETWORTH ALGORITHM 104

$$
\text { vii/VIIl }
$$


Figure 1. - Ground displacement attenuation curves for, $m_{1}$ magnitude 2.0-5.5 earthquakes (after Evernden 1967)

Figure 2: - Active seismograph stations for eastern North America in 1980 which were used for network analys is

Figure 3. - Five station magnitude detection threshold in 1970

Figure 4. - Ten station magnitude detection threshold in $1970 \quad 19$

Figure .5. - Five station magnitude detection threshold in 20 1980

Figure 6. - Ten station magnitude detection threshold in $1980 \quad 21$

Figure. 7. - $90 \%$ Confidence error ellipses for $m_{b} 5.0$ évents 22 10 station minimum cöverage

Figure 8. - $90 \%$ Confidence error ellipses for $m_{b} 4.5$ events 10 station minimum coverage

Figure 9. - $90 \%$ Confidence error ellipses for $m_{b} 4.0$ events 10 station minimum coverage

Figure 10. - 90\% Confidence error ellipses for $m_{b} 3.5$ events 10 station minimum coverage

Figure 11. - 90\% Confidence error éllipses for $m_{b} 3.0$ events 26 10 station minimum coverage

Figure 12. - $90 \%$ Confidence error ellipses for $m_{b} 2.5$ events 10 station minimum coverage

Figure 13. - $90 \%$ Confidence error ellipses for $m_{b} 2.5$ events

Figure 14. - $90 \%$ Confidence error ellipses for $m_{b} 2.0$ events

\section{TABLES}

Table 1. - P-wave attenuation coefficients as a function of distance

Table 2. - Herrin 1968 P-wave travel time table for surface focus events

Table 3. - Standard deviation of travel time data as a function of distance 
$\therefore \quad$ INTRODUCTION

During the past decade, both the U. S. Nuclear Regulatory Commission and the U. S. Geological Survey have been involved actively in programs to improve the evaluation of earthquake hazard and risk in the United States east of the Rocky Mountains. With few exceptions, the historic seismic data base for the eastern United States is not adequate to locate accurately the hypocenters of many moderately large earthquakes nor to provide significant estimations of possible recurrence rates. One method of attacking this problem has been to increase the number of seismograph stations in the eastern United States; particularly emphasizing regions known to have experienced dammaging earthquakes historically. - In the north-central region of the United States, for example, there were twenty-three active regional sefsmic observatories in 1970. By 1980, this number had grown to over 100. The intent of this observatory proliferation is twofold: first to enhance significantly the hypocenter location capabilities, ultimately defining seismically active geological structures, and secondly to depress the earthquake magnitude detection threshold, thereby providing a broader data base for rational evaluation of recurrence rates. The actual effectiveness of the four-fold increase in the number of observatories in the north-central region of the United States has not been examined, however. In fact, prior to 1970 , 1ittle attention was paid to the eastern United States earthquake detection capabilities other than a few preliminary investigations during the LRSM program of the VELA Uniform Project of the early 1960's. This report is a probabilistic evaluation of earthquake detection and location capabilities for the north-central region of the United States, particularly the states of Illinois; Indiana, Kentucky, Ohio, and West Virginia. 
The basic algorithm used in this report to evaluate the capabilities of a network of seismograph stations was written by Wirth, Blandford and Husted (1978, DARPA Order No. 2551). Alterations of the algorithm to accomodate a larger number of stations, more refined station input parameters and buffering to interactive graphics routines were done at the University of Michigan Seismological Observatory in 1980. A copy of the code is included as an appendix to this report.

Estimates of both minimum magnitude detection probability and epicentrality precision are computed from the same input data. Three types of Information are required in these evaluations; epicenter parameters, transfer functions, and station parameters. The epicenter parameters are the coordinates of the locations to be evaluated either at fixed $m_{b}$ magnitudes or at a minimum magnitude determined by the distribution and performance of the stations. The transfer functions are the regional travel-time and attenuation curves. The station parameters include locations and instrumental performance characteristics.

A station is assumed to detect an event provided that the ratio of signal to noise amplitude is greater than some fixed value, $r$. We have assumed the value of $r$ to be 1.5 in the evaluations included in this report. Signal and noise are assumed to be log-normally distributed. If the $\log$ of the noise amplitude has expectation $\mu_{n}$ and variance $\sigma_{n}^{2}$ and the $\log$ of the signal amplitude has expectation $\log A$ and variance $\sigma_{S}^{2}$, then the probability that a station $i$ will detect an event is given by:

$$
P_{i}=P_{i}(\Phi)\left[\frac{\log A-\left(\mu_{n}+\log r\right)}{\left\{\sigma_{n}^{2}+\sigma_{s}^{2}\right\}^{1 / 2}}\right]_{i}
$$


where $P_{j}$ is an estimation of the reliability of operation of station $i$ where $0 \leq P_{i} \leq 1.0$ and

$$
\Phi(x)=\int_{-\infty}^{x} e^{-y^{2} / 2} d y / \sqrt{2 \pi}
$$

is the normal cumulative probability function. The values for $P_{i}, \mu_{n}$, and $\sigma_{n}^{2}$ for each station are empirically derived from examination of seismograms from each station. The value of $\log A$ is derived from

$$
\log _{10} A_{i}=m_{b}+b_{\Delta}
$$

where $m_{b}$ is the $P$-wave magnitude and $b_{\Delta}$ is the attenuation coefficient for distance $\Delta$. The attenuation coefficient table will be discussed later in this report.

Given $N$ independent events with probability $P_{i}$, the probability of at least $\alpha$ of the events is given by (Booker, 1964) as:

$$
P(\geq \alpha)=\sum_{k=\alpha}^{N} p(k)
$$

where

$$
p(k)=E_{k}(p)-\left(\begin{array}{c}
k+l \\
k
\end{array}\right) E_{k+1}(p)+\left(\begin{array}{c}
k+2 \\
k
\end{array}\right) E_{k+2}(p)-\ldots \pm\left(\begin{array}{c}
N \\
k
\end{array}\right) E_{N}(p)
$$

is the probability of exactly $k$ events, and

$$
E_{1}(p)=\sum_{i=1}^{N} P_{i} ; E_{2}(p)=\sum_{i>j}^{N} p_{i} p_{j} ; E_{3}(p)=\sum_{i>j>k}^{N} p_{i} p_{j} p_{k} ;
$$

etc.

The value of $\alpha$ used in our calculations is usually 10 unless otherwise stated and the minimum acceptable probability of detection is 0.9 . In 
other words, we define our minimum detection acceptability as $90 \%$ probability of detection by at least ten stations.

The determination of hypocentral parameters, latitude longitude depth and origin time, from arrival times is a non-linear problem which generally is addressed by utilizing a linearization and least squares iterative reduction of arrival time residuals as defined by Geiger (1910). The exactness of this solution is dependent on the precision with which the arrival times at the stations can be picked, the precision and variance of the empirically derived regional travel time curves, and the distribution of seismograph stations about the location of interest. The confidence regions constructed in the linearized problem become exact asymptotically as the number of stations utilized becomes large. and are approximately ellipsoidal in the neighborhood of the mean estimated location provided that they have semimajor axes with lengths not exceeding 20 kilometers (Flinn, 1964). Confidence regions determined with small numbers of stations which are poorly distributed or having semimajor axcs lengths excéeding 20 k1lometers may deyiate significantly from ellipsoidal shape and may not accurately represent hypocentral uncertainty.

Al though the NETWORTH algorithm has the capability of using several regional variations of both travel-time data and attenuation characteristics, we have assumed uniform travel-time and attenuation functions throughout the region. Thus the absolute accuracy of detection capabilities within the Mississippi Embayment for example, where the attenuation function is acknowledged to differ from that for the remainder of the eastern United States, may be marginally different than indicated in this report. 
THE TRANSFER FUNCTIONS

The two transfer functions required to evaluate seismograph network performance are the distance dependent P-wave attenuation coefficients and the P-wave travel-times. Since we are interested in the ground displacements at given distances, the geometrical spreading and anelastic attenuation effects are combined in a table of logarithmic decrements to the $m_{b}$ magnitude as a function of distance (See táble 1 ). These attenuation coefficients are derived from a variety of sources and form a piece-wise continuous attenuation function. The coefficients for the distance range 0 through $200 \mathrm{kilometers}\left(1.8^{\circ}\right)$ are assumed to be due purely to geometrical spreading and the function is constrained to pass through the $100 \mathrm{kilometer}$ distance ground amplitude value as defined by Richter (1937). This distance range is the most difficult to define accurately since it includes the most pronounced perturbations due to local structure and:non-linear finite displacement effects. For these reasons, the absolute accuracy of the computed displacements at near-field distances is questionable and likely underestimation of the actual ground displacements. Fortunately, however, these near-field inaccuracies play a relatively insignificant role in the evaluation of regional network performance since the observational thresholds for the majority of the stations will be outside this distance range. It is important to recognize, however, that, $m_{b}$ magnịtudes calculated for epicentral distances less than 60 kilometers are overestimated due to the extrapolated positive values of the attenuation function in this distance range, regardless of near-field non-linear finite displacement effects. 
The attenuation coefficients for the distance range 200 kilometers through 1400 kilometers are derived from the Evernden (1967) eastern United States $P_{8.5}$ attenuation curve. There is a transition from the $P_{8.5}$ to the $P_{10.5}$ curve of Evernden in the distance range 1400 through 1600 kilometers. This transition is along the 'shot report' curve and serves to connect these disjoint segments of the attenuation function in a smooth manner. The attenuation function from 1600 kilometers to $45^{\circ}$ is a combination of the Evernden $\mathrm{P}_{10.5}$ and 'shot report' curves. The tabulated logarithmic decrements to the $m_{b}$ magnitudes as a function of distance used in this study are given in table 1. In addition, the ground displacements, in nanometers, as a function of distance for a range of $m_{b}$ magnitudes are illustrated in figure 1 .

The other transfer function utilized in network evaluation is the $\mathrm{P}$-wave travel time data. The mean distance-dependent $\mathrm{P}$-wave travel times are from Herrin (1968) for surface focus events. The distance increment is $0.1^{\circ}$ frnm $n$ to $11^{\circ}$ and $0.6^{\circ}$ bcyond $11^{\circ}$. The lravel times are for $P_{g}$ from 0 to $1.1^{\circ}, P^{*}$ from 1.1 to $1.8^{\circ}, P_{n}$ from 1.8 to $11^{\circ}$, and $P$ beyond $11^{\circ}$. These travel times are given in table 2.

The standard deyiations about the mean travel times as a function of distance are given in table 3. The coefficients in the distance range from 0 through $25^{\circ}$ were obtained from the data scatter for three explosions and three earthquakes in the eastern United States given by Evernden (1967). Standard deviations of the travel time curve beyond $25^{\circ}$ are from Arnold (1968). These data are used in the construction of the hypocentral error ellipsoids. 
Table 1.

Attenuation coefficients as $m_{b}$ magnitude decrements as a function of distance

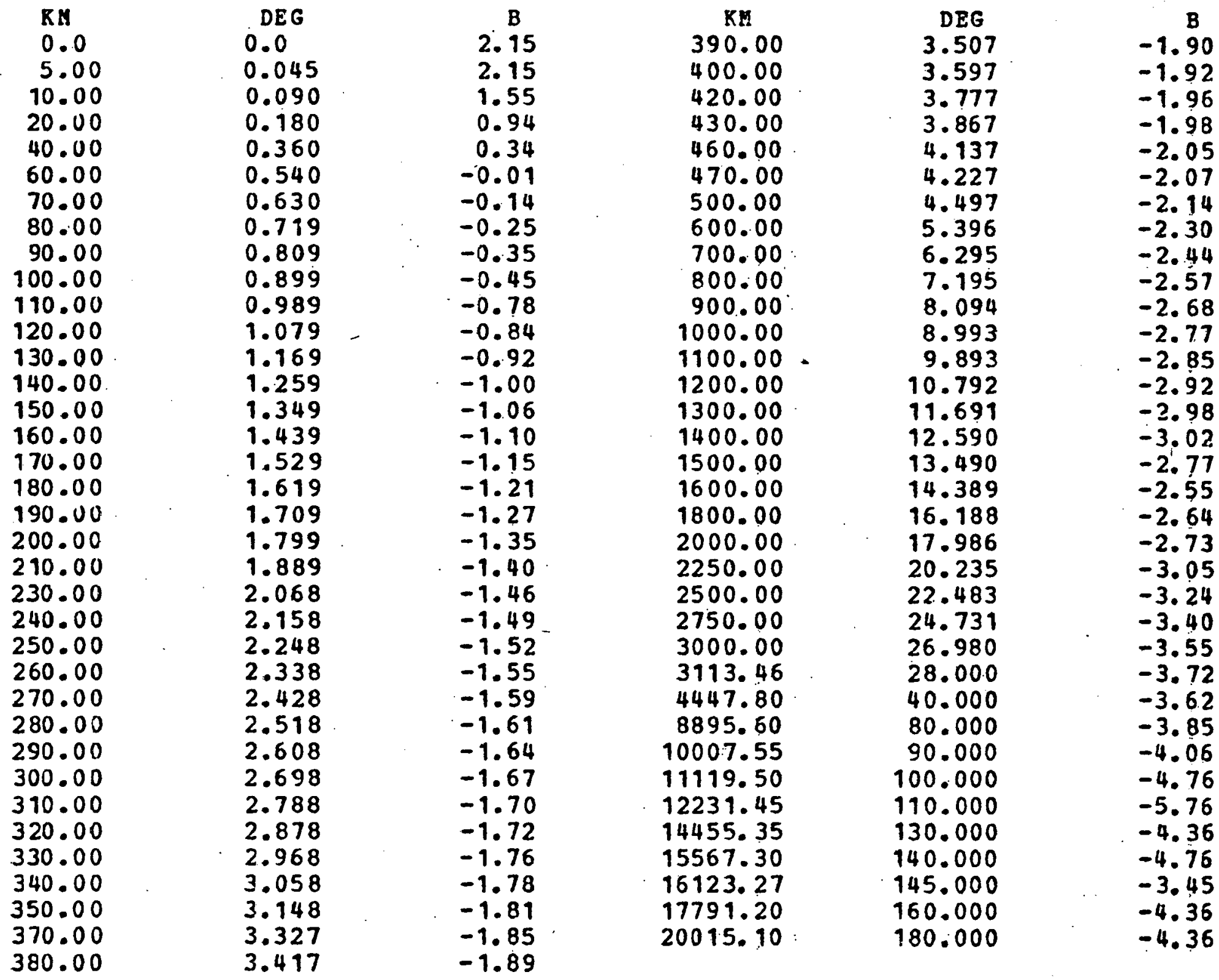


Figure 1.

$P$-Wave Attenuation

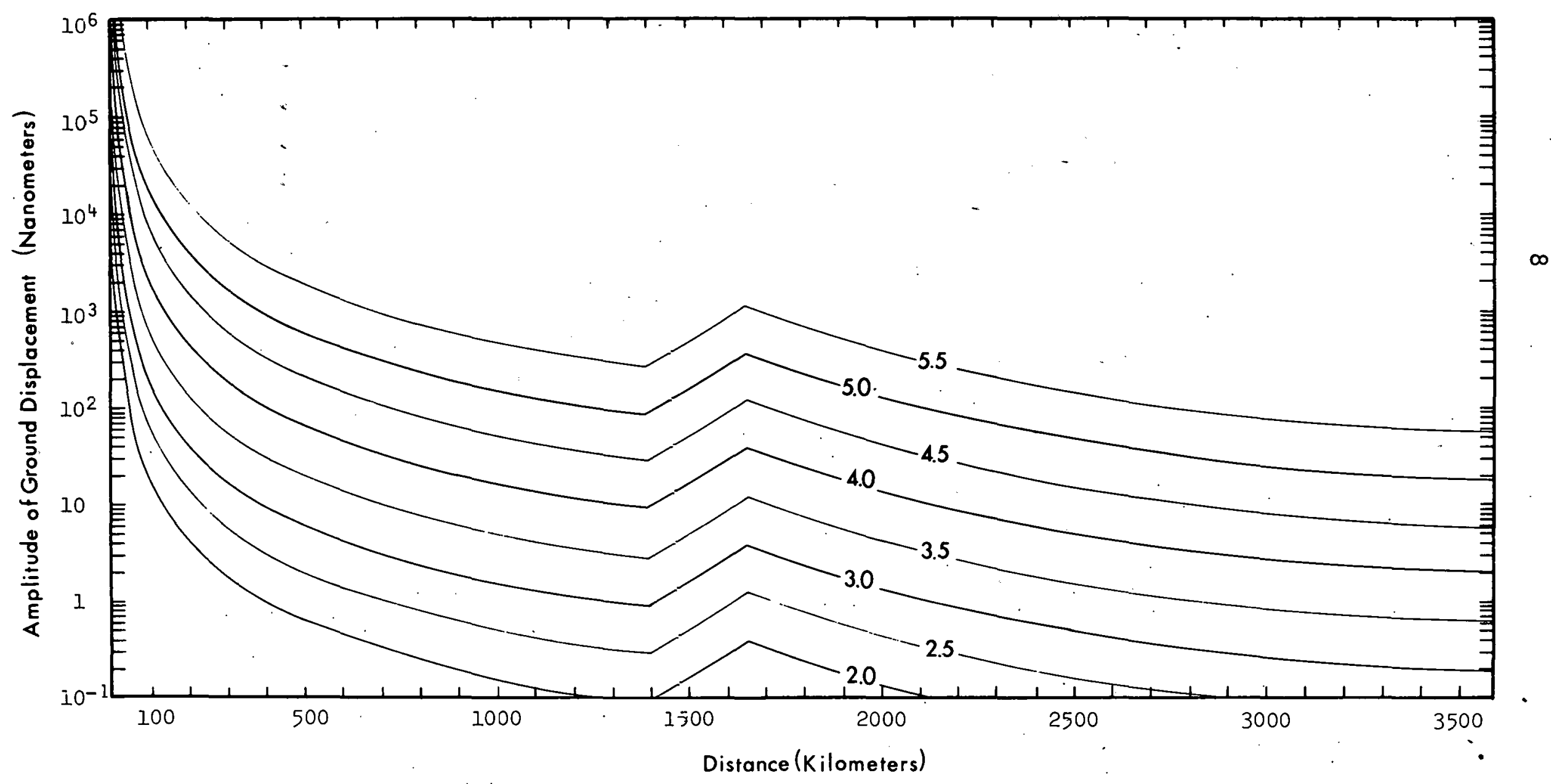


Table 2.

HERRIN6 8

TRAVEL-TIME. TABLE

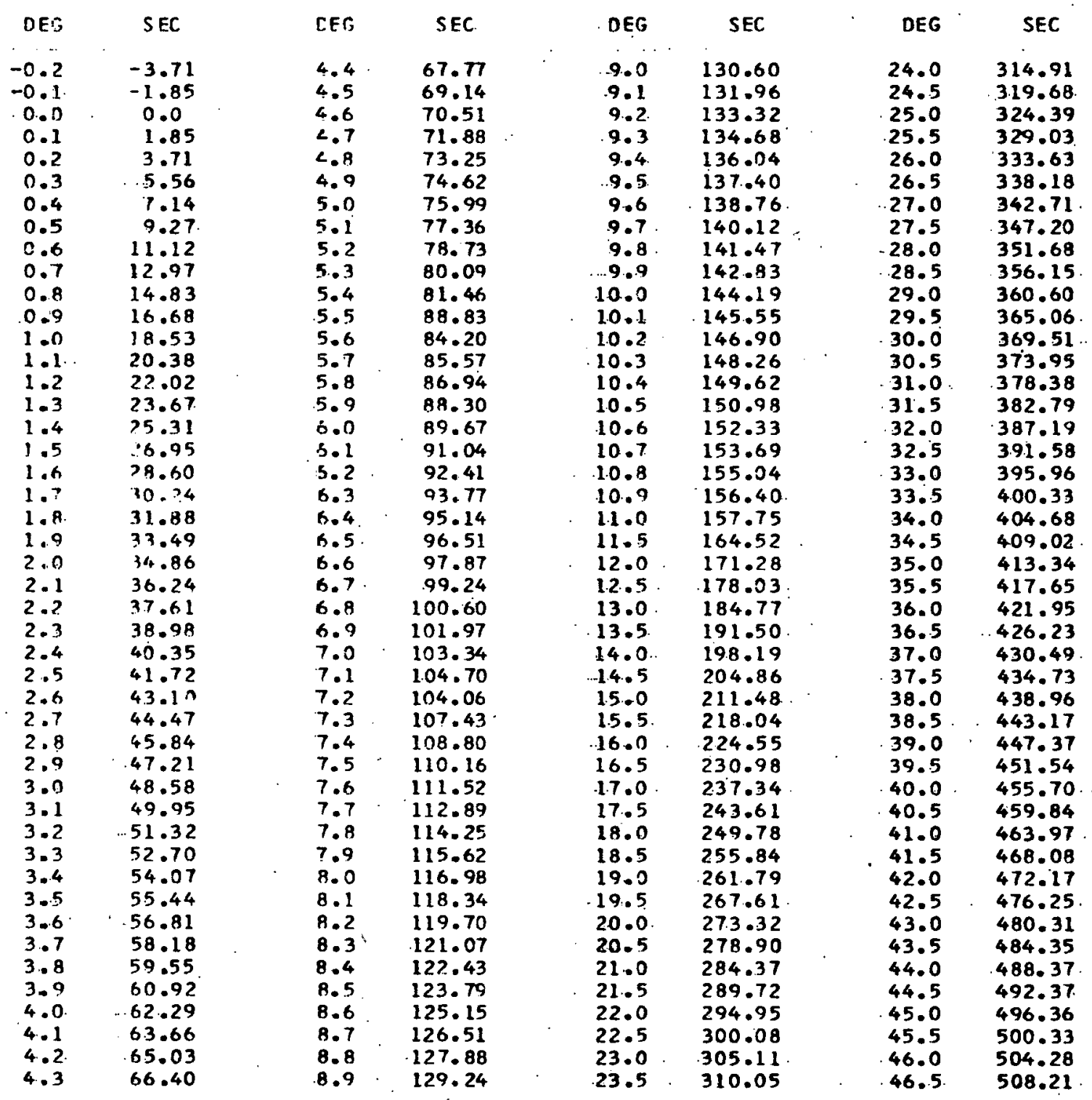




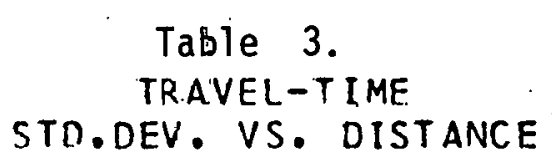

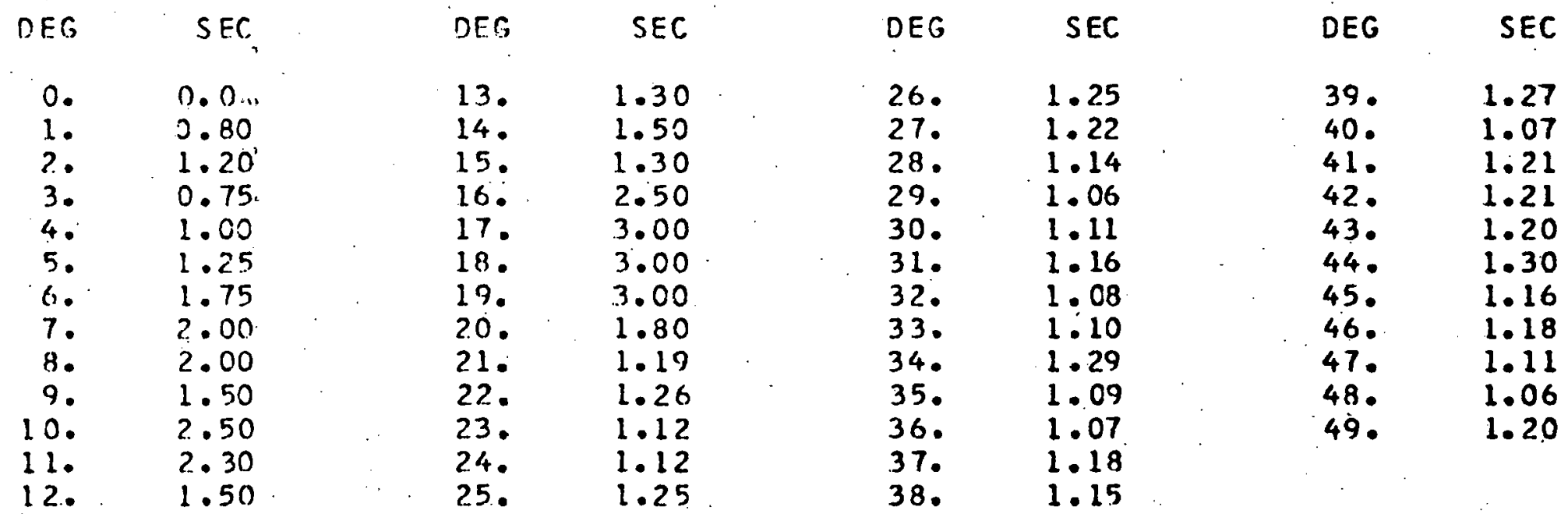




\section{THE STATION NETWORK}

The distribution of operational seismograph stations in eastern North America which were used in the evaluation of event detection and and location capabilities for the north-central region of the United States is illustrated in figure 2 . These stations are a subset of the total number of active observatories in eastern North America which are catalogued in appendix Ia. Although it would be desirable to use the entire catalog of active stations in 1980, it is neither practical nor reasonable to do so.

The network includes all near regional stations to insure that the coverage within the area of interest is accurately represented; particularly for small magnitude events. In addition, a well-distributed large number of far regional stations is included to prevent unrealistic edge effects and to insure that coverage of larger magnitude events is reasonably accurate. The network stations are indicated in appendix Ia by an asterix following their respective code letters. Other station parameters included in appendix Ia are: latitude, longitude, elevation above mean sea level in meters, state, a code indicating the organization responsible for station operation (See also appendix Ib), the peak magnification of the short-period vertical instrument and the center frequency, a characteristic rms background noise value in nanometers and code indicating how the noise value was assigned (See also appendix Ic), a station reliability estimation and code indicating assignment procedure, and an averago travel-time and $m_{b}$ magnitude bias estimation for the station, if it is known.

The two most important parameters defining event detection by a given 
station are the characteristic background noise level and the station reliability. Both of these parameters proved to be quite difficult to obtain in a standardized manner and a series of criteria were established for both parameters. The letter codes in Appendix Ia which follow the rms noise value and the reliability correspond to the method of assigning each of these parameters given in Appendix Ic. An assignment of 0 gain at 0 frequency in the table indicates that the parameters for that station could not be obtained.

In actuality, the ratio of the signal amplitude and the average amplitude of the noise at the same frequency is the event observational limitation for any given station. Without spectral amplitude functions of the noise at all of the, stations, however, this refined evaluation of detection capability is not currently possible. Alternatively, we have required a unfform signal to noise ratio of 1.5 for all stations, and defined the standard noise characteristic as the observational 7 imitation regardless of the frequency. The standard deviation of the noịse has been set at a yalue of $\pm 0.25 \mathrm{~m}_{b}$ units. 


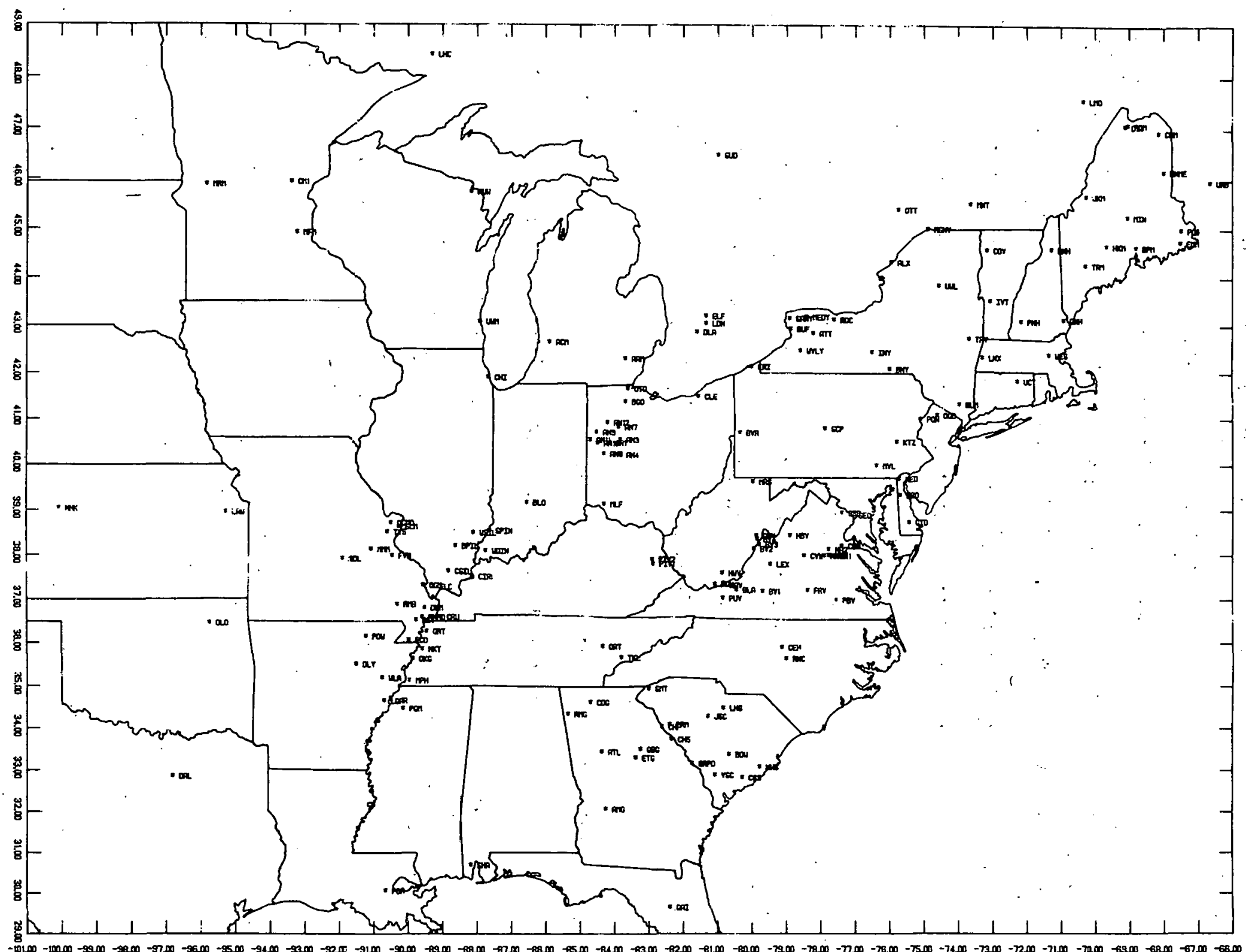

. Figure 2 
ANALYSIS OF EARTHQUAKE DETECTION AND LOCATION CAPABILITIES FOR ILLINOIS, INDIANA, KENTUCKY, OHIO, AND WEST VIRGINIA FOR 1970 AND 1980

Essentially two types of information are of interest in a detection capability study. First, what minimum magnitude earthquake is detectable at a given location by a specific station network. Second, what dimensions and orientations are associated with the location error ellipsoids surrounding specific hypocenters of given magnitude events. Both of these aspects are discussed in this report. Two levels of coverage are considered. The first is a five station minimum $90 \%$ detection probability threshold. This was considered to be a basic requirement since five stations barely overdetermine the hypocenter parameter matrix in the inverse problem. In addition, the error ellipsoids associated with such low level coverage are usually large. Thus, although five station coverage yields an estimation of the minimum magnitude event observable, the absolute hypocenter parameters in the inverse problem are not very reliable. Figures 3 and 4 illustrate the five station minimum $90 \%$ probability earthquake detection capability for the north central region of the United States in 1970 and 1980 respectively. The maps are contoured at $0.1 \mathrm{~m}_{\mathrm{b}}$ magnitude unit intervals and are based on 294 equally spaced $\left(0.5^{\circ}\right)$ grid points across the region. The improvement in regional detection capability from 1970 to 1980 is demonstrated by comparing the two contour maps. The regional earthquake detection threshold has been depressed by approximately one $m_{b}$ magnitude unit. The improvement in coverage of the three specific regional seismic zones is even more dramatic. The minimum $m_{b}$ magnitude earthquake observable in the Wabash Valley in 1970 was 3.8 as compared with 2.1 in 
1980. Similarly, the Anna, Ohio region had a minimum five station detection capability of $m_{b} 3.7$ in 1970 as compared with $m_{b} 1.1$ in 1980. The West Virginia-Virginia border also had an improvement in detectability from $m_{b} 3.8$ to $m_{b} 1.8$.

The majority of information presented in this document is for 10 station minimum detection. This threshold level more conservatively estimates the minimum magnitude earthquake observable, but simultaneously provides a more realistic evaluation of error ellipsoid parameters. Two minimum magnitude contour maps based on ten station minimum $90 \%$ probable detection are included as figures.5 and 6 for 1970 and 1980 respectively. By placing a 10 station 1 imit on the $90 \%$ probability of detection, arrays such as the ones in the Wabash Valley and Anna, Ohio are statistically overpowered since neither contains the minimum number of stations. This raises the minimum magnitude event contour for both regions to 2.7 and to 2.4 for the West Virginia-Virginia border. In addition, because of the thrce stations, AAM UTO and BGO, Lhe cuntour for magnitude 2.6 events is centered north of the Anna, Ohio region rather than sorrounding it.

The second aspect of the regional coverage to be considered is the absolute location accuracy for events of specified magnitude. Error ellipse information has been computed for events with magnitudes 5.0, $4.5,4.0,3.5,3.0$, and 2.5 having a 10 station minimum coverage threshold. In addition, error ellipse information for magnitude 2.5 and 2.0 events having a five station minimum coverage threshold are also included. The computéd hypocenter precision parameters are tabulated in appendix II and graphically illustrated in figures 7 through 14 . 
Data in Appendix II include the coordinates of the error ellipse center, the event magnitude, the number of stations included in the computation of the error ellipse, the largest open angle of station coverage, the dimensions of the error ellipse semimajor and semiminor axes, the angle of clockwise rotation of the semimajor axis from north, the area of the error ellipse, and the $95 \%$ confidence dimension of the depth determination. If the joint detection probability for the minimum station coverage $(5$ or 10$)$ is less than 0.9 then the error ellipse parameters are set to zero and the maximum joint probability of observation by the station network is listed in place of the magnitude.

The error ellipses are plotted to scale at each grid point on figures 7 through 14. If the error ellipse at any grid point has a semimajor axis length which exceeds 50 kilometers, only the center of the ellipse is plotted. Locations where the probability of detection criterion is not met remain blank on the figures.

Location accuracy is reasonably good for earthquakes with $m_{b}$ magnitudes 5.0 through 3.5 (Figures 7-10, Appendix IIa-IId). Semiaxes lengths rarely exceed. 7 kilometers and are typically 2 or 3 kilometers. Resolution of $m_{b} 3.0$ event epicenters (Figure 11, Appendix IIe) is significantly less accurate however. The majority of the north central United States region has nearly circular error ellipses with semiaxes lengths generally 5 to 7 kilometers. Central Indiana and western Illinois, on the other hand are characterized by high eccentricity error ellipses with semimajor axes lengths frequently exceeding 15 kilometers and orlented In a riurthwest-southeast dircction.

Two computations of $m_{b} 2.5$ error ellipses have been included. 


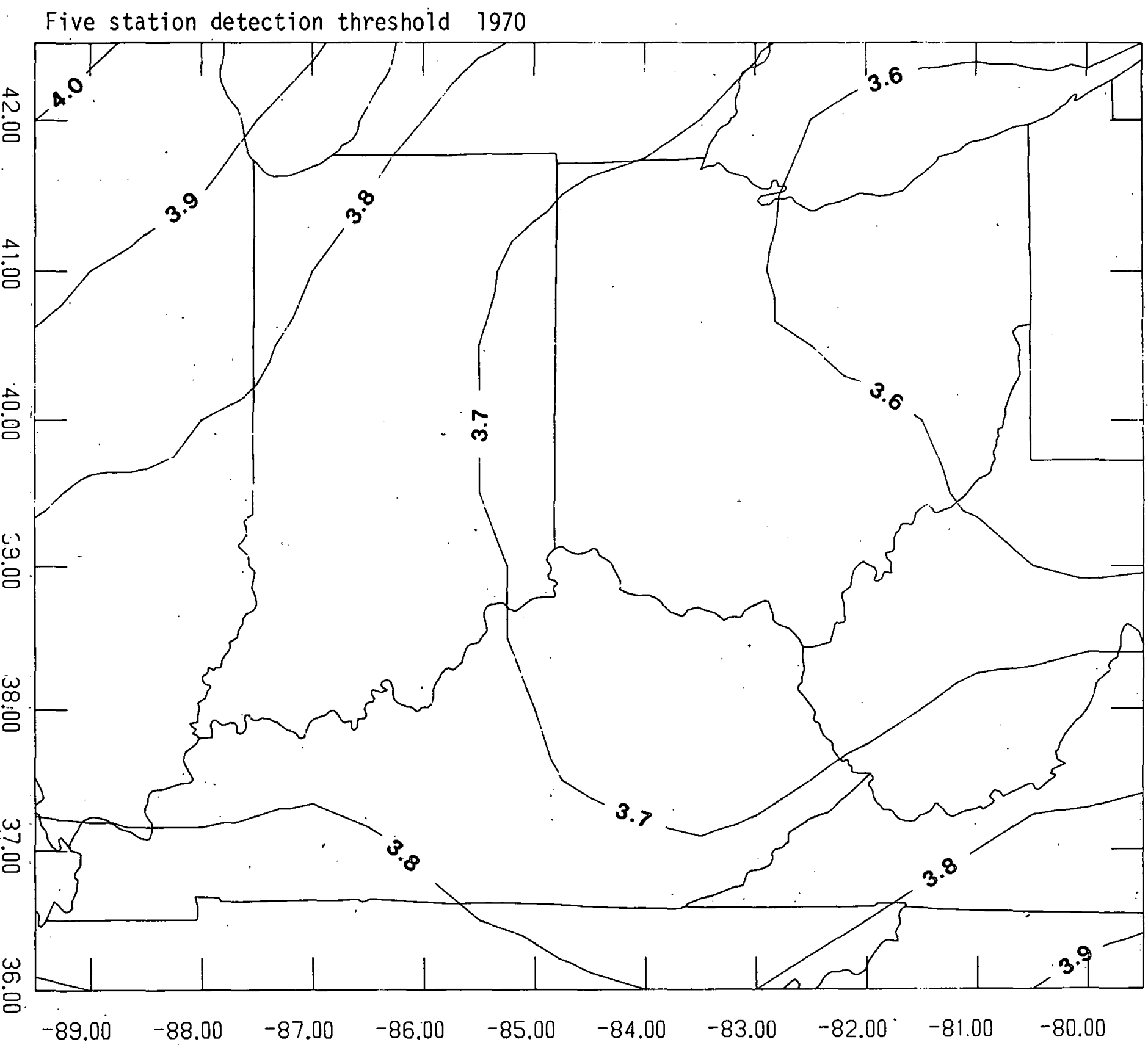

Figure 3 


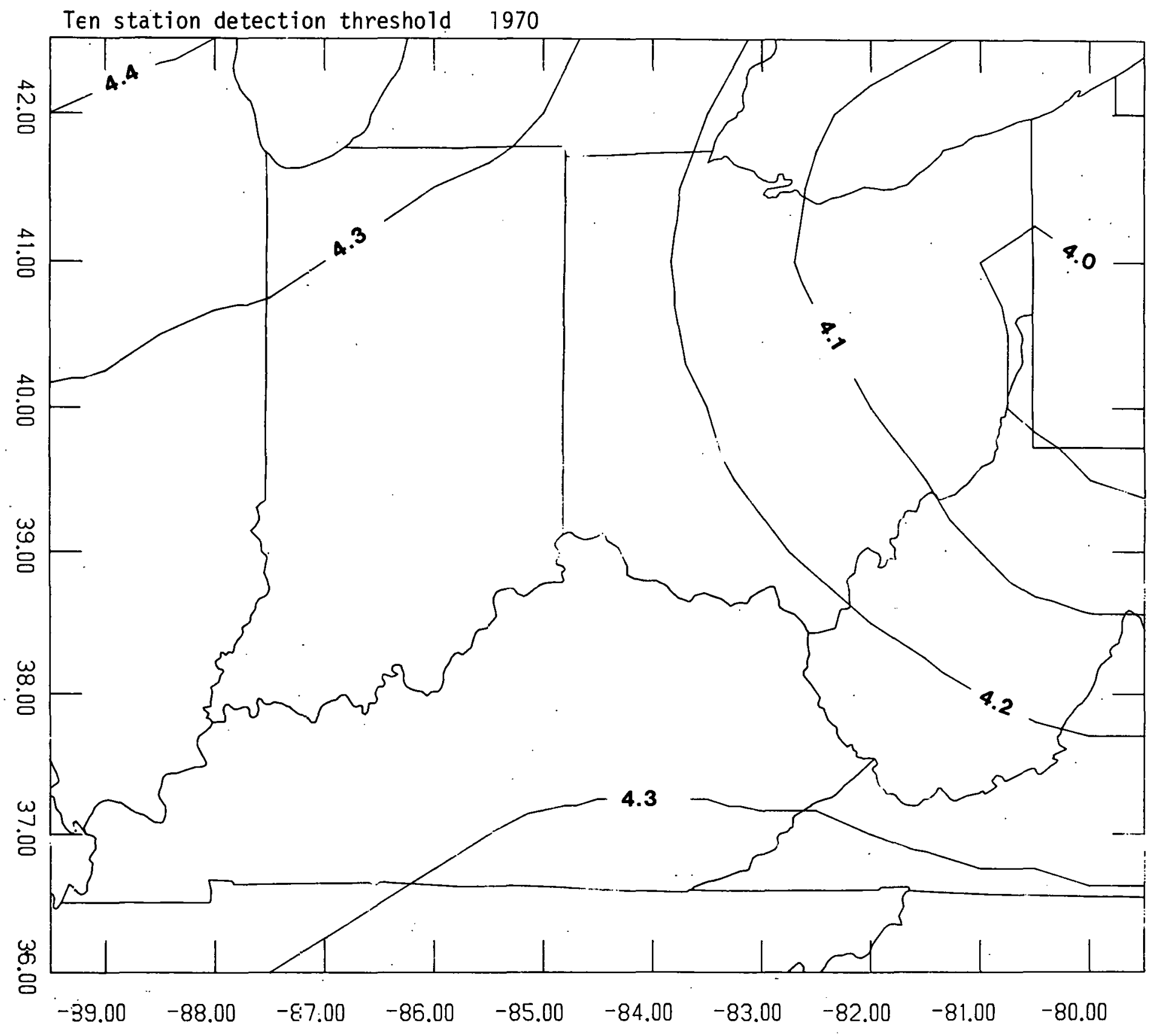

Figure 4 


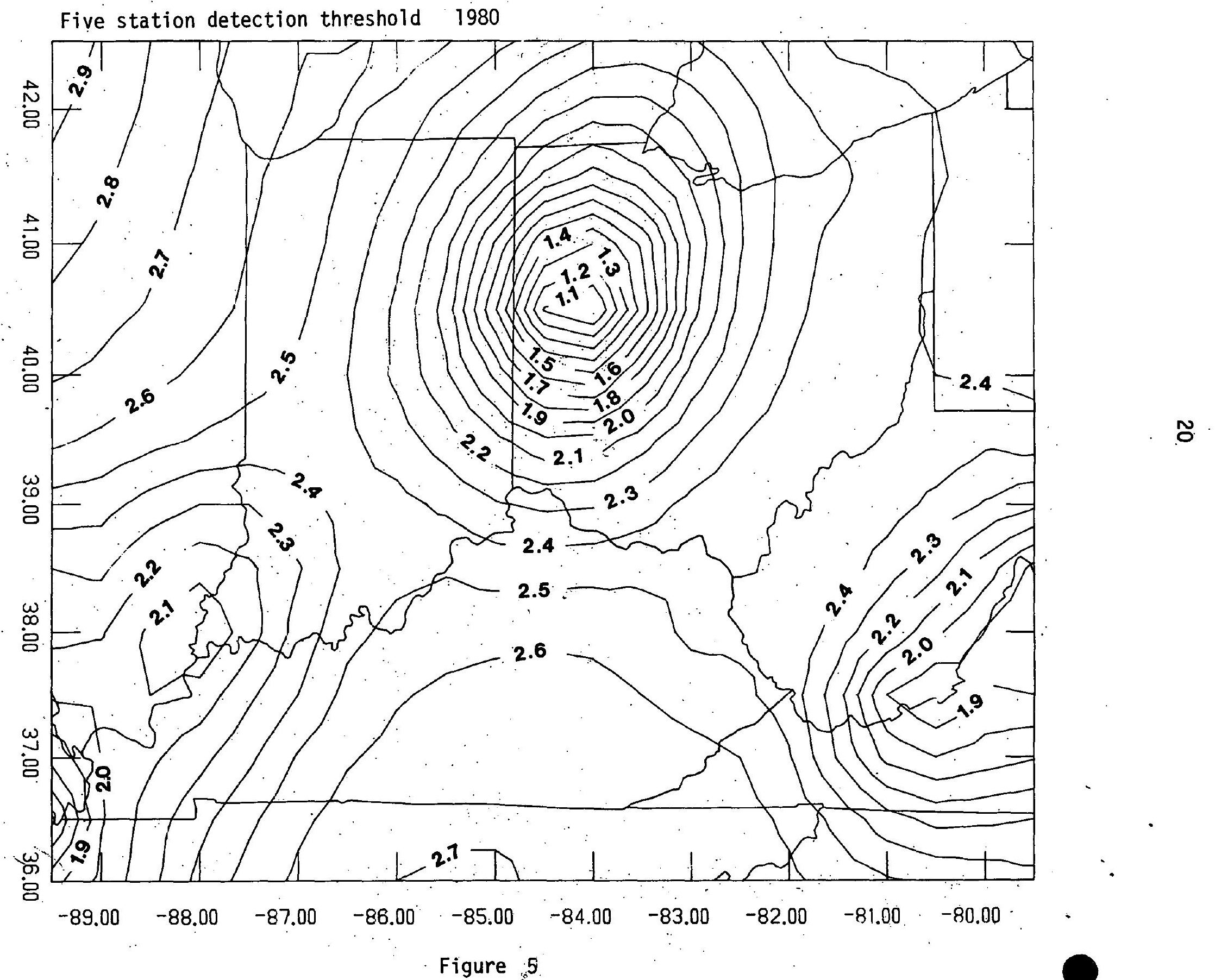




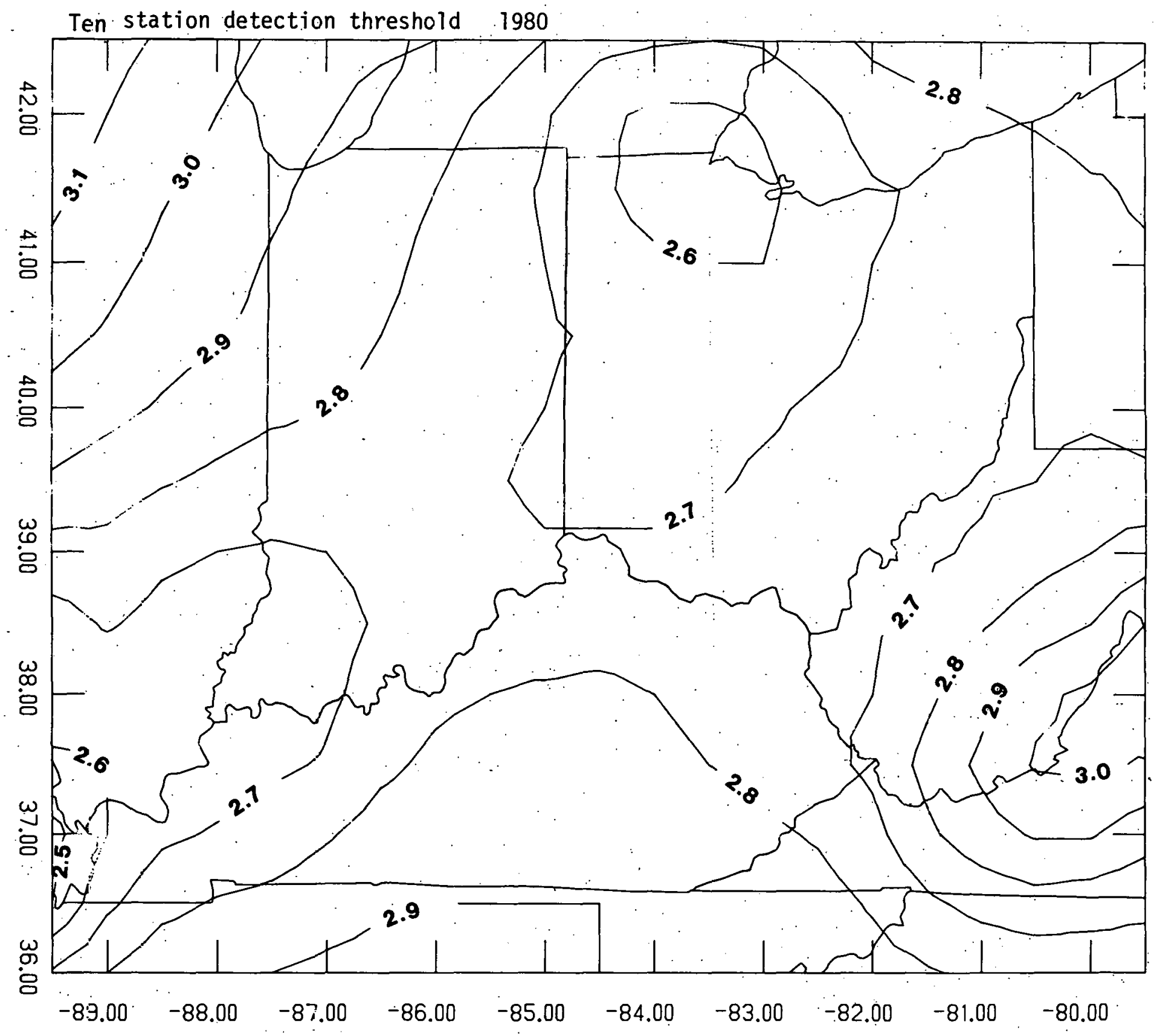

Figure 6 


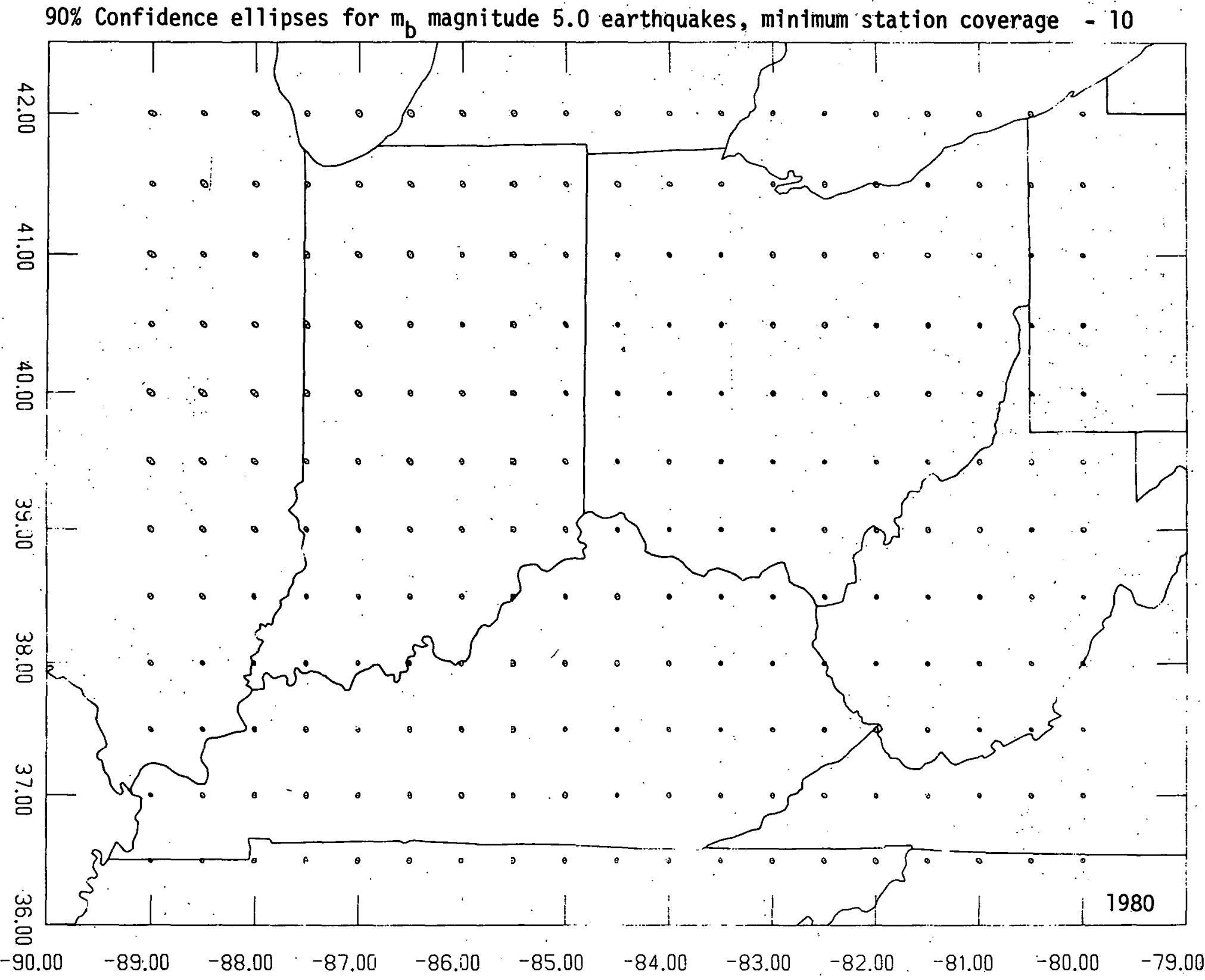

Figure 7 
$\varsigma_{\theta} \%$ Confidence ellipses for $m_{6}$ magnitude $4: 5$ earthquakes, minimum station coverage $\div 10$

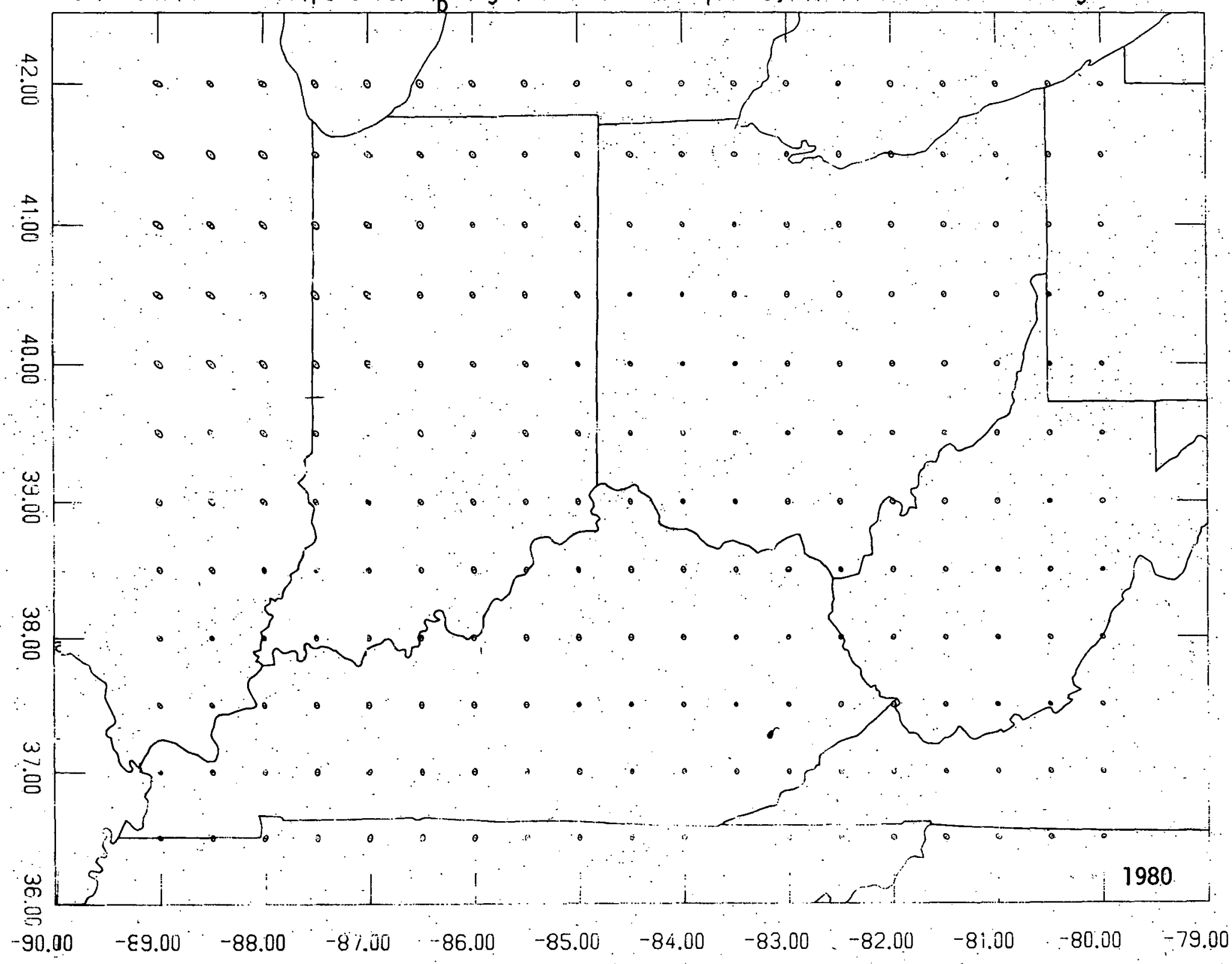


$90 \%$ Confidence ellipses for $m_{\mathrm{b}}$ magnitude $4: 0$ earthquakes, minimum station coverage -10

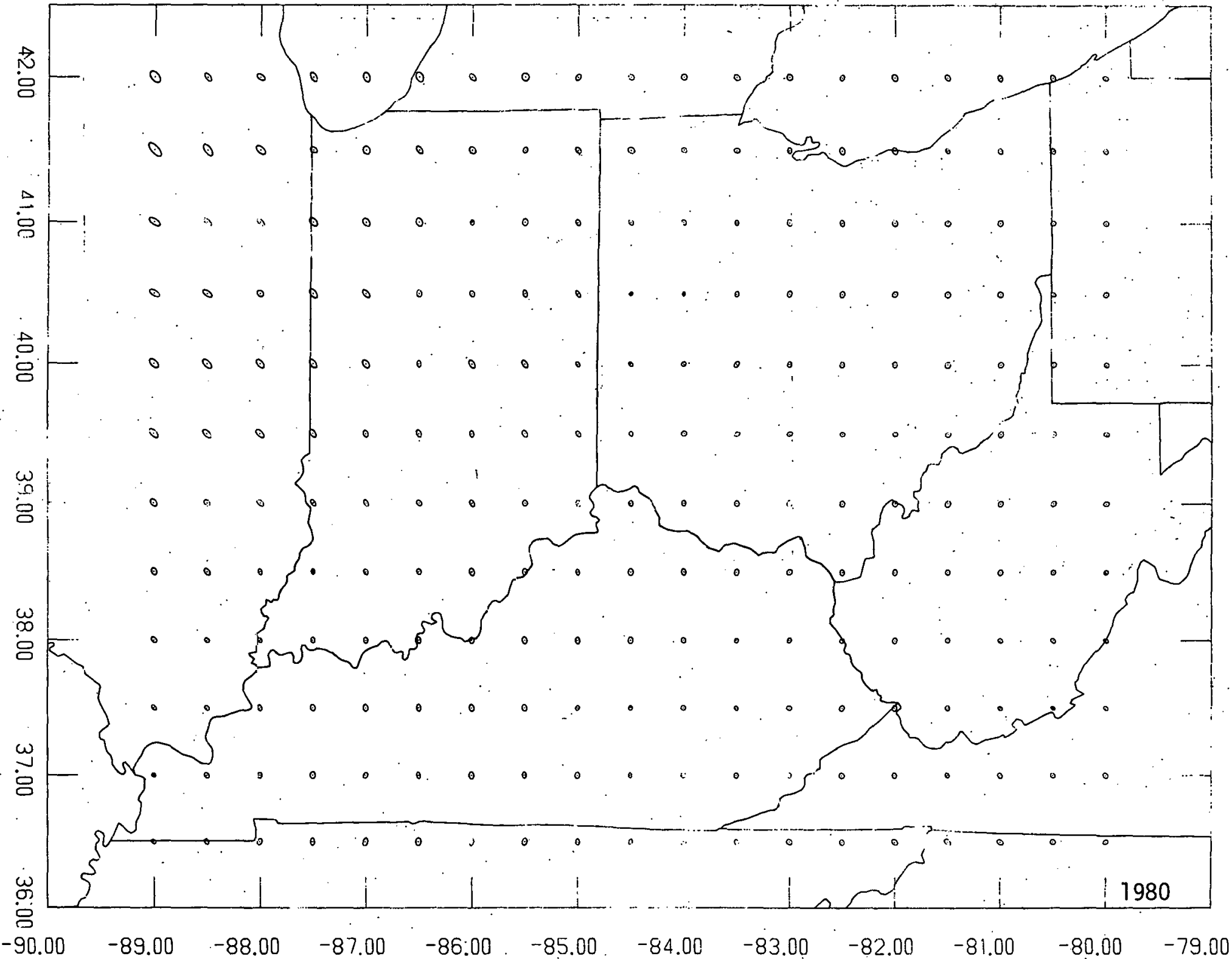


$90 \%$ Confidence ellipses for $m_{b}$ magnitude 3.5 earthquakes, minimum station coverage

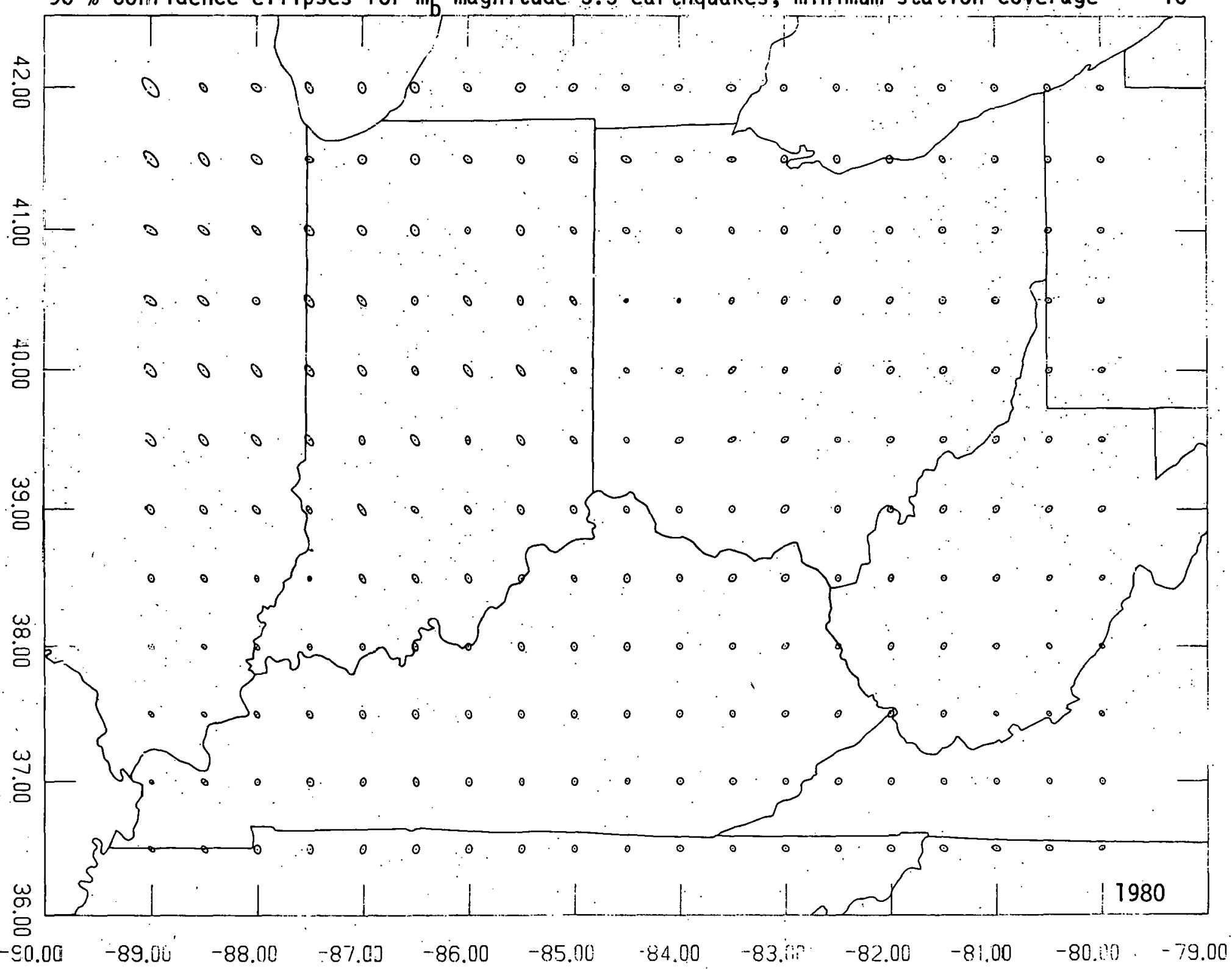


$90 \%$ Confidence ellipses for $m_{\mathrm{b}}$ magnitude 3.0 earthquakes, minimum station coverage -10

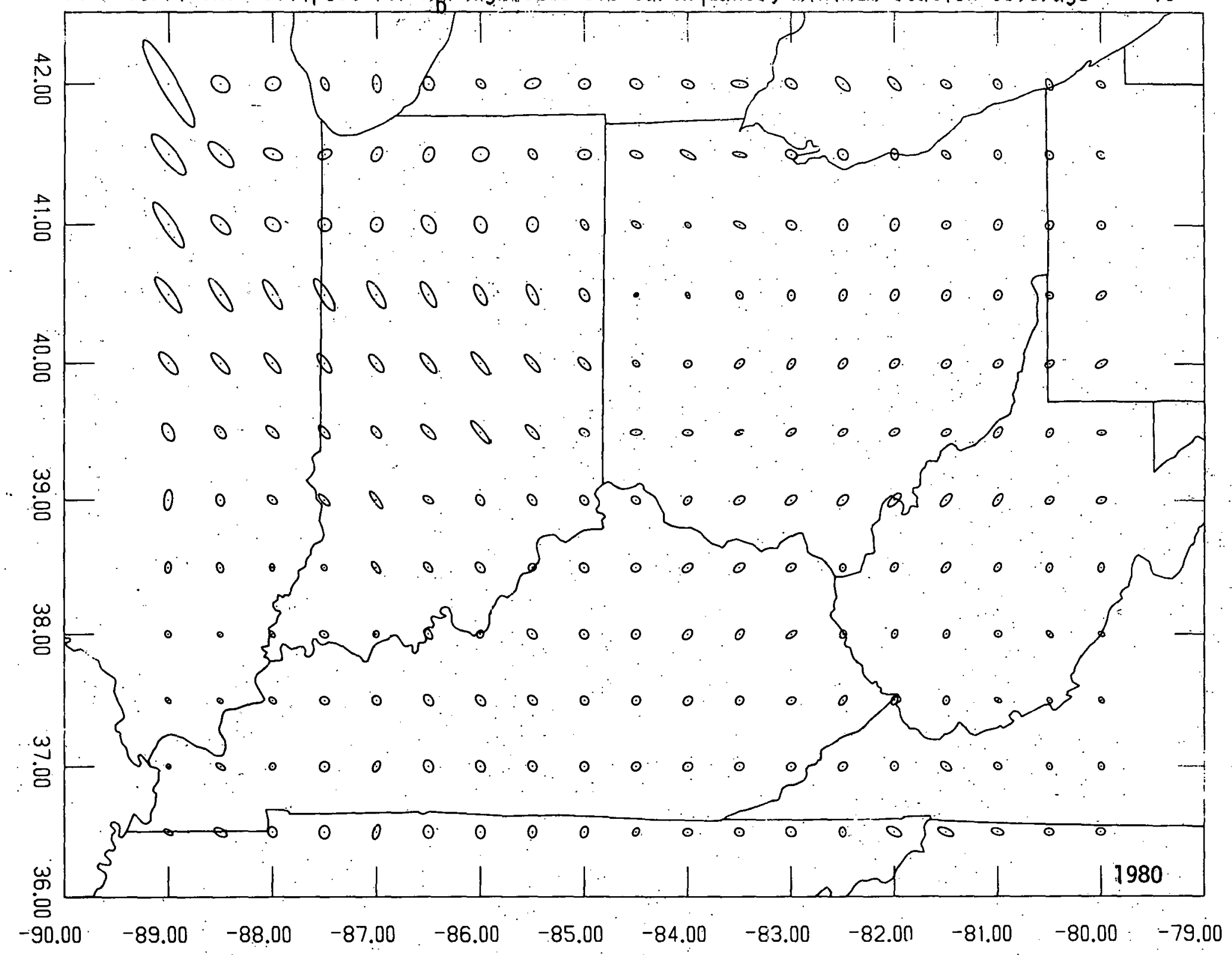


Figure 11 and Appendix IIf are those for a ten station minimum detection threshold, figure 12 and Appendix IIg are for a five station minimum detection threshold. The ground displacement for $m_{b}$ 2.5 earthquakes in the distance range 100 to $200 \mathrm{kilometers}$ is 65 to 15 nanometers (See figure 1). This is also the range of the average background noise characteristic for most stations in Appendix Ia. Thus $m_{b} 2.5$ events are at the fringe of interarray location capabilities for the north central region of the United States. In addition, since most of the eastern United States arrays consist of less than 10 elements, the five station detection threshold becomes more realistic for events less than or equal to $m_{b} 2.5$.

Error ellipses for magnitude 2.5 events in the north-central region of the United States are highly eccentric and frequently have semimajor axes exceeding 35 kilometers in length. Curiously, the distribution of stations constrains the trends of the semimajor axes of the error ellipses in strongly preferred northeast and northwest trends (See figure 12). This is also the case for $m_{b} 3.0$ events but to a less pronounced degree. The orientations of the semimajor:ares of the error ellipses at the treshold magnitudes in 1970 also show this tendency but the eccentricity of these ellipses is much less pronounced. These analyses suggest the possibility that the hypothesized northeast and northwest trends of seismicity in this region may not be geologically controlled, but may in fact be an artifact caused by the non-random distribution of seismograph stations. Thus a great deal of caution should be exercised in defining seismicity trends in these directions.

The final analysis presented is for error ellipses associated with 


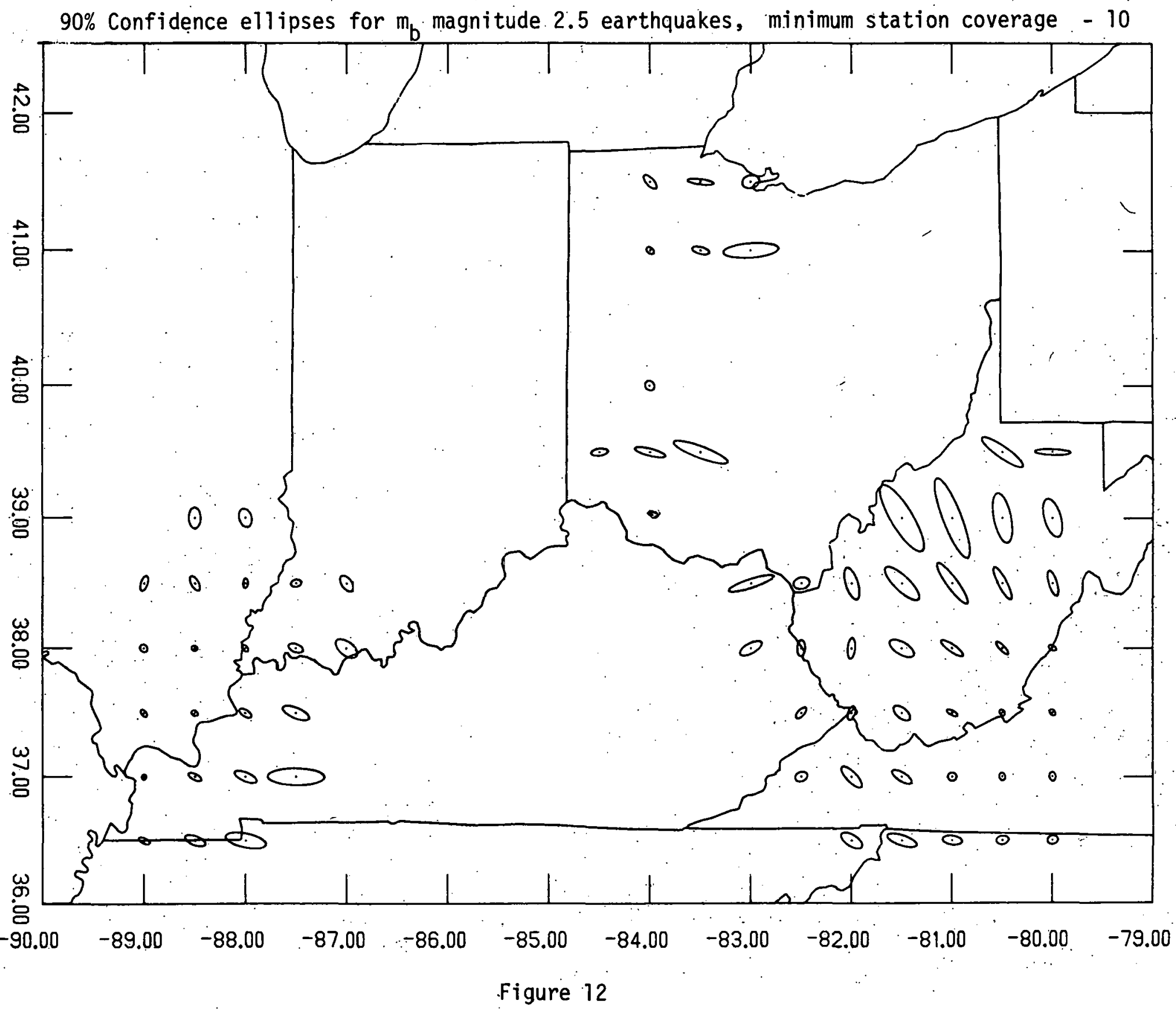


$90 \%$ Confidence ellipses for $m_{b}$ magnitude 2.5 earthquakes, minimum station coverage - 5

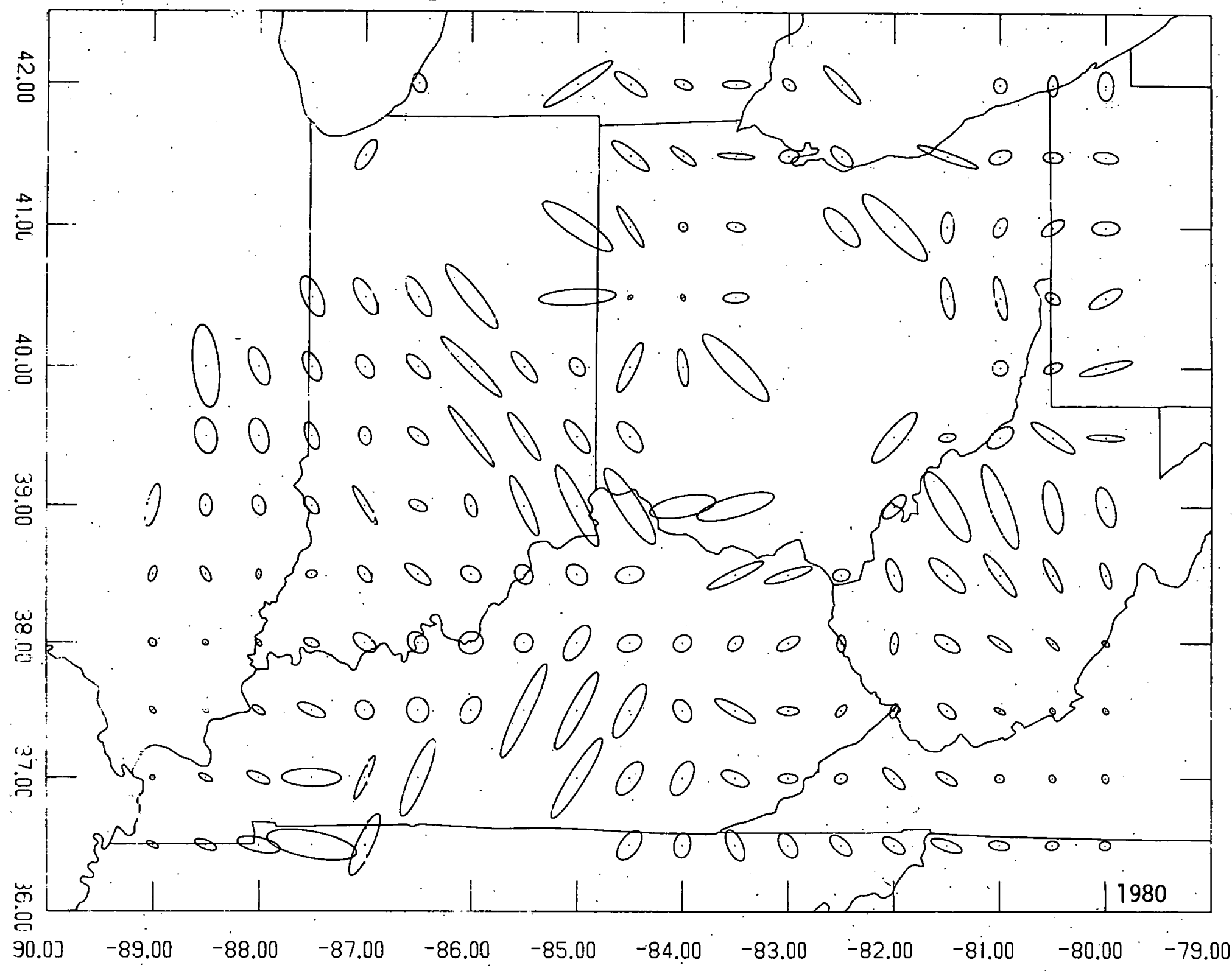


90. \% Confidence ellipses for $\mathrm{m}_{\mathrm{b}}$ magnjtude 2.0 earthquakes, minimum station coverage - 5

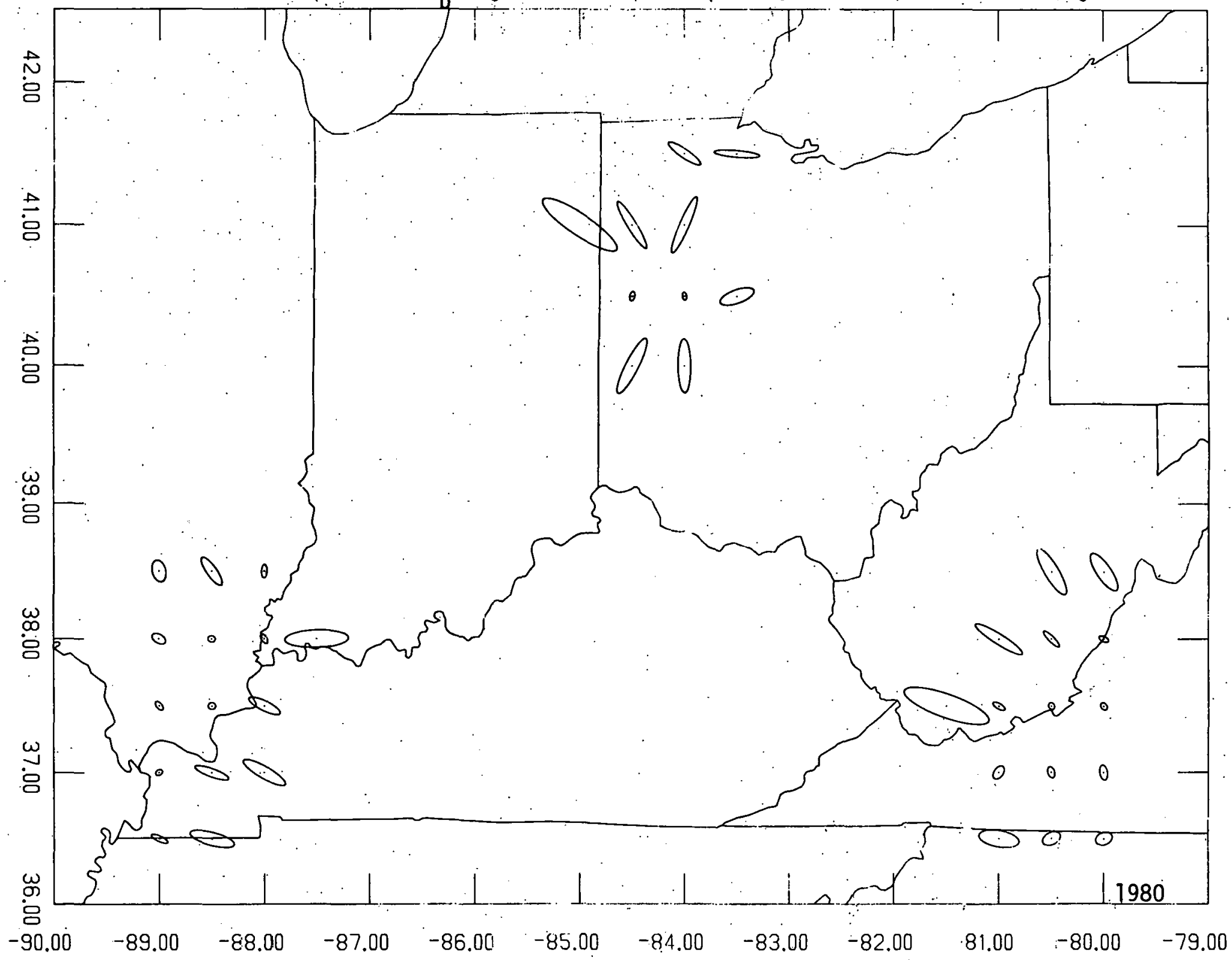


$m_{b} 2.0$ events (figure 14, Appendix IIh). These earthquakes are small enough that they cannot be located accurately except within the dimensions of high density arrays. This is clearly démonstrated in figure 14 where ellipses are drawn within the Wabash Valley Array, the Anna, Ohio Array and the West Virginia-Virginia border array. Location capability drops to zero very quickly outside the dimensions of the local network. 
Arnold, E. P. (1968),' Smoothing Travel Time Tables', Bull. Seis: Soc. Amer., Vol. 58, p. 1345.

Booker, A.J. (1964), Estimation of Network Capability, DATDA Rept. No. 98, January.

Evernden, J:F. (1967), 'Magnitude determination at regional and nearregional distances in the United States', Bull. Seis. Soc. Amer., Vol. 57, p. 591 .

Flinn, E. A. (1964),' Confidence regions and error determinations for seismic event location', S D Lab. Rept. No. 103, Teledyne Geotech, Alexandria, Va.

Geiger, L. (1910),' Herdbestimmung bei Erdbeben aus den Ankunftzeiten', K. Gesell. Wiss. Gott., Vol. 4, p. 331.

Herrin, E. et a1. (1968),' Special Number - 1968 Seismological Tables for P Phases', Bull. Seis. Soc. Amer., Vol. 58, p. 1193.

Richter, C. F. (1937), Elementary Seismology, W. H. Freeman and Co. Press, San Francisco, Calif., p. 342.

Wirth, M. H., R. Blandford, and H. Husted (1978), ' Estimation of Network Detection and Location Ability', DARPA Order No. 2551, Teledyne Geotech, Alexandria, Va. 


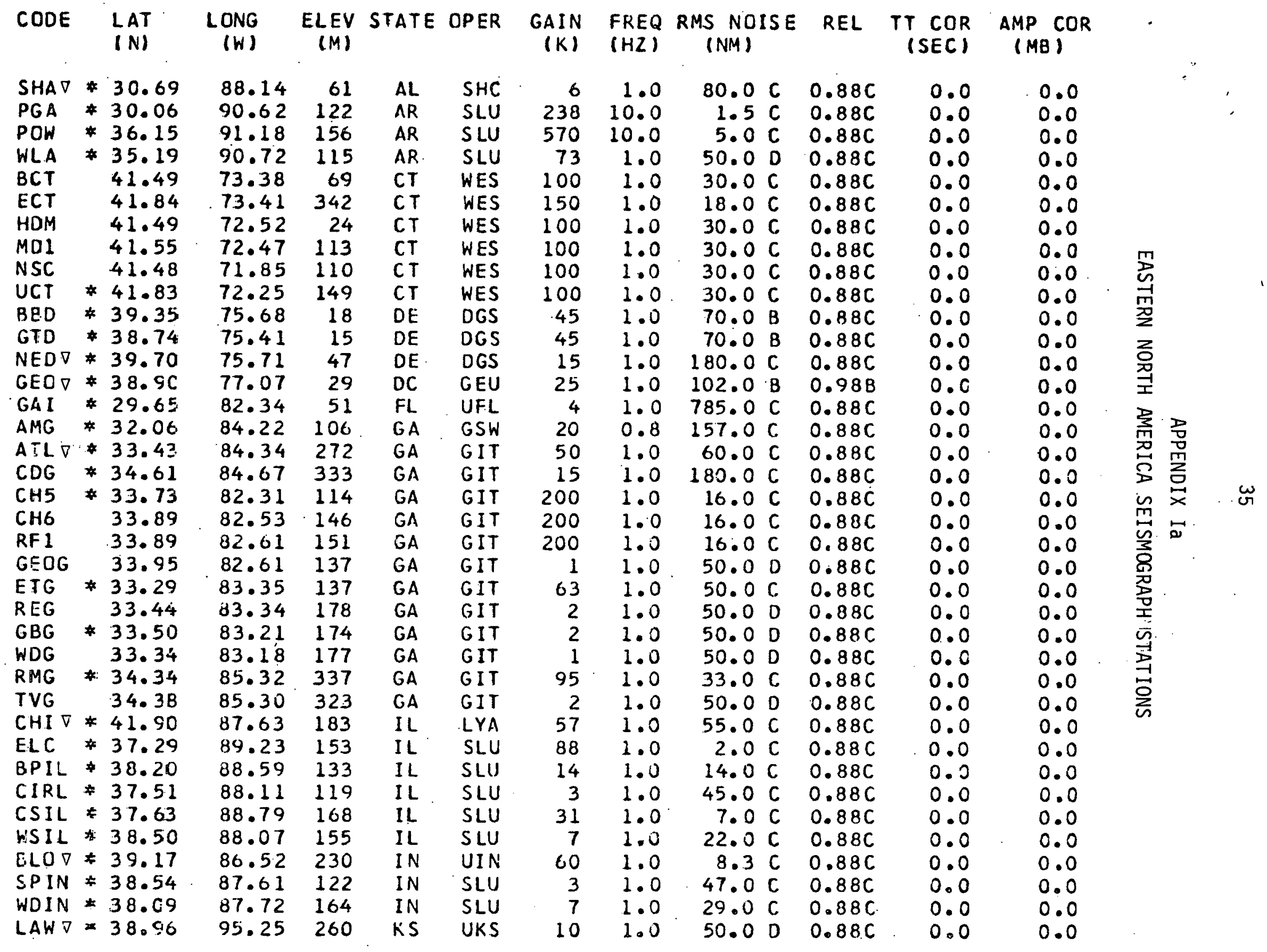




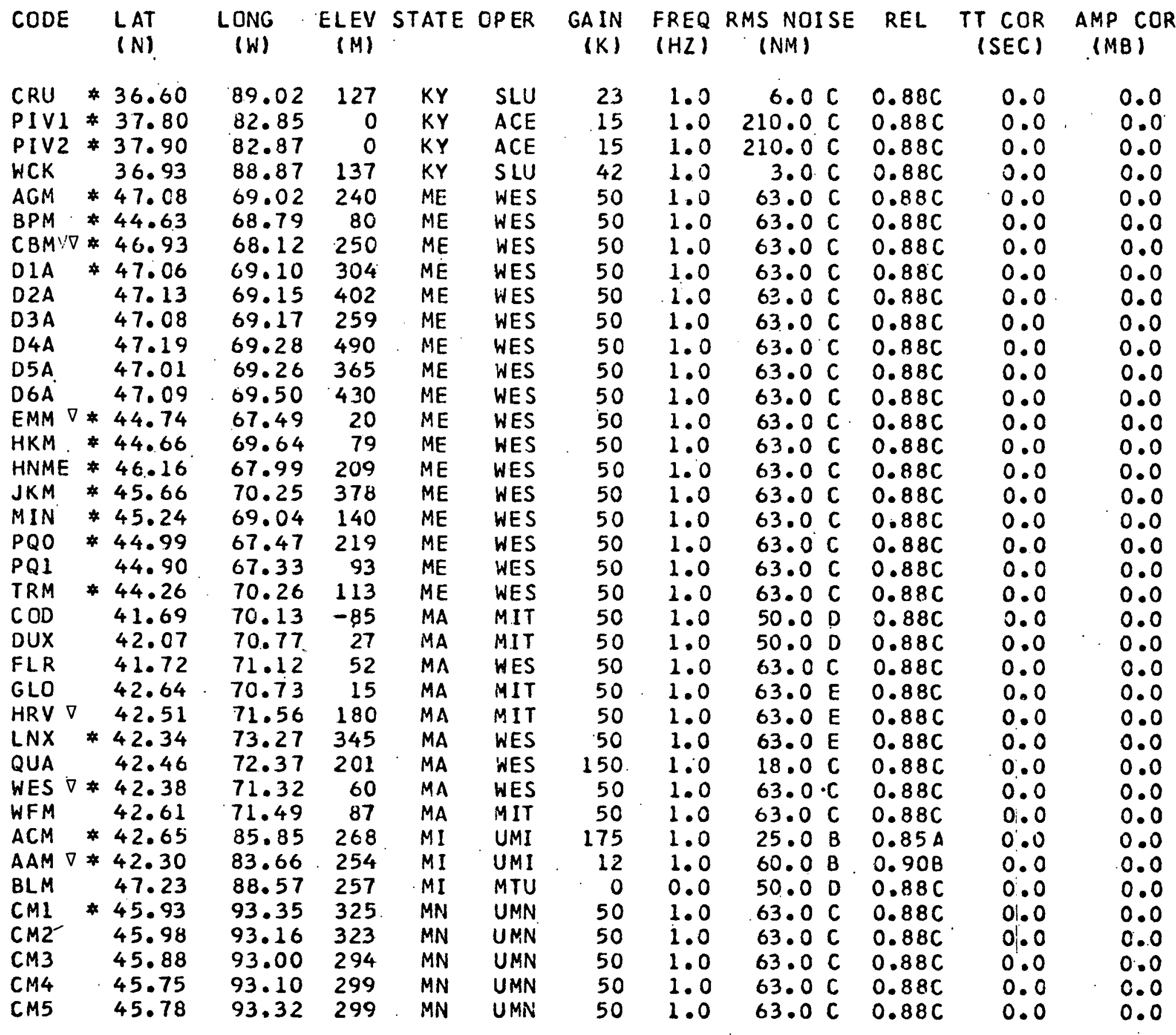




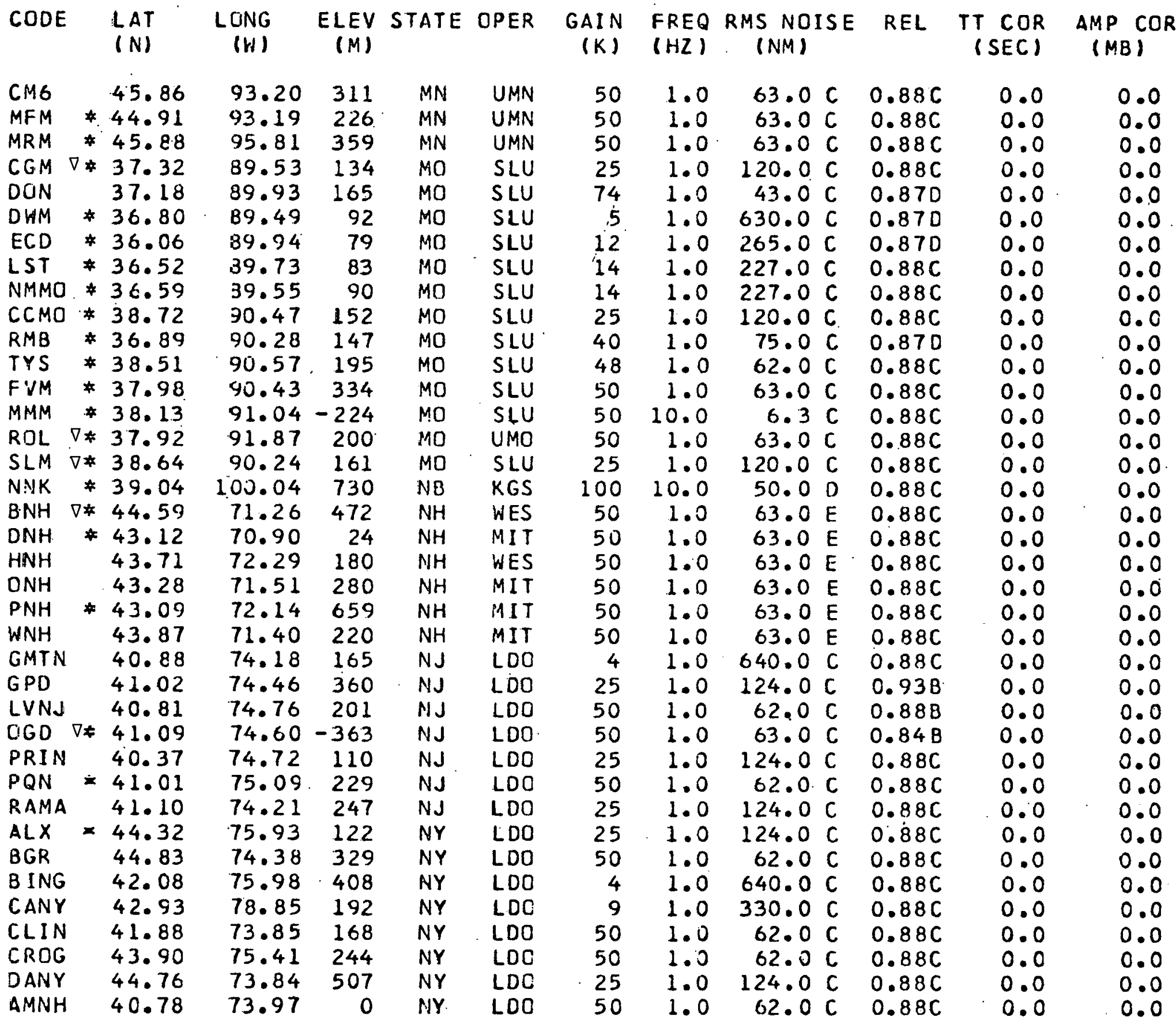




\begin{tabular}{|c|c|c|c|c|c|c|c|c|c|c|c|c|c|}
\hline :ODE & & $\begin{array}{l}\text { LAT } \\
\text { (N) }\end{array}$ & $\begin{array}{r}\text { LONG } \\
(W)\end{array}$ & $\begin{array}{r}E L E V \\
(M)\end{array}$ & STATE & DPER & $\begin{array}{r}\text { GAIN } \\
(K)\end{array}$ & $\begin{array}{l}\text { FREQ } \\
(H Z)\end{array}$ & $\begin{array}{r}\text { RMS N } \\
\text { (NM }\end{array}$ & & REL & $\begin{array}{r}T T \text { COR } \\
\text { (SEC) }\end{array}$ & $\begin{array}{c}\text { AMP COR } \\
(M B)\end{array}$ \\
\hline $\begin{array}{l}\text { MSNY } \\
\text { MEDY } \\
\text { PAL } \nabla \\
\text { LOO } \\
\text { PNY } \\
\text { PTN } \\
\text { SANY } \\
\text { TBR } \\
\text { WPR } \\
\text { WPNY } \\
\text { WVLY } \\
\text { WNY } \\
\text { WND } \\
\text { APH } \\
\text { CTR } \\
\text { CLY } \\
\text { EGN } \\
\text { DCN } \\
\text { UWL } \\
\text { ATT } \\
\text { DNY } \\
\text { DHN } \\
\text { BLM } \\
\text { DBM } \\
\text { DPL } \\
\text { GOB } \\
\text { IPS } \\
\text { OSB } \\
\text { SNP } \\
\text { SPS } \\
\text { SRM } \\
\text { STL } \\
\text { WGL } \\
\text { BNY } \nabla \\
\text { BUF } \nabla \\
\text { INY } \\
\text { ROC } \nabla \\
\text { TRY }\end{array}$ & $\begin{array}{l}* \\
\text { * } \\
\text { * } \\
\text { * } \\
\text { * }\end{array}$ & $\begin{array}{l}45.00 \\
43.18 \\
41.00 \\
41.00 \\
44.83 \\
44.57 \\
43.15 \\
41.14 \\
41.26 \\
41.80 \\
42.47 \\
44.39 \\
42.34 \\
43.84 \\
43.87 \\
43.85 \\
43.86 \\
43.88 \\
43.84 \\
42.84 \\
42.84 \\
42.83 \\
41.33 \\
41.29 \\
41.25 \\
41.33 \\
41.27 \\
41.36 \\
41.24 \\
41.30 \\
41.23 \\
41.19 \\
41.36 \\
42.09 \\
42.93 \\
42.44 \\
43.13 \\
42.73\end{array}$ & $\begin{array}{l}74.86 \\
78.39 \\
73.91 \\
73.91 \\
73.55 \\
74.98 \\
78.88 \\
74.22 \\
73.59 \\
73.97 \\
78.57 \\
73.86 \\
74.15 \\
74.50 \\
74.46 \\
74.45 \\
74.48 \\
74.53 \\
74.54 \\
78.19 \\
78.17 \\
78.19 \\
73.96 \\
73.98 \\
73.91 \\
73.92 \\
73.95 \\
73.92 \\
73.97 \\
73.89 \\
74.01 \\
74.00 \\
73.90 \\
75.97 \\
78.85 \\
76.48 \\
77.59 \\
73.67\end{array}$ & $\begin{array}{r}55 \\
187 \\
91 \\
91 \\
177 \\
238 \\
172 \\
261 \\
152 \\
76 \\
600 \\
598 \\
602 \\
564 \\
585 \\
579 \\
547 \\
701 \\
561 \\
470 \\
381 \\
491 \\
134 \\
27 \\
67 \\
150 \\
0 \\
212 \\
30 \\
168 \\
165 \\
125 \\
152 \\
291 \\
195 \\
238 \\
155 \\
118\end{array}$ & $\begin{array}{l}\text { NY } \\
\text { NY } \\
\text { NY } \\
\text { NY } \\
\text { NY } \\
\text { NY } \\
\text { NY } \\
\text { NY } \\
\text { NY } \\
\text { NY } \\
\text { NY } \\
\text { NY } \\
\text { NY } \\
\text { NY } \\
\text { NY } \\
\text { NY } \\
\text { NY } \\
\text { NY } \\
\text { NY } \\
\text { NY } \\
\text { NY } \\
\text { NY } \\
\text { NY } \\
\text { NY } \\
\text { NY } \\
\text { NY } \\
\text { NY } \\
\text { NY } \\
\text { NY } \\
\text { NY } \\
\text { NY } \\
\text { NY } \\
\text { NY } \\
\text { NY } \\
\text { NY } \\
\text { NY } \\
\text { NY } \\
\text { NY }\end{array}$ & 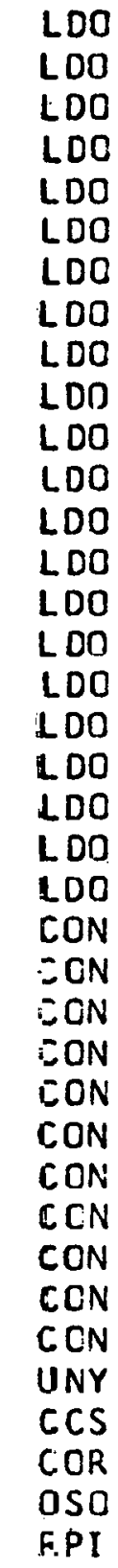 & $\begin{array}{r}0 \\
9 \\
100 \\
9 \\
9 \\
50 \\
25 \\
25 \\
50 \\
50 \\
25 \\
50 \\
50 \\
101 \\
101 \\
50 \\
25 \\
202 \\
50 \\
25 \\
13 \\
50 \\
0 \\
0 \\
0 \\
0 \\
0 \\
50 \\
0 \\
0 \\
0 \\
0 \\
0 \\
75 \\
0 \\
85 \\
0 \\
3\end{array}$ & $\begin{array}{l}0.0 \\
1.0 \\
1.0 \\
1.0 \\
1.0 \\
1.0 \\
1.0 \\
1.0 \\
1.0 \\
1.0 \\
1.0 \\
1.0 \\
1.0 \\
1.0 \\
1.0 \\
1.0 \\
1.0 \\
1.0 \\
1.0 \\
1.0 \\
1.0 \\
1.0 \\
0.0 \\
0.0 \\
0.0 \\
0.0 \\
0.0 \\
1.0 \\
0.0 \\
0.0 \\
0.0 \\
0.0 \\
0.0 \\
0.0 \\
0.0 \\
1.0 \\
0.0 \\
22.0\end{array}$ & $\begin{array}{r}50.0 \\
330.0 \\
31.0 \\
330.0 \\
330.0 \\
62.0 \\
124.0 \\
124.0 \\
62.0 \\
62.0 \\
124.0 \\
62.0 \\
62.0 \\
30.0 \\
30.0 \\
62.0 \\
124.0 \\
16.0 \\
62.0 \\
124.0 \\
234.0 \\
63.0 \\
50.0 \\
50.0 \\
50.0 \\
50.0 \\
50.0 \\
63.0 \\
50.0 \\
50.0 \\
10.0 \\
50.0 \\
50.0 \\
6.0 \\
50.0 \\
5.09 \\
53.0 \\
63.0\end{array}$ & $\begin{array}{l}D \\
C \\
C \\
C \\
C \\
C \\
C \\
C \\
C \\
C \\
C \\
C \\
C \\
C \\
C \\
C \\
C \\
C \\
C \\
C \\
C \\
E \\
D \\
D \\
D \\
D \\
D \\
E \\
D \\
D \\
E \\
D \\
D \\
C \\
D \\
C \\
C \\
E\end{array}$ & $\begin{array}{l}0.88 C \\
0.88 C \\
0.88 C \\
0.88 C \\
0.88 C \\
0.88 C \\
0.88 C \\
0.92 B \\
0.88 C \\
0.88 C \\
0.88 C \\
0.93 B \\
0.88 C \\
0.88 C \\
0.88 C \\
0.88 C \\
0.88 C \\
0.88 C \\
0.88 C \\
0.88 C \\
0.88 C \\
0.88 C \\
0.88 C \\
0.88 C \\
0.88 C \\
0.88 C \\
0.88 C \\
0.88 C \\
0.88 C \\
0.88 C \\
0.88 C \\
0.88 C \\
0.88 C \\
0.88 C \\
0.88 C \\
0.88 C \\
0.88 C \\
0.88 C\end{array}$ & $\begin{array}{l}0.0 \\
0.0 \\
0.0 \\
0.0 \\
0.0 \\
0.0 \\
0.0 \\
0.0 \\
0.0 \\
0.0 \\
0.0 \\
0.0 \\
0.0 \\
0.0 \\
0.0 \\
0.0 \\
0.0 \\
0.0 \\
0.0 \\
0.0 \\
0.0 \\
0.0 \\
0.0 \\
0.0 \\
0.0 \\
0.0 \\
0.0 \\
0.0 \\
0.0 \\
0.0 \\
0.0 \\
0.0 \\
0.0 \\
0.0 \\
0.0 \\
0.0 \\
0.0 \\
0.0\end{array}$ & $\begin{array}{l}0.0 \\
0.0 \\
0.0 \\
0.0 \\
0.0 \\
0.0 \\
0.0 \\
0.0 \\
0.0 \\
0.0 \\
0.0 \\
0.0 \\
0.0 \\
0.0 \\
0.0 \\
0.0 \\
0.0 \\
0.0 \\
0.0 \\
0.0 \\
0.0 \\
0.0 \\
0.0 \\
0.0 \\
0.0 \\
0.0 \\
0.0 \\
0.0 \\
0.0 \\
0.0 \\
0.0 \\
0.0 \\
0.0 \\
0.0 \\
0.0 \\
0.0 \\
0.0 \\
0.0\end{array}$ \\
\hline
\end{tabular}




\begin{tabular}{|c|c|c|c|c|c|c|c|c|c|c|c|c|}
\hline 10 & & $\begin{array}{l}\text { LAT } \\
\text { (N) }\end{array}$ & $\begin{array}{l}\text { ONG } \\
(W)\end{array}$ & $\begin{array}{l}\text { LEV } \\
\text { (M) }\end{array}$ & TATE & OPER & $\begin{array}{l}\text { FIN } \\
(\mathrm{K})\end{array}$ & $\begin{array}{l}\text { FREQ } \\
(H Z)\end{array}$ & $\begin{array}{l}\text { RMS NOI SE } \\
\text { (NM) }\end{array}$ & REL. & $\begin{array}{c}\text { TT COR } \\
(S E C)\end{array}$ & $\begin{array}{l}A M P C O \\
(M B)\end{array}$ \\
\hline $\begin{array}{l}\text { CEH } \\
\text { RNC } \\
\text { ANI } \\
\text { AN3 } \\
\text { AN4 } \\
\text { AN7 } \\
\text { AN8 } \\
\text { AN9 } \\
\text { AN10 } \\
\text { ANI1 } \\
\text { ANI2 } \\
\text { CLE } \\
\text { UTO } \\
\text { BGO } \\
\text { MLF } \\
\text { OLO } \\
\text { SCP } \\
\text { BVR } \\
\text { ERP } \\
\text { MVL } \\
\text { KTZ } \\
\text { BCS } \\
\text { CCS } \\
\text { CHF } \\
\text { HBF } \\
\text { JSC } \\
\text { LHS } \\
\text { MGS } \\
\text { MTT } \\
\text { NHS } \\
\text { OSB } \\
\text { PBS } \\
\text { PRM } \\
\text { SGS } \\
\text { SVS } \\
\text { VSC } \\
\text { BOW } \\
\text { COW }\end{array}$ & 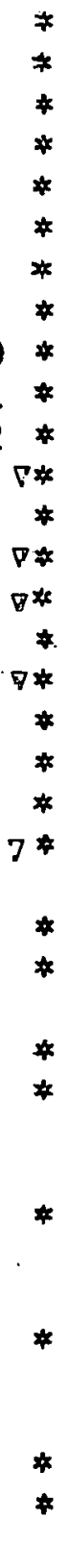 & $\begin{array}{l}35.89 \\
35.63 \\
40.48 \\
40.55 \\
40.22 \\
40.82 \\
40.24 \\
40.71 \\
40.47 \\
40.55 \\
40.92 \\
41.49 \\
41.66 \\
41.38 \\
39.14 \\
36.46 \\
40.79 \\
40.70 \\
42.13 \\
39.99 \\
40.51 \\
32.98 \\
32.82 \\
34.02 \\
32.93 \\
34.28 \\
34.48 \\
32.90 \\
33.75 \\
33.07 \\
33.55 \\
33.28 \\
34.08 \\
33.19 \\
32.97 \\
32.88 \\
33.37 \\
33.38\end{array}$ & $\begin{array}{l}79.09 \\
78.98 \\
84.13 \\
83.81 \\
83.81 \\
83.86 \\
84.29 \\
84.50 \\
84.47 \\
84.68 \\
84.18 \\
81.53 \\
83.57 \\
83.66 \\
84.28 \\
95.71 \\
77.87 \\
80.33 \\
79.98 \\
76.37 \\
75.78 \\
80.07 \\
80.26 \\
82.59 \\
80.38 \\
81.26 \\
80.81 \\
80.14 \\
81.64 \\
79.76 \\
80.84 \\
80.26 \\
82.36 \\
80.51 \\
80.25 \\
81.05 \\
80.64 \\
80.70\end{array}$ & $\begin{array}{r}152 \\
85 \\
306 \\
326 \\
346 \\
281 \\
302 \\
255 \\
275 \\
273 \\
226 \\
328 \\
0 \\
212 \\
238 \\
196 \\
352 \\
0 \\
0 \\
91 \\
148 \\
12 \\
9 \\
152 \\
10 \\
120 \\
120 \\
9 \\
182 \\
10 \\
91 \\
25 \\
254 \\
25 \\
33 \\
30 \\
35 \\
33\end{array}$ & $\begin{array}{l}N C \\
N C \\
O H \\
O H \\
O H \\
O H \\
O H \\
O H \\
O H \\
O H \\
O H \\
O H \\
O H \\
O H \\
O H \\
O H \\
O K \\
P A \\
P A \\
P A \\
P A \\
P A \\
S C \\
S C \\
S C \\
S C \\
S C \\
S C \\
S C \\
S C \\
S C \\
S C \\
S C \\
S C \\
S C \\
S C \\
S C \\
S C \\
S C\end{array}$ & $\begin{array}{l}\text { UNC } \\
\text { CPL } \\
\text { UMI } \\
\text { UMI } \\
\text { UNI } \\
\text { UMI } \\
\text { UMI } \\
\text { UMI } \\
\text { UMI } \\
\text { UMI } \\
\text { UMI } \\
\text { JCU } \\
\text { UTO } \\
\text { BGU } \\
\text { XAU } \\
\text { UOK } \\
P S U \\
P S U \\
P S U \\
\text { MSC } \\
\text { KSC } \\
\text { SGS } \\
\text { SGS } \\
\text { SGS } \\
\text { SGS } \\
\text { SGS } \\
\text { SGS } \\
\text { SGS } \\
\text { SGS } \\
\text { SGS } \\
\text { SGS } \\
\text { SGS } \\
\text { SGS } \\
\text { SGS } \\
\text { SGS } \\
\text { SGS } \\
\text { SGS } \\
\text { SGS }\end{array}$ & $\begin{array}{r}2 \\
134 \\
175 \\
175 \\
175 \\
175 \\
175 \\
175 \\
175 \\
175 \\
175 \\
9 \\
140 \\
30 \\
0 \\
140 \\
50 \\
50 \\
50 \\
0 \\
4 \\
26 \\
54 \\
26 \\
124 \\
240 \\
124 \\
13 \\
62 \\
62 \\
31 \\
31 \\
240 \\
124 \\
54 \\
31 \\
31 \\
0\end{array}$ & $\begin{array}{l}1.0 \\
1.0 \\
2.0 \\
2.0 \\
2.0 \\
2.0 \\
2.0 \\
2.0 \\
2.0 \\
2.0 \\
2.0 \\
1.0 \\
1.0 \\
1.0 \\
0.0 \\
10.0 \\
1.0 \\
1.0 \\
1.0 \\
0.0 \\
1.0 \\
2.0 \\
2.0 \\
2.0 \\
2.0 \\
2.0 \\
2.0 \\
2.0 \\
2.0 \\
2.0 \\
2.0 \\
2.0 \\
2.0 \\
2.0 \\
2.0 \\
2.0 \\
2.0 \\
0.0\end{array}$ & $\begin{array}{r}250.0 \mathrm{C} \\
3.7 \mathrm{C} \\
6.0 \mathrm{~B} \\
5.0 \mathrm{~A} \\
6.0 \mathrm{~B} \\
7.0 \mathrm{~B} \\
5.0 \mathrm{~A} \\
5.0 \mathrm{~B} \\
6.0 \mathrm{~B} \\
5.0 \mathrm{~B} \\
5.0 \mathrm{~B} \\
600.0 \mathrm{C} \\
22.0 \mathrm{C} \\
17.0 \mathrm{C} \\
50.0 \mathrm{D} \\
50.0 \mathrm{C} \\
42.0 \mathrm{~B} \\
63.0 \mathrm{C} \\
63.0 \mathrm{C} \\
50.0 \mathrm{C} \\
785.0 \mathrm{C} \\
60.0 \mathrm{C} \\
29.0 \mathrm{C} \\
60.0 \mathrm{C} \\
12.4 \mathrm{C} \\
6.2 \mathrm{C} \\
12.4 \mathrm{C} \\
112.0 \mathrm{C} \\
25.0 \mathrm{C} \\
25.0 \mathrm{C} \\
50.0 \mathrm{C} \\
50.0 \mathrm{C} \\
6.2 \mathrm{C} \\
12.4 \mathrm{C} \\
50.0 \mathrm{C} \\
50.0 \mathrm{C} \\
50.0 \mathrm{O} \\
50.0 \mathrm{C}\end{array}$ & $\begin{array}{l}0.88 C \\
0.88 C \\
0.94 A \\
0.85 A \\
0.80 A \\
0.70 A \\
0.74 A \\
0.84 A \\
0.77 A \\
0.86 A \\
0.85 A \\
0.88 C \\
0.88 C \\
0.88 C \\
0.88 C \\
0.88 C \\
0.88 C \\
0.88 C \\
0.88 C \\
0.88 C \\
0.88 C \\
0.88 C \\
0.88 C \\
0.88 C \\
0.88 C \\
0.88 C \\
0.88 C \\
0.88 C \\
0.88 C \\
0.88 C \\
0.88 C \\
0.88 C \\
0.88 C \\
0.88 C \\
0.88 C \\
0.88 C \\
0.88 C \\
0.88 C\end{array}$ & $\begin{array}{l}0.0 \\
0.0 \\
0.0 \\
0.0 \\
0.0 \\
0.0 \\
0.0 \\
0.0 \\
0.0 \\
0.0 \\
0.0 \\
0.0 \\
0.0 \\
0.0 \\
0.0 \\
0.0 \\
0.0 \\
0.0 \\
0.0 \\
0.0 \\
0.0 \\
0.0 \\
0.0 \\
0.0 \\
0.0 \\
0.0 \\
0.0 \\
0.0 \\
0.0 \\
0.0 \\
0.0 \\
0.0 \\
0.0 \\
0.0 \\
0.0\end{array}$ & $\begin{array}{l}0.0 \\
0.0 \\
0.0 \\
0.0 \\
0.0 \\
0.0 \\
0.0 \\
0.0 \\
0.0 \\
0.0 \\
0.0 \\
0.0 \\
0.0 \\
0.0 \\
0.0 \\
0.0 \\
0.0 \\
0.0 \\
0.0 \\
0.0 \\
0.0 \\
0.0 \\
0.0 \\
0.0 \\
0.0 \\
0.0 \\
0.0 \\
0.0\end{array}$ \\
\hline
\end{tabular}




\begin{tabular}{|c|c|c|c|c|c|c|c|c|c|c|c|c|c|}
\hline CODE & & $\begin{array}{l}\text { LAT } \\
(N)\end{array}$ & $\begin{array}{l}\text { LUNG } \\
(W)\end{array}$ & $\begin{array}{l}\text { ELEV } \\
(M)\end{array}$ & TATE & CPER & $\begin{array}{c}\text { GAIN } \\
(K)\end{array}$ & $\begin{array}{l}\text { FREQ } \\
(H Z)\end{array}$ & $\begin{array}{r}\text { RMS NO } \\
(N M)\end{array}$ & $S E$ & REL & $\begin{array}{l}T T \text { COR } \\
\text { (SEC) }\end{array}$ & $\begin{array}{l}\text { AMP COR } \\
(M B)\end{array}$ \\
\hline $\begin{array}{l}\text { WMS } \\
\text { ROW } \\
\text { ZIN } \\
\text { HRO } 1 \\
\text { MRO2 } \\
\text { MRO3 } \\
\text { MRO4 } \\
\text { MRO5 } \\
\text { MRO6 } \\
\text { MRO7 } \\
\text { MRO8 } \\
\text { MRO9 } \\
\text { SMT } \\
\text { SRPD } \\
\text { SRPN } \\
\text { SRPW } \\
\text { GRT } \\
\text { NKT } \\
\text { CKG } \\
\text { MPH } \\
\text { TKL } \\
\text { ORT } \nabla \\
\text { MET } \\
\text { LGAR } \\
\text { PGM } \\
\text { DLY } \\
\text { SFTN } \\
\text { CPO } 7 \\
\text { DAL } \nabla \\
\text { COV } \\
\text { FLET } \\
\text { MARL } \\
\text { MDV } \nabla \\
\text { MGVT } \\
\text { MPVT } \\
\text { BVT } \\
\text { DVT } \\
\text { IVT }\end{array}$ & $\begin{array}{l}* \\
* \\
* \\
* \\
* \\
*\end{array}$ & $\begin{array}{l}33.44 \\
33.37 \\
33.11 \\
34.33 \\
34.19 \\
34.35 \\
34.43 \\
34.43 \\
34.35 \\
34.37 \\
34.41 \\
34.30 \\
34.93 \\
33.15 \\
33.33 \\
33.20 \\
36.26 \\
35.85 \\
35.63 \\
35.12 \\
35.66 \\
35.91 \\
35.12 \\
34.65 \\
34.46 \\
35.50 \\
35.36 \\
35.60 \\
32.85 \\
44.58 \\
44.72 \\
42.38 \\
44.00 \\
44.91 \\
44.34 \\
43.35 \\
44.96 \\
43.52\end{array}$ & $\begin{array}{l}80.74 \\
80.79 \\
80.16 \\
81.30 \\
81.23 \\
81.46 \\
81.46 \\
81.22 \\
81.20 \\
81.32 \\
81.41 \\
81.40 \\
82.97 \\
81.71 \\
81.59 \\
81.58 \\
89.42 \\
89.54 \\
89.84 \\
89.93 \\
83.77 \\
84.30 \\
89.93 \\
90.66 \\
90.11 \\
91.47 \\
90.02 \\
85.57 \\
96.78 \\
73.15 \\
72.95 \\
72.80 \\
73.18 \\
72.63 \\
72.58 \\
72.59 \\
72.17 \\
73.05\end{array}$ & $\begin{array}{r}34 \\
35 \\
30 \\
131 \\
84 \\
125 \\
125 \\
145 \\
137 \\
134 \\
112 \\
122 \\
498 \\
31 \\
95 \\
77 \\
137 \\
153 \\
129 \\
94 \\
350 \\
370 \\
93 \\
0 \\
105 \\
236 \\
23 \\
574 \\
187 \\
85 \\
366 \\
580 \\
134 \\
262 \\
240 \\
300 \\
370 \\
295\end{array}$ & $\begin{array}{l}S C \\
S C \\
S C \\
S C \\
S C \\
S C \\
S C \\
S C \\
S C \\
S C \\
S C \\
S C \\
S C \\
S C \\
S C \\
S C \\
T N \\
T N \\
T N \\
T N \\
T N \\
T N \\
T N \\
T N \\
T N \\
T N \\
T N \\
T N \\
T X \\
V T \\
V T \\
V T \\
V T \\
V T \\
V T \\
V T \\
V T \\
V T\end{array}$ & $\begin{array}{l}\text { SGS } \\
\text { SGS } \\
\text { SGS } \\
\text { SCG } \\
\text { SCG } \\
\text { SCG } \\
\text { SCG } \\
\text { SCG } \\
\text { SCG } \\
\text { SCG } \\
\text { SCG } \\
\text { SCG } \\
U S C \\
\text { DUP } \\
\text { DUP } \\
\text { DUP } \\
\text { SLU } \\
\text { SLU } \\
\text { SLU } \\
\text { NSU } \\
\text { TEC } \\
\text { LCC } \\
\text { MSU } \\
\text { MSU } \\
\text { HSU } \\
M S U \\
\text { MSU } \\
\text { TGS } \\
\text { SMU } \\
\text { LOO } \\
\text { LDO } \\
\text { LDC } \\
\text { LDO } \\
\text { LDO } \\
\text { LDO } \\
\text { WES } \\
\text { WES } \\
\text { WES }\end{array}$ & $\begin{array}{r}0 \\
0 \\
0 \\
7 \\
7 \\
7 \\
7 \\
7 \\
7 \\
7 \\
7 \\
7 \\
0 \\
9 \\
9 \\
25 \\
23 \\
21 \\
25 \\
25 \\
15 \\
70 \\
1 \\
73 \\
73 \\
504 \\
73 \\
400 \\
12 \\
50 \\
101 \\
50 \\
101 \\
50 \\
50 \\
50 \\
50 \\
50\end{array}$ & $\begin{array}{l}0.0 \\
0.0 \\
0.0 \\
1.0 \\
1.0 \\
1.0 \\
1.0 \\
1.0 \\
1.0 \\
1.0 \\
1.0 \\
1.0 \\
0.0 \\
5.0 \\
5.0 \\
5.0 \\
1.0 \\
1.0 \\
1.0 \\
1.0 \\
1.0 \\
1.0 \\
30.0 \\
1.0 \\
1.0 \\
1.0 \\
1.0 \\
1.0 \\
1.0 \\
1.0 \\
1.0 \\
1.0 \\
1.0 \\
1.0 \\
1.0 \\
1.0 \\
1.0 \\
1.0\end{array}$ & $\begin{array}{r}50.0 \\
50.0 \\
50.0 \\
415.0 \\
415.0 \\
415.0 \\
415.0 \\
415.0 \\
415.0 \\
415.0 \\
415.0 \\
415.0 \\
50.0 \\
250.0 \\
250.0 \\
100.0 \\
7.0 \\
6.0 \\
5.0 \\
5.0 \\
210.0 \\
44.0 \\
50.0 \\
50.0 \\
50.0 \\
50.0 \\
50.0 \\
9.7 \\
40.0 \\
63.0 \\
30.0 \\
62.0 \\
30.0 \\
61.0 \\
62.0 \\
63.0 \\
63.0 \\
63.0\end{array}$ & $\begin{array}{l}D \\
D \\
E \\
C \\
E \\
E \\
E \\
E \\
E \\
E \\
E \\
E \\
D \\
C \\
C \\
C \\
B \\
B \\
B \\
B \\
C \\
C \\
D \\
D \\
D \\
D \\
D \\
C \\
C \\
C \\
C \\
C \\
C \\
C \\
C \\
E \\
E \\
E\end{array}$ & $\begin{array}{l}0.88 C \\
0.88 C \\
0.88 C \\
0.88 C \\
0.88 C \\
0.88 C \\
0.88 C \\
0.88 C \\
0.88 C \\
0.88 C \\
0.88 C \\
0.88 C \\
0.88 C \\
0.88 C \\
0.88 C \\
0.88 C \\
0.88 C \\
0.88 C \\
0.88 C \\
0.88 C \\
0.88 C \\
0.88 C \\
0.88 C \\
0.88 C \\
0.88 C \\
0.88 C \\
0.88 C \\
0.88 C \\
0.88 C \\
0.88 C \\
0.88 C \\
0.88 C \\
0.88 C \\
0.88 C \\
0.88 C \\
0.88 C \\
0.88 C \\
0.88 C\end{array}$ & $\begin{array}{l}0.0 \\
0.0 \\
0.0 \\
0.0 \\
0.0 \\
0.0 \\
0.0 \\
0.0 \\
0.0 \\
0.0 \\
0.0 \\
0.0 \\
0.0 \\
0.0 \\
0.0 \\
0.0 \\
0.0 \\
0.0 \\
0.0 \\
0.0 \\
0.0 \\
0.0 \\
0.0 \\
0.0 \\
0.0 \\
0.0 \\
0.0 \\
0.0 \\
0.0 \\
0.0 \\
0.0 \\
0.0 \\
0.0 \\
0.0 \\
0.0 \\
0.0 \\
0.0 \\
0.0\end{array}$ & $\begin{array}{l}0.0 \\
0.0 \\
0.0 \\
0.0 \\
0.0 \\
0.0 \\
0.0 \\
0.0 \\
0.0 \\
0.0 \\
0.0 \\
0.0 \\
0.0 \\
0.0 \\
0.0 \\
0.0 \\
0.0 \\
0.0 \\
0.0 \\
0.0 \\
0.0 \\
0.0 \\
0.0 \\
0.0 \\
0.0 \\
0.0 \\
0.0 \\
0.0 \\
0.0 \\
0.0 \\
0.0 \\
0.0 \\
0.0 \\
0.0 \\
0.0 \\
0.0 \\
0.0 \\
0.0\end{array}$ \\
\hline
\end{tabular}




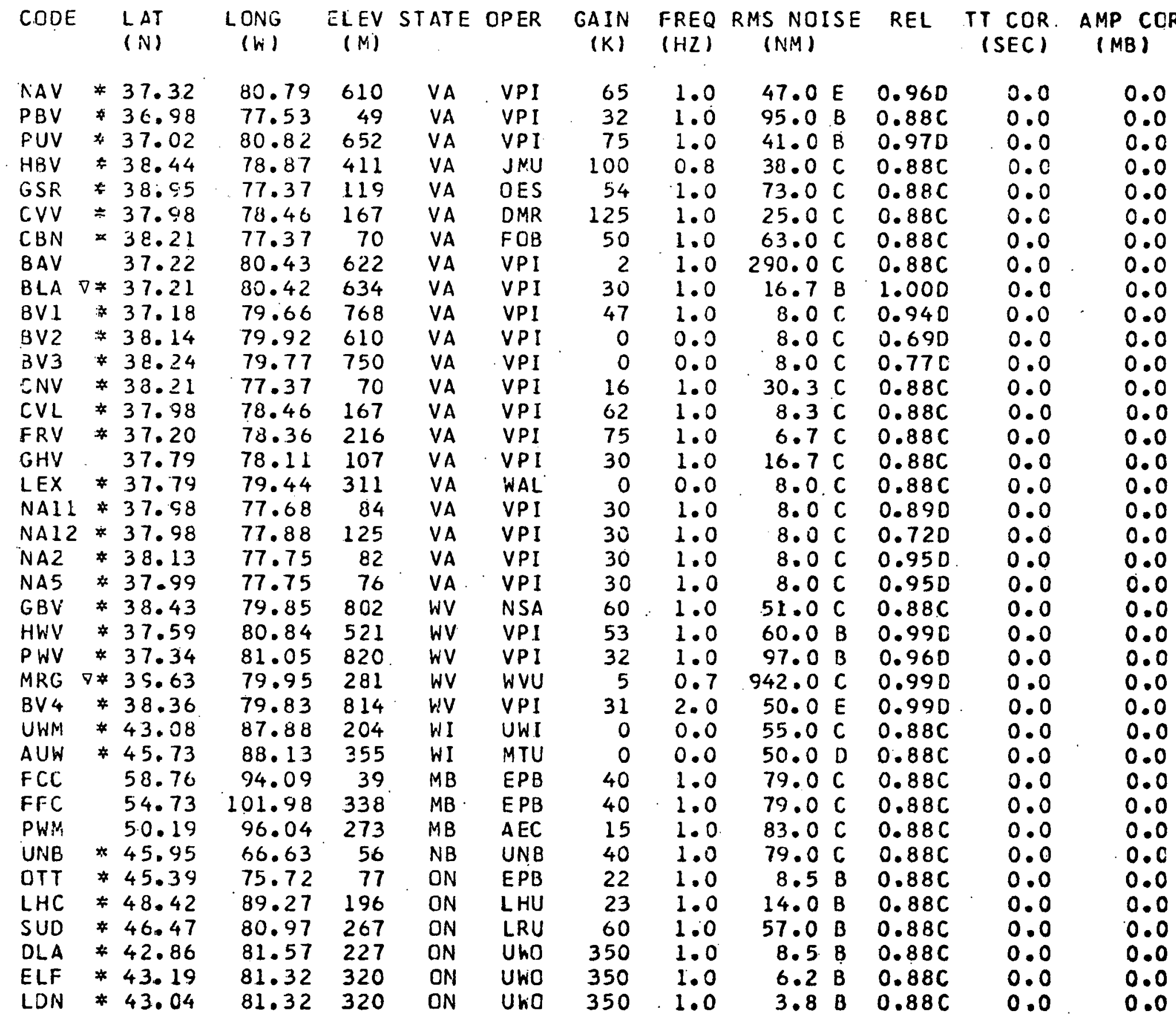




\begin{tabular}{|c|c|c|c|c|c|c|c|c|c|c|c|c|}
\hline CODE & & $\begin{array}{l}\text { LAT } \\
(N)\end{array}$ & $\begin{array}{l}\text { LONG } \\
(W)\end{array}$ & $\begin{array}{r}E L E V \\
(M)\end{array}$ & STATE & OPER & $\begin{array}{r}\text { GAIN } \\
(K)\end{array}$ & $\begin{array}{l}\text { FREQ } \\
(H Z)\end{array}$ & $\begin{array}{l}\text { RMS NOISE } \\
\text { (NM) }\end{array}$ & REL & $\begin{array}{l}\text { TT COR } \\
(S E C)\end{array}$ & $\begin{array}{l}\text { AMP COR } \\
(M B)\end{array}$ \\
\hline $\begin{array}{l}\text { CFO } \\
\text { CHQ } \\
\text { LGQ } \\
\text { LMQ } \\
\text { MNT } \\
\text { PBO } \\
\text { POC } \\
\text { QCQ } \\
\text { SCH } \\
\text { SIC }\end{array}$ & * & $\begin{array}{l}45.47 \\
46.89 \\
53.69 \\
47.55 \\
45.50 \\
55.28 \\
47.36 \\
46.78 \\
54.82 \\
5 c .17\end{array}$ & $\begin{array}{l}76.23 \\
71.30 \\
77.73 \\
70.33 \\
73.62 \\
77.74 \\
70.04 \\
71.28 \\
60.78 \\
66.74\end{array}$ & $\begin{array}{r}70 \\
145 \\
190 \\
419 \\
112 \\
20 \\
61 \\
91 \\
540 \\
283\end{array}$ & $\begin{array}{l}O N \\
Q B \\
Q B \\
Q B \\
Q B \\
Q B \\
O B \\
Q B \\
\text { QB } \\
\text { QB }\end{array}$ & $\begin{array}{l}\text { OHC } \\
\text { LUC } \\
\text { JBC } \\
\text { COQ } \\
\text { JBF } \\
\text { LUC } \\
\text { LUC } \\
\text { LUC } \\
\text { MGU } \\
\text { ICC }\end{array}$ & $\begin{array}{l}15 \\
40 \\
98 \\
20 \\
60 \\
40 \\
25 \\
9 \\
40 \\
70\end{array}$ & $\begin{array}{l}1.0 \\
1.0 \\
1.0 \\
1.0 \\
1.0 \\
1.0 \\
1.0 \\
1.0 \\
1.0 \\
1.0\end{array}$ & 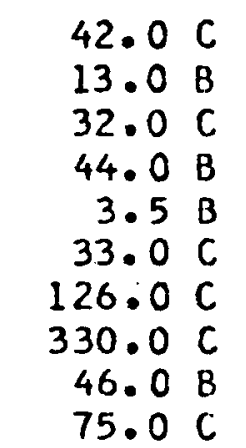 & $\begin{array}{l}0.88 \mathrm{C} \\
0.88 \mathrm{C} \\
0.88 \mathrm{C} \\
0.88 \mathrm{C} \\
0.88 \mathrm{C} \\
0.88 \mathrm{C} \\
0.88 \mathrm{C} \\
0.88 \mathrm{C} \\
0.88 \mathrm{C} \\
0.88 \mathrm{C}\end{array}$ & $\begin{array}{l}0.0 \\
0.0 \\
0.0 \\
0.0 \\
0.0 \\
0.0\end{array}$ & $\begin{array}{l}0.0 \\
0.0\end{array}$ \\
\hline
\end{tabular}




$\begin{array}{ll}\text { ACE } & \text { Army Corps. of Engineers } \\ \text { AEC } & \text { Atomic Energy of Canada Ltd. } \\ \text { BGU } & \text { Bowiing Green University } \\ \text { CCS } & \text { Canisius College Seismic Observatory } \\ \text { CON } & \text { Consolidated Edison of New York } \\ \text { COQ } & \text { Charlevoix Observatory of Quebec } \\ \text { COR } & \text { Cornell University } \\ \text { CPL } & \text { Carolina Power \& Light Company } \\ \text { DGS } & \text { Delaware Geologic Survey } \\ \text { DMR } & \text { Divisian of Minerals, Virginia } \\ \text { DUP } & \text { Dupont, Savannah River Laboratory } \\ \text { EPB } & \text { Earth and Physics Branch of Canada } \\ \text { FOB } & \text { Fredericksburg Observatory } \\ \text { GEU } & \text { Georgetown University } \\ \text { GIT } & \text { Georgia Institute of Technology } \\ \text { GSW } & \text { Georgia Southwest College } \\ \text { IOC } & \text { Iron Cre Corporation of Canada } \\ \text { JBC } & \text { James Bay Corporation of Canada } \\ \text { JBF } & \text { Jean-de-Breuf College, Canada } \\ & \end{array}$

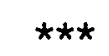




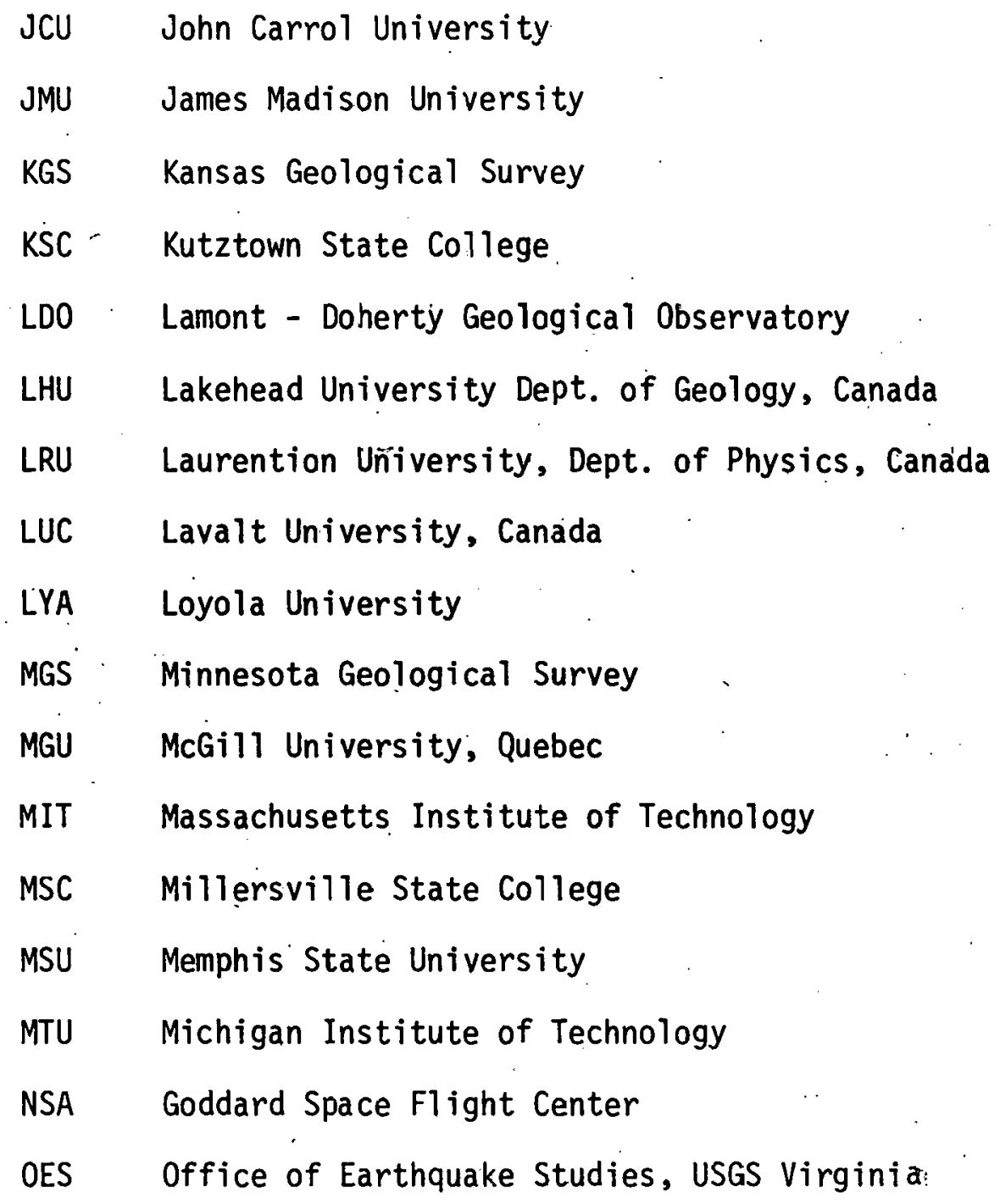




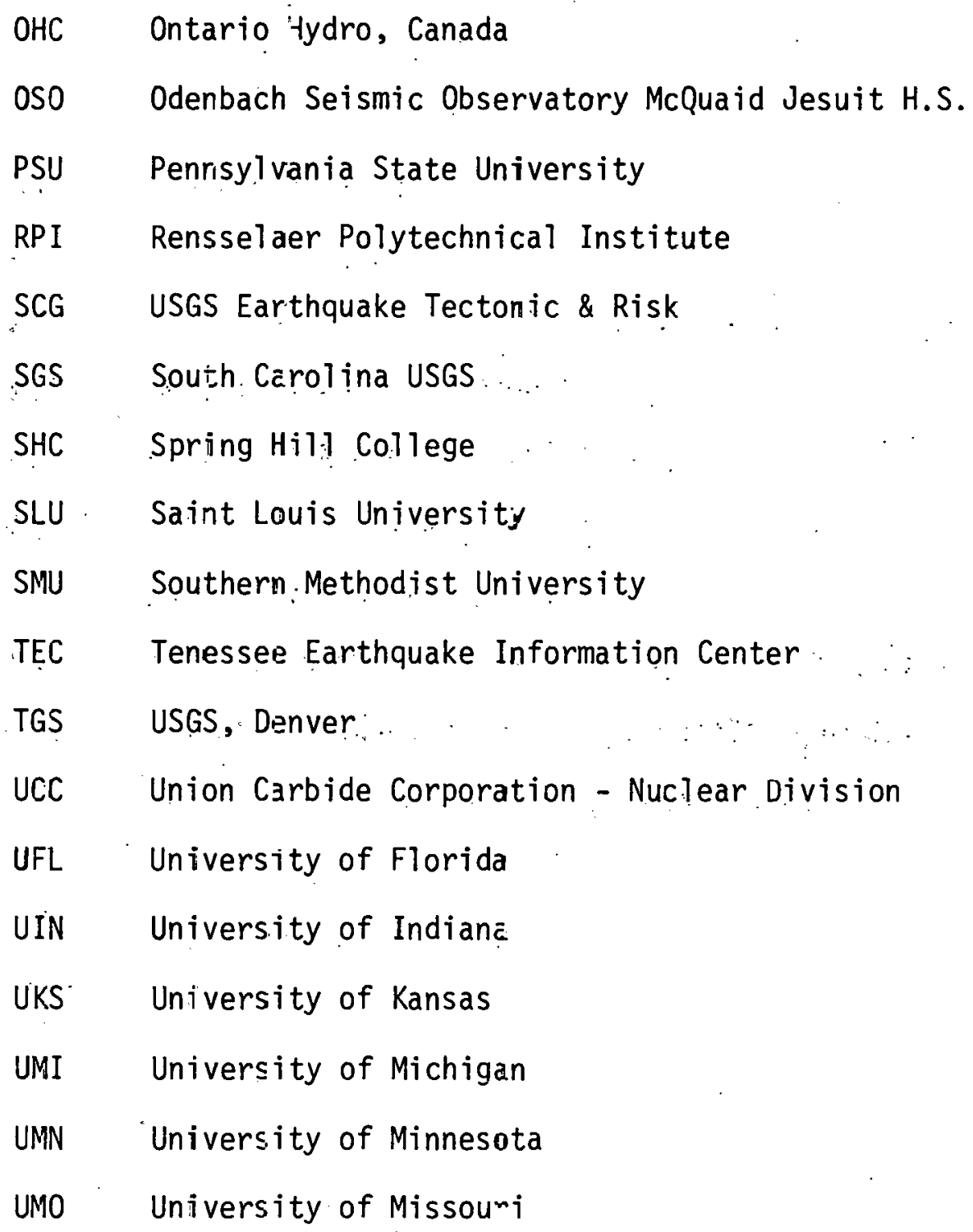




$\begin{array}{ll}\text { UNB } & \text { University of New Brunswick, Canada } \\ \text { UNC } & \text { University of North Carolina } \\ \text { UNY } & \text { State University of New York at Binghamton } \\ \text { UOK } & \text { University of Oklahoma } \\ \text { USC } & \text { University of South Carolina } \\ \text { UTO } & \text { University of Toledo } \\ \text { UWI } & \text { University of Wisconson at Milwaukee } \\ \text { UWO } & \text { University of Western Ontario, Canada } \\ \text { VPI } & \text { Virginia Polytechnical Institute } \\ \text { WAL } & \text { Washington \& Lee University } \\ \text { WCL } & \text { Woodward - Clyde Consulting Company } \\ \text { WES } & \text { Weston Observatory } \\ \text { WVU } & \text { West Virginia University } \\ \text { XAU } & \text { Xavier University }\end{array}$

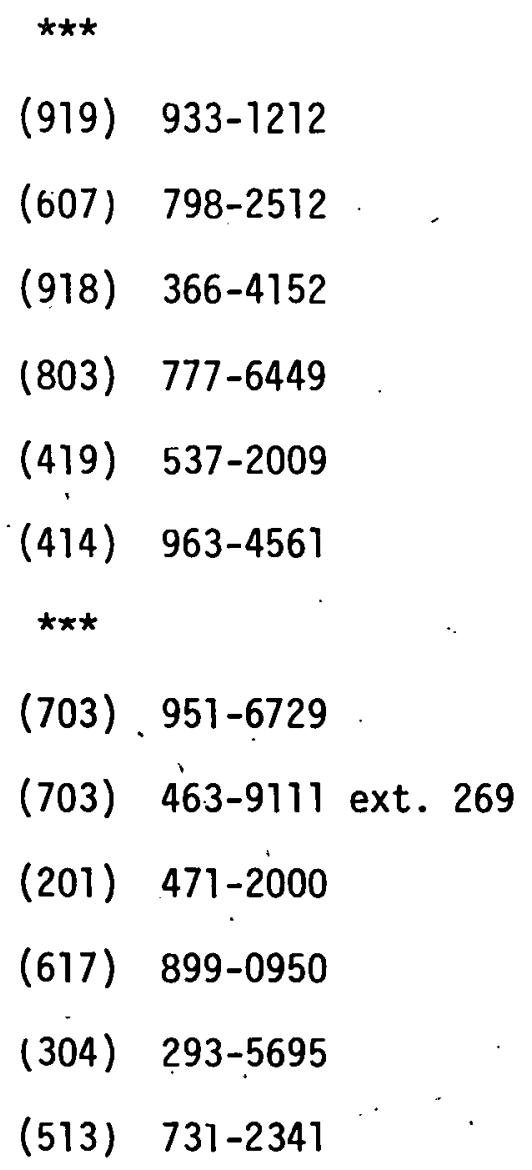


APPENDIX IC

CRITERIA FOR ASSIGNING NOISE AND RELIABILITY ESTIMATES FOR SEISMOGRAPH STATIONS

NOISE (Average rms background noise characteristics including both natural and cultural-sources)

A - Noise value determined by FFT of several characteristic digitized seismograms for the station

B - Noise measured on several months of records by University of Michigan Seismological Observatory staff

C - Noise estimated by assigning a peak to peak noise level of 1 millimeter on the records and reducing to the appropriate nanometer ground noise using. the instrumental gain

D - Noise default to an average background level of 50 nanometers

E - Noise assigned as a local array average

$F \quad$ - Noise determined by local operator

RELIABILITY $\left(P_{j}\right)$

A - Percentage of useful records determined by averaging hourly estimations over several months

B - Percentage of number of days the station is operational over several months

C - Percentage assigned by averaging all available reliability estimates provided by criteria $A, B$, and $D$

D - Reliability estimate provided by local operator

* - Station used in network performance evaluations 1980

$\nabla \quad$ - Station used in network performance evaluations 1970 
NETWJEK SUMMAKY- ERROR ELLIPSES

(MAGNITUDES EANGE FRUM, 5.00 TO 5.00 IN STEPS OP 0.0 )

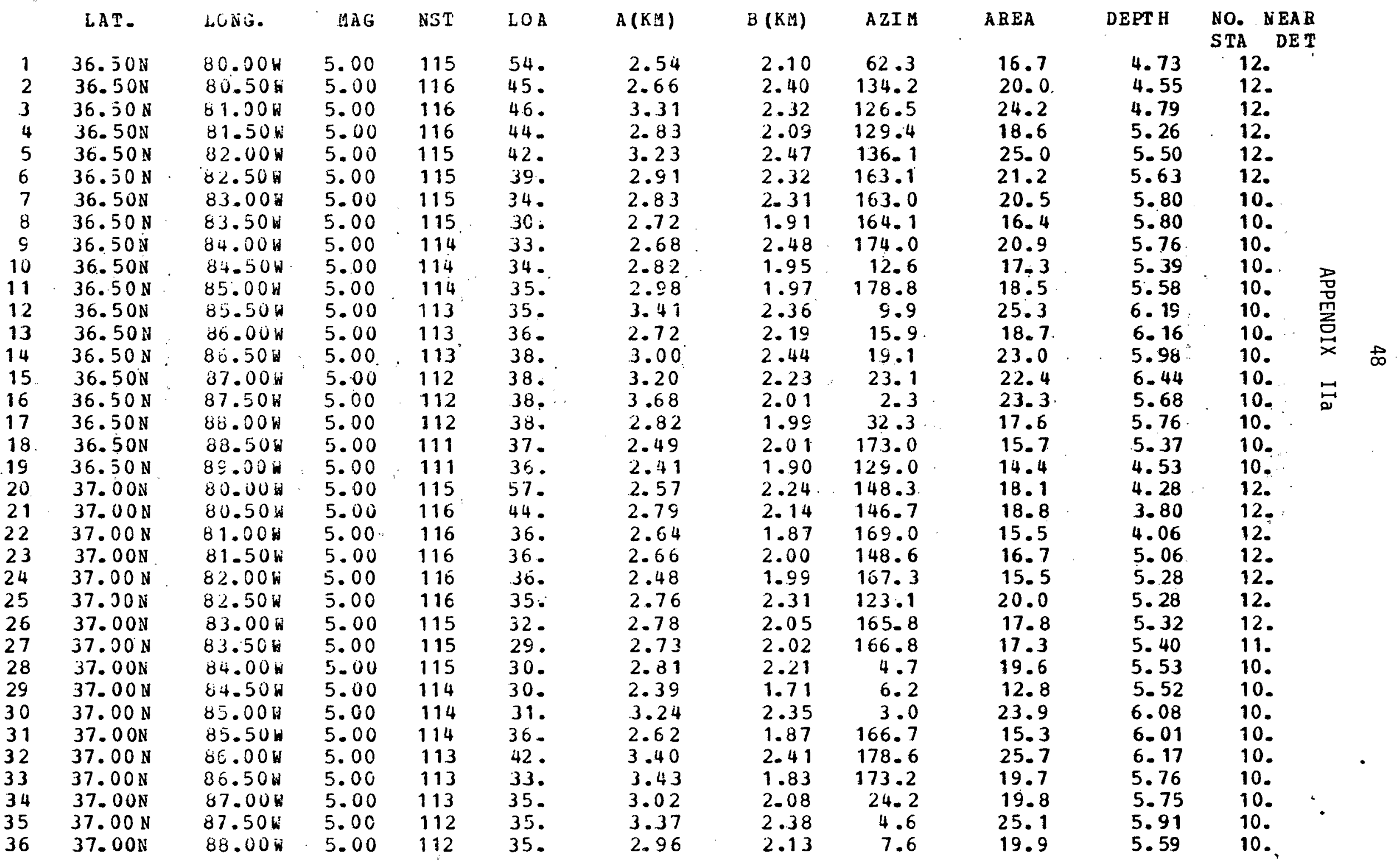




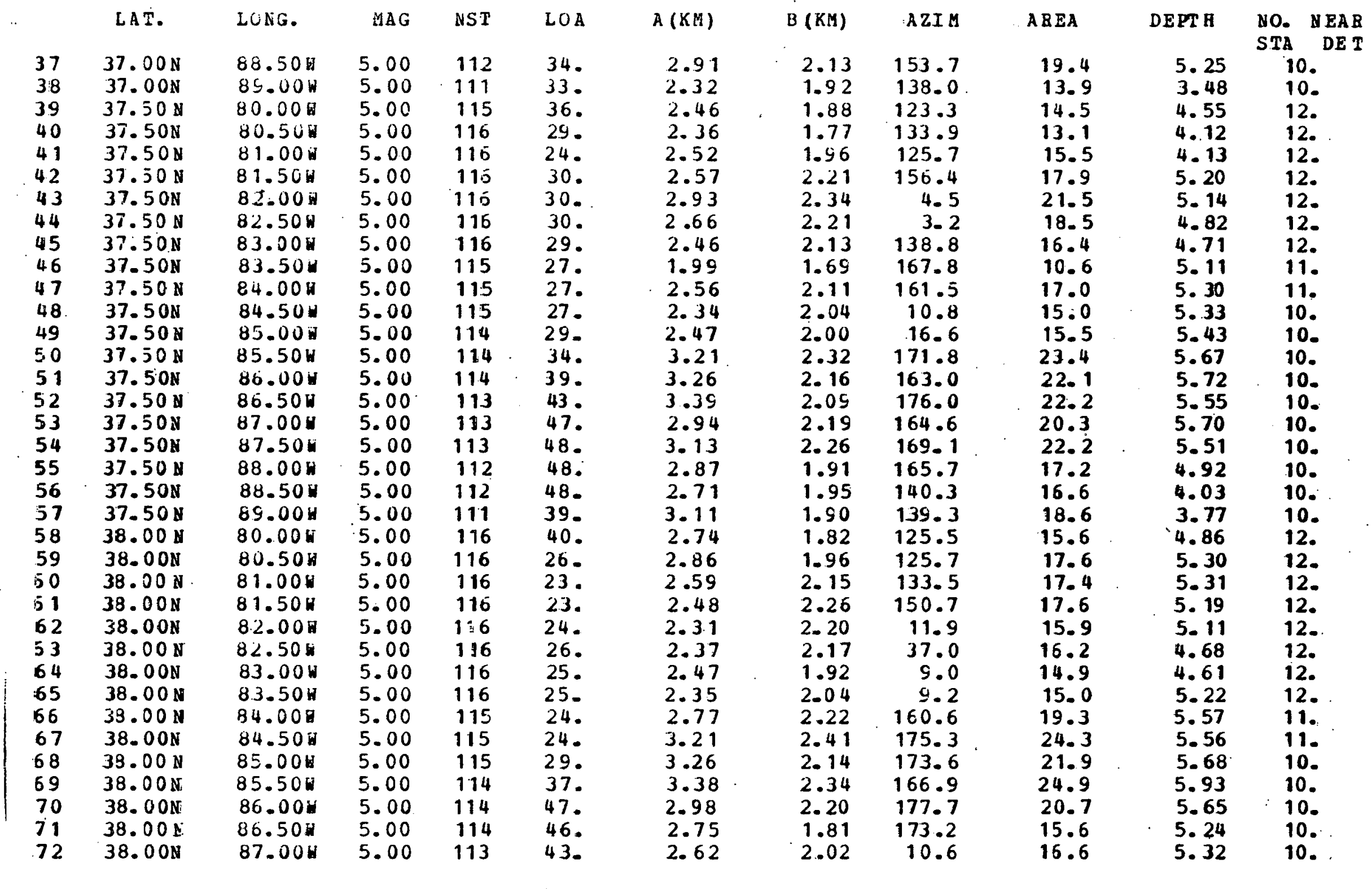




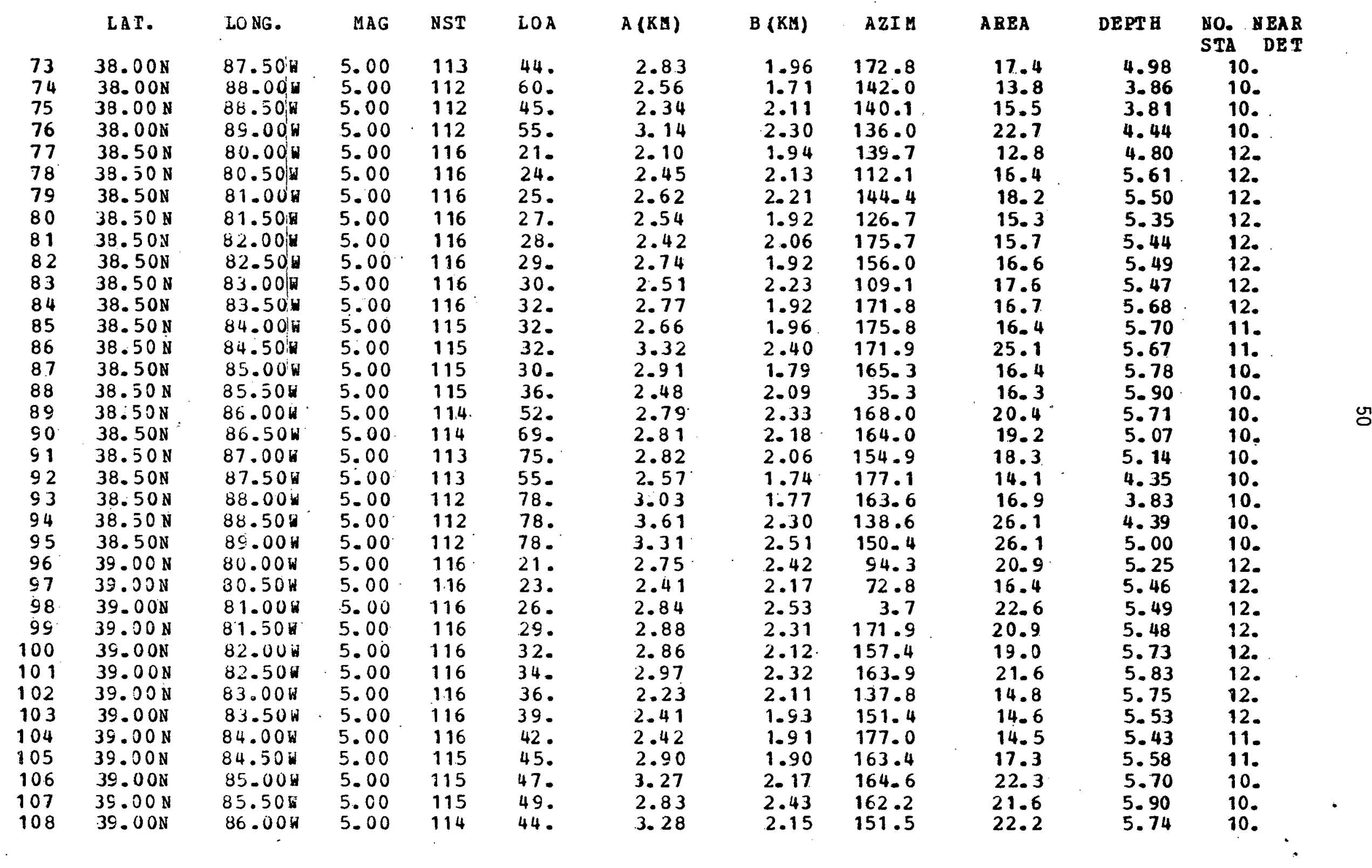




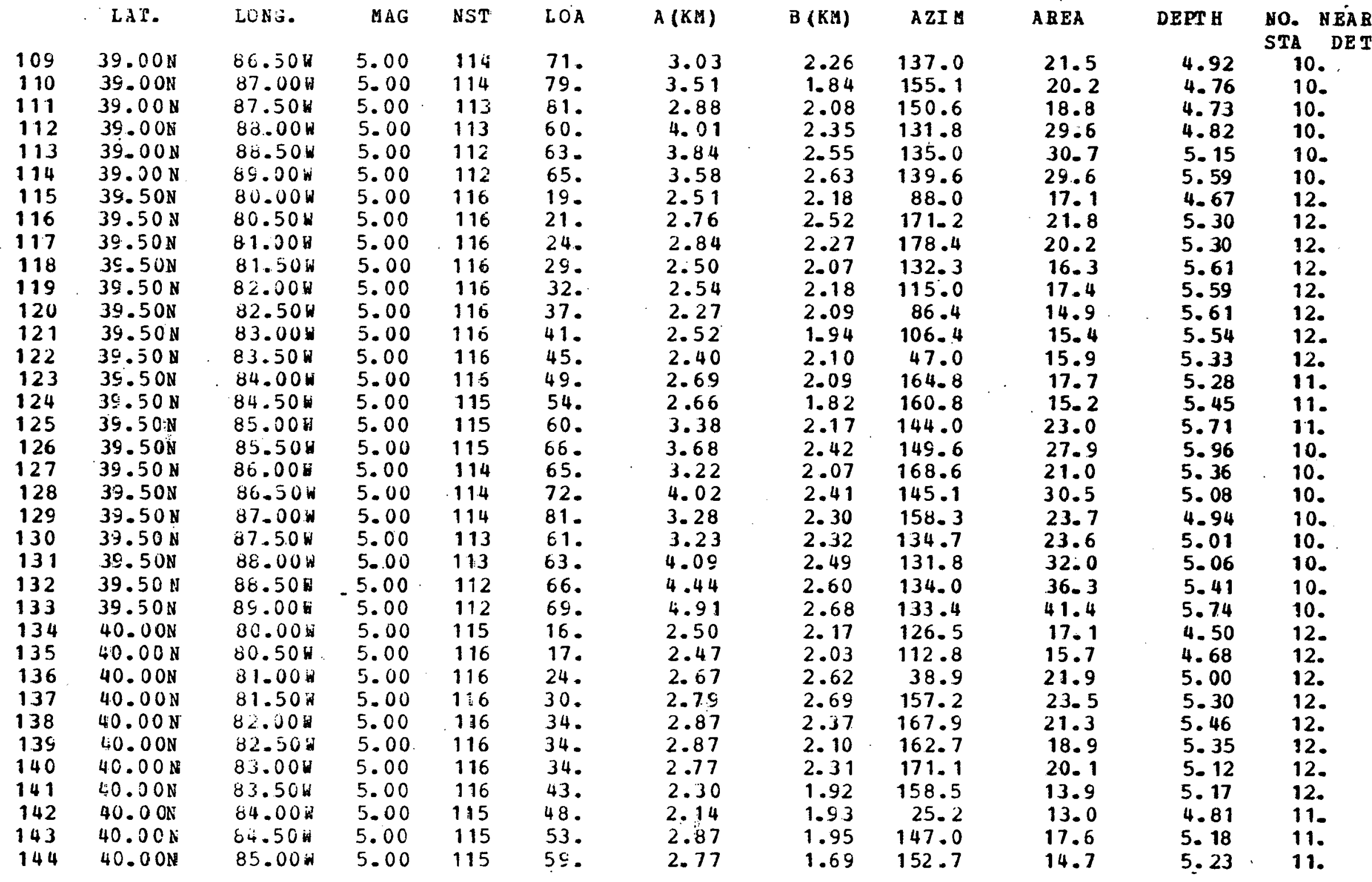




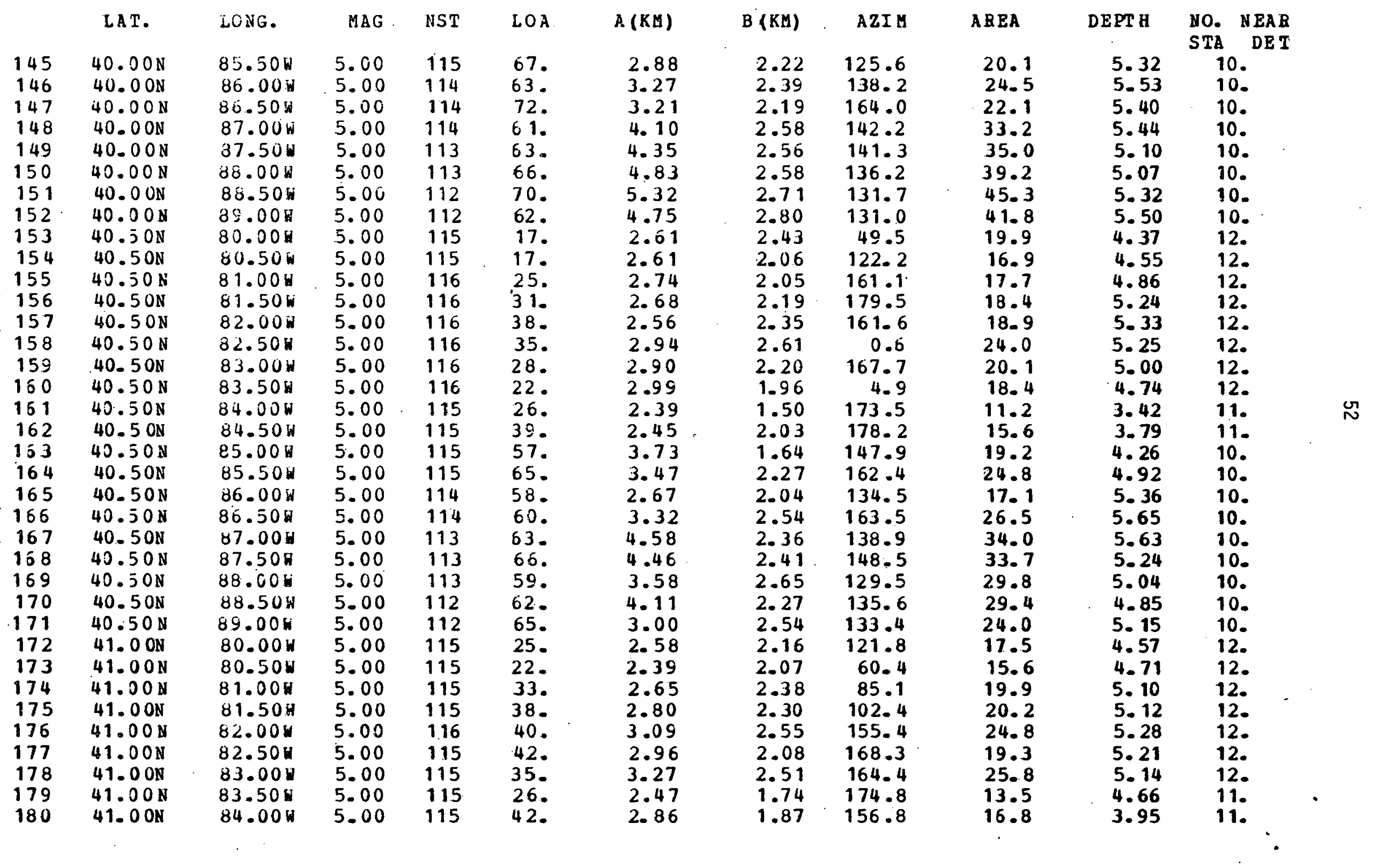




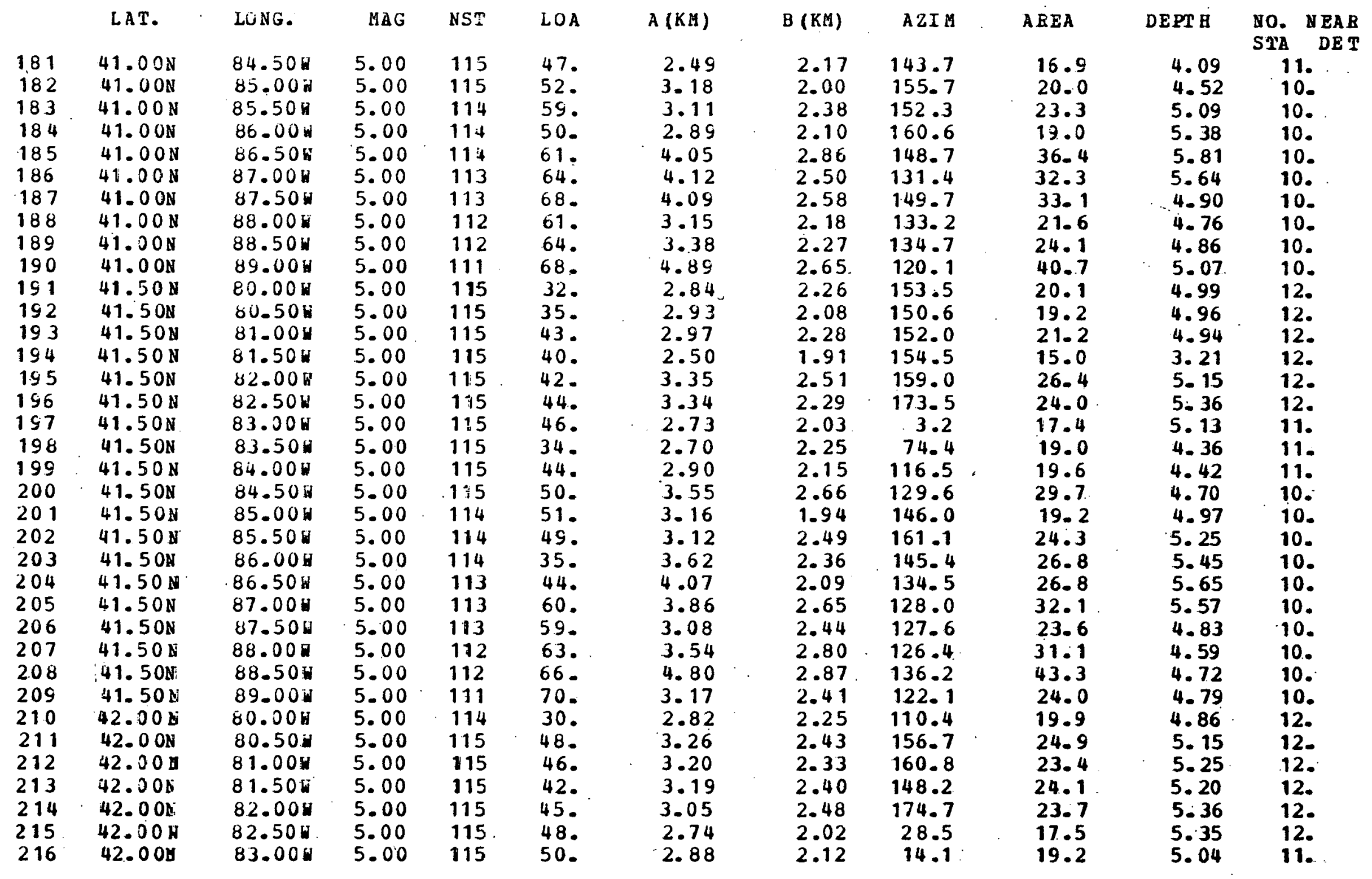




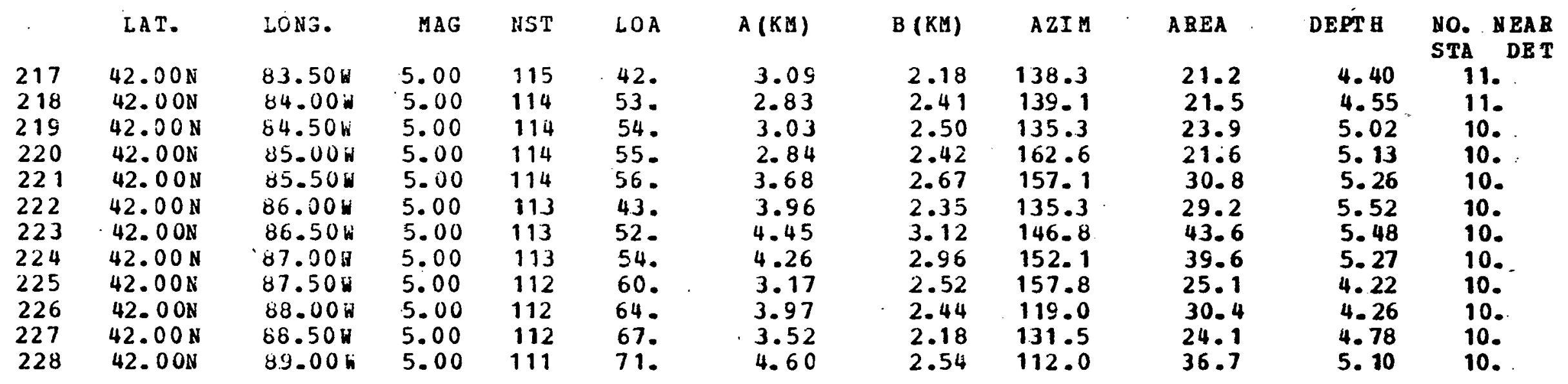


NETHORK SUHMARY - EBBOR ELLIPSES

(UAGNITUDES RANGE FROM, 4.50 TO 4.50 IN STEPS OF 0.0 )

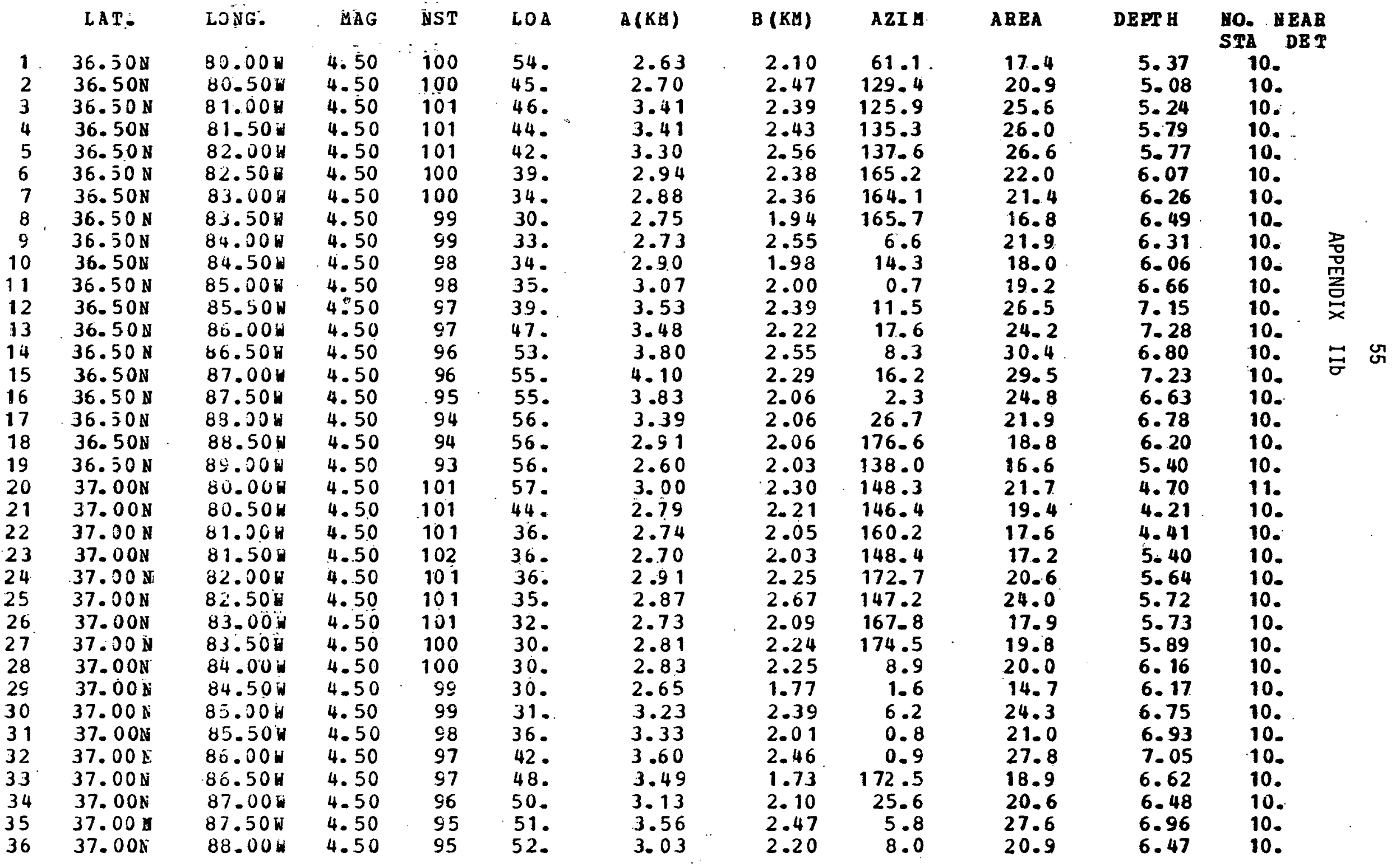




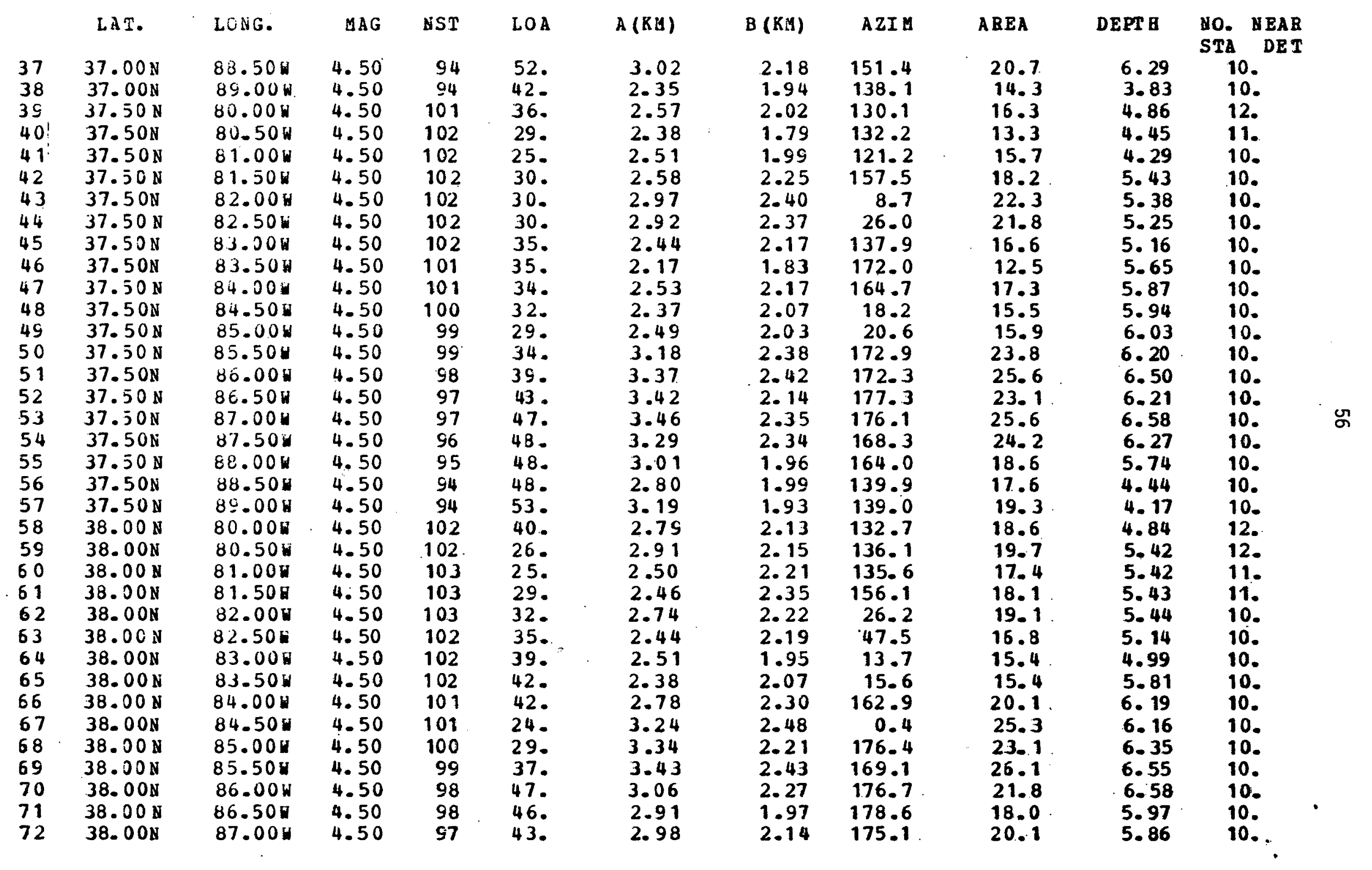




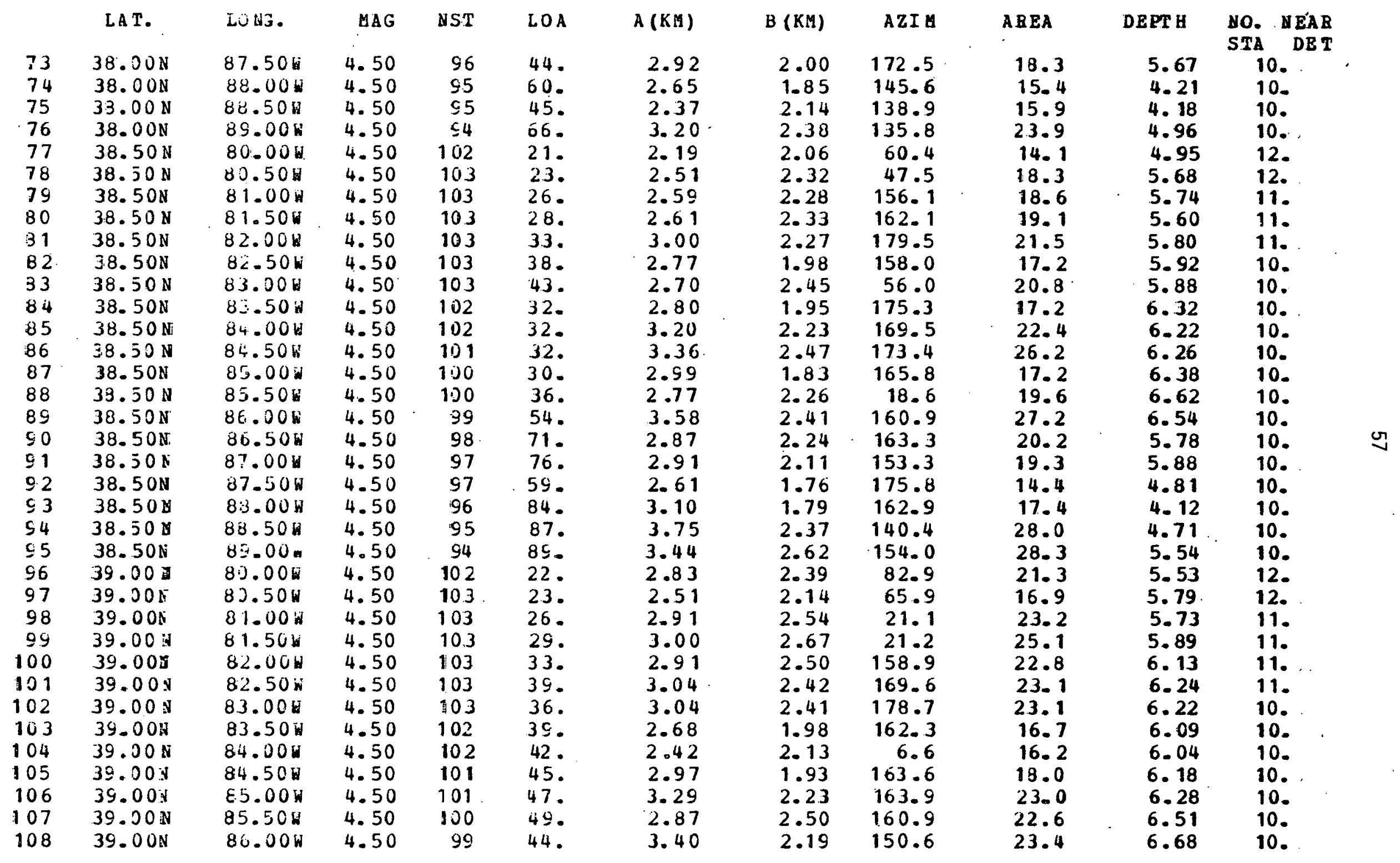




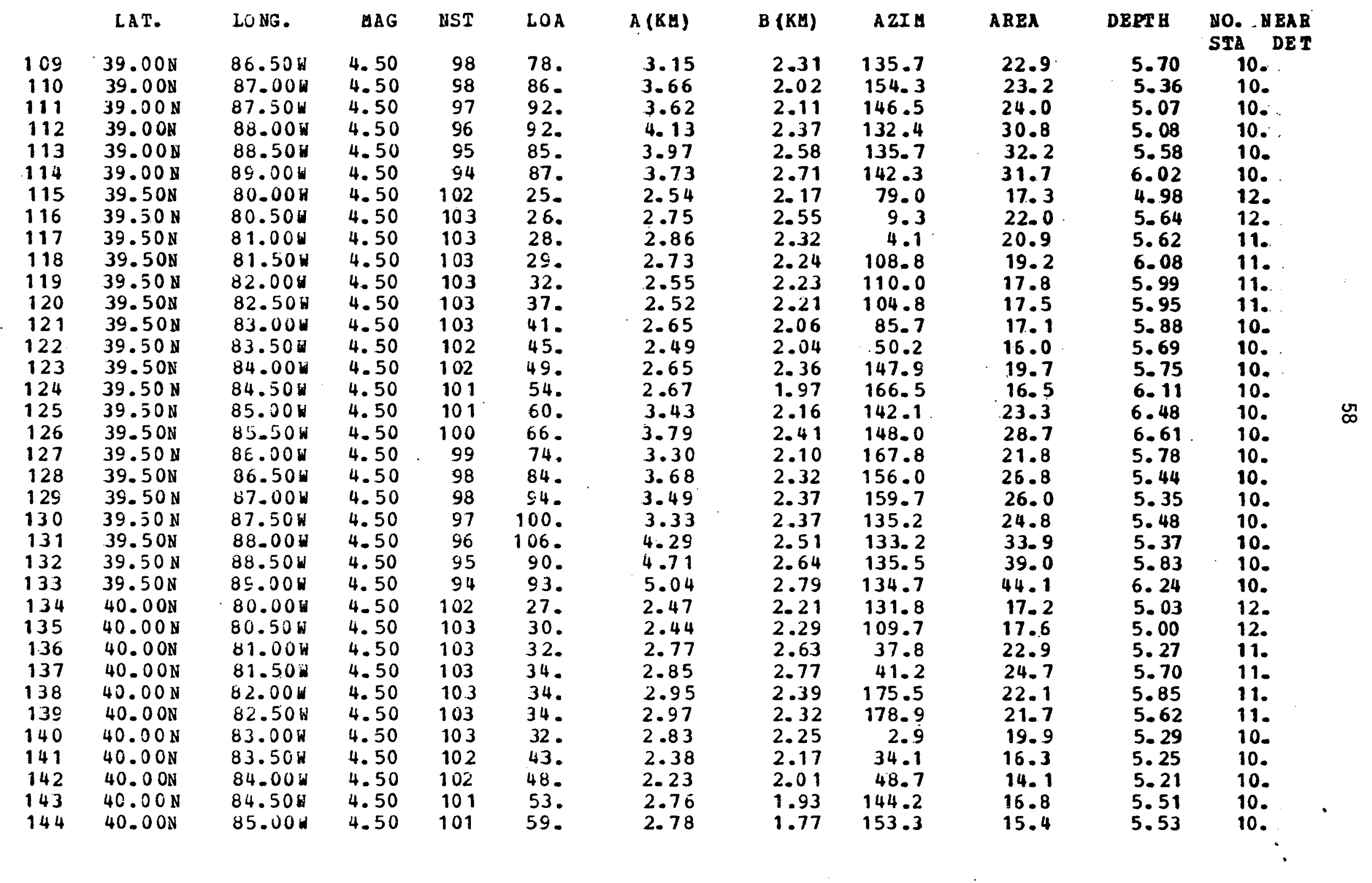




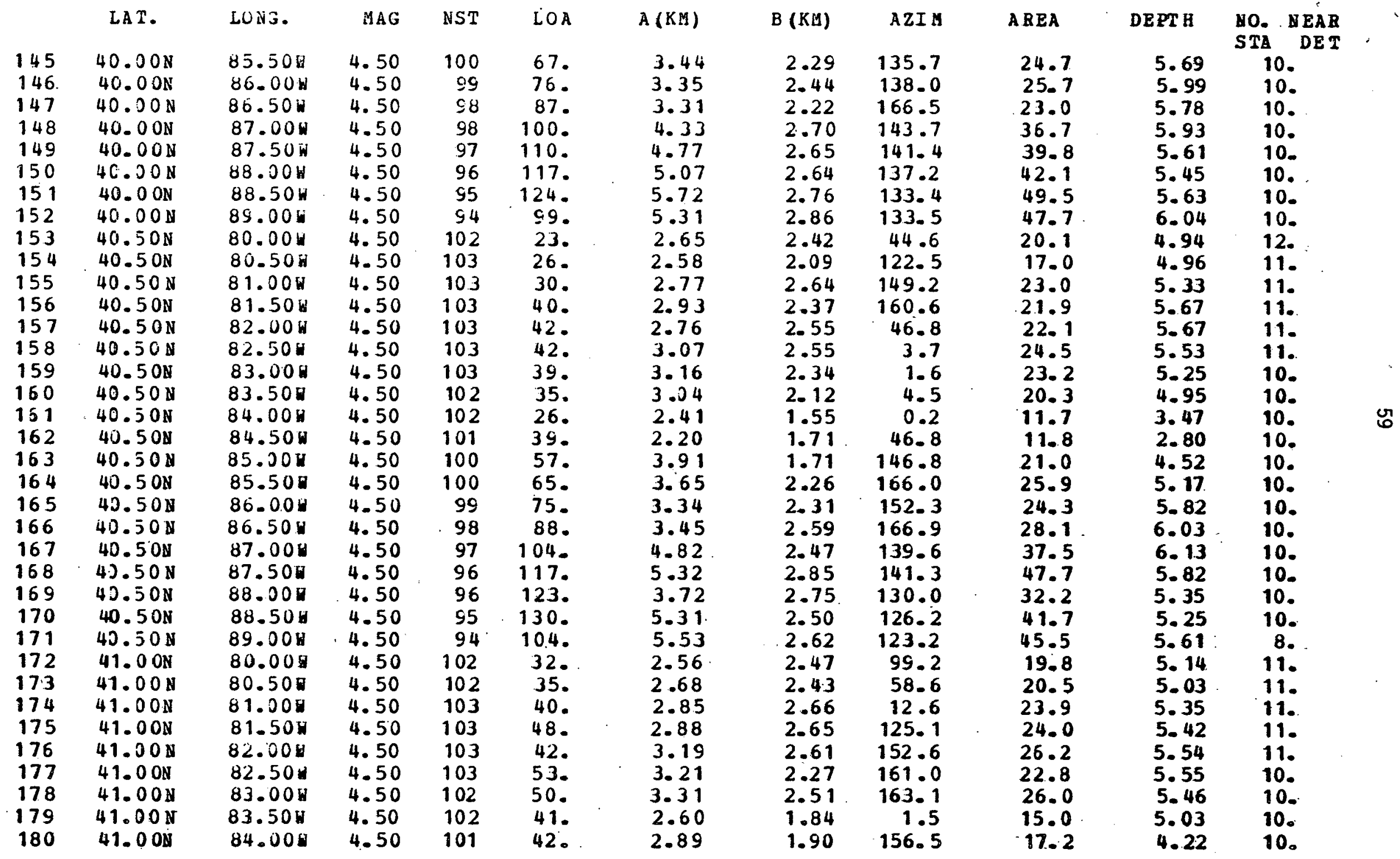




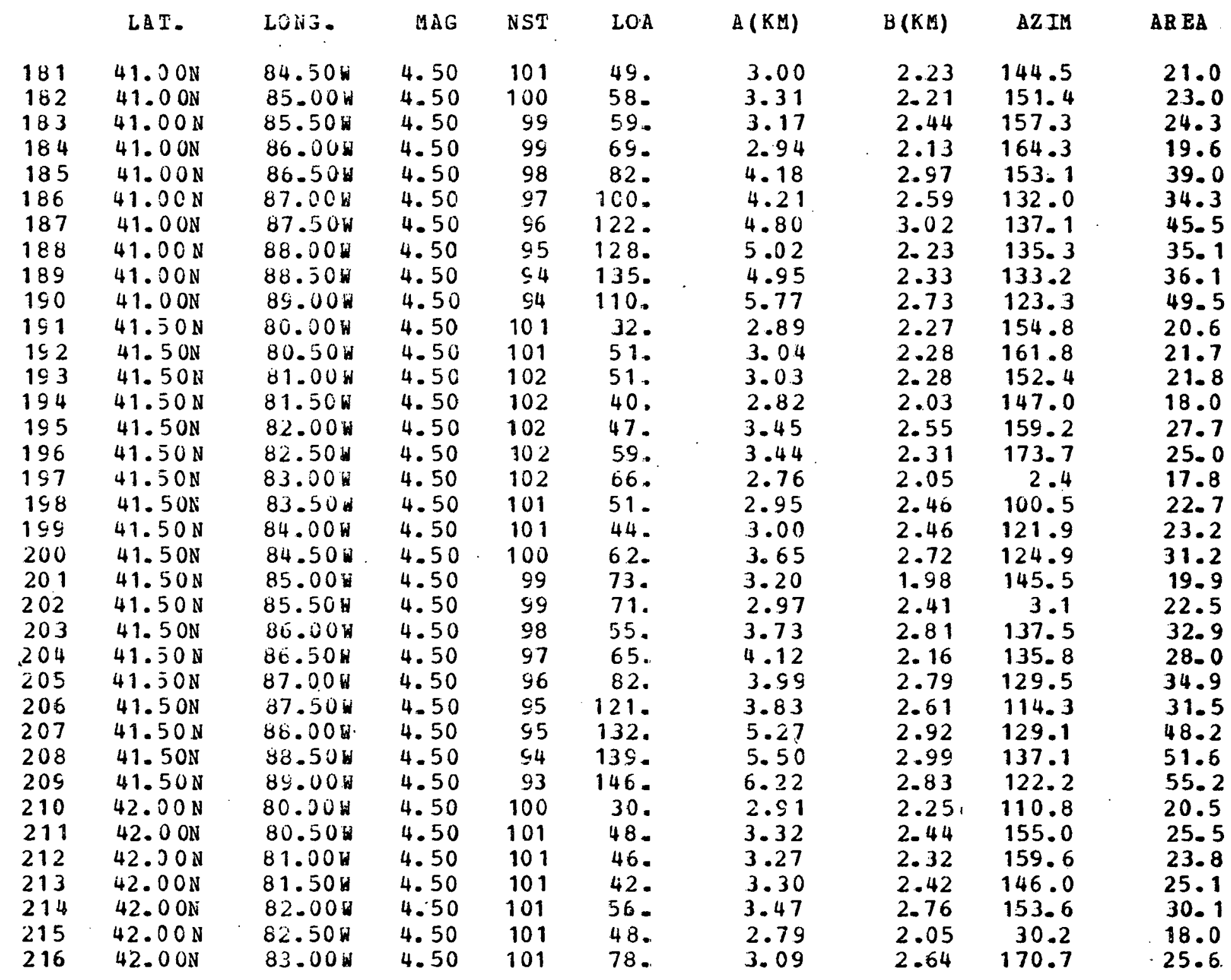

DEPT H

4. 39

4.79

5. 33

5. 78

6. 21

6.07

5. 28

5. 11

5. 12

5. 39

5. 53

5. 42

5. 24

3. 40

5.47

5. 72

5. 59

4. 95

4.69

5.01

5. 28

5.56

5. 88

6.03

5.83

5. 15

4.77

4. 97

5. 25

5. 31

5. 53

5. 54

5.52

5.77

5. 80

5.45
HO. NEAR

STA DET

10.

10.

10.

10.

10.

10.

10.

10.

10.

11.

11.

11.

10.

10

10.

10.

10.

10.

10.

10.

10.

10.

10.

10.

10.

10.

8.

6.

10.

10.

10.

10.

10.

10. 


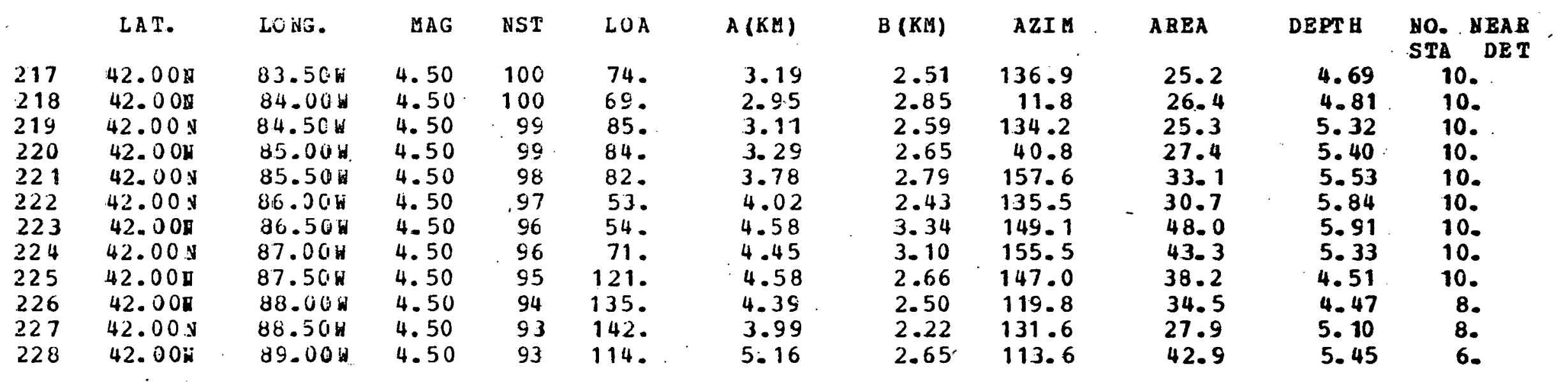


NETHOBR SUAHARY - ERBOB ELLIPSES

(MA GNITUDES BANGE PBOH, 4.00 TO 4.00 IN STEPS OF 0.0 )

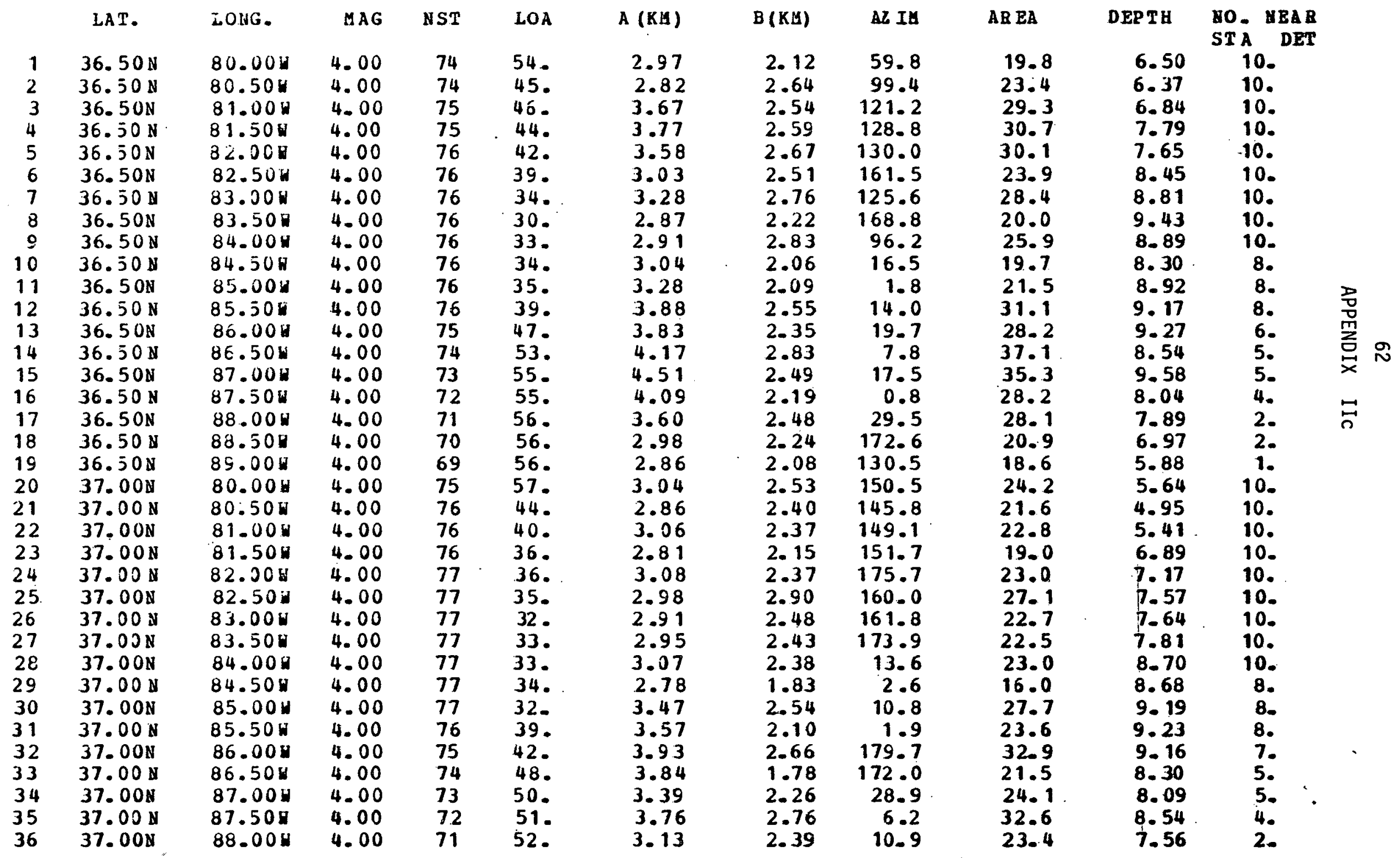




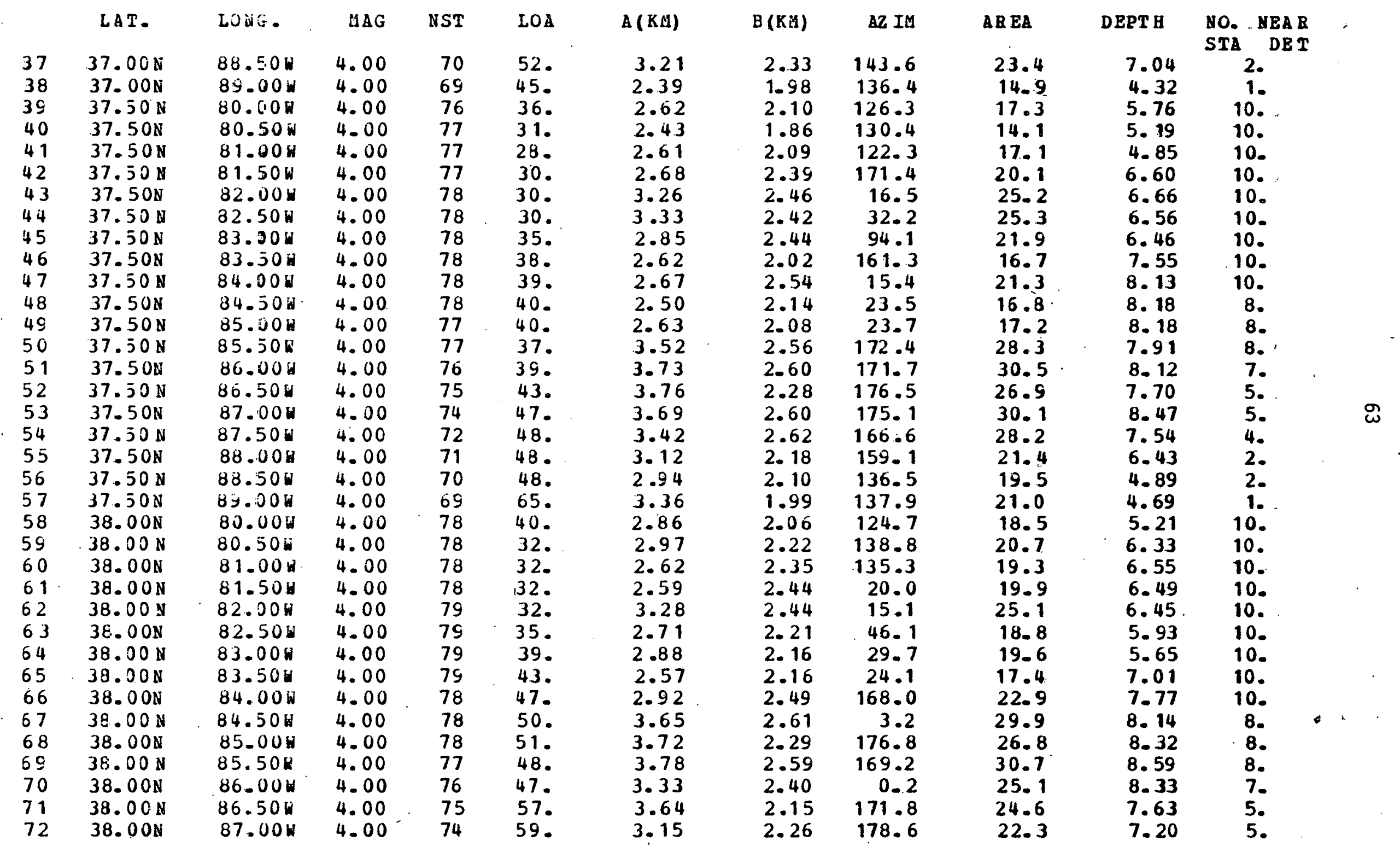




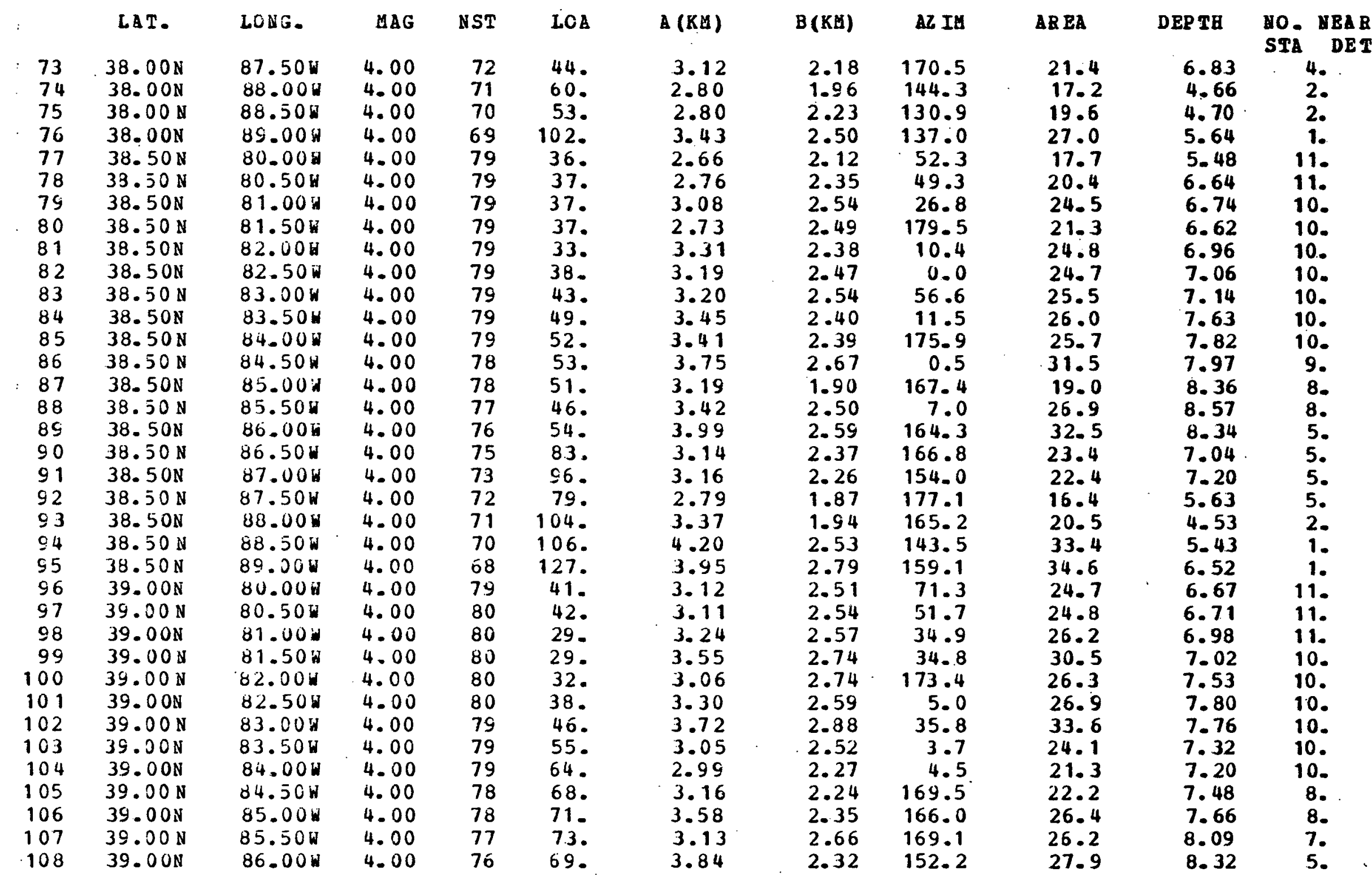




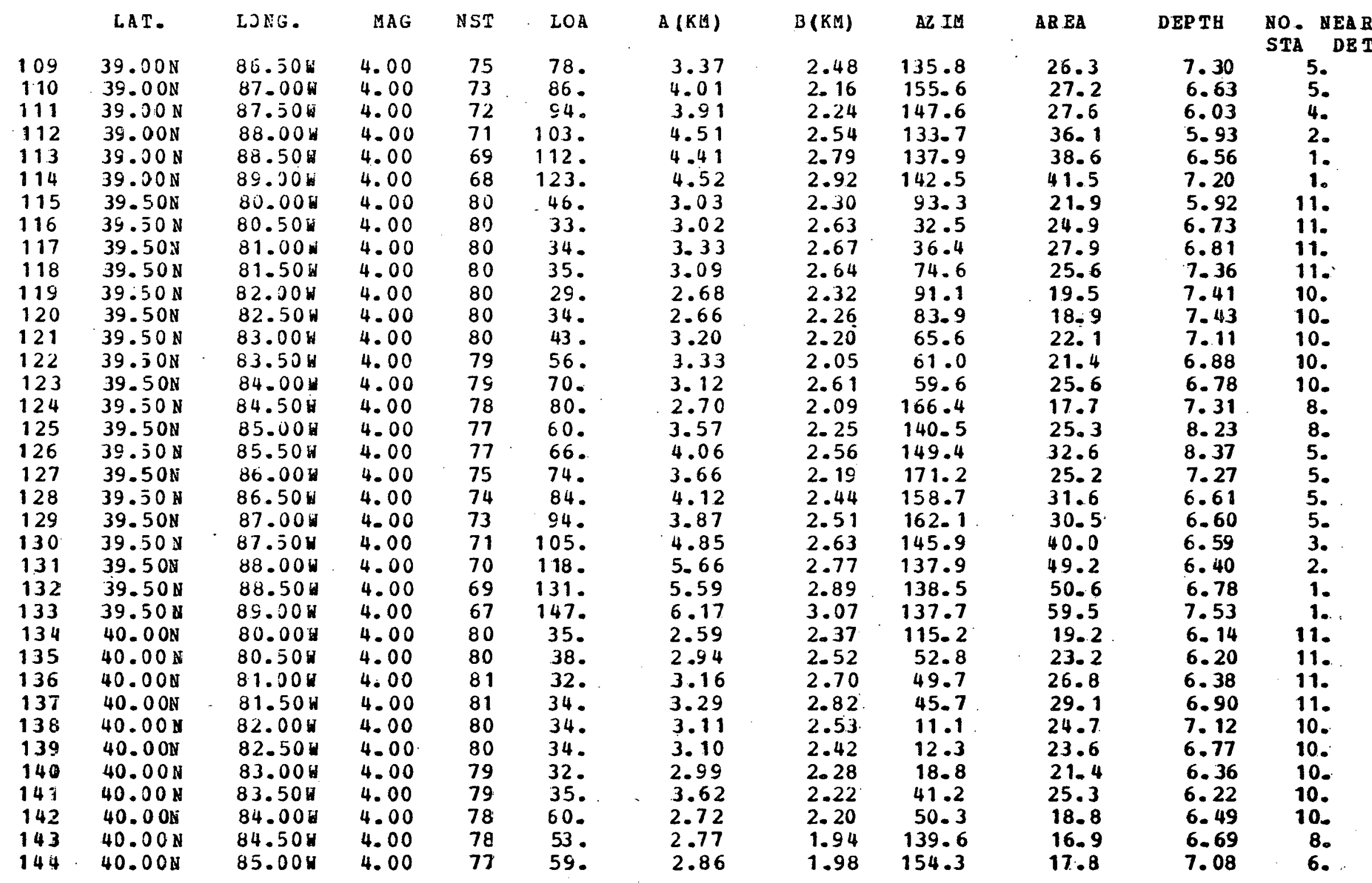




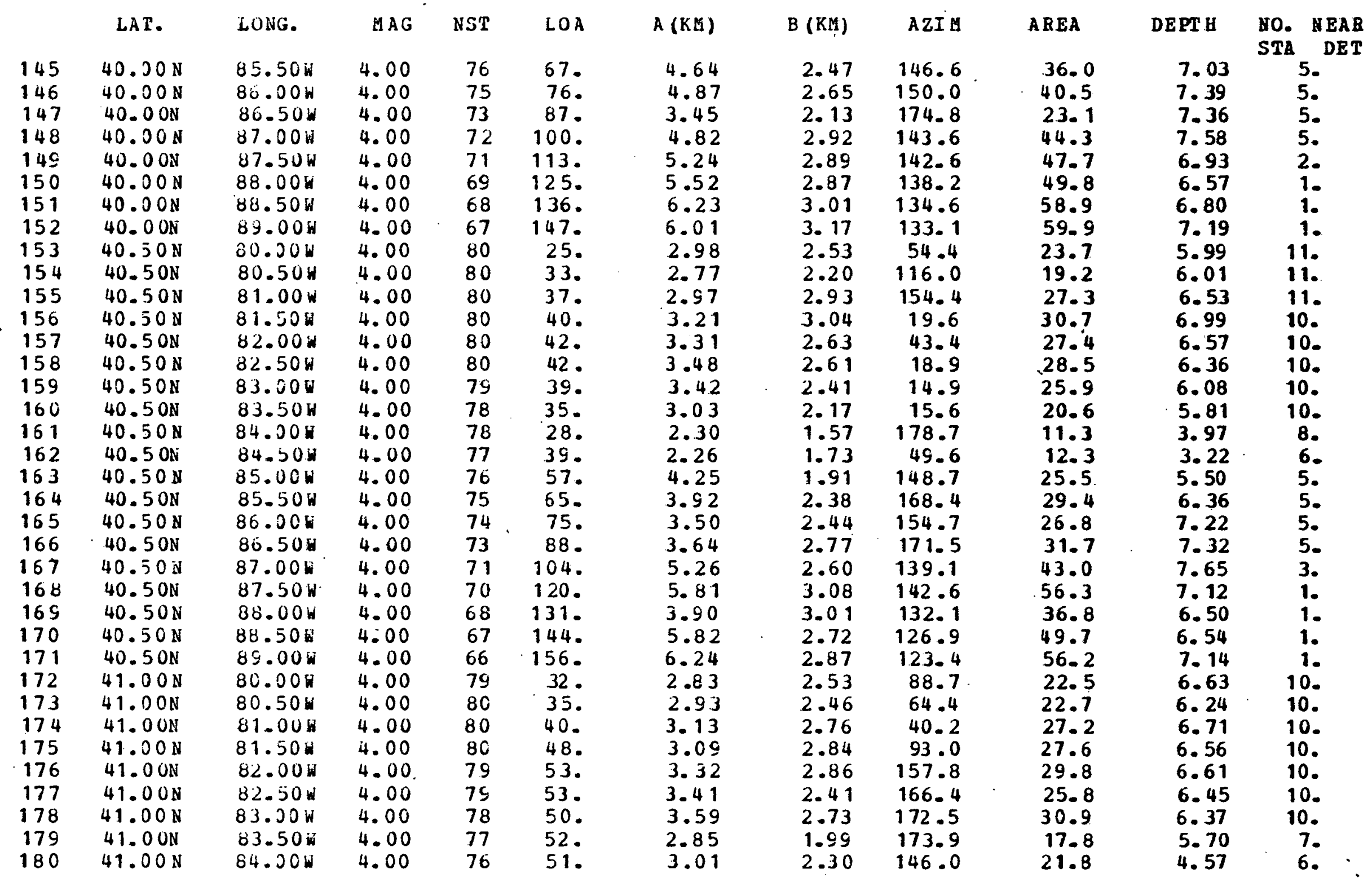




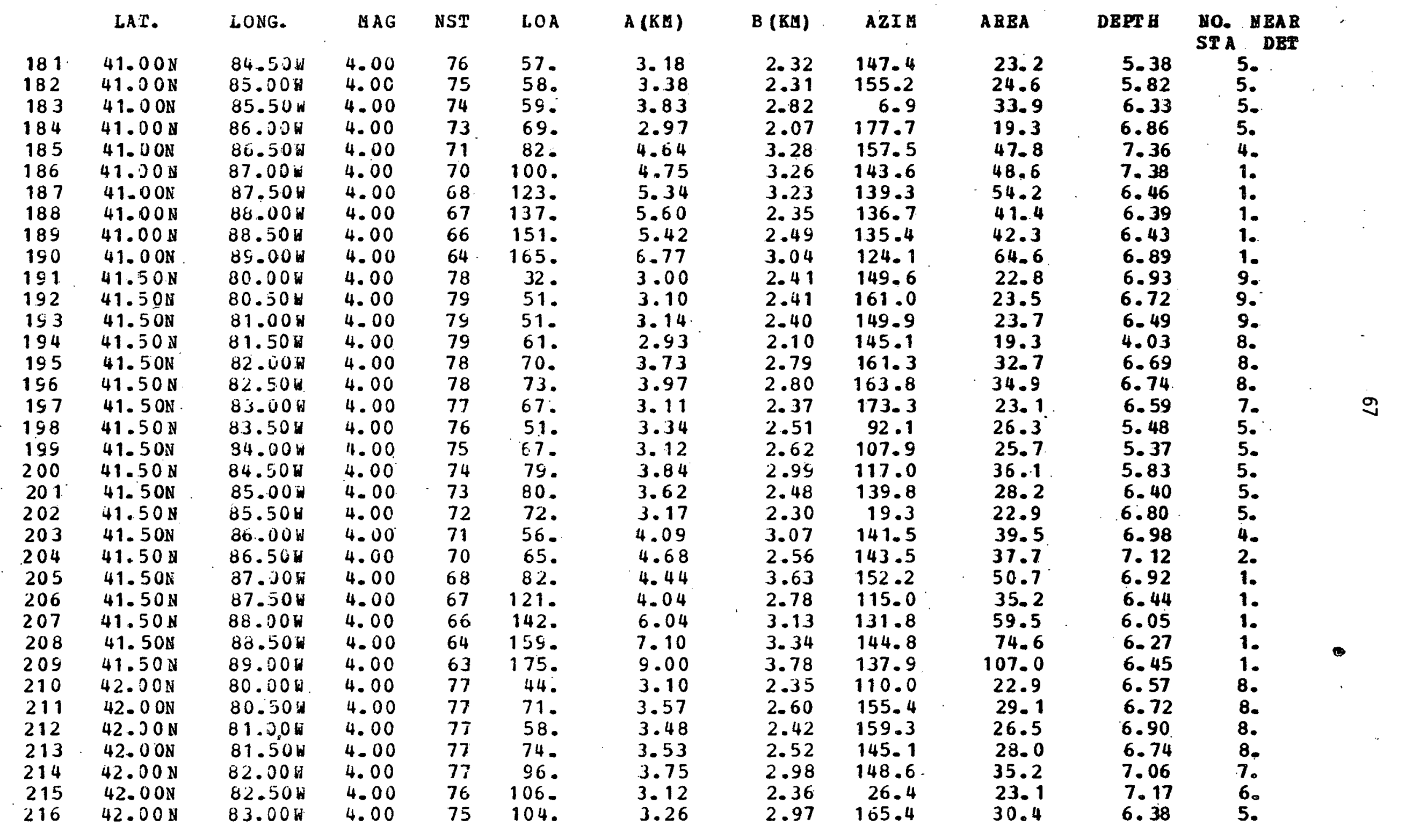




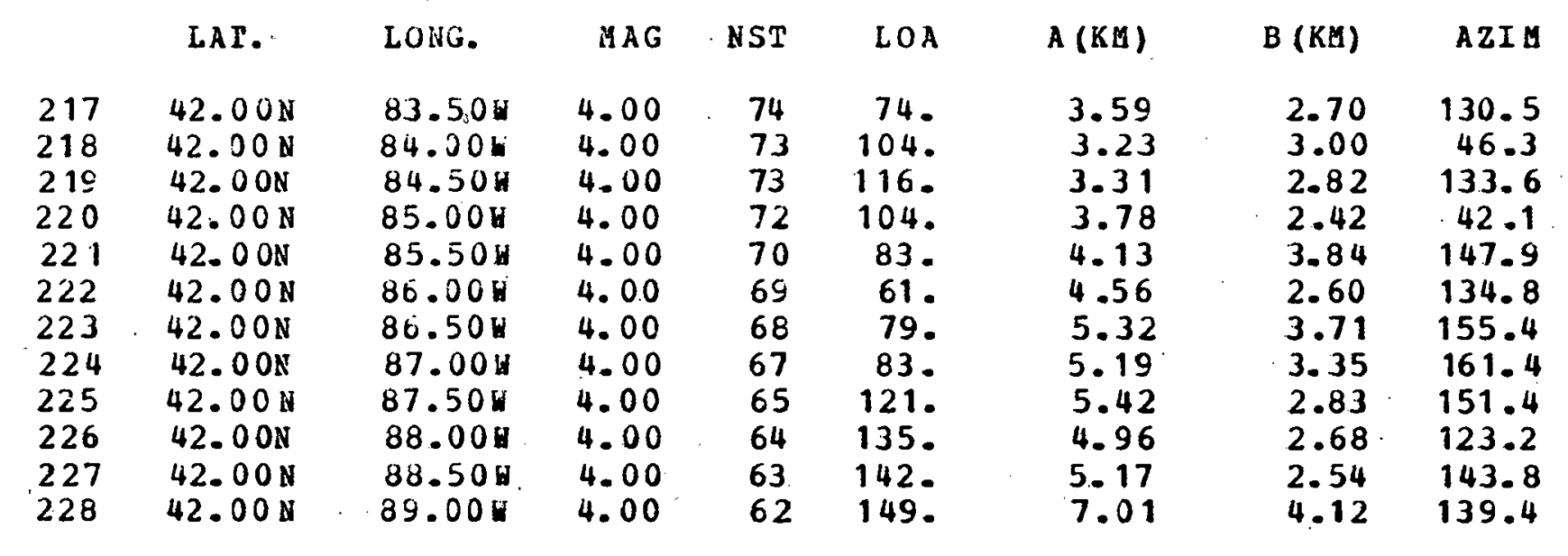

AREA
30.4
30.5
29.4
28.8
49.8
37.3
62.1
54.6
48.3
41.8
41.3
90.8

DEPT

5. 39

5.61

6.26

6.58

6.68

7.24

7.03

6.28

5.20

5.74

6.40

6.77

NO. NEAE

ST A DET

5.

5.

5.

4.

3.

1.

1.

1.

1.

1. 
NETHORK SUMMARY - ERROR ELLIPSES

IMAGNITUDES RANGE FROM, 3.50 TO 3.50 IN STEPS OF 0.0 )

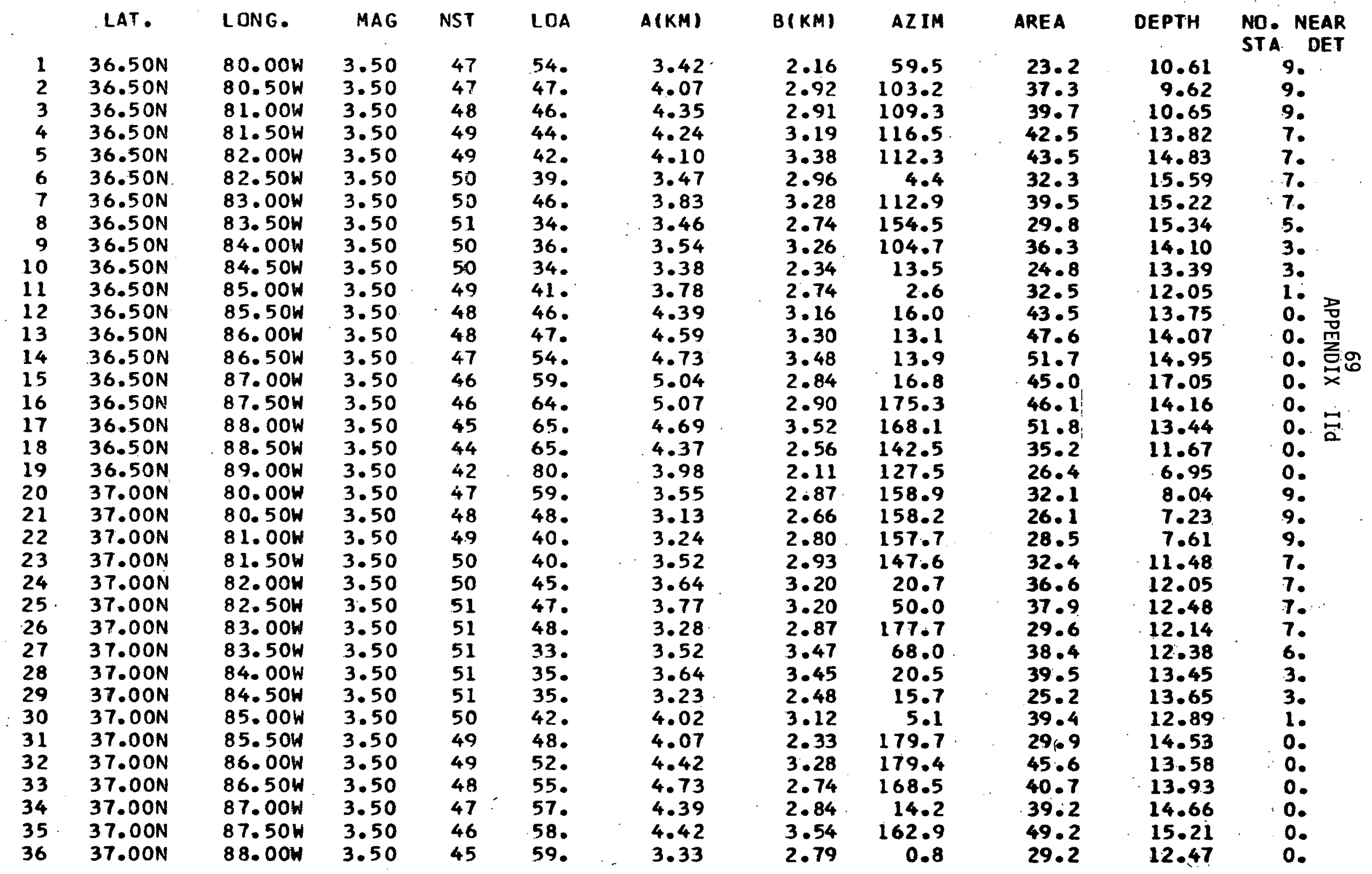




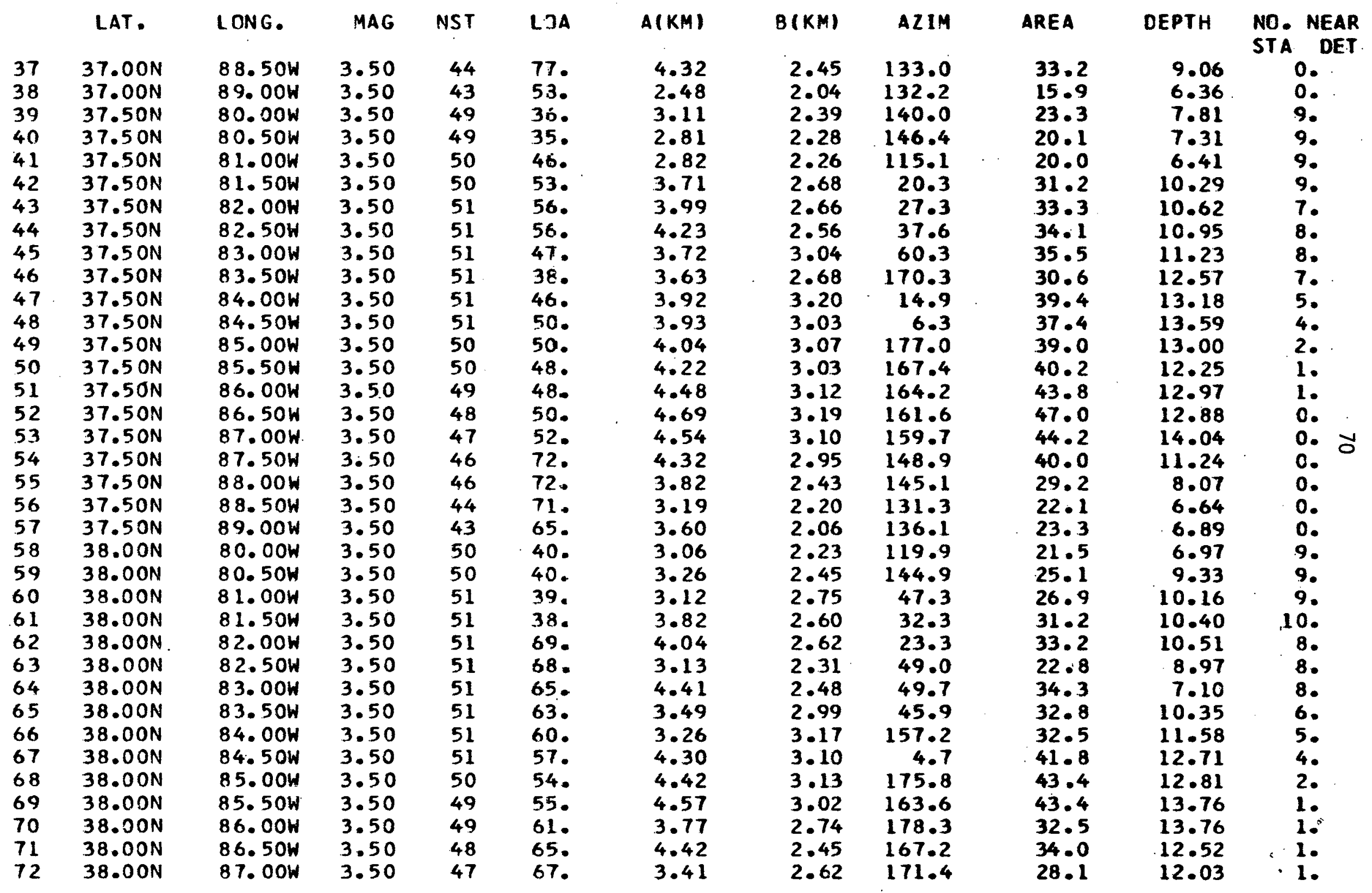




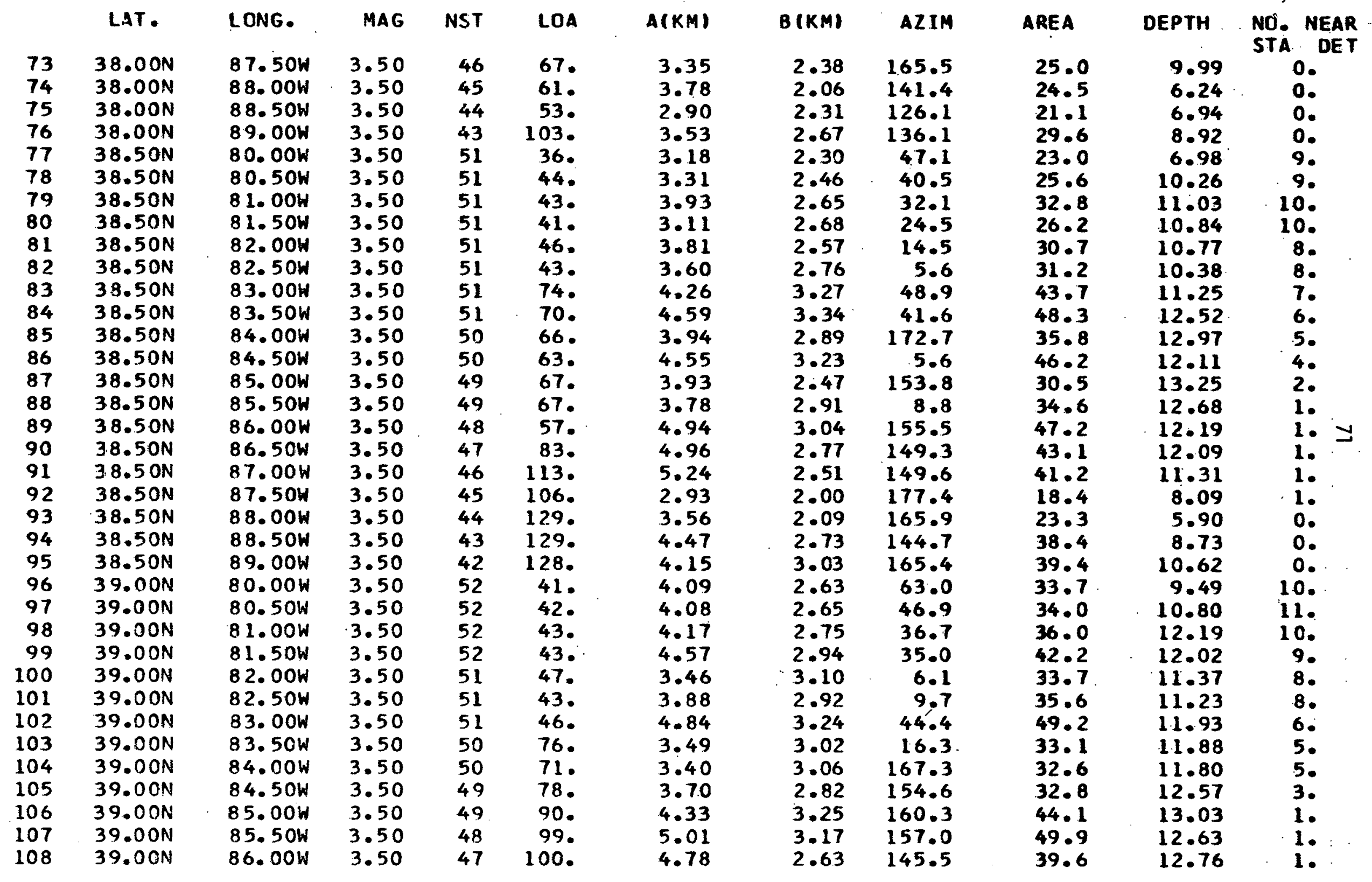




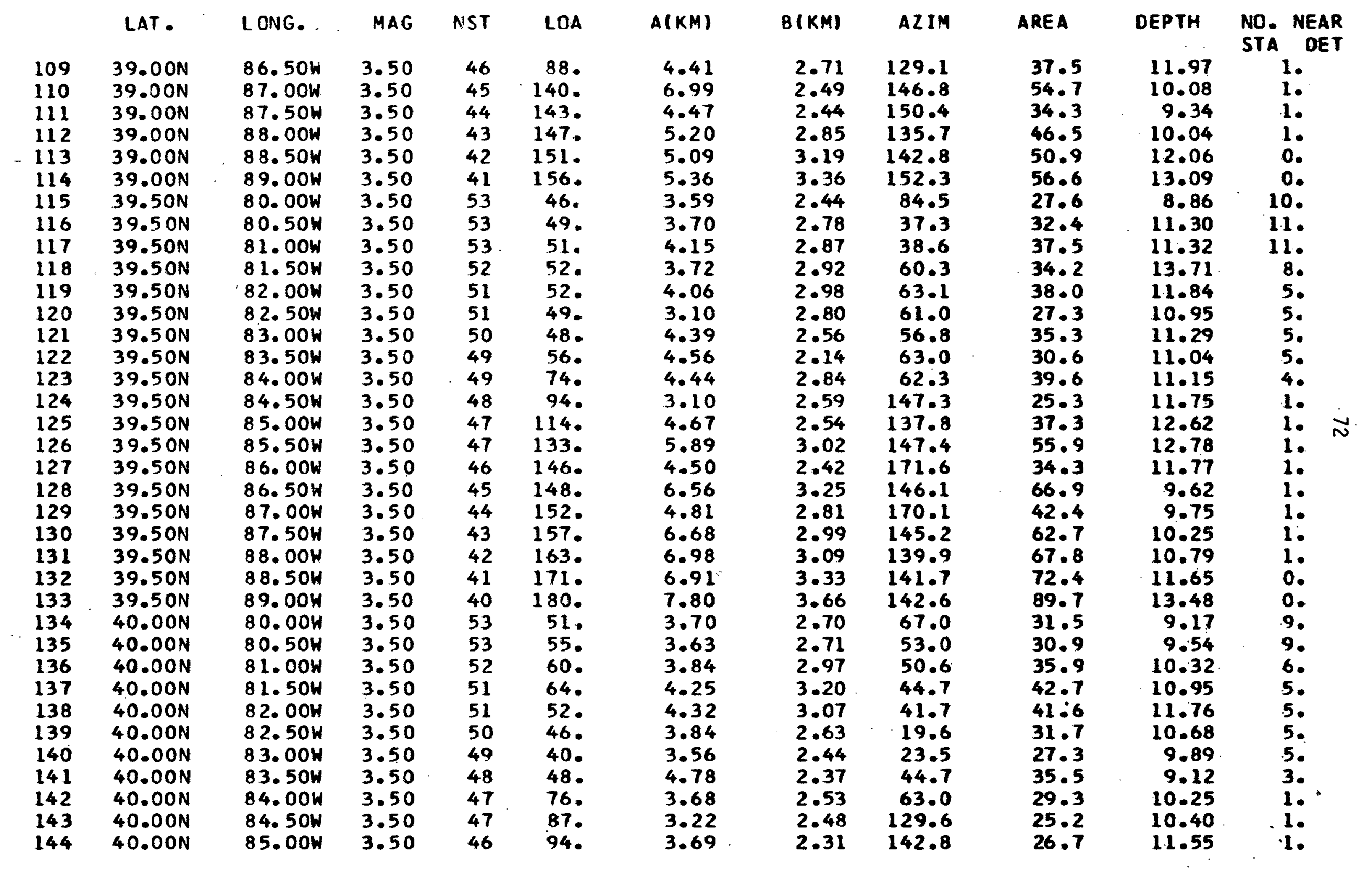




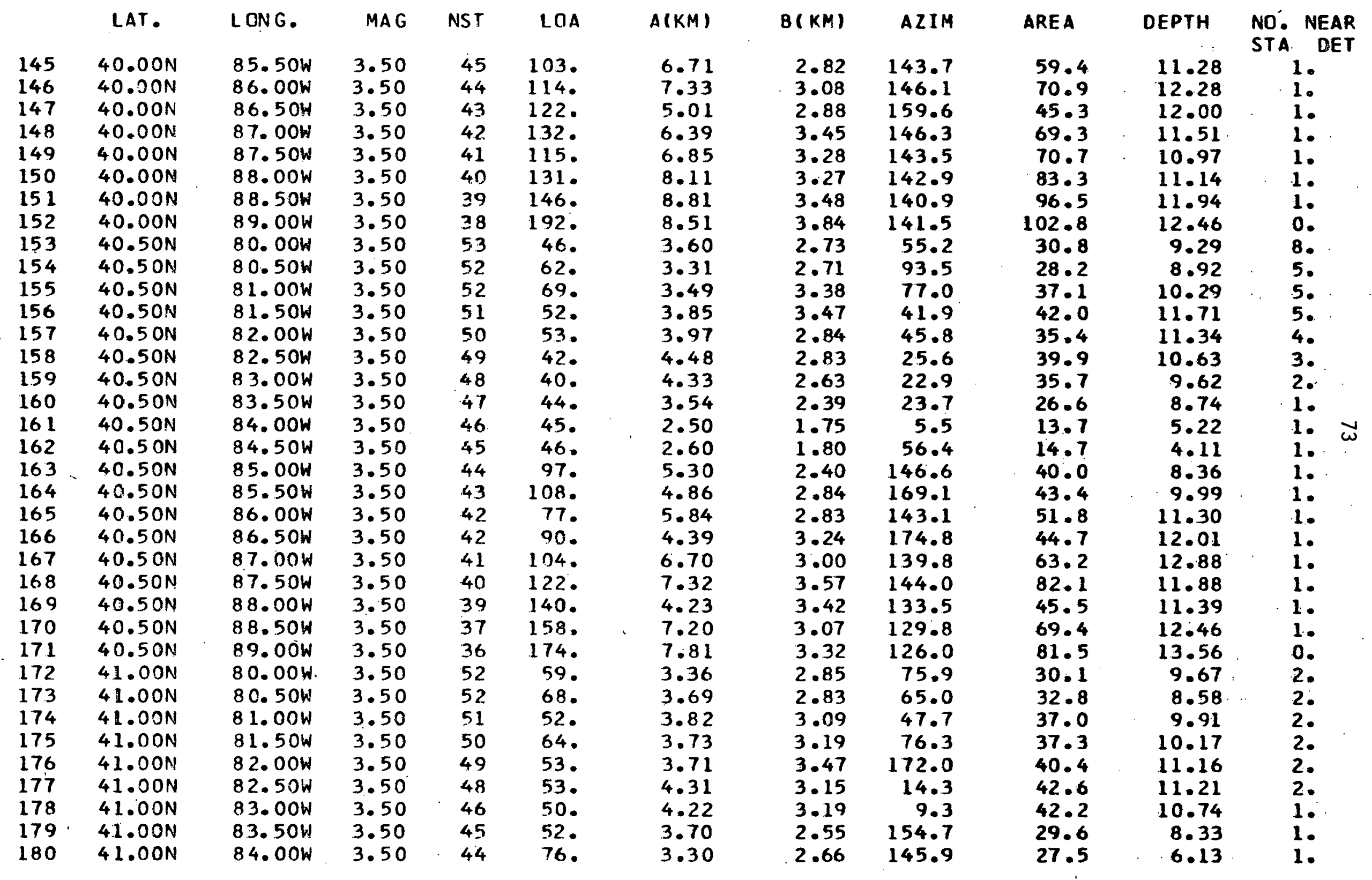




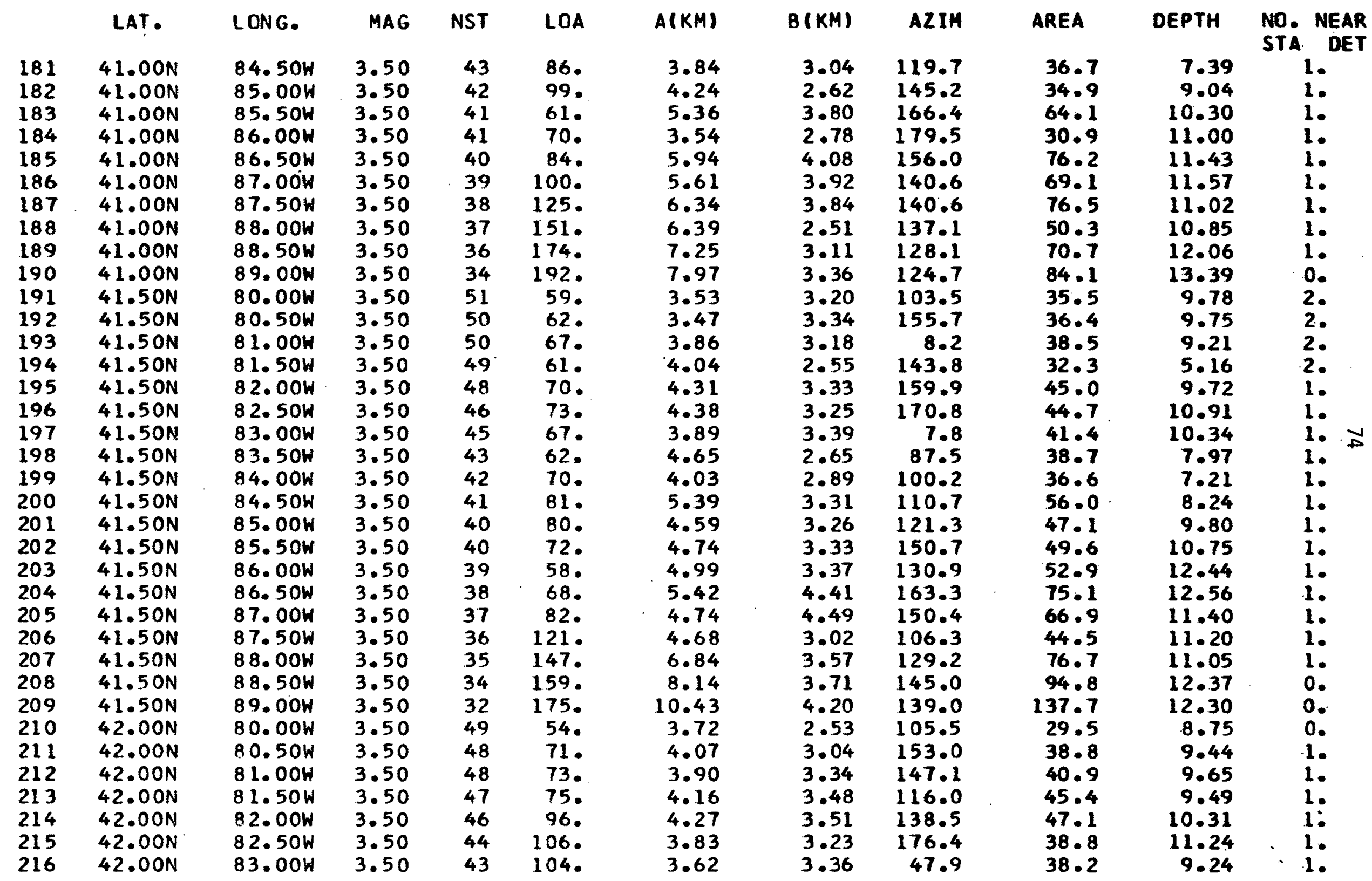




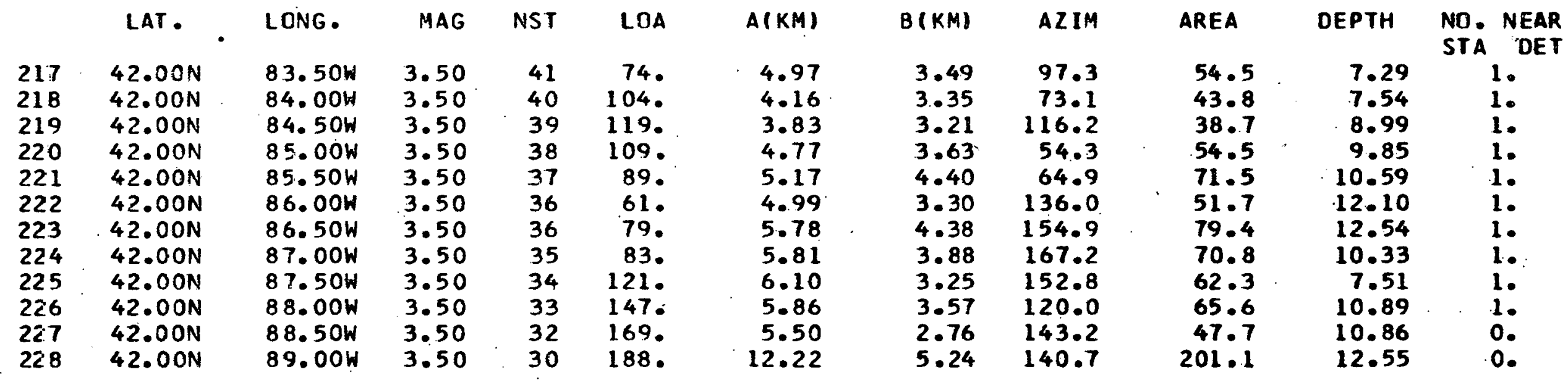


NETHOBK SUEHARY - ERBOB BLLIPSES

(MA GN ITUDES RANGE FROA, 3.00 TO 3.00. IN ST EPS OF 0.0 )

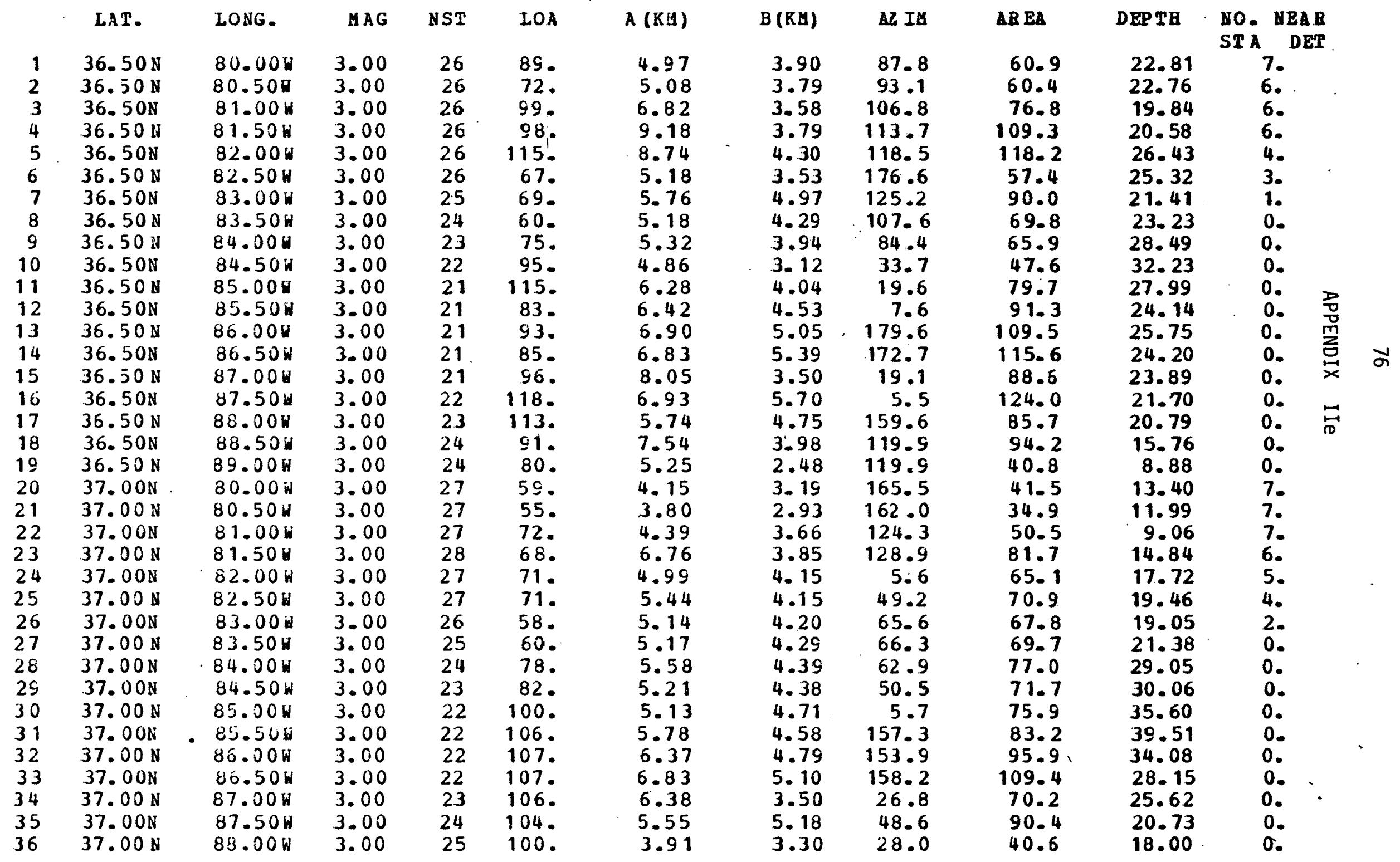




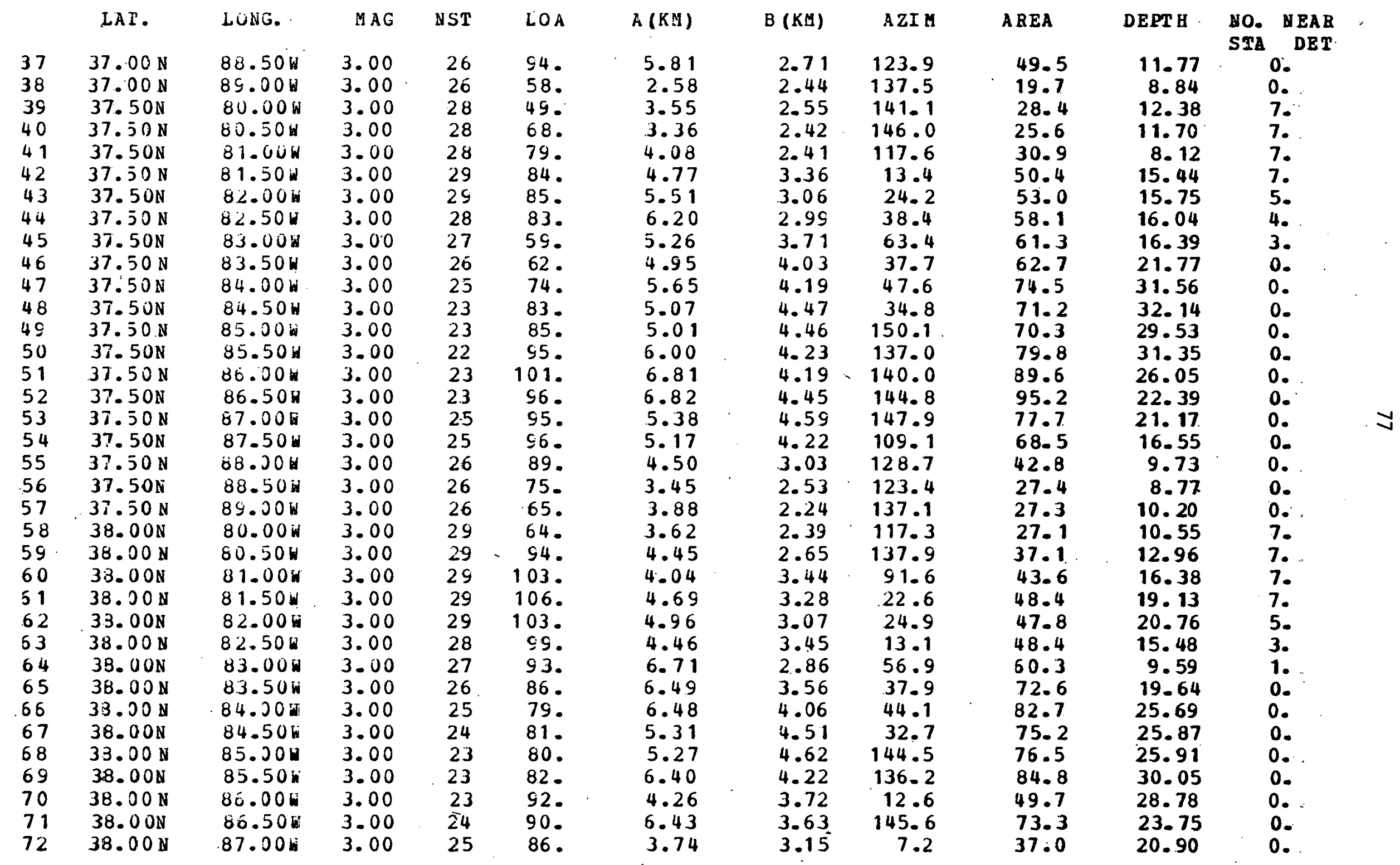




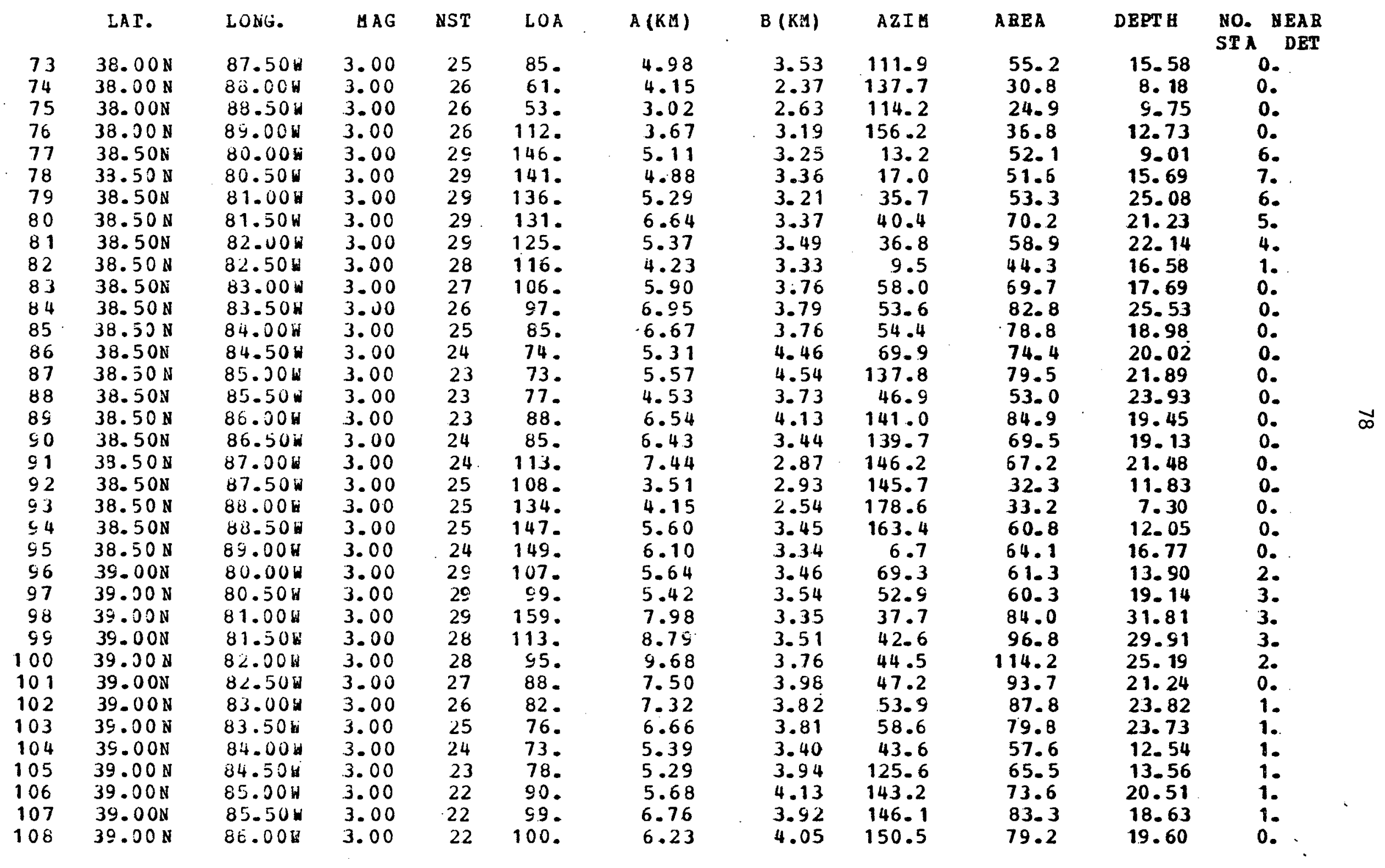




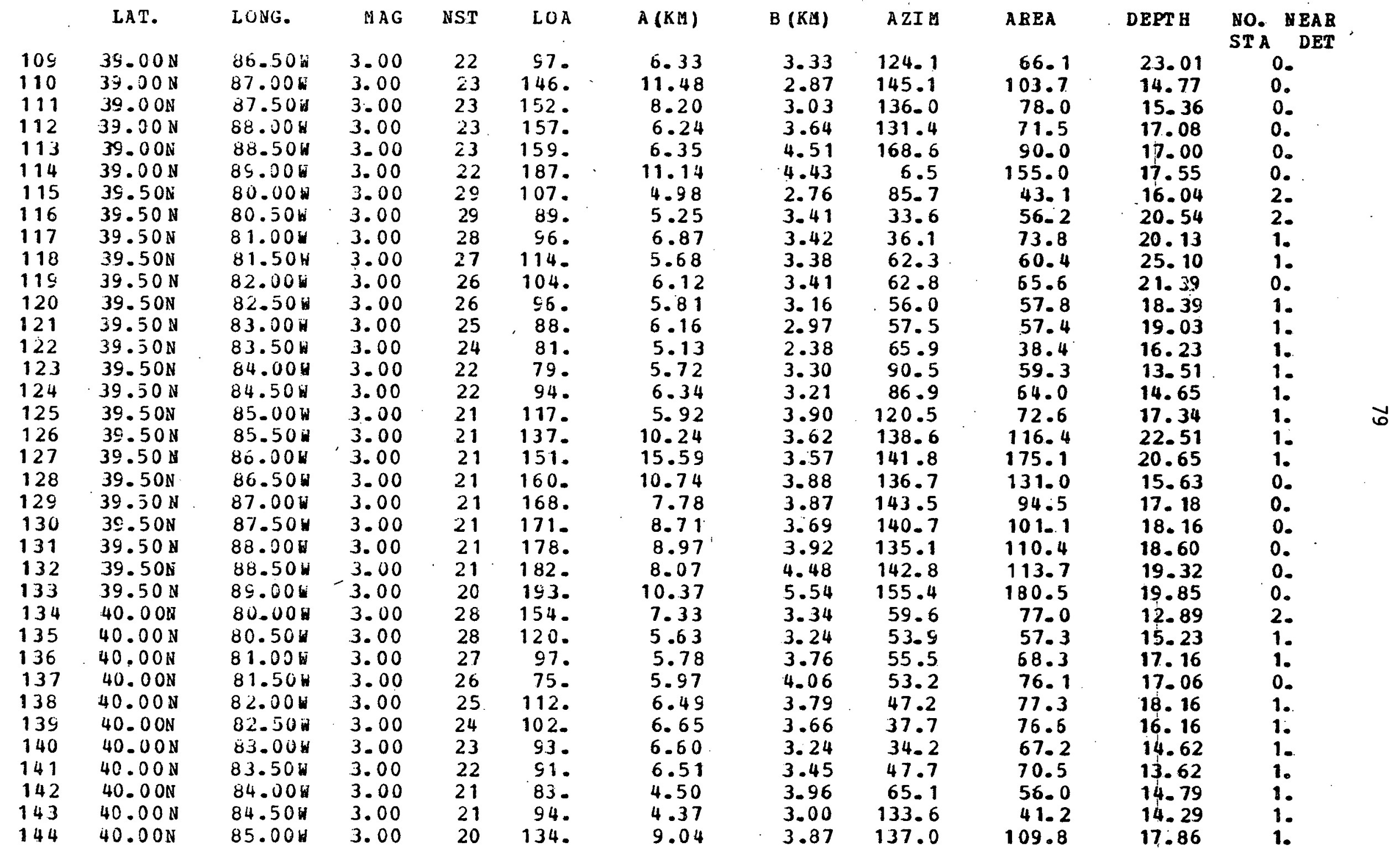




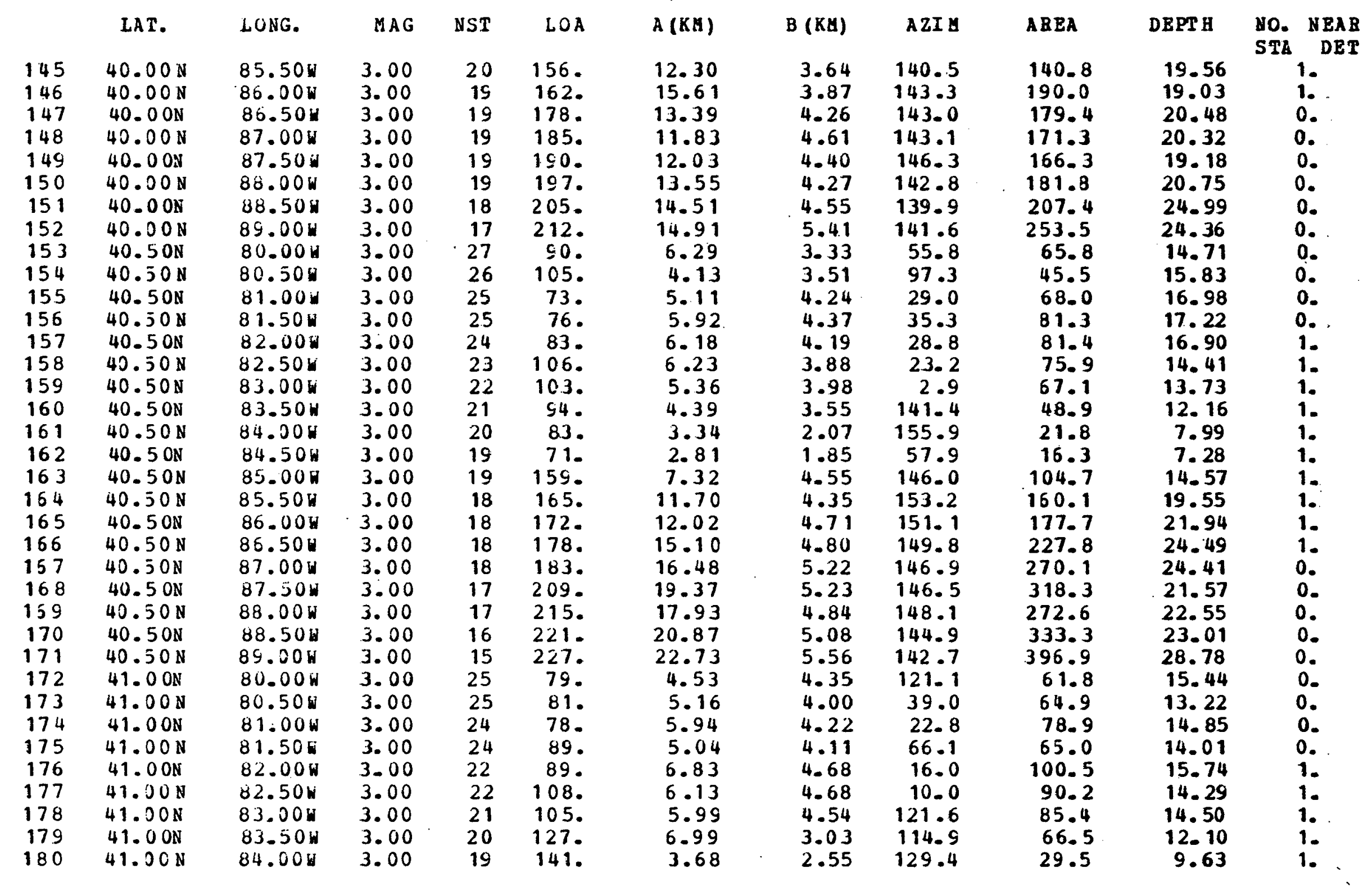




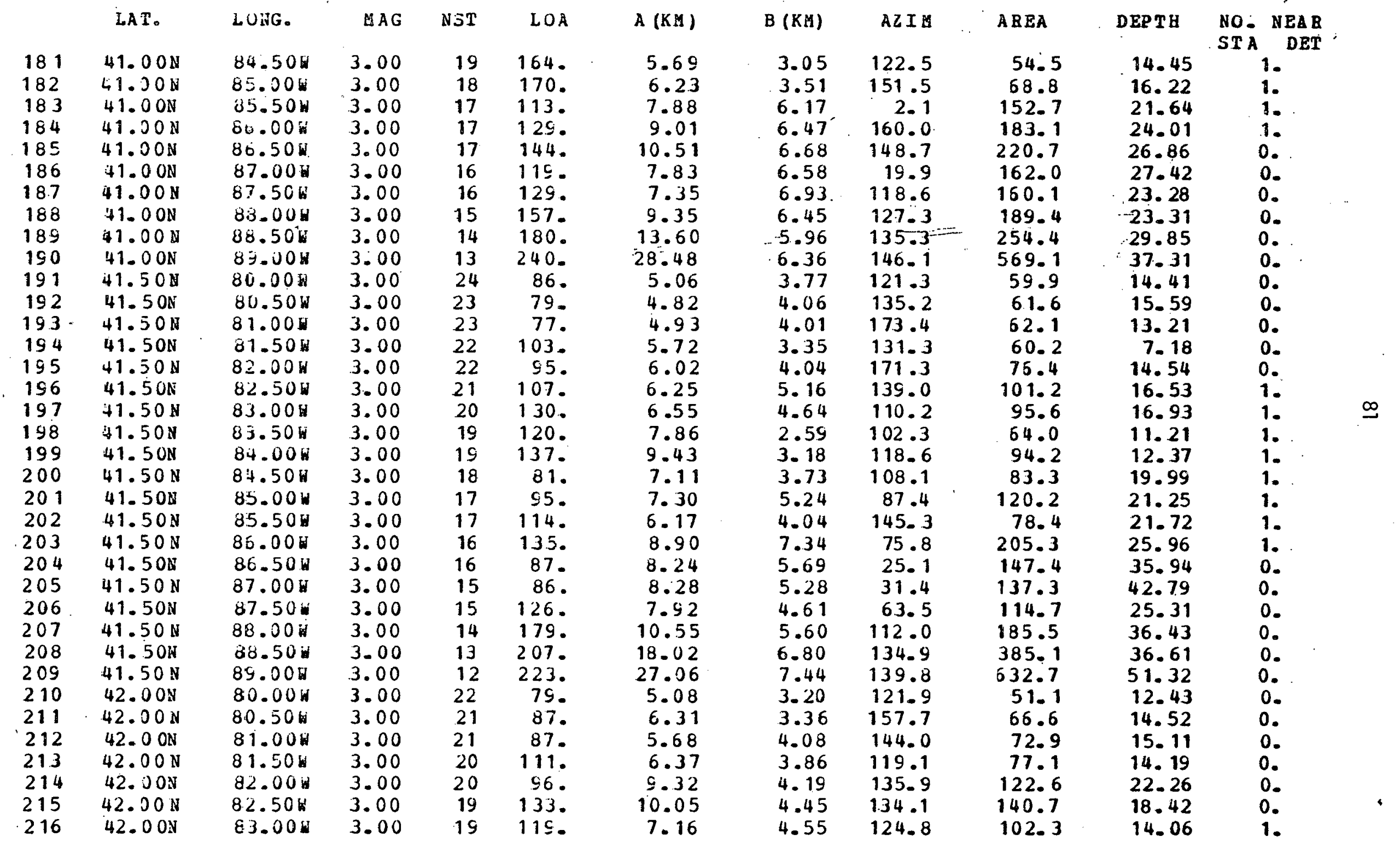




\begin{tabular}{|c|c|c|c|c|c|c|c|c|c|c|c|}
\hline . & LAT. & LONS. & $M A G$ & NST & LOA & $A(K B)$ & $B(K: A)$ & AZIE & $\mathbf{A R} \mathbf{E A}$ & DEPT H & $\begin{array}{l}\text { BO. } \\
\text { STA }\end{array}$ \\
\hline $\begin{array}{l}217 \\
218 \\
219 \\
220 \\
221 \\
222 \\
223 \\
224 \\
225 \\
226 \\
227 \\
228\end{array}$ & $\begin{array}{l}42.00 \mathrm{~N} \\
42.0 \mathrm{~N} \\
42.100 \mathrm{~N} \\
42.0 \mathrm{NN} \\
42.00 \mathrm{~N} \\
42.00 \mathrm{~N} \\
42.00 \mathrm{~N} \\
42.00 \mathrm{~N} \\
42.0 \mathrm{~N} \\
42.00 \mathrm{~N} \\
42.00 \mathrm{~N} \\
42.00 \mathrm{~N}\end{array}$ & $\begin{array}{l}83.50 \mathrm{~W} \\
84.00 \mathrm{~W} \\
84.50 \mathrm{~W} \\
85.0 \mathrm{~W} \\
85.50 \mathrm{~W} \\
86.00 \mathrm{~W} \\
86.50 \mathrm{~W} \\
87.00 \mathrm{~W} \\
87.50 \mathrm{~W} \\
88.00 \mathrm{~W} \\
88.50 \mathrm{~W} \\
89.00 \mathrm{~W}\end{array}$ & $\begin{array}{l}3.00 \\
3.00 \\
3.00 \\
3.00 \\
3.00 \\
3.00 \\
3.00 \\
3.00 \\
3.00 \\
3.00 \\
3.00 \\
3.00\end{array}$ & $\begin{array}{l}18 \\
18 \\
17 \\
17 \\
16 . \\
15 \\
15 \\
14 \\
13 \\
13 \\
12 \\
10\end{array}$ & $\begin{array}{l}108 . \\
104 . \\
119 . \\
109 . \\
109 . \\
104 . \\
133 . \\
154 . \\
121 . \\
153 . \\
175 . \\
252 .\end{array}$ & $\begin{array}{r}9.24 \\
7.07 \\
7.05 \\
6.50 \\
8.80 \\
5.97 \\
7.29 \\
9.23 \\
7.26 \\
8.32 \\
10.79 \\
53.22\end{array}$ & $\begin{array}{l}3.72 \\
4.11 \\
4.92 \\
5.27 \\
4.99 \\
3.91 \\
5.85 \\
4.55 \\
3.81 \\
7.03 \\
7.81 \\
9.08\end{array}$ & $\begin{array}{r}96.9 \\
109.5 \\
115.7 \\
84.3 \\
74.4 \\
132.1 \\
162.4 \\
174.4 \\
157.8 \\
72.2 \\
126.0 \\
149.7\end{array}$ & $\begin{array}{r}107.9 \\
91.3 \\
108.9 \\
107.7 \\
138.0 \\
73.3 \\
134.0 \\
131.9 \\
87.0 \\
183.6 \\
264.7 \\
1518.7\end{array}$ & $\begin{array}{l}10.83 \\
13.53 \\
20.74 \\
28.52 \\
19.79 \\
34.91 \\
31.22 \\
26.00 \\
18.86 \\
25.40 \\
48.88 \\
58.34\end{array}$ & \\
\hline
\end{tabular}


NETWDRK SUMMARY - ERROR ELLIPSES

IMAGNITUDES RANGE FROM, 2.50 TO 2.50 IN STEPS OF 0.01

LAT. LONG. MAG NST $10 \underset{\text { LOA STION MINIMUM COVERAGE }}{\text { MOKM) }}$

\begin{tabular}{ll}
1 & $36.50 \mathrm{~N}$ \\
2 & $36.50 \mathrm{~N}$ \\
3 & $36.50 \mathrm{~N}$ \\
4 & $36.50 \mathrm{~N}$ \\
5 & $36.50 \mathrm{~N}$ \\
6 & $36.50 \mathrm{~N}$ \\
7 & $36.50 \mathrm{~N}$ \\
8 & $36.5 \mathrm{~N}$ \\
9 & $36.50 \mathrm{~N}$ \\
10 & $36.50 \mathrm{~N}$ \\
11 & $36.50 \mathrm{~N}$ \\
12 & $36.50 \mathrm{~N}$ \\
13 & $36.50 \mathrm{~N}$ \\
14 & $36.50 \mathrm{~N}$ \\
15 & $36.50 \mathrm{~N}$ \\
16 & $36.50 \mathrm{~N}$ \\
17 & $36.50 \mathrm{~N}$ \\
18 & $36.50 \mathrm{~N}$ \\
19 & $36.50 \mathrm{~N}$ \\
20 & $37.00 \mathrm{~N}$ \\
21 & $37.0 \mathrm{~N}$ \\
22 & $37.00 \mathrm{~N}$ \\
23 & $37.00 \mathrm{~N}$ \\
24 & $37.0 \mathrm{~N}$ \\
25 & $37.00 \mathrm{~N}$ \\
26 & $37.0 \mathrm{~N}$ \\
27 & $37.00 \mathrm{~N}$ \\
28 & $37.00 \mathrm{~N}$ \\
29 & $37.00 \mathrm{~N}$ \\
30 & $37.00 \mathrm{~N}$ \\
31 & $37.00 \mathrm{~N}$ \\
32 & $37.00 \mathrm{~N}$ \\
33 & $37.00 \mathrm{~N}$ \\
34 & $37.00 \mathrm{~N}$ \\
35 & $37.0 \mathrm{~N}$ \\
36 & $37.00 \mathrm{~N}$ \\
& \\
\hline
\end{tabular}

\begin{tabular}{|c|c|c|c|c|}
\hline LONG. & MAG & NST & LOA & $A(K M)$ \\
\hline $80.00 \mathrm{~W}$ & 2.50 & 14 & 89. & 6.06 \\
\hline $80.50 \mathrm{~W}$ & 2.50 & 13 & 120 & 6.99 \\
\hline $81.00 \mathrm{~W}$ & 2.50 & 12 & 152. & 11.03 \\
\hline $81.50 \mathrm{~W}$ & 2.50 & 11 & 187. & 17.19 \\
\hline $82.00 \mathrm{~W}$ & 2.50 & 10 & 147 . & 13.49 \\
\hline $82.50 \mathrm{~W}$ & 0.77 & 0 & 0 & 0.0 \\
\hline $83.00 \mathrm{~W}$ & 0.57 & 0 & 0. & 0.0 \\
\hline $83.50 \mathrm{w}$ & 0.45 & 0 & 0. & 0.0 \\
\hline $84.00 \mathrm{~W}$ & 0.34 & 0 & 0. & 0.0 \\
\hline $84.50 \mathrm{~W}$ & 0.24 & 0 & 0. & 0.0 \\
\hline $85.00 w$ & 0.22 & D & 0. & 0.0 \\
\hline $85.50 \mathrm{~W}$ & 0.24 & 0 & 0. & 0.0 \\
\hline $86.00 \mathrm{~W}$ & 0.29 & 0 & 0. & 0.0 \\
\hline $86.50 \mathrm{~W}$ & 0.38 & $D$ & 0. & 0.0 \\
\hline $87: 00 \mathrm{~W}$ & 0.55 & 0 & 0. & 0.0 \\
\hline $87.50 \mathrm{~W}$ & 0.76 & D & 0. & 0.0 \\
\hline $89.00 W$ & 2.50 & 10 & 258. & 22.86 \\
\hline $88.50 \mathrm{~W}$ & 2.50 & 12 & 225 . & 12.10 \\
\hline $89.00 \mathrm{~W}$ & 2.50 & 14 & 196. & 6.79 \\
\hline $80.00 \mathrm{~W}$ & 2.50 & 16 & 68. & 4.99 \\
\hline $80.50 W$ & 2.50 & 15 & 70. & 4.25 \\
\hline $81.00 W$ & 2.50 & 14 & 152. & 4.94 \\
\hline $81.50 \mathrm{~W}$ & 2.50 & 12 & 208 & 12.51 \\
\hline $82.00 \mathrm{~W}$ & 2.50 & 11 & 177. & 15.47 \\
\hline $82.50 \mathrm{~W}$ & 2.50 & 10 & 107. & 7.10 \\
\hline $83.00 \mathrm{~W}$ & 0.80 & 0 & 0. & 0.0 \\
\hline $83.50 \mathrm{~W}$ & 0.58 & 0 & 0. & 0.0 \\
\hline $84.00 \mathrm{~W}$ & 0.41 & 0 & 0. & 0.0 \\
\hline $84.50 \mathrm{~W}$ & 0.32 & 0 & 0. & 0.0 \\
\hline $85.00 w$ & 0.29 & 0 & 0. & 0.0 \\
\hline $85.50 \mathrm{~W}$ & 0.3 .1 & 0 & 0. & 0.0 \\
\hline 86. $00 \mathrm{~W}$ & 0.37 & 0 & 0. & 0.0 \\
\hline $86.50 \mathrm{~W}$ & 0.50 & 0 & 0. & 0.0 \\
\hline $87.00 \mathrm{~W}$ & 0.71 & 0 & 0 . & 0.0 \\
\hline $87.50 \mathrm{~W}$ & 2.50 & 10 & 278 & 31.33 \\
\hline $38.00 \mathrm{~W}$ & 2.50 & 12 & 2.36 & 13.15 \\
\hline
\end{tabular}

$\begin{array}{lr}B(K M) & A Z I M \\ 4.82 & 81.0 \\ 4.77 & 82.0 \\ 4.93 & 97.7 \\ 5.64 & 108.7 \\ 6.23 & 122.9 \\ 0.0 & 0.0 \\ 0.0 & 0.0 \\ 0.0 & 0.0 \\ 0.0 & 0.0 \\ 0.0 & 0.0 \\ 0.0 & 0.0 \\ 0.0 & 0.0 \\ 0.0 & 0.0 \\ 0.0 & 0.0 \\ 0.0 & 0.0 \\ 0.0 & 0.0 \\ 7.61 & 102.2 \\ 4.78 & 111.4 \\ 2.73 & 117.0 \\ 3.57 & 168.4 \\ 3.39 & 157.0 \\ 4.47 & 90.0 \\ 4.69 & 120.9 \\ 5.40 & 134.9 \\ 5.24 & 72.3 \\ 0.0 & 0.0 \\ 0.0 & 0.0 \\ 0.0 & 0.0 \\ 0.0 & 0.0 \\ 0.0 & 0.0 \\ 0.0 & 0.0 \\ 0.0 & 0.0 \\ 0.0 & 0.0 \\ 0.0 & 0.0 \\ 9.19 & 90.9 \\ 4.81 & 113.5\end{array}$

AREA

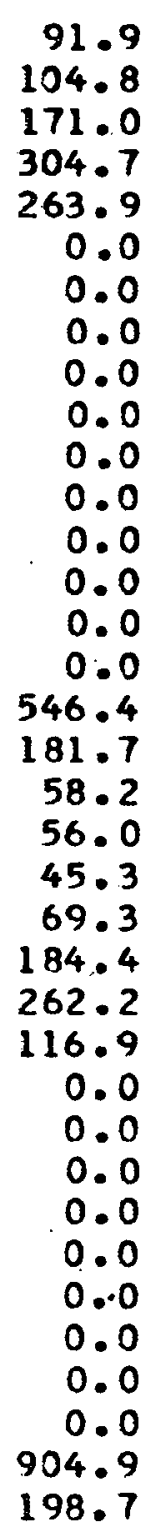

DEPTH

29.26

32.73

34.04

45.49

100.94

0.0

0.0

0.0

0.0

0.0

0.0

0.0

0.0

0.0

0.0

0.0

83.96

34.78

12.92

16.79

16.40

12.47

23.07

43.62

47.85

0.0

0.0

0.0

0.0

0.0

0.0

0.0

0.0

0.0

63.14

32.98
NO. NEAR

STA. DET

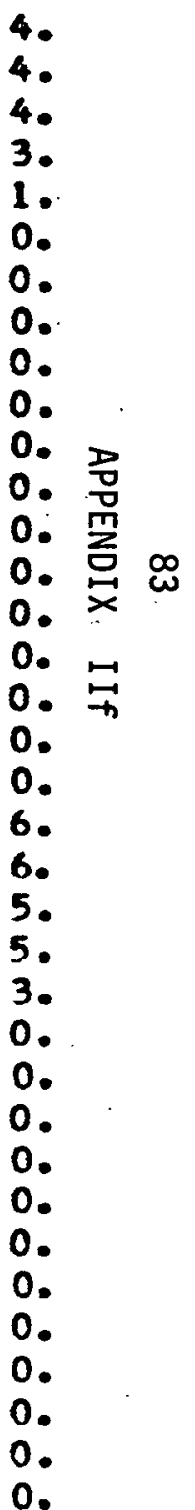




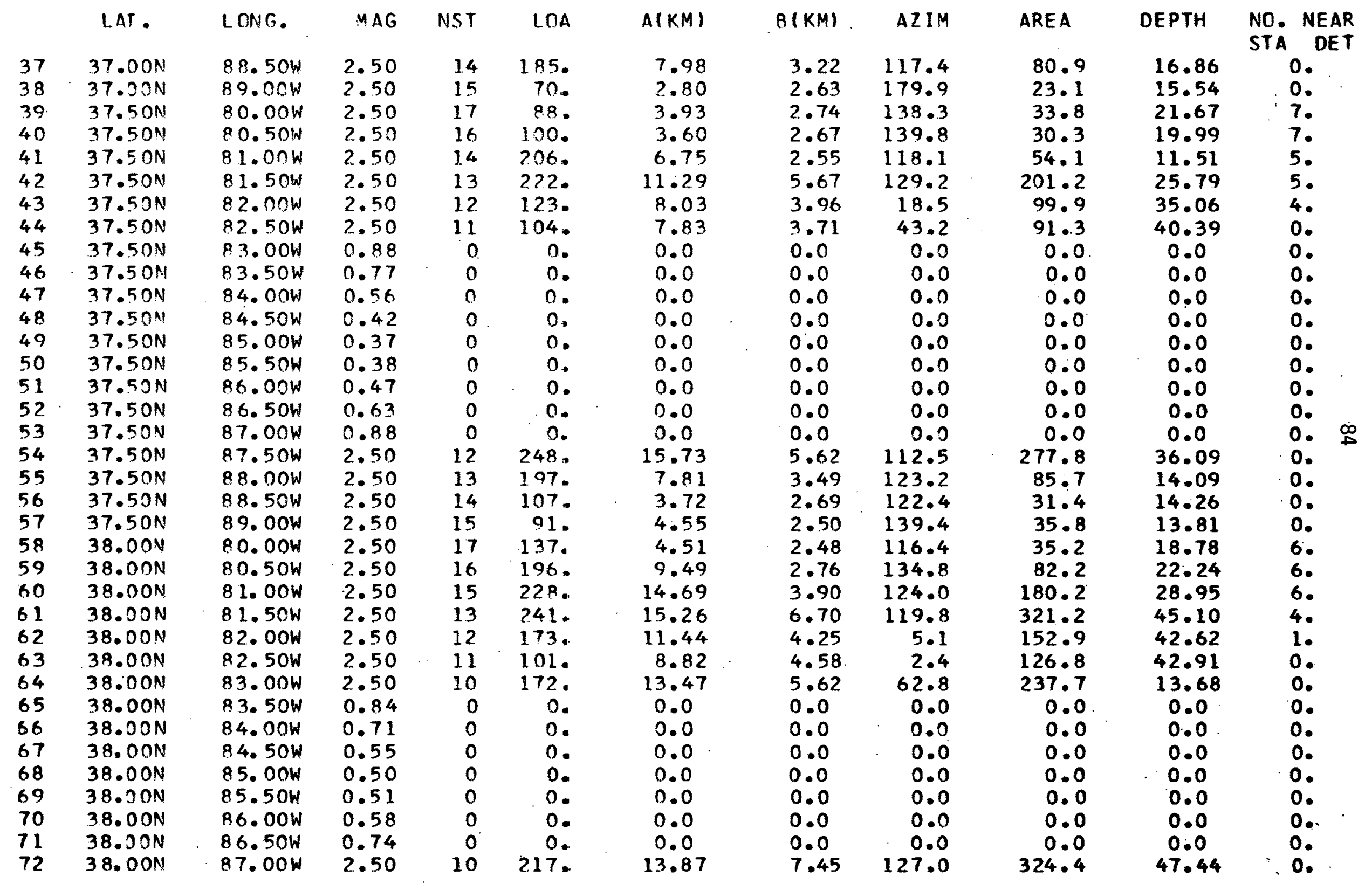




\begin{tabular}{|c|c|c|c|c|c|c|c|c|c|c|c|c|}
\hline & LAT. & LONG. & YAG & NST & LOA & $A(K M)$ & $B(K M)$ & $A Z I M$ & AREA & DEPTH & $\begin{array}{l}\text { NO: } \\
\text { STA }\end{array}$ & $\begin{array}{r}\text { NEAR } \\
\text { DET }\end{array}$ \\
\hline 73 & $38.00 \mathrm{~N}$ & $87.50 \mathrm{~W}$ & 2.50 & 12 & 188 & 8.42 & 4.40 & 110.6 & 116.3 & 22.10 & . & 0. \\
\hline 74 & $38.00 \mathrm{~N}$ & $88.00 \mathrm{~W}$ & 2.50 & 13 & $12 ?$. & 4.53 & 2.50 & 137.8 & 35.6 & 12.84 & & 0. \\
\hline 75 & $38.00 \mathrm{~N}$ & $88.50 \mathrm{~W}$ & 2.50 & 14 & 66. & 3.34 & 2.82 & 106.7 & 29.6 & 16.23 & & 0. \\
\hline 76 & $38.30 \mathrm{~N}$ & $89.00 \mathrm{~W}$ & 2.50 & 14 & 122. & 4.64 & 3.75 & 134.9 & 54.7 & 24.46 & & 0. \\
\hline 77 & $38.50 \mathrm{~N}$ & $80.00 \mathrm{~W}$ & 2.50 & 15 & 237. & 14.38 & 4.47 & 162.1 & 201.9 & 16.04 & & 1. \\
\hline 78 & $38.50 \mathrm{~N}$ & $80.50 \mathrm{~W}$ & 2.50 & 14 & 252. & 19.91 & 4.69 & 151.9 & 293.4 & 27.30 & & 2. \\
\hline 79 & $38.50 \mathrm{~N}$ & $81.00 \mathrm{~W}$ & 2.50 & 13 & 257 & 27.57 & 5.81 & 143.1 & 503.5 & 40.92 & & 2 \\
\hline 80 & $38.50 \mathrm{~N}$ & $91.50 \mathrm{~W}$ & 2.50 & 12 & 267. & 25.30 & 8.07 & 133.2 & 641.4 & 70.06 & & 1. \\
\hline 81 & $38.59 \mathrm{~N}$ & $82.00 \mathrm{w}$ & 2.50 & 11 & 223 & 18.18 & 6.69 & 161.2 & 382.0 & 66.05 & & 0. \\
\hline 82 & $38.50 \mathrm{~N}$ & $82.50 \mathrm{~W}$ & 2.50 & 10 & 150. & 9.15 & 6.37 & 80.5 & 183.2 & 56.36 & & 0. \\
\hline 83 & $38.50 \mathrm{~N}$ & 83.00W & 2.50 & 10 & 155. & 26.38 & 4.83 & 72.6 & 400.4 & 39.86 & & 0. \\
\hline 84 & $38.50 \mathrm{Al}$ & $83.50 \mathrm{~W}$ & 0.88 & 0 & 0. & 0.0 & 0.0 & 0.0 & 0.0 & 0.0 & & 0. \\
\hline 85 & $38.50 \mathrm{~N}$ & $84.00 \mathrm{~W}$ & 0.79 & 0 & 0. & 0.0 & 0.0 & 0.0 & 0.0 & 0.0 & & 0. \\
\hline 86 & $38.53 \mathrm{~N}$ & $84.50 \mathrm{~W}$ & 0.74 & 0 & 0. & 0.0 & 0.0 & 0.0 & 0.0 & 0.0 & & 0. \\
\hline 87 & $38.50 \mathrm{~N}$ & $85.00 \mathrm{~W}$ & 0.69 & 0 & 0. & 0.0 & 0.0 & 0.0 & 0.0 & 0.0 & & 0. \\
\hline 88 & $33.50 N$ & $85.50 \mathrm{~W}$ & 0.62 & 0 & 0. & 0.0 & 0.0 & 0.0 & 0.0 & 0.0 & & 0. \\
\hline 89 & $33.50 \mathrm{~N}$ & $86.00 \mathrm{w}$ & 0.65 & 0 & 0. & 0.0 & 0.0 & 0.0 & 0.0 & 0.0 & & 0. \\
\hline 90 & $38.50 \mathrm{~N}$ & $86.50 \mathrm{~W}$ & 0.79 & 0 & 0. & 0.0 & 0.0 & 0.0 & 0.0 & 0.0 & & 0. \\
\hline 91 & $38.50 \mathrm{~N}$ & $87.00 w$ & 2.50 & 10 & 113. & 10.16 & 5.48 & 143.1 & 174.9 & 29.89 & & 0. \\
\hline 92 & $38.50 \mathrm{~N}$ & $87.50 \mathrm{~W}$ & 2.50 & 11 & 154. & 5.99 & 3.98 & 79.7 & 74.8 & 15.31 & & 0. \\
\hline 93 & $38.50 \mathrm{~N}$ & $88.00 w$ & 2.50 & 12 & $157^{\circ}$ & 5.21 & 2.79 & 3.1 & 45.7 & 10.66 & & 0. \\
\hline 94 & $38.50 \mathrm{~N}$ & $88.50 \mathrm{~W}$ & 2.50 & 12 & 166. & 9.03 & 4.04 & 148.4 & 114.6 & 16.88 & & 0. \\
\hline 95 & $38.50 \mathrm{~N}$ & $89.00 \mathrm{~W}$ & 2.50 & 12 & 169. & 8.49 & 3.82 & 18.4 & 101.8 & 31.93 & & 0. \\
\hline 96 & $39.00 \mathrm{~N}$ & $90.00 W$ & 2.50 & 13 & 277 & 21.61 & 9.10 & 163.0 & 617.8 & 46.18 & & 1. \\
\hline 97 & $39.0 D N$ & $80.50 \mathrm{~W}$ & 2.50 & 12 & 276 & 27.60 & 9.57 & 168.1 & 830.1 & 60.90 & & 1. \\
\hline 98 & $39.00 \mathrm{~N}$ & 81. $00 \mathrm{~W}$ & 2.50 & 11 & 275 & 46.76 & 10.15 & 158.4 & 1491.0 & 80.34 & & 1. \\
\hline 99 & $39.23 N$ & $81.50 \mathrm{~W}$ & 2.50 & 10 & 276. & 42.35 & 11.83 & $14 B .2$ & 1573.5 & 181.19 & & 0. \\
\hline 100 & $39.00 \mathrm{~N}$ & $82.00 \mathrm{~W}$ & 0.90 & 0 & 0. & 0.0 & 0.0 & 0.0 & 0.0 & 0.0 & & 0. \\
\hline 101 & $39.00 \mathrm{~N}$ & 8 2. $50 \mathrm{~W}$ & 0.87 & 0 & 0. & 0.0 & 0.0 & 0.0 & 0.0 & 0.0 & & 0. \\
\hline 102 & $39.00 \mathrm{~N}$ & $83.00 \mathrm{~W}$ & 0.86 & 0 & 0. & 0.0 & 0.0 & 0.0 & 0.0 & 0.0 & & 0. \\
\hline 103 & $39.00 \mathrm{~N}$ & $83.50 \mathrm{~W}$ & 0.88 & 0 & 0. & 0.0 & 0.0 & 0.0 & 0.0 & 0.0 & & 0. \\
\hline 104 & $39.00 \mathrm{~N}$ & 84. $00 \mathrm{~W}$ & 0.88 & 0 & 0. & 0.0 & 0.0 & 0.0 & 0.0 & 0.0 & & \\
\hline 105 & $39.0 \mathrm{~N}$ & $84.50 w$ & 0.87 & 0 & 0 & 0.0 & 0.0 & 0.0 & 0.0 & 0.0 & & 0. \\
\hline 106 & $39.00 \mathrm{~N}$ & 85. OOW & 0.84 & 0 & 0. & 0.0 & 0.0 & 0.0 & 0.0 & 0.0 & & 0. \\
\hline 107 & $39.03 \mathrm{~N}$ & $85.50 \mathrm{~W}$ & 0.74 & 0 & $n$. & 0.0 & 0.0 & 0.0 & 0.0 & 0.0 & & 0. \\
\hline 108 & $39.00 \mathrm{~N}$ & R6. OOW & 0.68 & 0 & 0. & 0.0 & 0.0 & 0.0 & 0.0 & 0.0 & & 0. \\
\hline
\end{tabular}




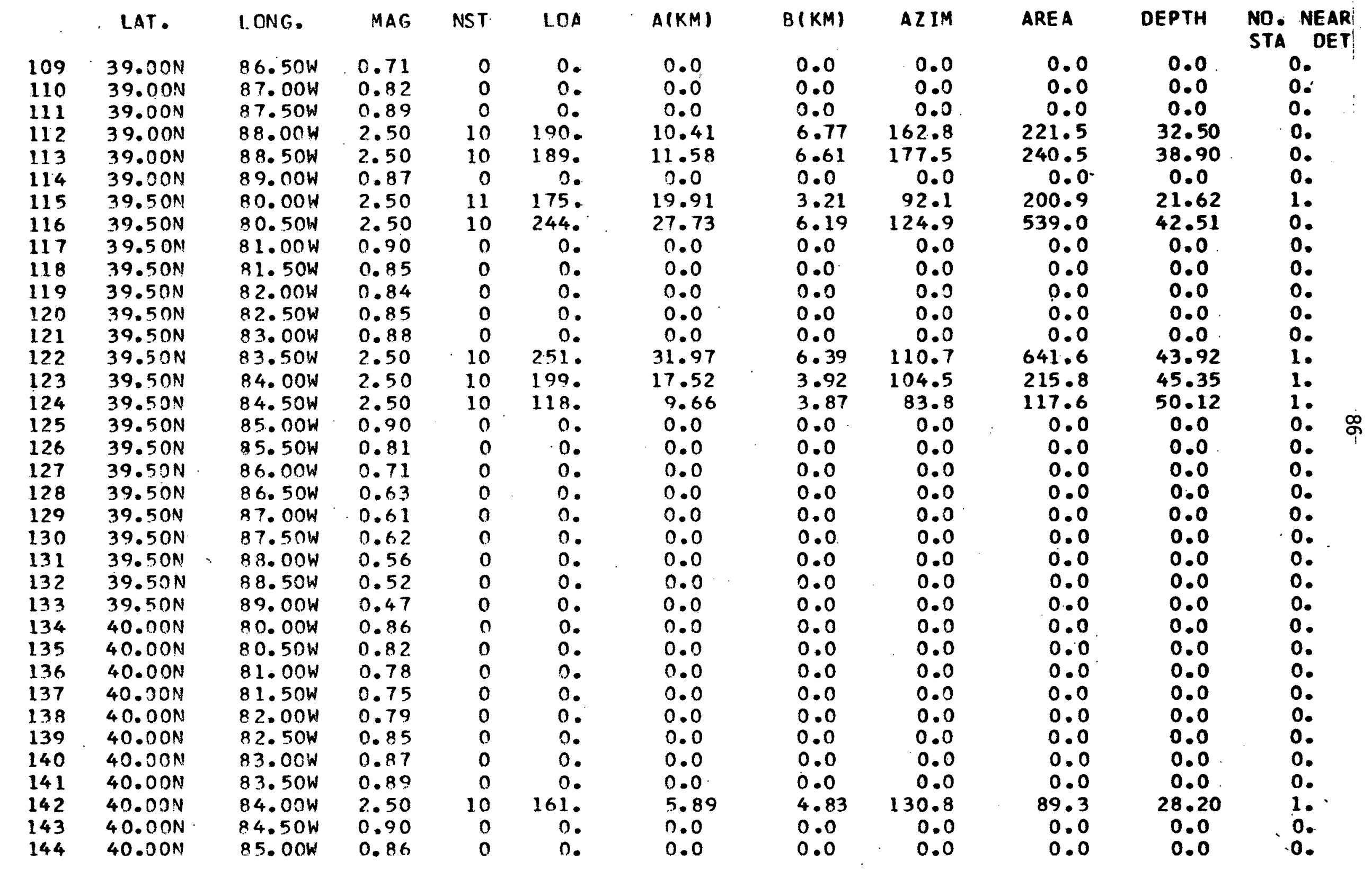




\begin{tabular}{|c|c|c|c|c|c|c|c|c|c|c|c|}
\hline & LAT. & LONG. & MAG & NST & $10 A$ & $A(K M)$ & $B(\mathrm{KM})$ & AZIM & AREA & DEPTH & $\begin{array}{l}\text { NO. NEAR } \\
\text { STA DET }\end{array}$ \\
\hline 145 & $\begin{array}{l}40.00 \mathrm{~N} \\
40.00 \mathrm{~N} .\end{array}$ & $\begin{array}{l}85.50 \mathrm{~W} \\
86.00 \mathrm{~W}\end{array}$ & $\begin{array}{l}0.81 \\
0.72\end{array}$ & & 0. & 0.0 & 0.0 & 0.0 & 0.0 & 0.0 & 0. \\
\hline $\begin{array}{l}146 \\
147\end{array}$ & $\begin{array}{l}40.00 \mathrm{~N} \\
40.00 \mathrm{~N}\end{array}$ & $\begin{array}{l}86.00 \mathrm{~W} \\
86.50 \mathrm{~W}\end{array}$ & $\begin{array}{l}0.72 \\
0.57\end{array}$ & $\begin{array}{l}0 \\
0\end{array}$ & $\begin{array}{l}0 . \\
0 .\end{array}$ & $\begin{array}{l}0.0 \\
0.0\end{array}$ & $\begin{array}{l}0.0 \\
0.0\end{array}$ & $\begin{array}{l}0.0 \\
0.0\end{array}$ & $\begin{array}{l}0.0 \\
0.0\end{array}$ & $\begin{array}{l}0.0 \\
0.0\end{array}$ & $\begin{array}{l}0 . \\
0 .\end{array}$ \\
\hline 148 & $40.00 \mathrm{~N}$ & $87.00 \mathrm{~W}$ & $0 . .44$ & 0 & 0. & 0.0 & 0.0 & 0.0 & 0.0 & 0.0 & 0. \\
\hline 149 & $40.00 \mathrm{~N}$ & $87.50 \mathrm{~W}$ & $0.3: 4$ & 0 & 0. & 0.0 & 0.0 & 0.0 & 0.0 & 0.0 & 0. \\
\hline 150 & $40.00 \mathrm{~N}$ & $88.00 \mathrm{~W}$ & 0.26 & 0 & 0. & 0.0 & 0.0 & 0.0 & 0.0 & 0.0 & 0. \\
\hline 151 & $40.00 \mathrm{~N}$ & $8.8 .50 \mathrm{~W}$ & 0.18 & 0 & 0. & 0.0 & 0.0 & 0.0 & 0.0 & 0.0 & 0. \\
\hline 152 & $40.00 \mathrm{~N}$ & $89.00 \mathrm{~W}$ & 0.13 & 0 & 0. & 0.0 & 0.0 & 0.0 & 0.0 & 0.0 & 0. \\
\hline 153 & $40.5 \mathrm{ON}$ & $80.00 \mathrm{~W}$ & 0.59 & 0 & 0. & 0.0 & 0.0 & 0.0 & 0.0 & 0.0 & 0. \\
\hline 154 & $40.50 \mathrm{~N}$ & $80.50 \mathrm{~W}$ & 0.61 & 0 & 0. & 0.0 & 0.0 & 0.0 & 0.0 & 0.0 & 0. \\
\hline 155 & $40.50 \mathrm{~N}$ & $81.00 \mathrm{w}$ & 0.66 & 0 & 0. & 0.0 & 0.0 & 0.0 & 0.0 & 0.0 & 0. \\
\hline 156 & $40.50 \mathrm{~N}$ & $81.50 \mathrm{~W}$ & 0.69 & 0 & 0. & 0.0 & 0.0 . & 0.0 & 0.0 & 0.0 & 0. \\
\hline 157 & $40.50 \mathrm{~N}$ & $82.00 \mathrm{~W}$ & 0.75 & 0 & 0. & 0.0 & 0.0 & 0.0 & 0.0 & 0.0 & 0. \\
\hline 158 & $40.50 \mathrm{~N}$ & $82.50 \mathrm{~W}$ & 0.84 & 0. & 0 & 0.0 & 0.0 & 0.0 & 0.0 & 0.0 & 0. \\
\hline 159 & $40.50 \mathrm{~N}$ & $83.00 \mathrm{~W}$ & 0.88 & 0 & 0. & 0.0 & 0.0 & 0.0 & 0.0 & 0.0 & 0. \\
\hline 160 & $40.50 \mathrm{~N}$ & $83.50 \mathrm{~W}$ & 0.89 & 0 & 0. & 0.0 & 0.0 & 0.0 & 0.0 & 0.0 & 0. \\
\hline 161 & $40.50 \mathrm{~N}$ & 84. OOW & 0.88 & 0 & 0. & 0.0 & 0.0 & 0.0 & 0.0 & 0.0 & 0. \\
\hline 162 & $40.50 \mathrm{~N}$ & $84.50 \mathrm{~W}$ & 0.84 & 0 & 0. & 0.0 & 0.0 & 0.0 & 0.0 & 0.0 & 0. \\
\hline 163 & $40.50 \mathrm{~N}$ & $85.00 \mathrm{~W}$ & 0.81 & 0 & 0. & 0.0 & 0.0 & 0.0 & 0.0 & 0.0 & 0. \\
\hline 164 & $40.50 \mathrm{~N}$ & $85.50 \mathrm{~W}$ & 0.76 & 0 & 0. & 0.0 & 0.0 & 0.0 & 0.0 & 0.0 & 0. \\
\hline 165 & $40.50 \mathrm{~N}$ & $86.00 \mathrm{~W}$ & 0.65 & 0 & 0. & 0.0 & 0.0 & 0.0 & 0.0 & 0.0 & 0. \\
\hline 166 & $40.50 \mathrm{~N}$ & $86.50 \mathrm{~W}$ & 0.47 & 0 & 0. & 0.0 & 0.0 & 0.0 & 0.0 & 0.0 & 0. \\
\hline 167 & $40.50 \mathrm{~N}$ & $87.00 \mathrm{~W}$ & 0.30 & 0 & 0. & 0.0 & 0.0 & 0.0 & 0.0 & 0.0 & 0. \\
\hline 168 & $40.50 \mathrm{~N}$ & $87.50 \mathrm{~W}$ & 0.19 & 0 & 0. & 0.0 & 0.0 & 0.0 & 0.0 & 0.0 & 0. \\
\hline 169 & $40.50 \mathrm{~N}$ & $88.00 W$ & 0.10 & 0 & 0. & 0.0 & 0.0 & 0.0 & 0.0 & 0.0 & 0. \\
\hline 170 & $40.50 \mathrm{~N}$ & $88.50 \mathrm{~W}$ & 0.06 & 0 & 0. & 0.0 & 0.0 & 0.0 & 0.0 & 0.0 & 0. \\
\hline 171 & $40.50 \mathrm{~N}$ & $89.00 \mathrm{~W}$ & 0.03 & 0 & 0. & 0.0 & 0.0 & 0.0 & 0.0 & 0.0 & 0. \\
\hline 172 & $41.00 \mathrm{~N}$ & $80.00 \mathrm{~W}$ & 0.42 & 0 & 0. & 0.0 & 0.0 & 0.0 & 0.0 & 0.0 & 0. \\
\hline 173 & $41.30 \mathrm{~N}$ & $80.50 \mathrm{~W}$ & 0.48 & 0 & 0 & 0.0 & 0.0 & 0.0 & 0.0 & 0.0 & 0. \\
\hline 174 & $41.00 \mathrm{~N}$ & $81.00 \mathrm{~W}$ & 0.55 & 0 & 0 & 0.0 & 0.0 & 0.0 & 0.0 & 0.0 & 0. \\
\hline 175 & $41.00 \mathrm{~N}$ & $81.50 \mathrm{H}$ & 0.62 & 0 & 0. & 0.0 & 0.0 & 0.0 & 0.0 & 0.0 & 0. \\
\hline 176 & $41.00 N$ & $82.00 \mathrm{~W}$ & 0.70 & 0 & 0. & 0.0 & 0.0 & 0.0 & 0.0 & 0.0 & 0. \\
\hline 177. & $41.00 \mathrm{~N}$ & $82.50 \mathrm{~W}$ & 0.84 & 0 & 0. & 0.0 & 0.0 & 0.0 & 0.0 & 0.0 & 0. \\
\hline 178 & $41.00 \mathrm{~N}$ & $83.00 \mathrm{~W}$ & 2.50 & 10 & 271 & 31.01 & 7.69 & 86.1 & 749.12 & 45.63 & 1. \\
\hline 179 & $41.00 \mathrm{~N}$ & $83.50 \mathrm{~W}$ & 2.50 & 10 & 202 & 9.94 & 3.68 & 104.4 & 114.9 & 42.81 & 1.1 \\
\hline 180 & $41.00 \mathrm{~N}$ & 84. OOW & 2.50 & 10 & 146 & 5.03 & 3.03 & 126.6 & 47.8 & 26.26 & 1. \\
\hline
\end{tabular}




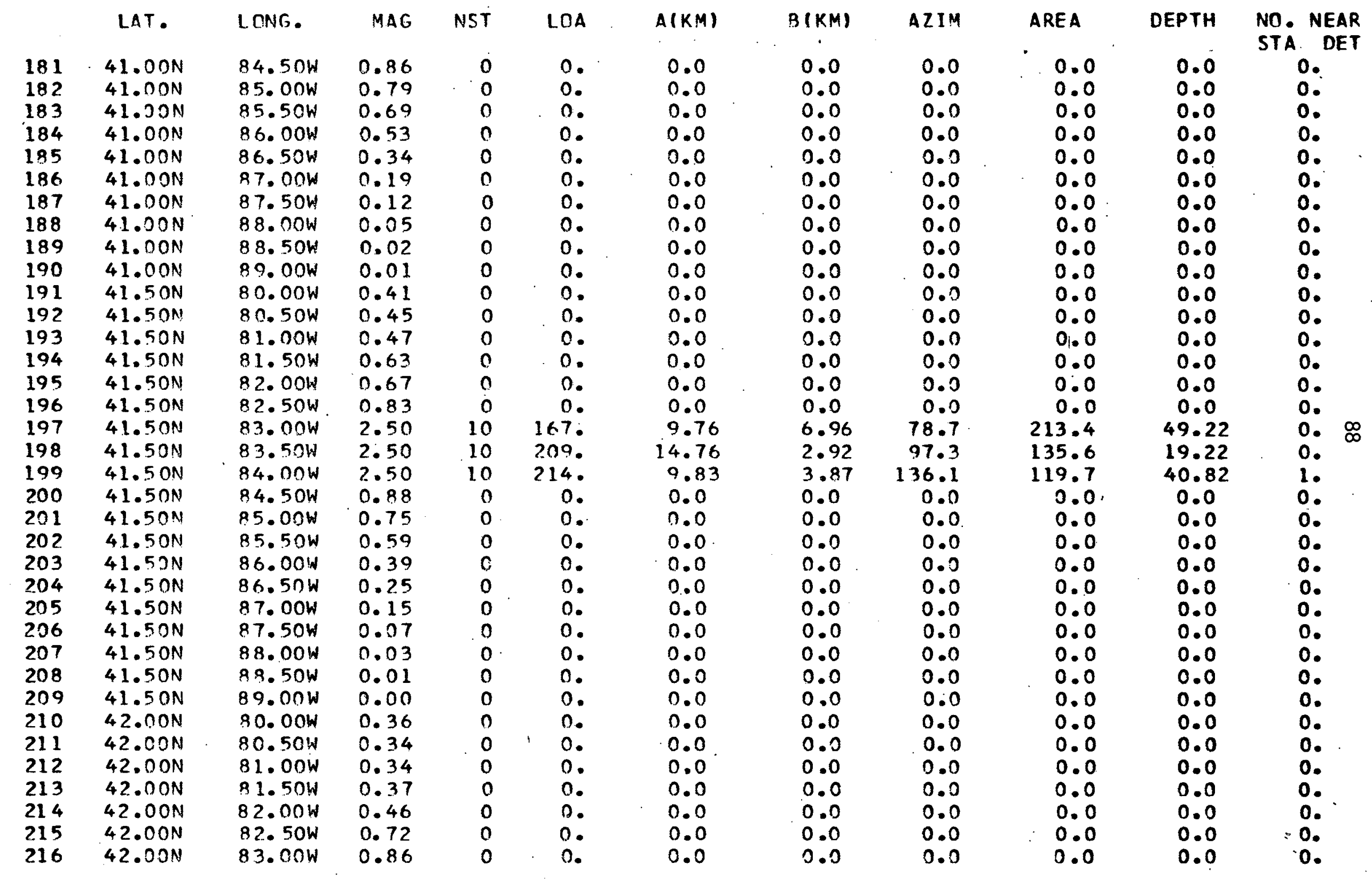




\begin{tabular}{|c|c|c|c|c|c|c|c|c|c|c|c|}
\hline & LAT. & LONG. & MAG & NST & LOA & $A(K M)$ & $B(K M)$ & AZIM & AREA & DEPTH & $\begin{array}{l}\text { NO: NEAR } \\
\text { STA DET }\end{array}$ \\
\hline $\begin{array}{l}217 \\
218\end{array}$ & $\begin{array}{l}42.00 \mathrm{~N} \\
42.00 \mathrm{~N}\end{array}$ & $\begin{array}{l}83.50 \mathrm{~W} \\
84.00 \mathrm{~W}\end{array}$ & $\begin{array}{l}0.90 \\
0.89\end{array}$ & $\begin{array}{l}0 \\
0\end{array}$ & O. & $\begin{array}{l}0.0 \\
0.0\end{array}$ & $\begin{array}{l}0.0 \\
0.0\end{array}$ & $\begin{array}{l}0.0 \\
0.0\end{array}$ & $\begin{array}{l}0.0 \\
0.0\end{array}$ & $\begin{array}{l}0.0 \\
0.0\end{array}$ & $\begin{array}{l}0 . \\
0 .\end{array}$ \\
\hline 219 & $42.00 N !$ & $84.50 \mathrm{~W}$ & 0.83 & 0 & 0. & 0.0 & 0.0 & 0.0 & 0.0 & 0.0 & 0. \\
\hline 220 & $42.00 \mathrm{~N}$ & $85.00 \mathrm{~W}$ & 0.62 & 0 & 0. & 0.0 & 0.0 & 0.0 & 0.0 & 0.0 & 0. \\
\hline 221 & $42.00 \mathrm{~N}$ & $85.50 \mathrm{~W}$ & 0.41 & 0 & 0. & 0.0 & 0.0 & 0.0 & 0.0 & 0.0 & 0. \\
\hline 222 & $42.00 \mathrm{~N}$ & $86.00 \mathrm{~W}$ & 0.24 & 0 & 0. & 0.0 & 0.0 & 0.0 & 0.0 & 0.0 & 0. \\
\hline 223 & $42.00 \mathrm{~N}$ & $86.50 \mathrm{~W}$ & 0.18 & 0 & 0. & 0.0 & 0.0 & 0.0 & 0.0 & 0.0 & 0. \\
\hline 224 & $42.00 \mathrm{~N}$ & $87.00 \mathrm{~W}$ & 0.09 & 0 & 0. & 0.0 & 0.0 & 0.0 & 0.0 & 0.0 & 0. \\
\hline 226 & $42.00 N$ & $87.50 \mathrm{~W}$ & 0.03 & 0 & 0. & 0.0 & 0.0 & 0.0 & 0.0 & 0.0 & o. \\
\hline 226 & $42.00 \mathrm{~N}$ & $88.00 \mathrm{~W}$ & 0.01 & 0 & 0. & 0.0 & 0.0 & 0.0 & 0.0 & 0.0 & 0. \\
\hline 227 & $42.00 \mathrm{~N}$ & $88.50 \mathrm{~W}$ & 0.00 & 0 & 0. & 0.0 & 0.0 . & 0.0 & 0.0 & 0.0 & 0. \\
\hline 228 & $42.00 \mathrm{~N}$ & $89.00 \mathrm{~W}$ & 0.00 & 0 & 0. & 0.0 & 0.0 & 0.0 & 0.0 & 0.0 & - \\
\hline
\end{tabular}


NETWORK SUMMARY- ERROR ELLIPSES

(MAGNITUDES RANGE FROM, 2.50 TO 2.50 IN STEPS OF 0.0 )

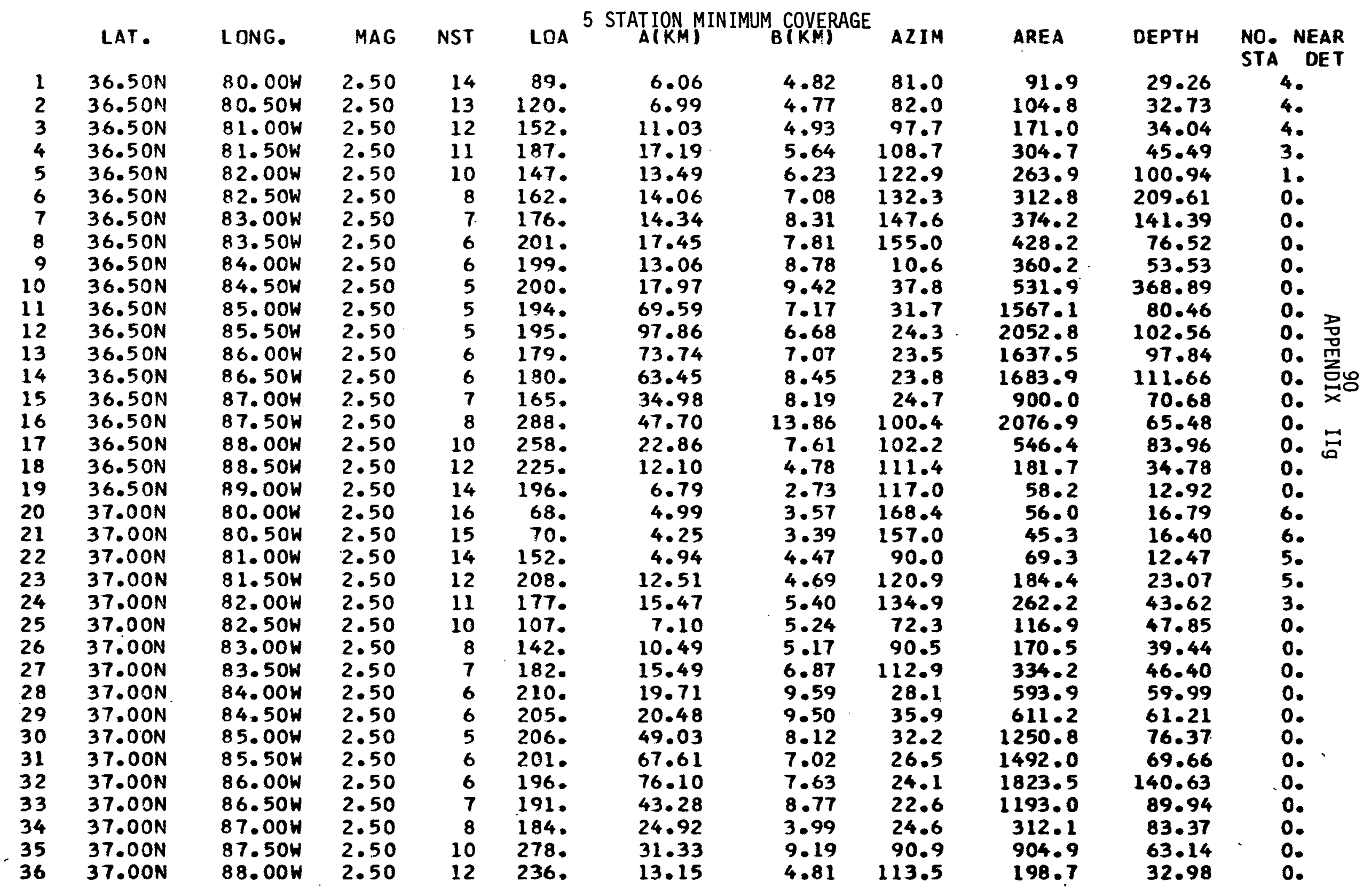




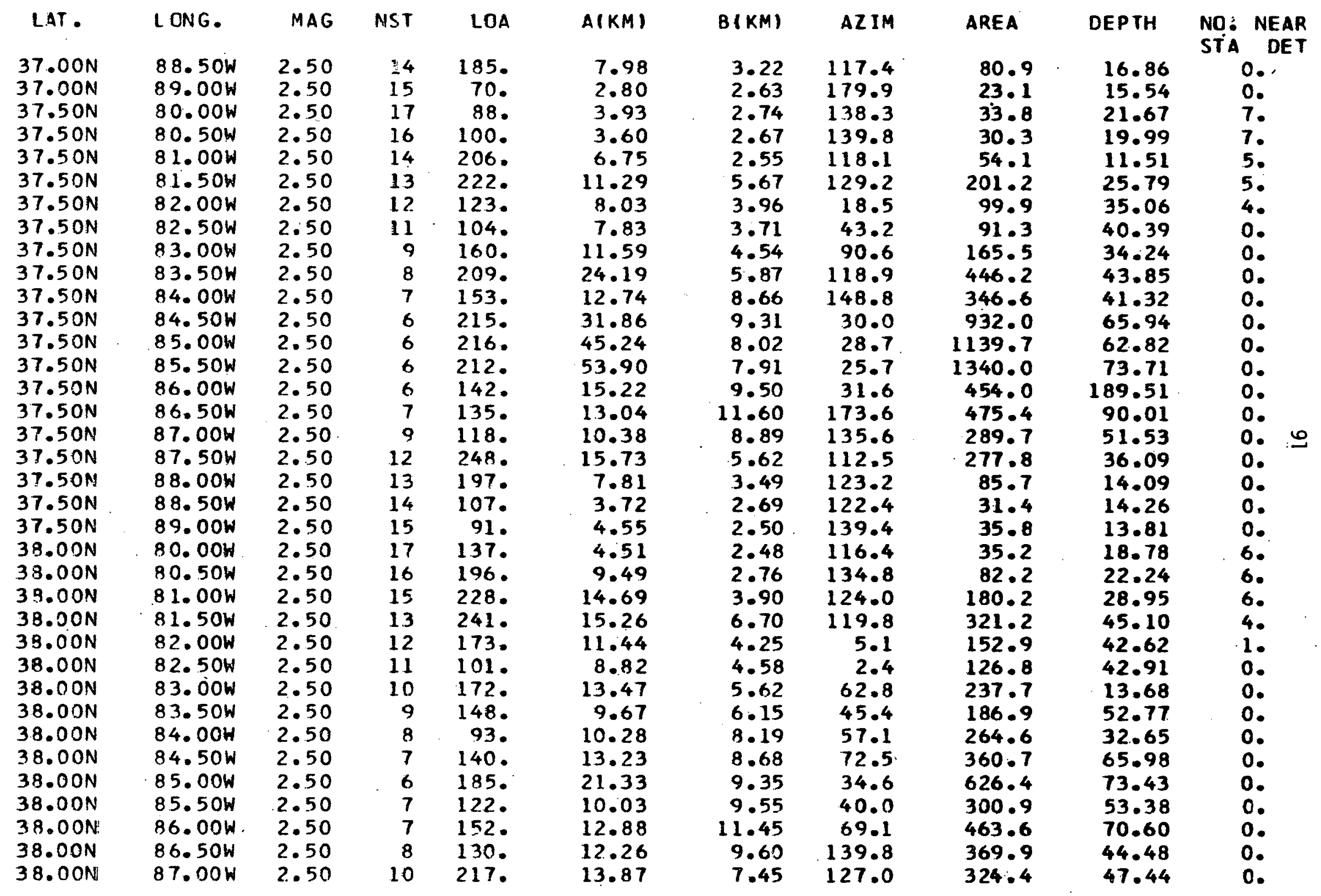




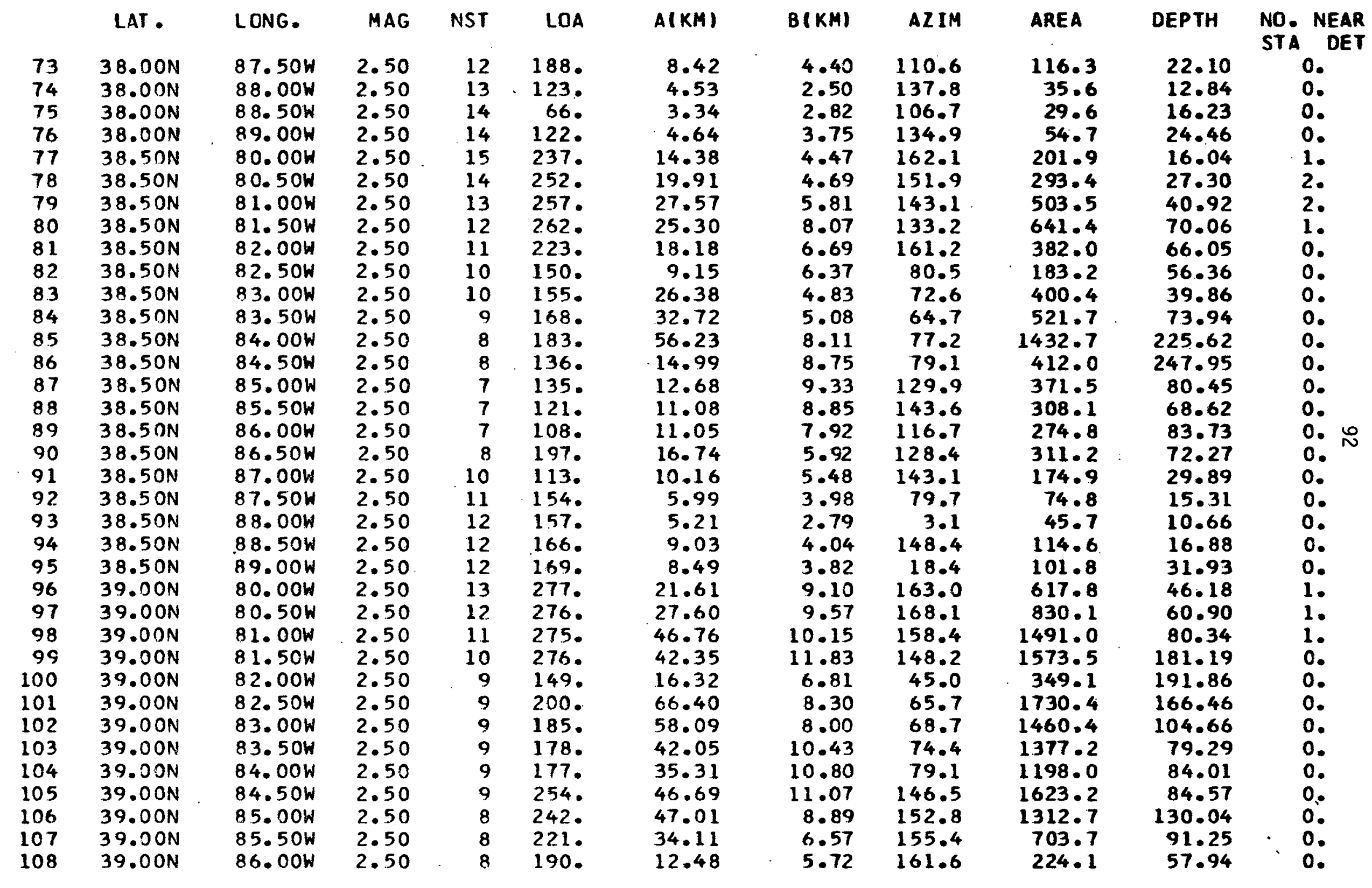




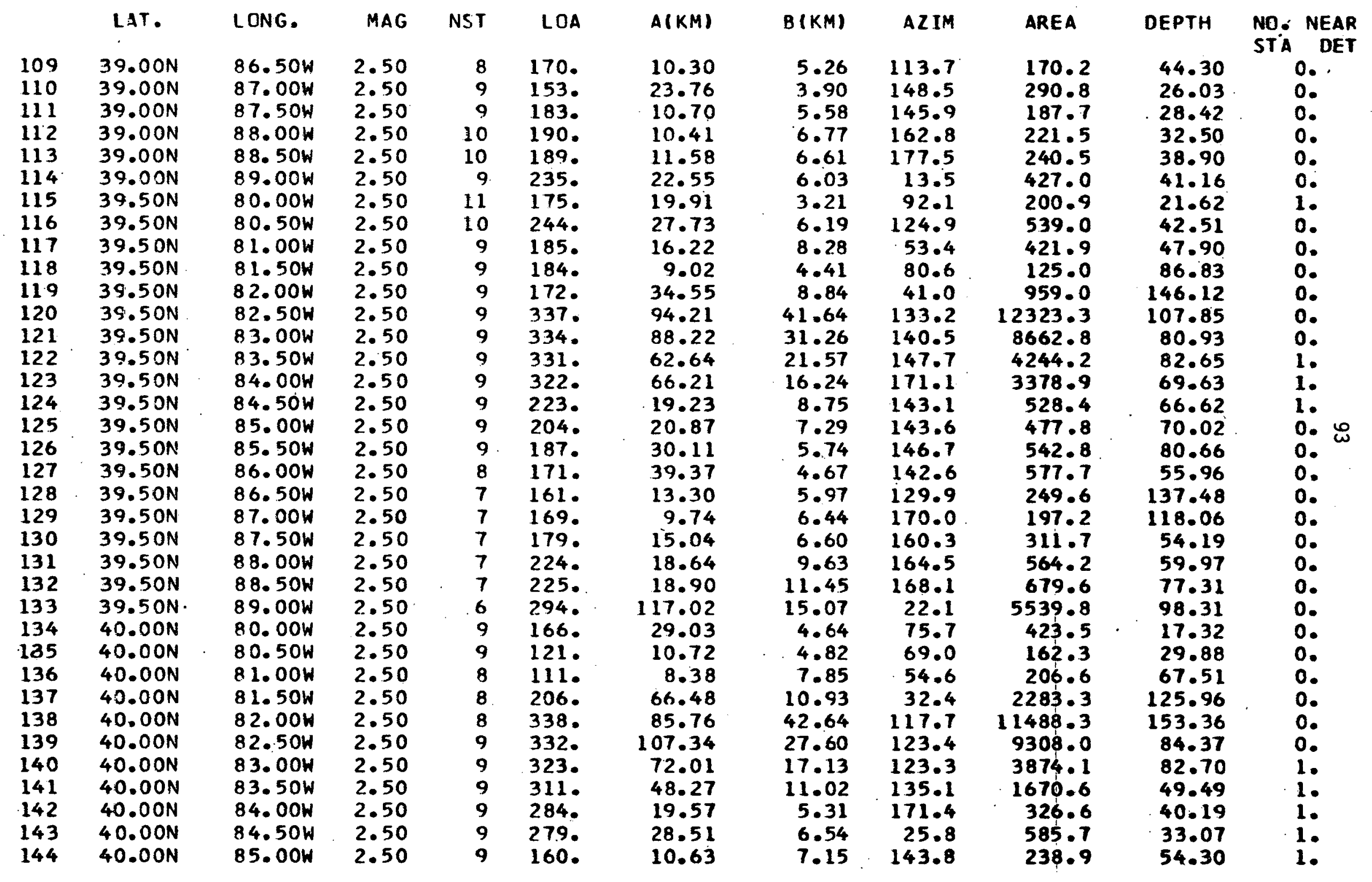




\begin{tabular}{|c|c|c|c|c|c|c|c|c|c|c|c|c|}
\hline & LAT . & LONG. & MAG & NST & LOA & $A(K M)$ & $B(K M)$ & AZIM & AREA & DEPTH & NO. & $\begin{array}{r}\text { NEAR } \\
\text { DET }\end{array}$ \\
\hline $\begin{array}{l}145 \\
146\end{array}$ & $\begin{array}{l}40.00 \mathrm{~N} \\
40.0 \mathrm{NN}\end{array}$ & $\begin{array}{l}85.50 \mathrm{~W} \\
86.0 \mathrm{WW}\end{array}$ & $\begin{array}{l}2.50 \\
2.50\end{array}$ & $\begin{array}{l}9 \\
8\end{array}$ & $\begin{array}{l}183 . \\
210 .\end{array}$ & $\begin{array}{l}20.48 \\
44.32\end{array}$ & $\begin{array}{l}6.39 \\
6.18\end{array}$ & $\begin{array}{l}140.5 \\
134.4\end{array}$ & $\begin{array}{l}410.8 \\
861.0\end{array}$ & $\begin{array}{l}60.87 \\
79.17\end{array}$ & & $\begin{array}{l}0 . \\
0 .\end{array}$ \\
\hline 147 & $40.00 \mathrm{~N}$ & $86.50 \mathrm{~W}$ & 2.50 & 7 & 181. & 16.76 & 6.53 & 135.8 & 343.8 & 132.45 & & 0. \\
\hline 148 & $40.00 \mathrm{~N}$ & $87.00 \mathrm{~W}$ & 2.50 & 6 & 188. & 13.39 & 7.85 & 147.4 & 329.9 & 437.37 & & 0. \\
\hline 149 & $40.00 \mathrm{~N}$ & $87.50 \mathrm{~W}$ & 2.50 & 6 & 195. & 16.47 & 7.97 & 154.0 & 412.7 & 220.41 & & 0. \\
\hline 150 & $40.00 \mathrm{~N}$ & $88.00 \mathrm{~W}$ & 2.50 & 5 & 204. & 20.90 & 8.89 & 157.8 & 583.8 & 381.37 & & 0. \\
\hline 151 & $40.00 \mathrm{~N}$ & B8.50W & 2.50 & 5 & 250. & 43.06 & 13.64 & 173.9 & 1845.1 & 80.83 & & 0. \\
\hline 152 & $40.00 \mathrm{~N}$ & $89.00 \mathrm{~W}$ & 0.90 & 0 & 0. & 0.0 & 0.0 & 0.0 & 0.0 & 0.0 & & 0. \\
\hline 153 & $40.50 \mathrm{~N}$ & $80.00 \mathrm{~W}$ & 2.50 & 7 & 177. & 19.68 & 6.56 & 61.0 & 405.8 & 30.55 & & 0. \\
\hline 154 & $40.50 \mathrm{~N}$ & $80.50 \mathrm{~W}$ & 2.50 & 7 & 116. & 8.70 & 5.44 & 120.9 & 148.8 & 45.01 & & 0. \\
\hline 155 & $40.5 \mathrm{ON}$ & $81.00 \mathrm{H}$ & 2.50 & 7 & 195. & 2.3 .43 & 5.57 & 167.7 & 410.4 & 89.00 & & 0. \\
\hline 156 & $40.50 \mathrm{~N}$ & $81.50 \mathrm{~W}$ & 2.50 & 7 & 185. & 21.80 & 6.51 & 170.3 & 446.1 & 197.29 & & 0. \\
\hline 157 & $40.50 \mathrm{~N}$ & $82.00 \mathrm{~W}$ & 2.50 & 8 & 334. & 103.80 & 36.40 & 94.4 & 11868.8 & 125.53 & & 0. \\
\hline 158 & $40.50 \mathrm{~N}$ & $82.50 \mathrm{~W}$ & 2.50 & 9 & 326. & 67.93 & 21.37 & 93.3 & 4560.4 & 105.97 & & 0. \\
\hline 159 & $40.50 \mathrm{~N}$ & $83.00 \mathrm{~W}$ & 2.50 & 9 & 310. & 77.26 & 11.64 & 92.9 & 2826.0 & 48.57 & & 1. \\
\hline 160 & $40.50 \mathrm{~N}$ & $83.50 \mathrm{~W}$ & 2.50 & 9 & 261. & 13.54 & 5.34 & 85.9 & 227.1 & 28.19 & & 1. \\
\hline 161 & $40.5 \mathrm{ON}$ & $84.00 \mathrm{~W}$ & 2.50 & 9 & 83. & 3.60 & 2.25 & 162.7 & 25.4 & 22.21 & & 1. \\
\hline 162 & $40.50 \mathrm{~N}$ & $84.50 \mathrm{~W}$ & 2.50 & 9 & 142. & 3.12 & 1.90 & 55.1 & 18.6 & 19.02 & & i. \\
\hline 163 & $40.5 \mathrm{ON}$ & $85.00 \mathrm{~W}$ & 2.50 & 9 & 301. & 40.81 & 8.31 & 86.2 & 1065.4 & 31.26 & & 1. \\
\hline 164 & $40.50 \mathrm{~N}$ & $85.50 \mathrm{~W}$ & 2.50 & 8 & 322. & 77.94 & 18.06 & 90.2 & 4423.1 & 75.74 & & 1. \\
\hline 165 & $40.5 \mathrm{ON}$ & $86.00 \mathrm{~W}$ & 2.50 & 8 & 235. & 42.48 & 8.91 & 141.1 & 1189.4 & 74.51 & & c. \\
\hline 166 & $40.5 \mathrm{ON}$ & $86.50 \mathrm{~W}$ & 2.50 & 7 & 198. & 23.77 & 7.88 & 149.4 & 588.3 & 138.73 & & 0. \\
\hline 167 & $40.50 \mathrm{~N}$ & $87.00 \mathrm{~W}$ & 2.50 & 6 & 204. & 21.38 & 8.42 & 150.5 & 565.2 & 345.88 & & 0 \\
\hline 168 & $40.5 \mathrm{ON}$ & $87.50 \mathrm{~W}$ & 2.50 & 5 & 213. & 22.81 & 9.72 & 155.2 & 696.6 & 279.04 & & 0. \\
\hline 169 & $40.50 \mathrm{~N}$ & $88.00 W$ & 0.87 & 0 & 0. & 0.0 & 0.0 & 0.0 & 0.0 & 0.0 & & 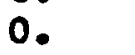 \\
\hline 170 & $40.50 \mathrm{~N}$ & $88.50 \mathrm{~W}$ & 0.79 & 0 & 0. & 0.0 & 0.0 & 0.0 & 0.0 & 0.0 & & 0. \\
\hline 171 & $40.50 \mathrm{~N}$ & $89.00 \mathrm{~W}$ & 0.70 & 0 & 0. & 0.0 & 0.0 & 0.0 & 0.0 & 0.0 & & 0. \\
\hline 172 & $41.00 \mathrm{~N}$ & $80.00 \mathrm{~W}$ & 2.50 & 6 & 185. & 14.66 & 7.47 & 88.9 & 344.2 & 79.86 & & \\
\hline 173 & $41.00 \mathrm{~N}$ & $80.50 \mathrm{~W}$ & 2.50 & 6 & 172. & 13.97 & 6.02 & 58.0 & 264.1 & 130.68 & & 0. \\
\hline 174 & $41.00 \mathrm{~N}$ & $81.00 \mathrm{~W}$ & 2.50 & 7 & 138. & 11.20 & 6.25 & 29.1 & 219.8 & 186.48 & & 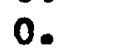 \\
\hline 175 & $41.00 \mathrm{~N}$ & $81.50 \mathrm{~W}$ & 2.50 & 7 & 139. & 16.28 & 6.94 & 2.8 & 355.0 & 183.53 & & 0 \\
\hline 176 & $41.0 \mathrm{ON}$ & $82.00 \mathrm{~W}$ & 2.50 & 8 & 227. & 47.43 & 11.49 & 135.7 & 1712.4 & 110.20 & & 0 \\
\hline 177 & $41.00 \mathrm{~N}$ & $82.50 \mathrm{~W}$ & 2.50 & 9 & 210 & 26.23 & 9.77 & 137.7 & 805.4 & 69.32 & & \\
\hline 178 & $41.00 \mathrm{~N}$ & $83.0 \mathrm{OW}$ & 2.50 & 9 & 313. & 69.73 & 14.70 & 63.7 & 3219.7 & 75.18 & & 1 \\
\hline 179 & $41.00 \mathrm{~N}$ & $83.50 \mathrm{~W}$ & 2.50 & 9 & 202 . & 10.05 & 4.89 & 101.0 & 154.5 & 49.10 & & 1. \\
\hline 180 & $41.00 \mathrm{~N}$ & $84.00 \mathrm{~W}$ & 2.50 & 9 & 146. & 5.09 & 4.66 & 147.0 & 74.6 & 26.91 & & \\
\hline
\end{tabular}




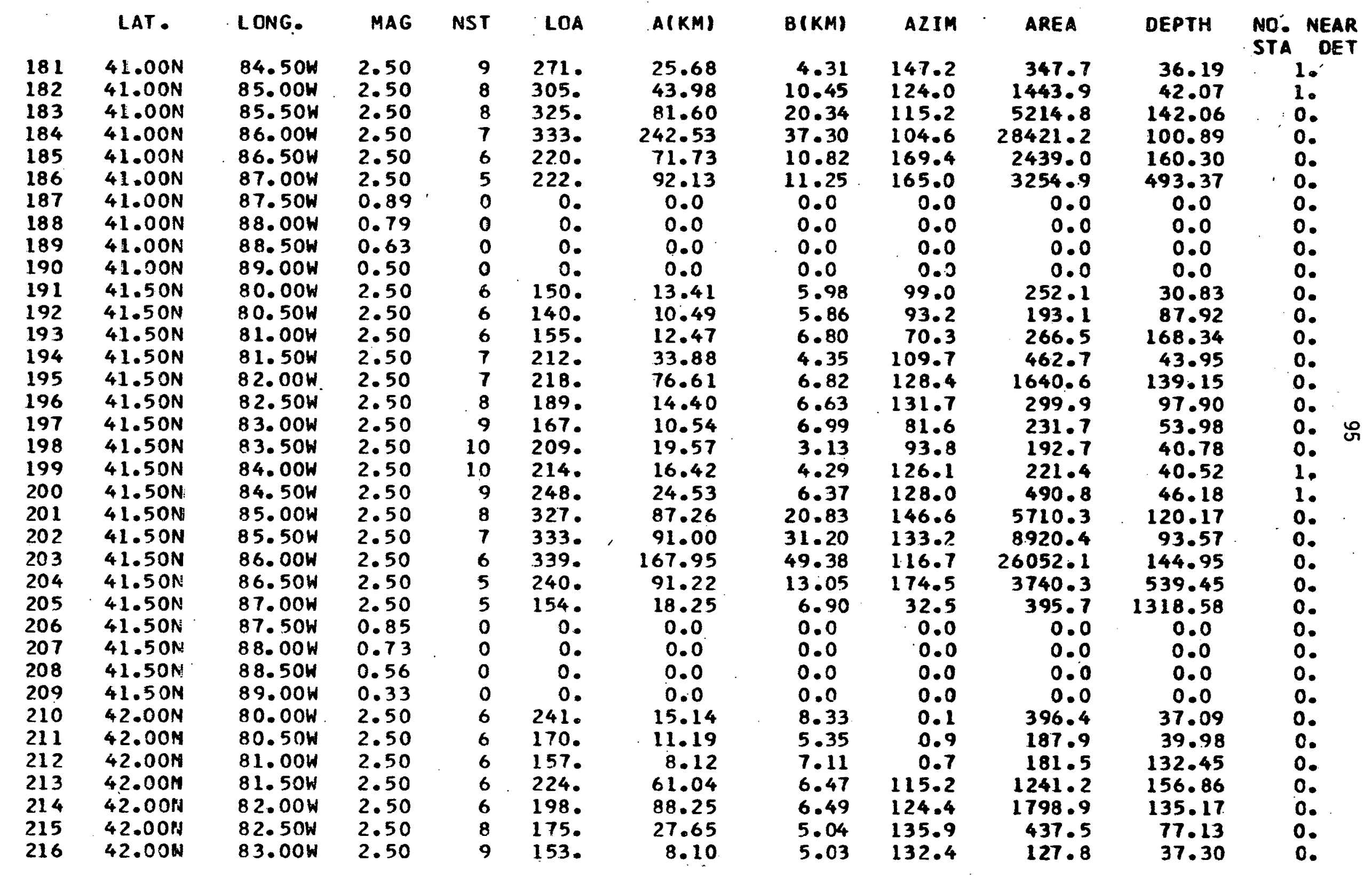




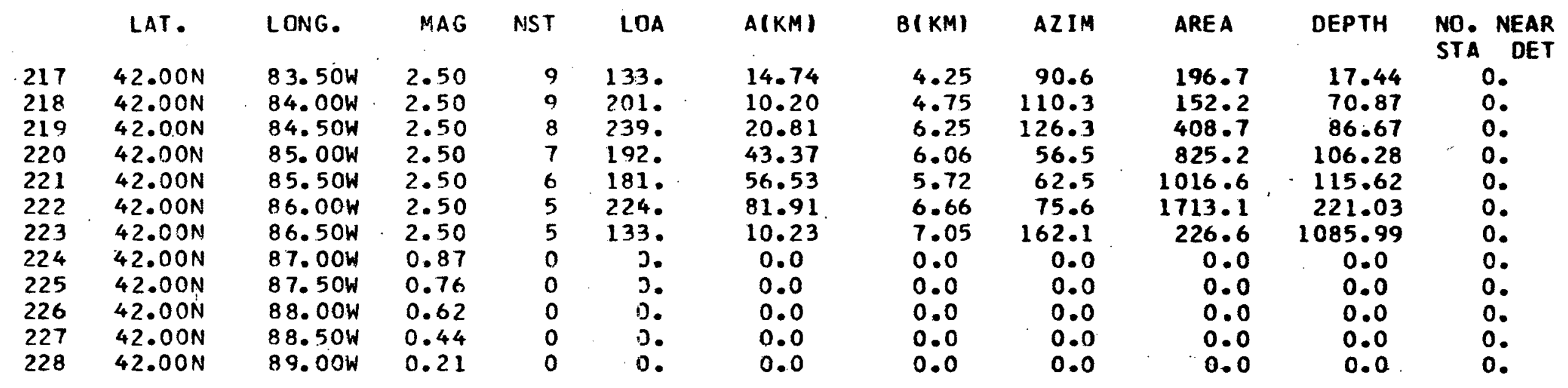


NETWORK SUMMARY - ERROR ELLIPSES

(MAGNITUDES RANGE FROM, 2.00 TO 2.00 IN STEPS OF 0.0 .)

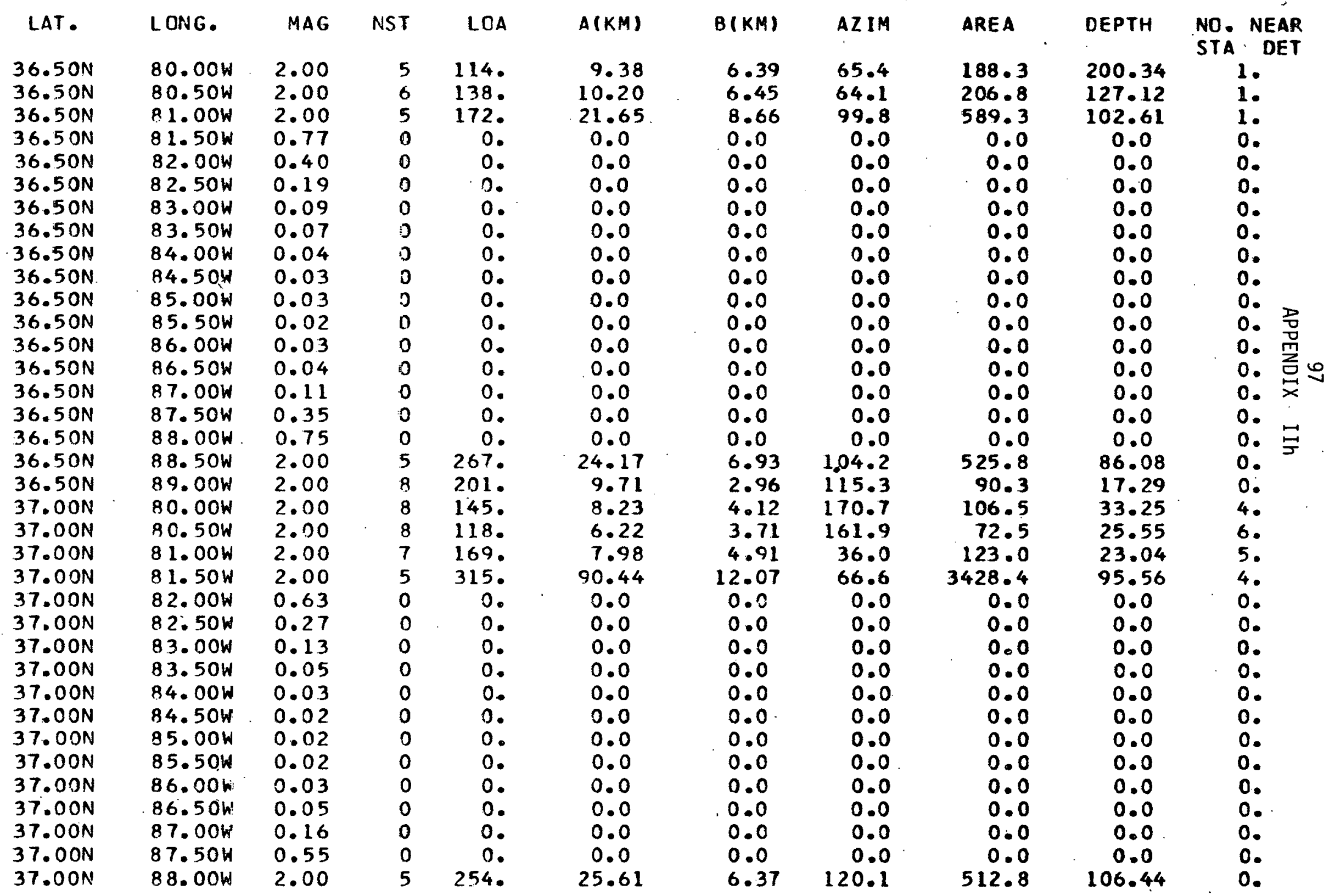




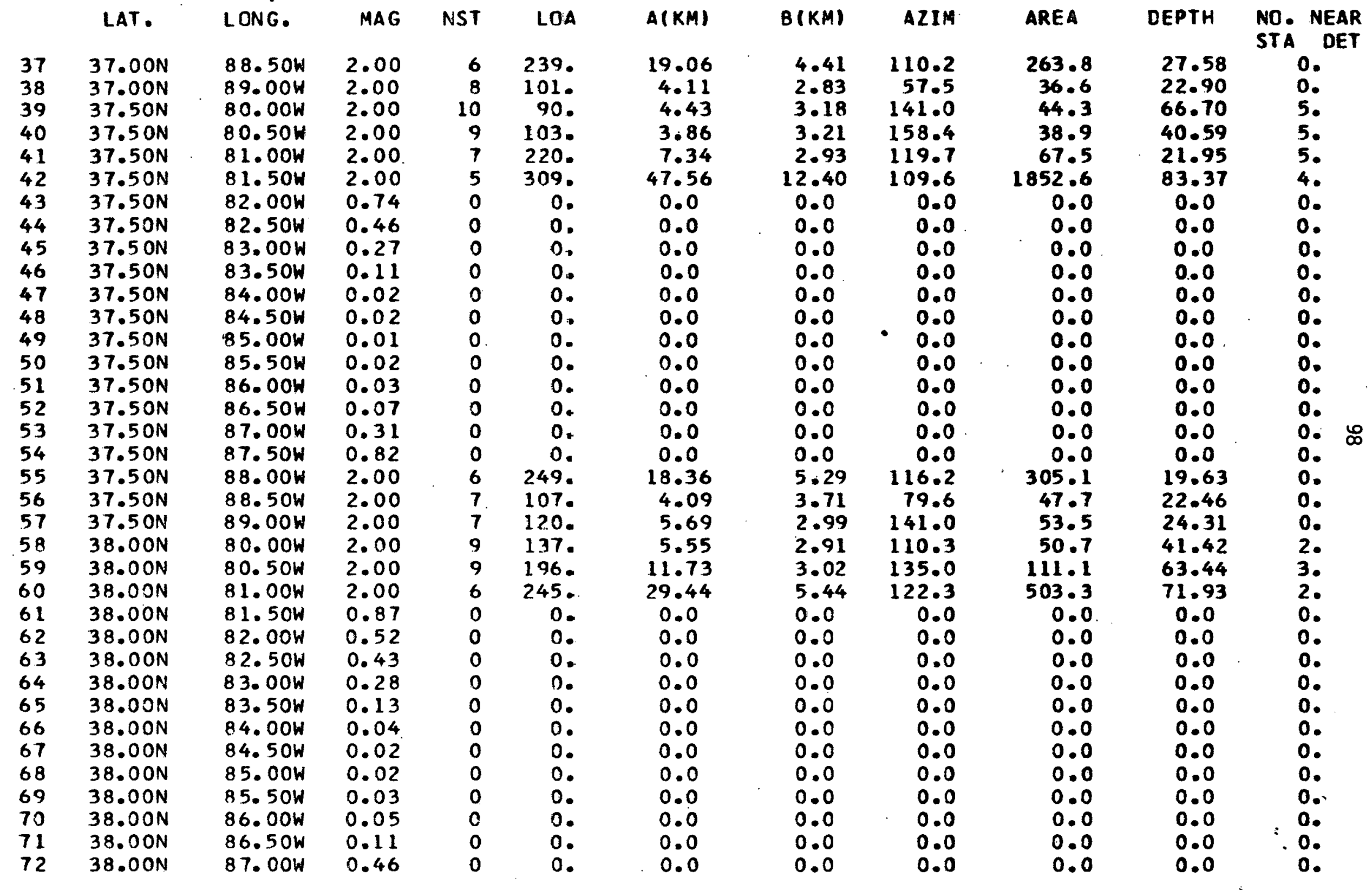




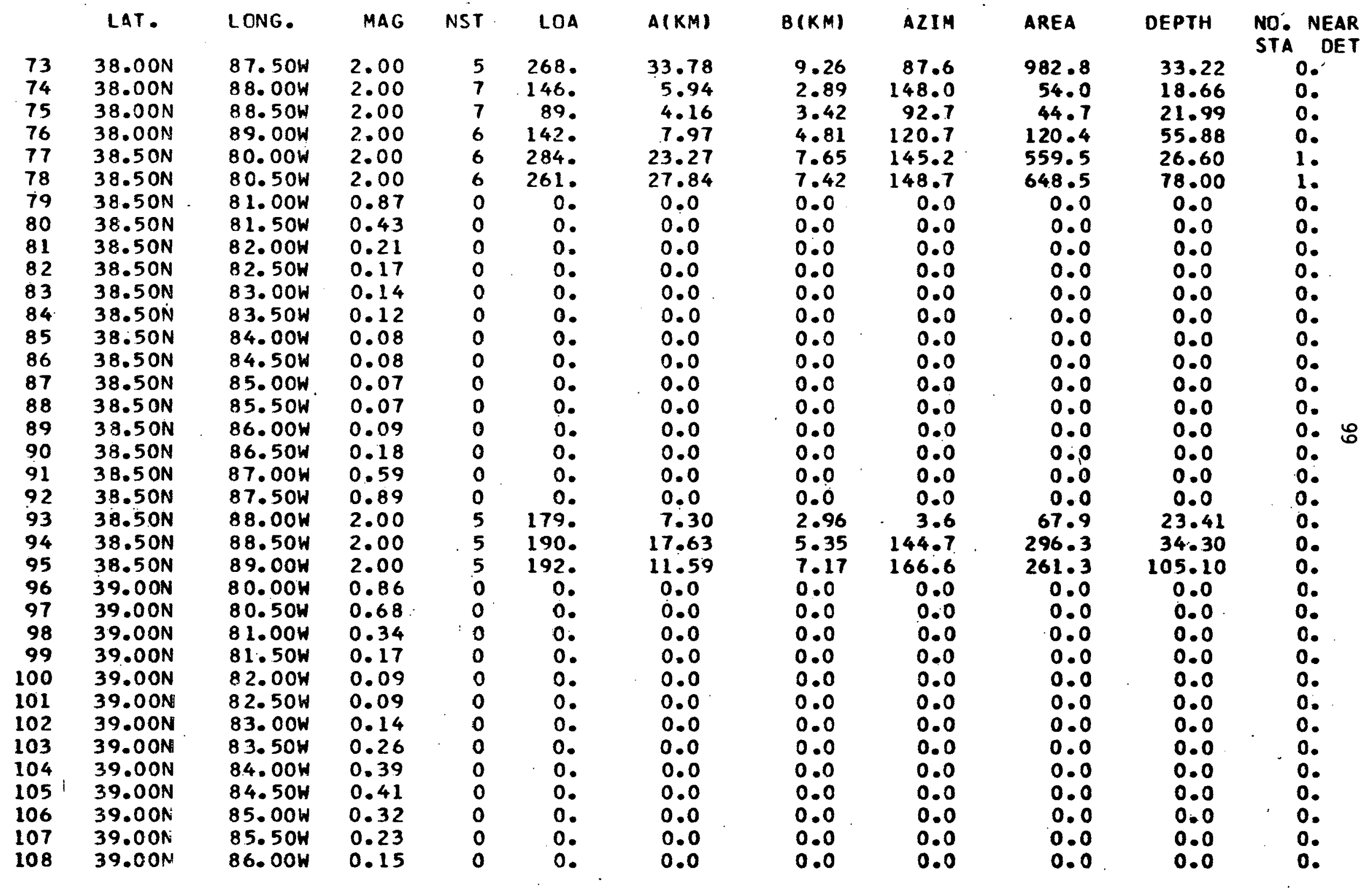




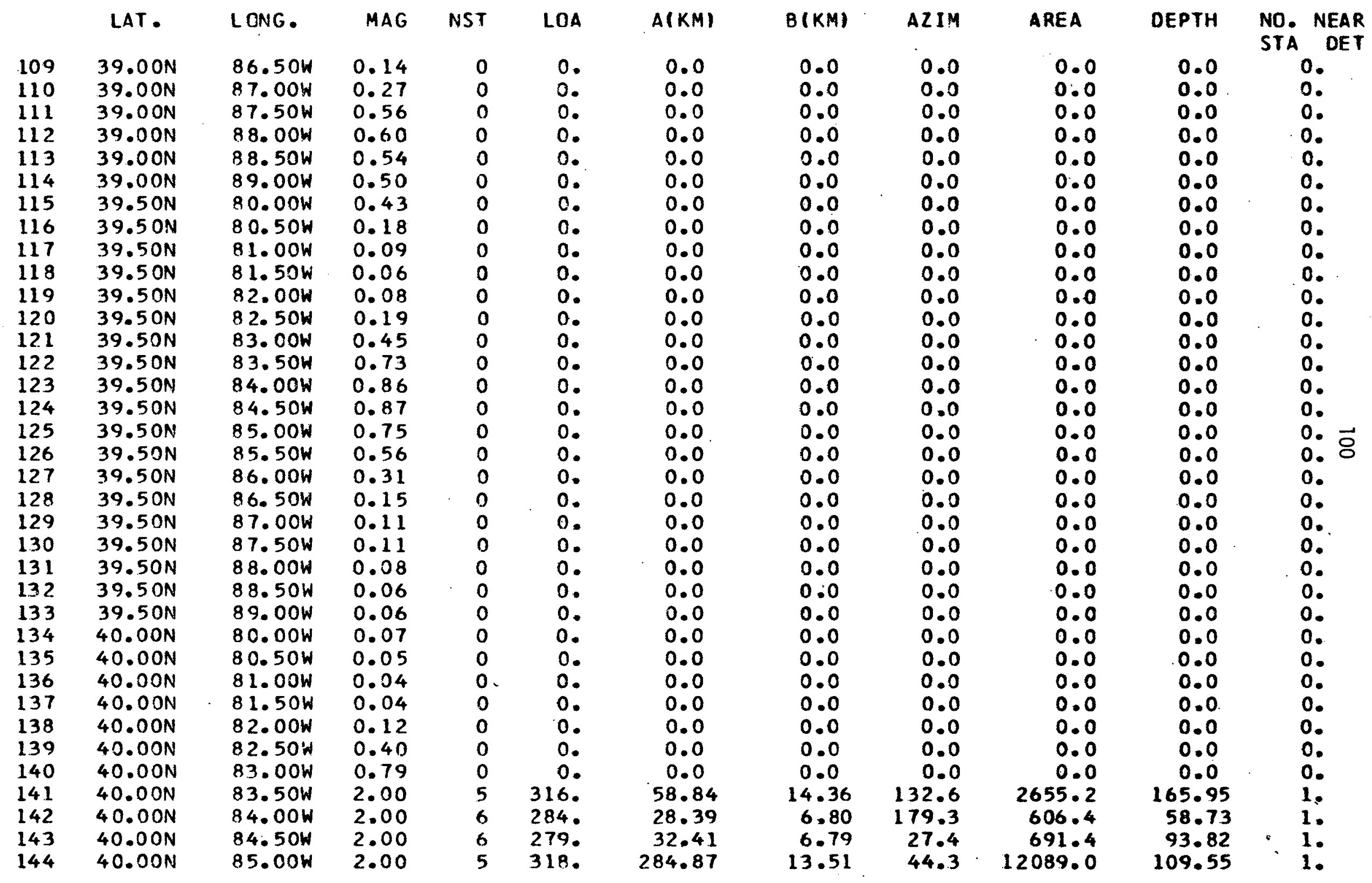




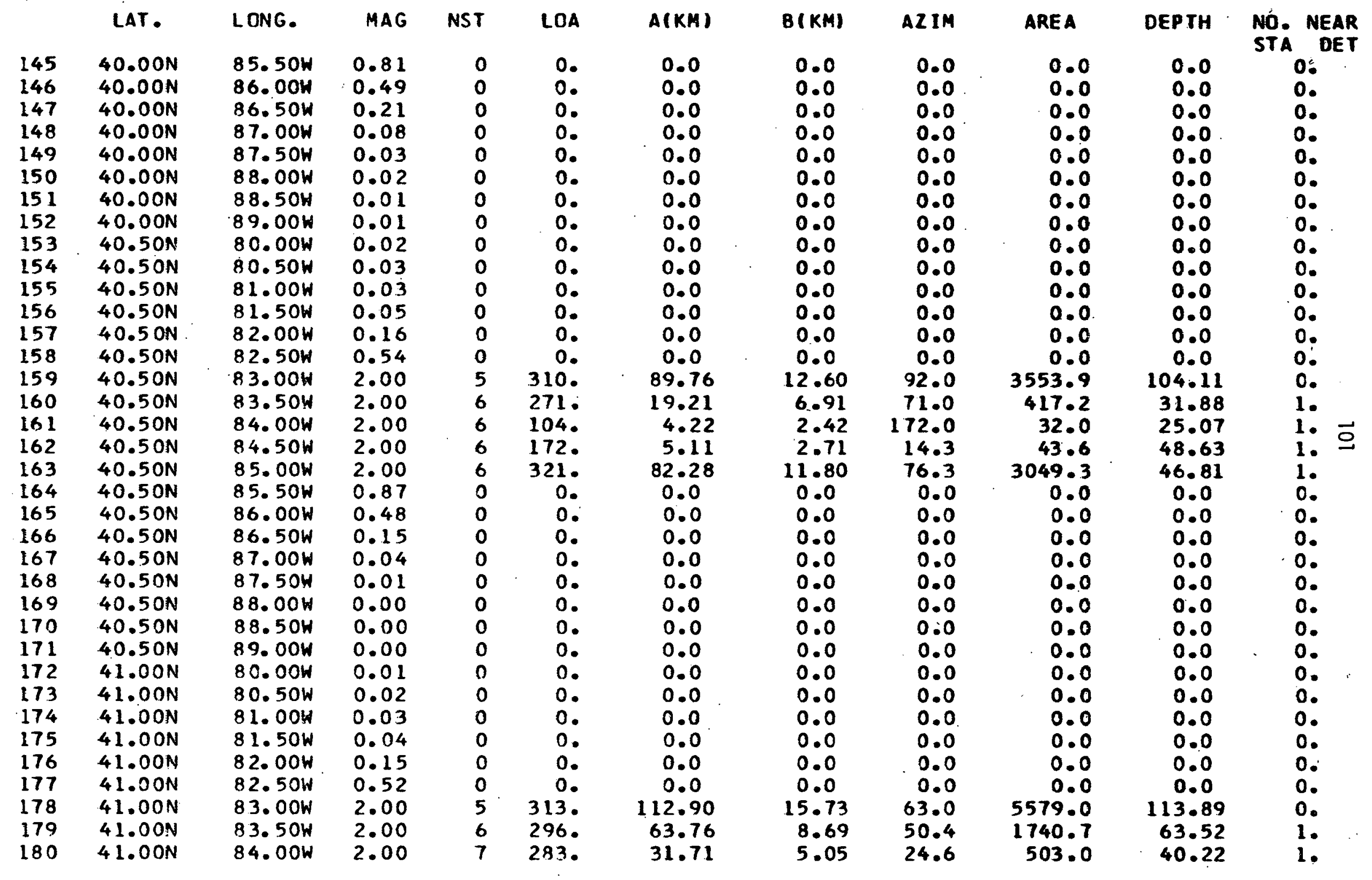




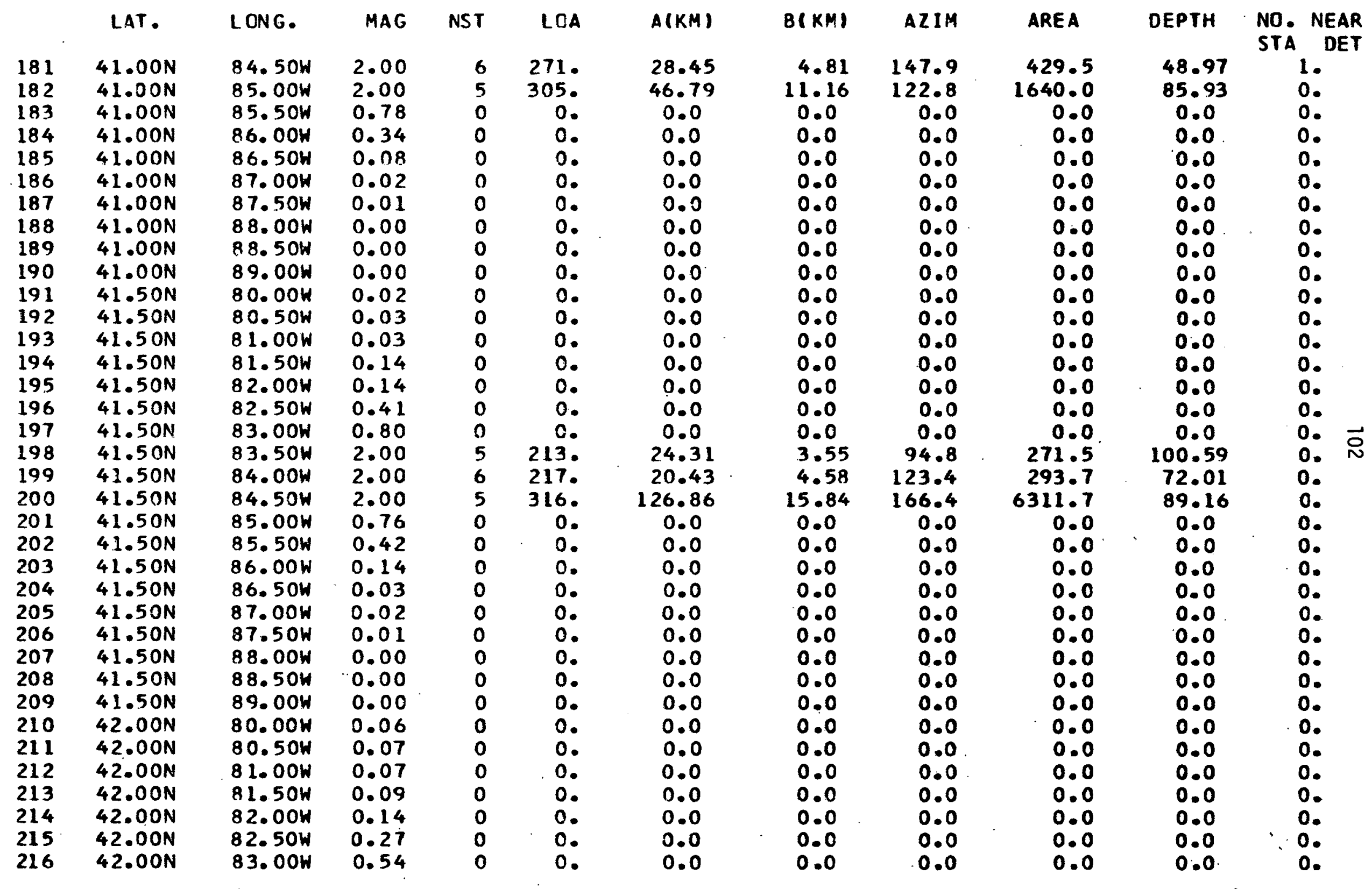




\begin{tabular}{|c|c|c|c|c|c|c|c|c|c|c|c|}
\hline & LAT. & LONG. & MAG & NST & LOA & $A(K M)$ & $B(K M)$ & $A Z I M$ & AREA & DEPTH & $\begin{array}{cc}\text { NO. NEAR } \\
\text { STA DET }\end{array}$ \\
\hline $\begin{array}{l}217 \\
218 \\
219 \\
220 \\
221 \\
222 \\
223 \\
224 \\
225 \\
226 \\
227 \\
228\end{array}$ & $\begin{array}{l}42.00 \mathrm{~N} \\
42.00 \mathrm{~N} \\
42.00 \mathrm{~N} \\
42.00 \mathrm{~N} \\
42.00 \mathrm{~N} \\
42.00 \mathrm{~N} \\
42.00 \mathrm{~N} \\
42.00 \mathrm{~N} \\
42.00 \mathrm{~N} \\
42.00 \mathrm{~N} \\
42.00 \mathrm{~N} \\
42.00 \mathrm{~N}\end{array}$ & $\begin{array}{l}83.50 \mathrm{~W} \\
84.0 \mathrm{~W} \\
84.50 \mathrm{~W} \\
85.00 \mathrm{~W} \\
85.50 \mathrm{~W} \\
86.0 \mathrm{WW} \\
86.5 \mathrm{OW} \\
87.0 \mathrm{WW} \\
87.5 \mathrm{CW} \\
88.0 \mathrm{~W} \\
88.50 \mathrm{~W} \\
89.00 \mathrm{~W}\end{array}$ & $\begin{array}{l}0.77 \\
0.75 \\
0.53 \\
0.30 \\
0.16 \\
0.06 \\
0.03 \\
0.01 \\
0.00 \\
0.00 \\
0.00 \\
0.00\end{array}$ & $\begin{array}{l}0 \\
0 \\
0 \\
0 \\
0 \\
0 \\
0 \\
0 \\
0 \\
0 \\
0 \\
0\end{array}$ & $\begin{array}{l}0 . \\
0 . \\
0 . \\
0 . \\
0 . \\
0 . \\
0 . \\
0 . \\
0 . \\
0 . \\
0 . \\
0 .\end{array}$ & $\begin{array}{l}0.0 \\
0.0 \\
0.0 \\
0.0 \\
0.0 \\
0.0 \\
0.0 \\
0.0 \\
0.0 \\
0.0 \\
0.0 \\
0.0\end{array}$ & $\begin{array}{l}0.0 \\
0.0 \\
0.0 \\
0.0 \\
0.0 \\
0.0 \\
0.0 \\
0.0 \\
0.0 \\
0.0 \\
0.0 \\
0.0\end{array}$ & $\begin{array}{l}0.0 \\
0.0 \\
0.0 \\
0.0 \\
0.0 \\
0.0 \\
0.0 \\
0.0 \\
0.0 \\
0.0 \\
0.0 \\
0.0\end{array}$ & $\begin{array}{l}0.0 \\
0.0 \\
0.0 \\
0.0 \\
0.0 \\
0.0 \\
0.0 \\
0.0 \\
0.0 \\
0.0 \\
0.0 \\
0.0\end{array}$ & $\begin{array}{l}0.0 \\
0.0 \\
0.0 \\
0.0 \\
0.0 \\
0.0 \\
0.0 \\
0.0 \\
0.0 \\
0.0 \\
0.0 \\
0.0\end{array}$ & $\begin{array}{l}0 . \\
0 . \\
0 . \\
0 . \\
0 . \\
0 . \\
0 . \\
0 . \\
0 . \\
0 . \\
0 . \\
0 .\end{array}$ \\
\hline
\end{tabular}


VEAR STATIOA DETBCFION

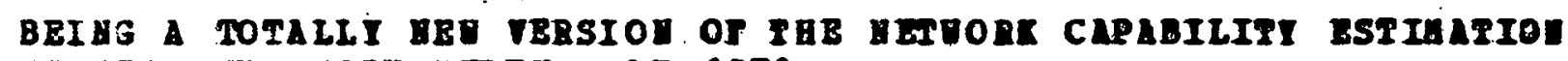
PBOGRAM E BART IITE, AAI 1970

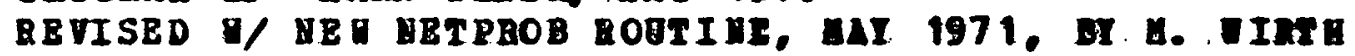

MOT B: COMUENT CARD C'S HAVE BEE ADDBD TO CALI SOBROUTI UBS TAAT DO HOT BXIST OH OUR SISTEH RATHER THAY REAOVIMG THE STATEAET TO DAIUTAIY THE IYTBGRITY OF THE ORIGIHAI PROGRAB. $2 / 21 / 80$

EXA UPLE OP. RUE CALI TO BET YOBTH

\$BU YBT GORTE.O 4=IAPUT (STATFOA DATA FILES) 5=IBPOT (DATA AND TABLBS) 6=OUTPOT (POR PRIATEB) 7=OUTPOT (POR PLOT PILE)

THIS LISTING OP THE PROGRAE YETWORTH IS SET UP POR THE USE JP THE UNI VERSITY OF MT CEIGAE SEISHIC OBSERV ATOBY AHD IS MOT AEANT TO BE DIR BCTLY TEAISFERABLE TO OTHER SYSTBES AT THIS TI HB. SOME PARTS OP THE ORIGIMAL PBCGBAH BY H. IIRTH 1971 HAVE BEB: TOT ALLY REHOY BD.

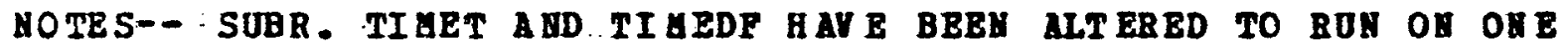
PART ICULAR SET OF. UYEVEMLY SPACED TRAVEL TIEE DATA. IF UEU DATA IS TO BE USED THESE SUBR. SHOULD BE ALTERED.

-THE SP GAGNTTUDE CORB. MRITE STATEUEATS A AVE BEEU ALTEBED FOR THE TECHEICAL REPOBT AED GOST BE CHANGED IF IUPOT DATA IS CEANGE D. 


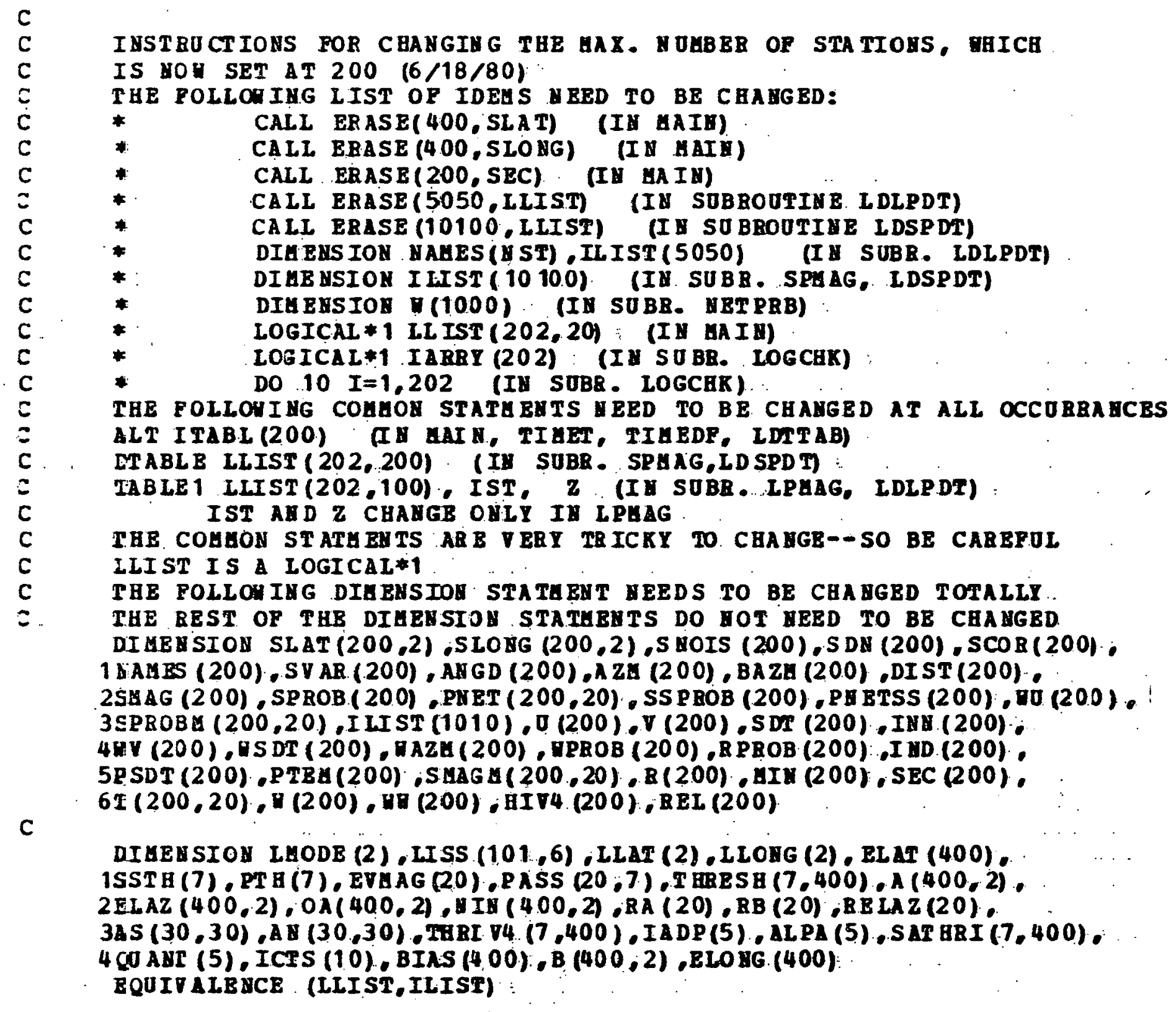


BEAL*8 LEODE, HALES, LTAB, ALPA, IDA, IDB, IDC

BEAL*8 COHB1, COHB2, COHB3

COULON/ALT/. ITABI (200) .. IALT

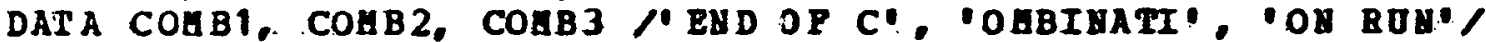

DATA ALPA /'DEPTH PK', 'DEPTE HP!, 'AXIS HPK'.'AXIS IP'.

1 - MEAR STA'

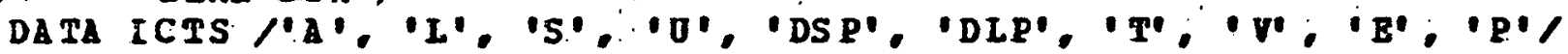

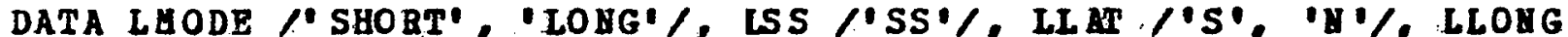

1 I B., In,

LOGICAL*1 LLIST $(202,20)$, LLF

$\operatorname{LLF}(X)=X \cdot G E .0$.

$\operatorname{ABEAP}(X, Y)=3.141593 * X * Y$

IOOT $=999$

$I X=777773777$

CALL BBASE (4.00, SLAT)

CALL EBAS E (400, SLONG)

C

$10 \mathrm{BBAD}(5,1890, \mathrm{EXD}=1870)$ I DA, IDB, IDC, (QU AUT (I), I=1,5)

DO $20 I=1,5$ IP (QUART (I) . EQ . 0.) QUABT (E) = $1.0 \mathrm{E} 67$ 20 COXTIUUB

$$
\text { A - CARD }
$$

READ (5, 1920) LAB, LAPHA, SDSIG, HODE, NOHAG, BHAG, DHAG, PTH, IIADP . ICOEB, IHBIAS

IF (LAB . BB. ICTS (1)) :G0 TO 450

$\operatorname{IDAP}=\operatorname{IADP}(1)+\operatorname{IADP}(2)+\operatorname{IADP}(3)+\operatorname{IADP}(4)+\operatorname{IADP}(5)$

BS D $=0$

NRE $=0$

NRAUDS $=0$

c

40 VRITE $(6,1970)$. LAPHA, LHODE (MODE), S DSIG, PTH

L - CARD

BEAD (5, 19.80) LAB, ELL, EL VAR, PELI, IF PREL 


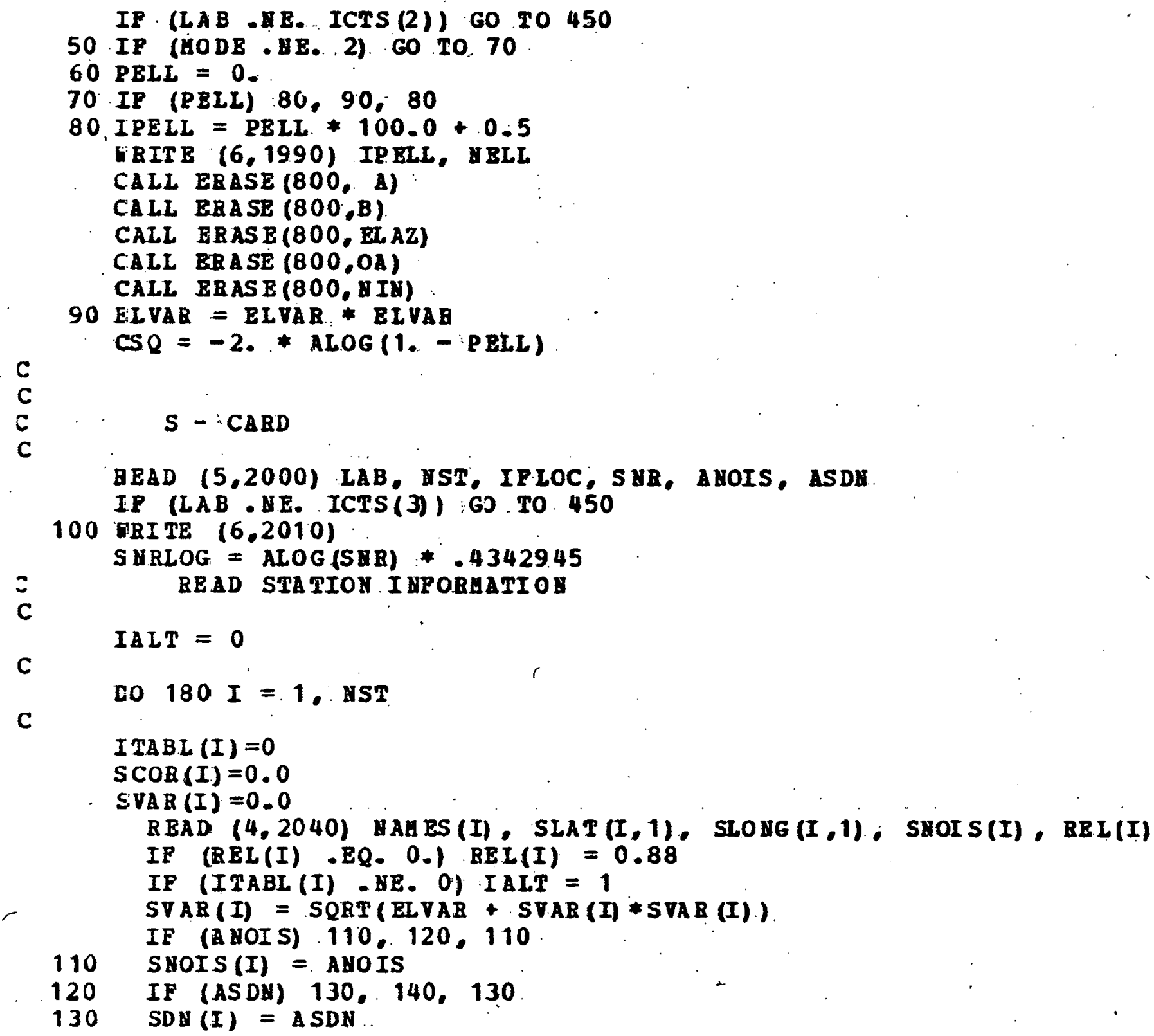




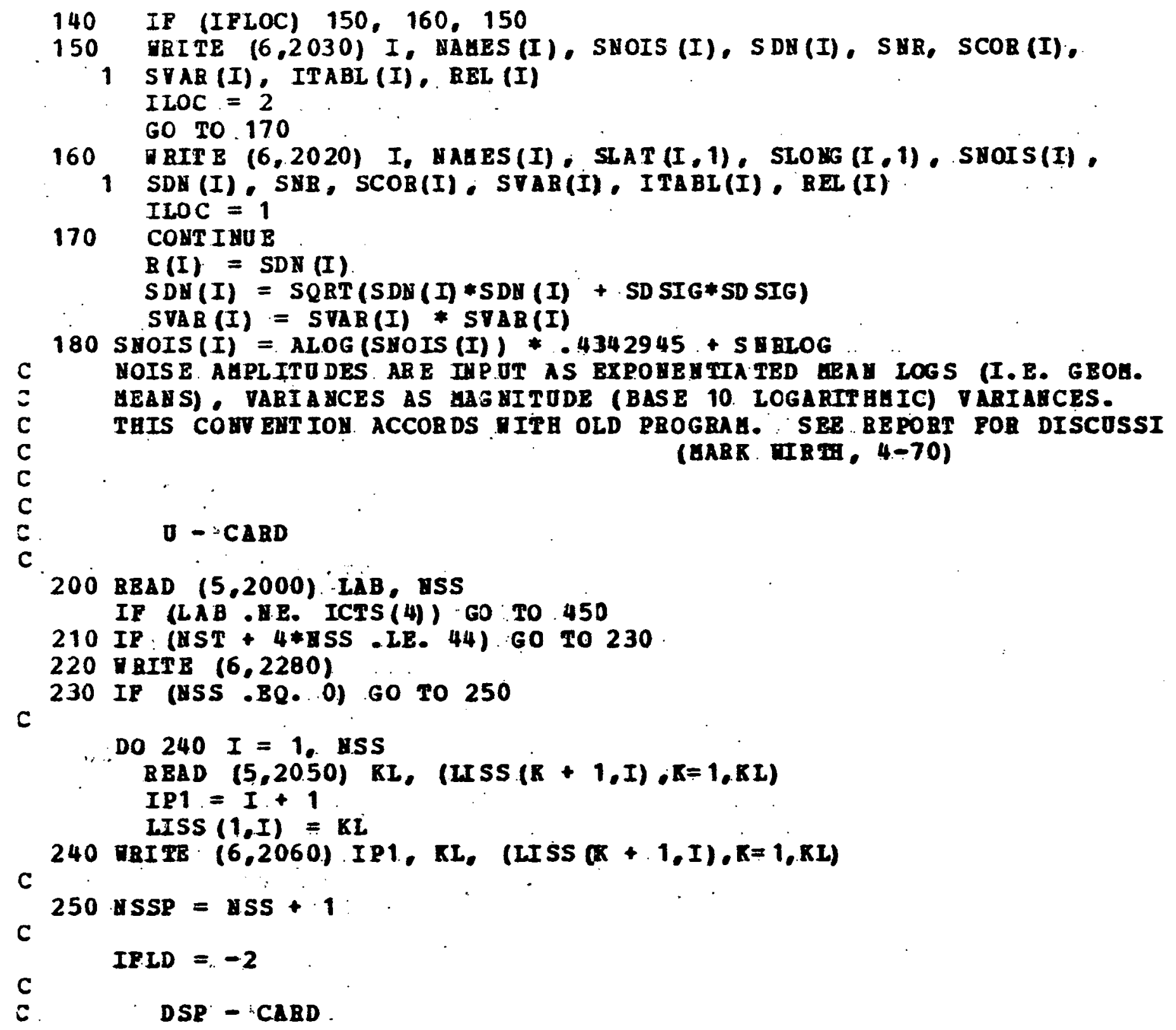




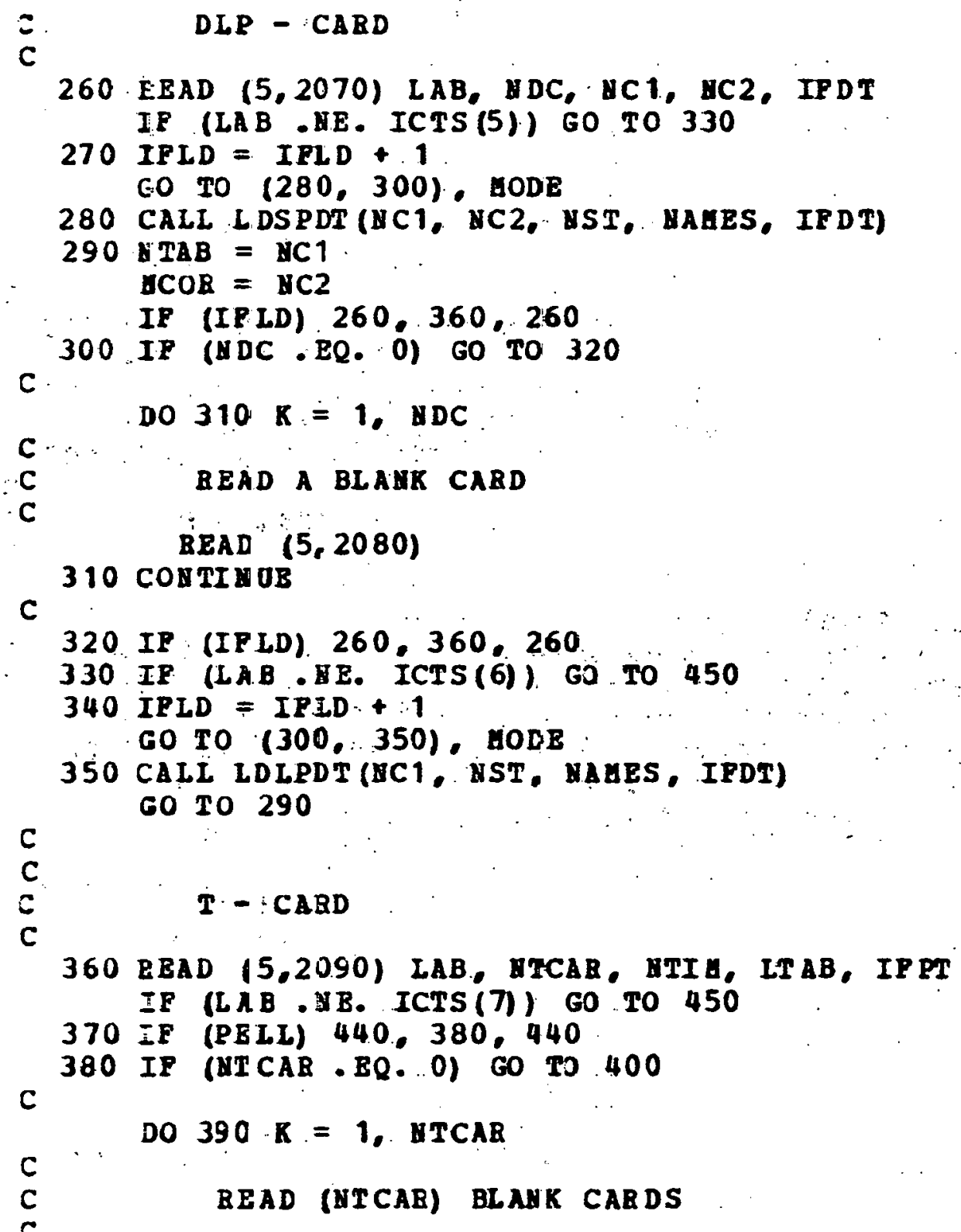




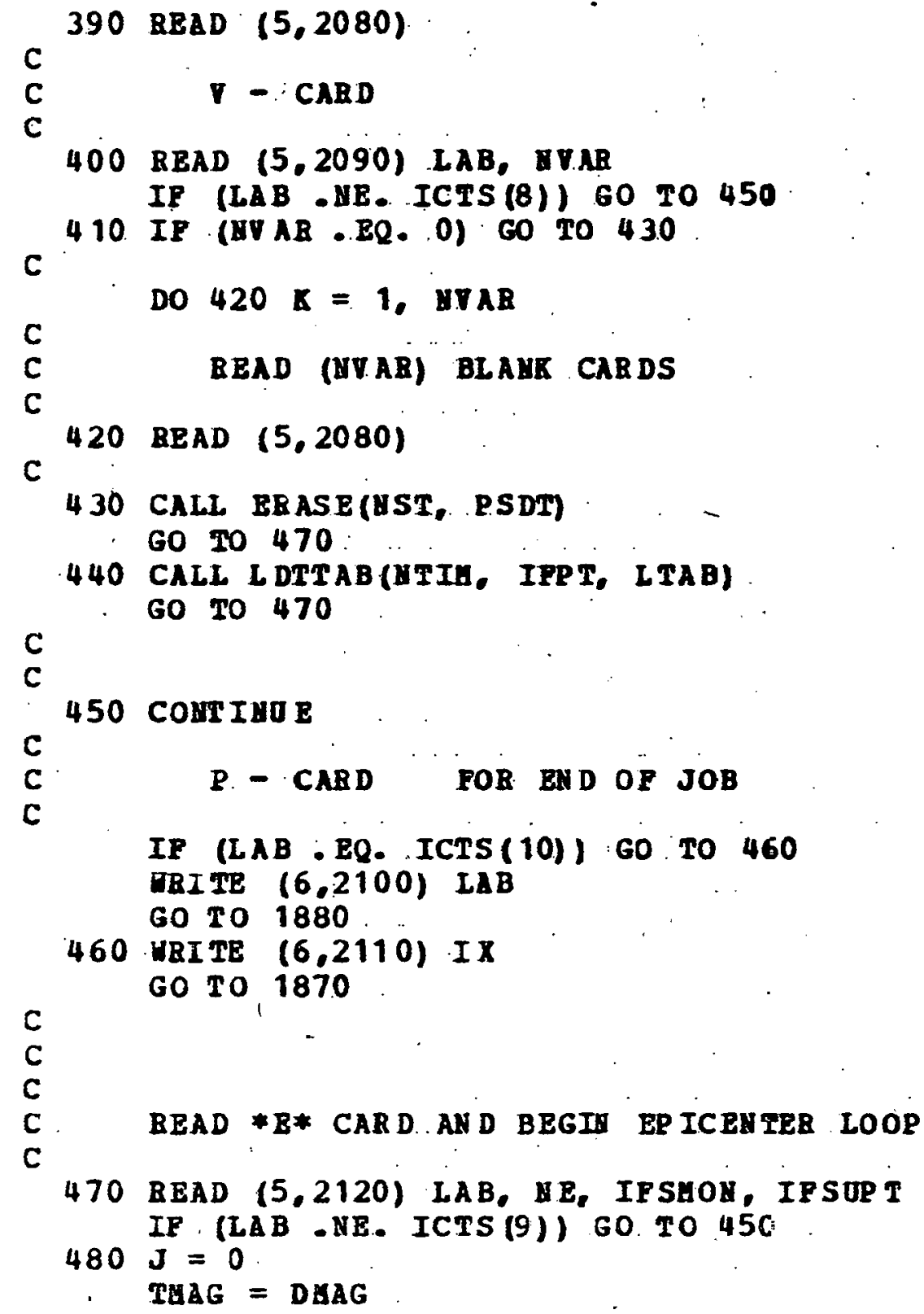


C

CALL ERASB (400, BIAS)

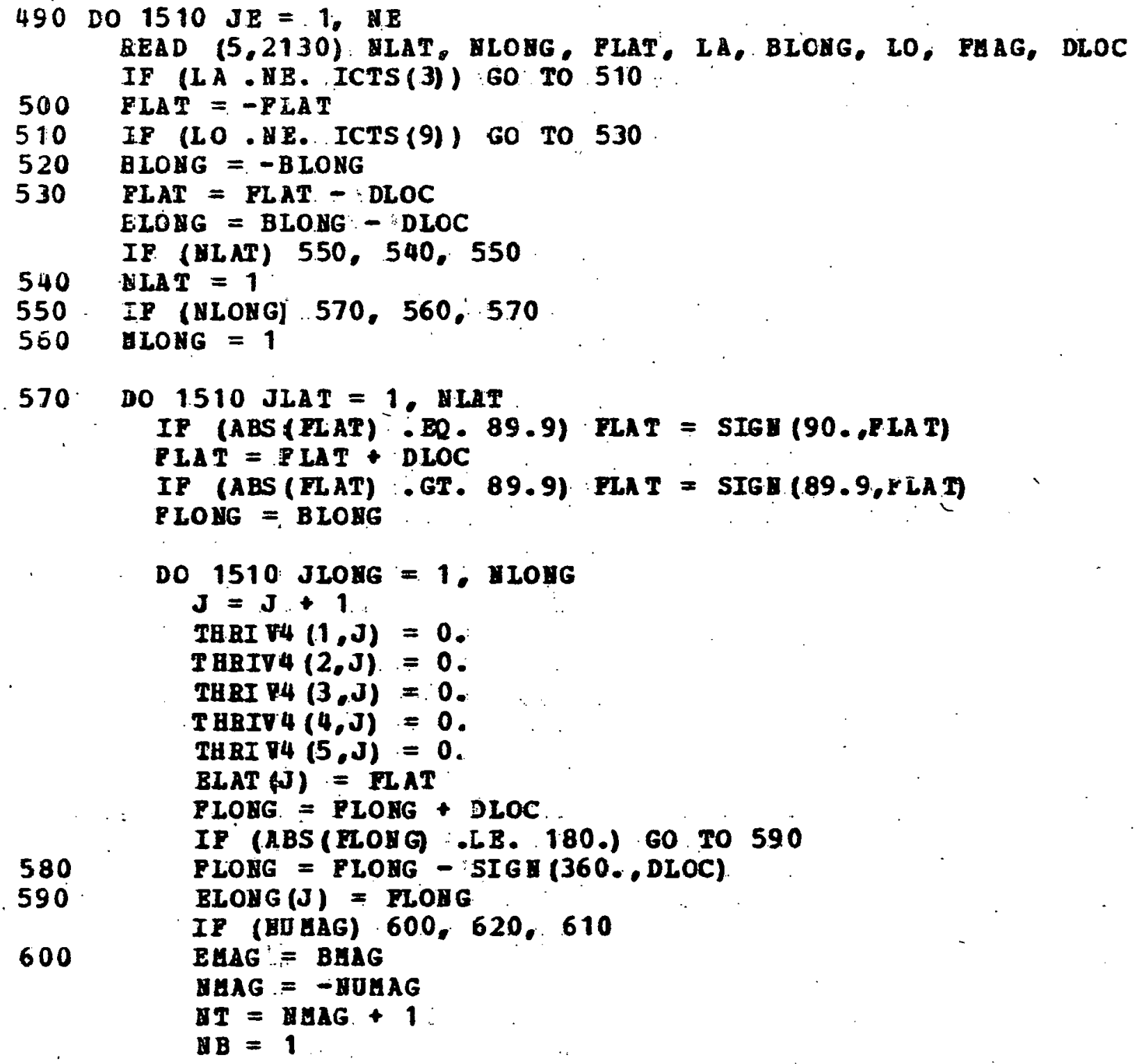

C 


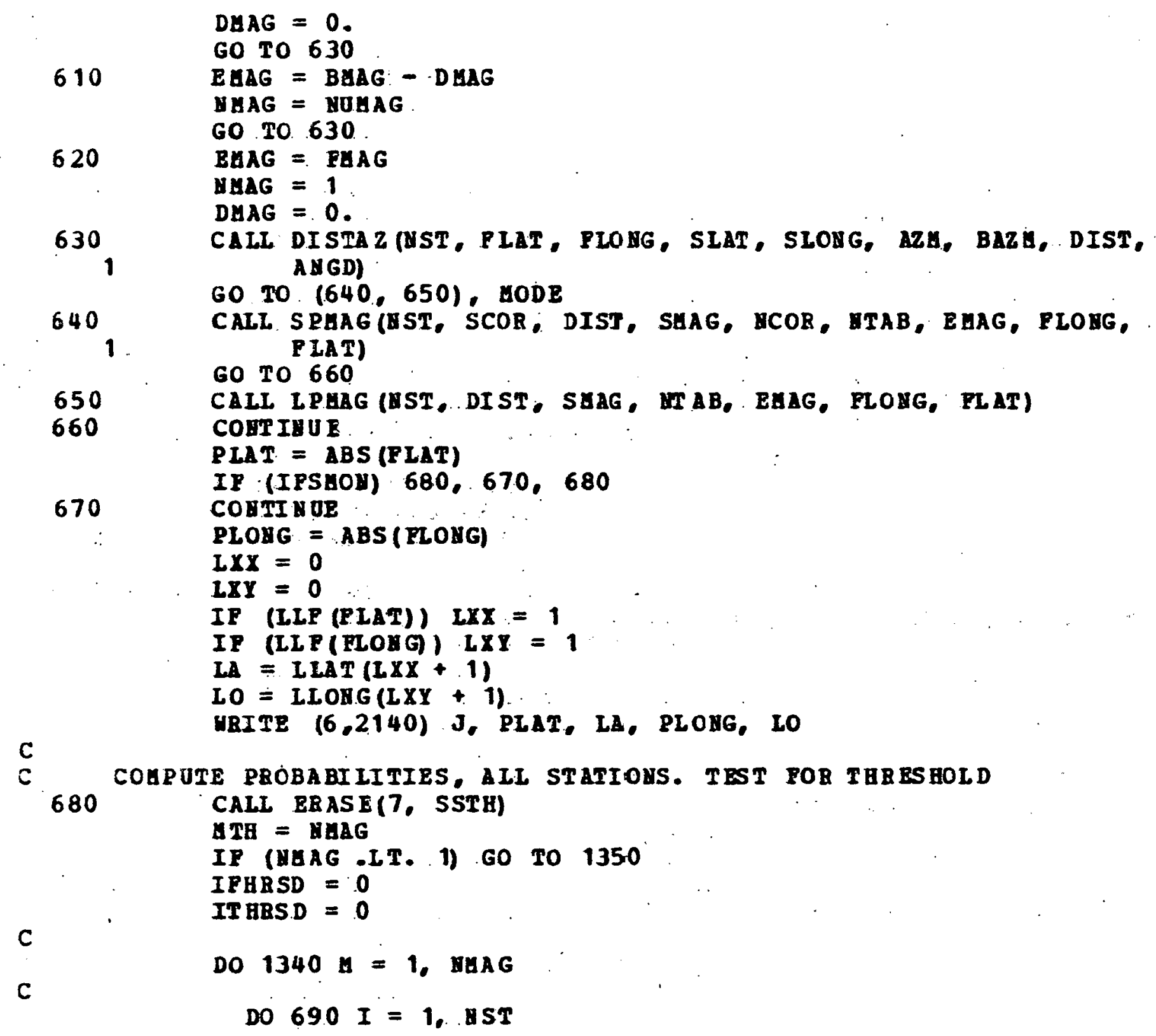




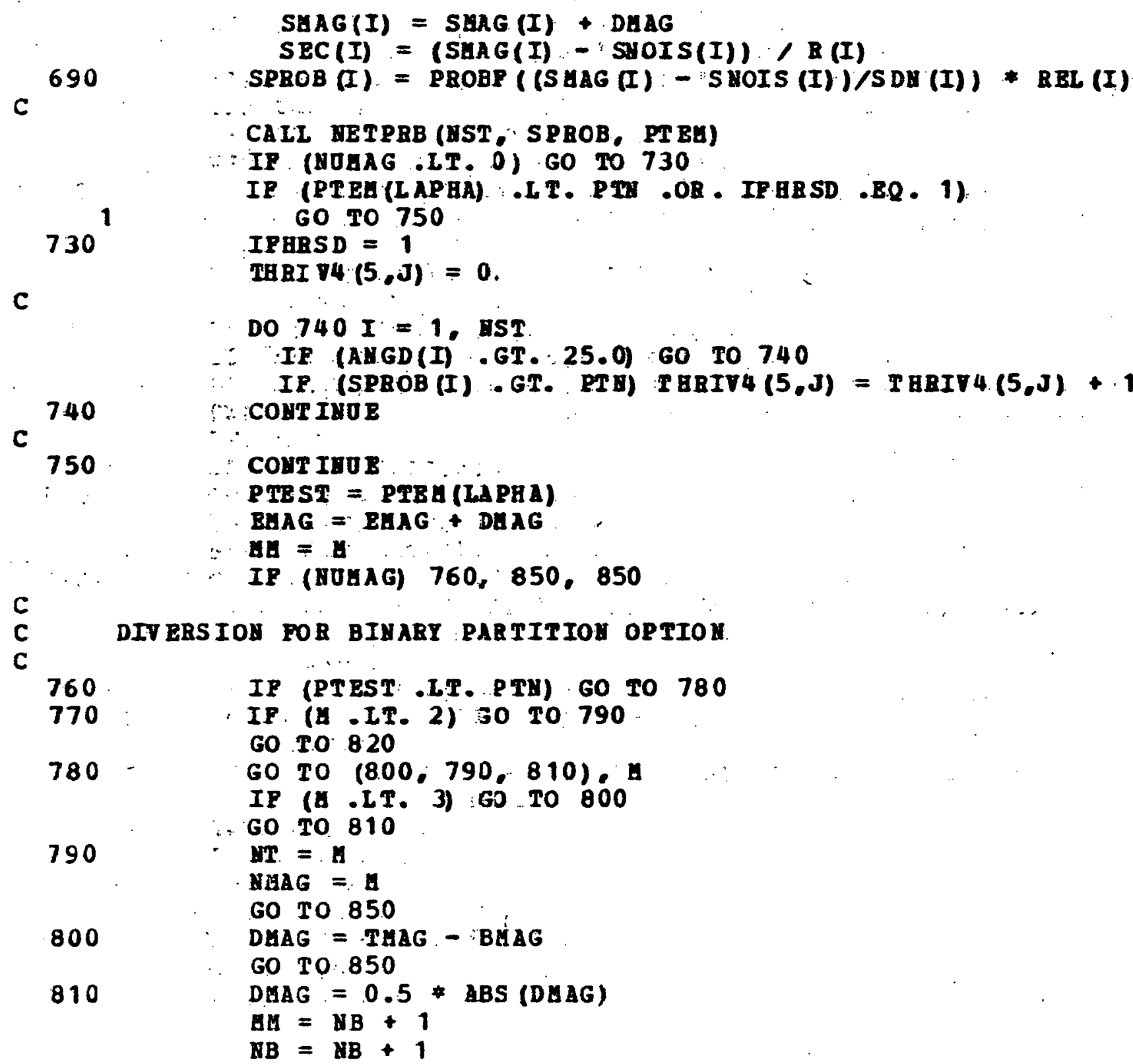




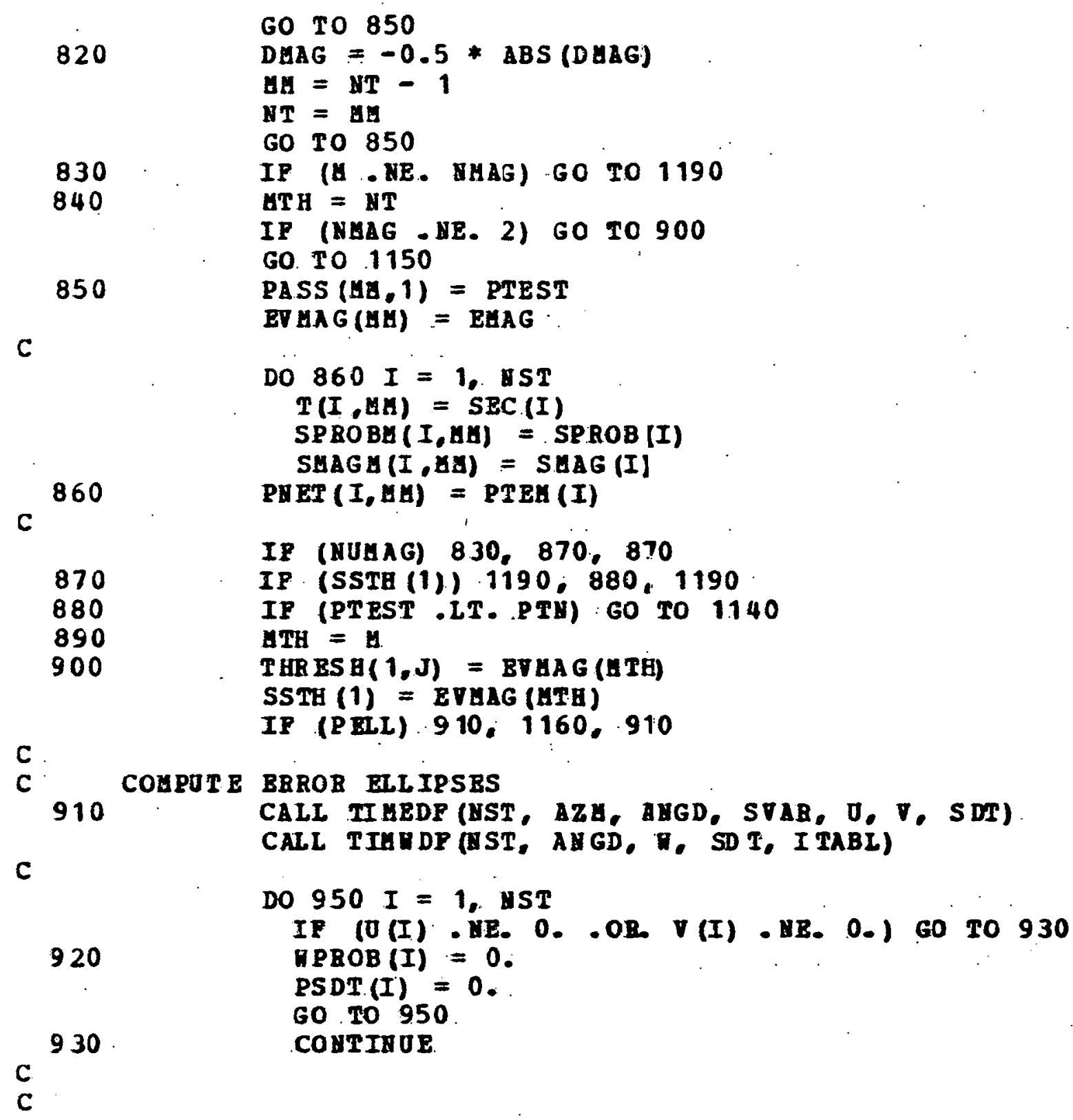




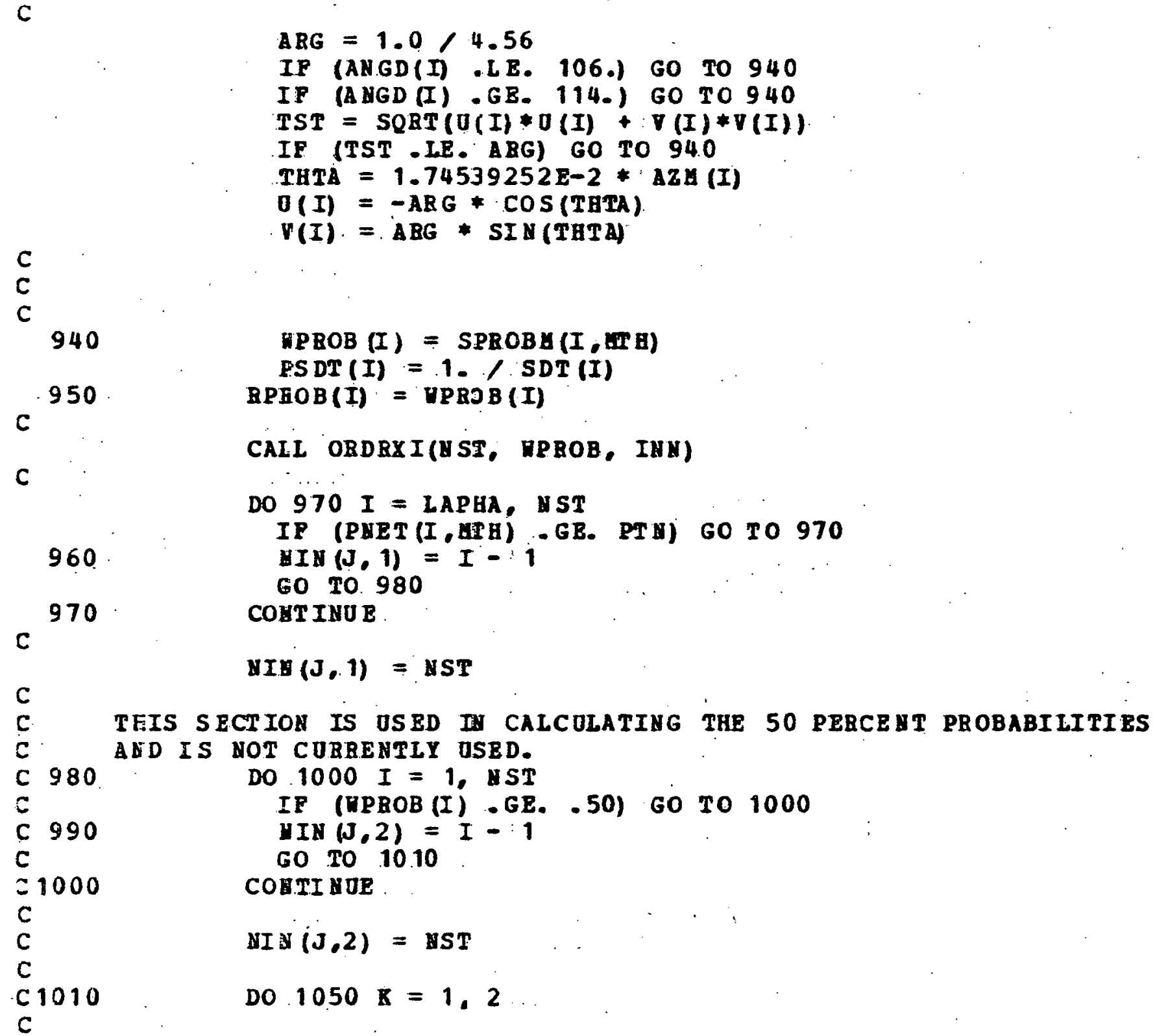




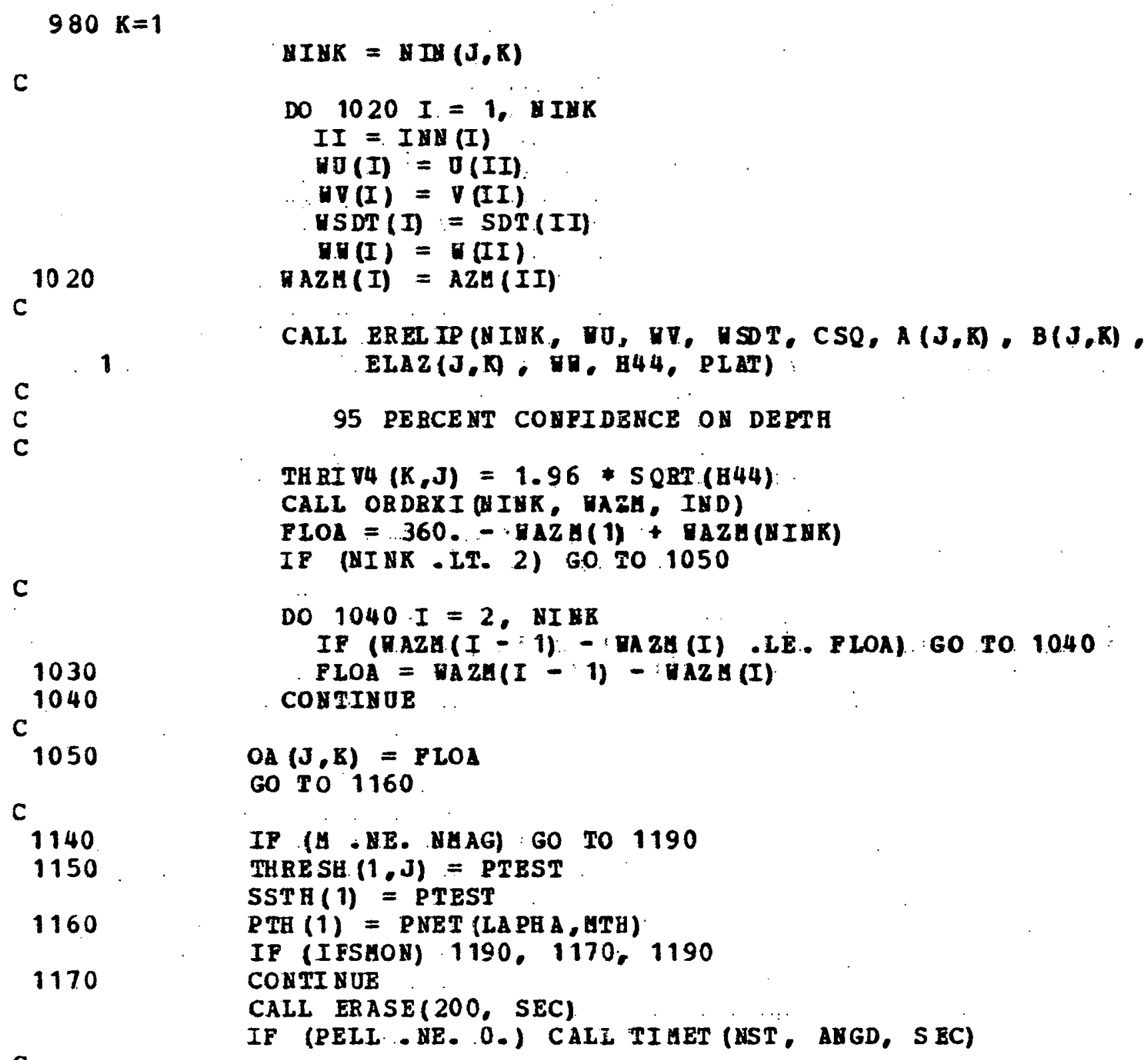




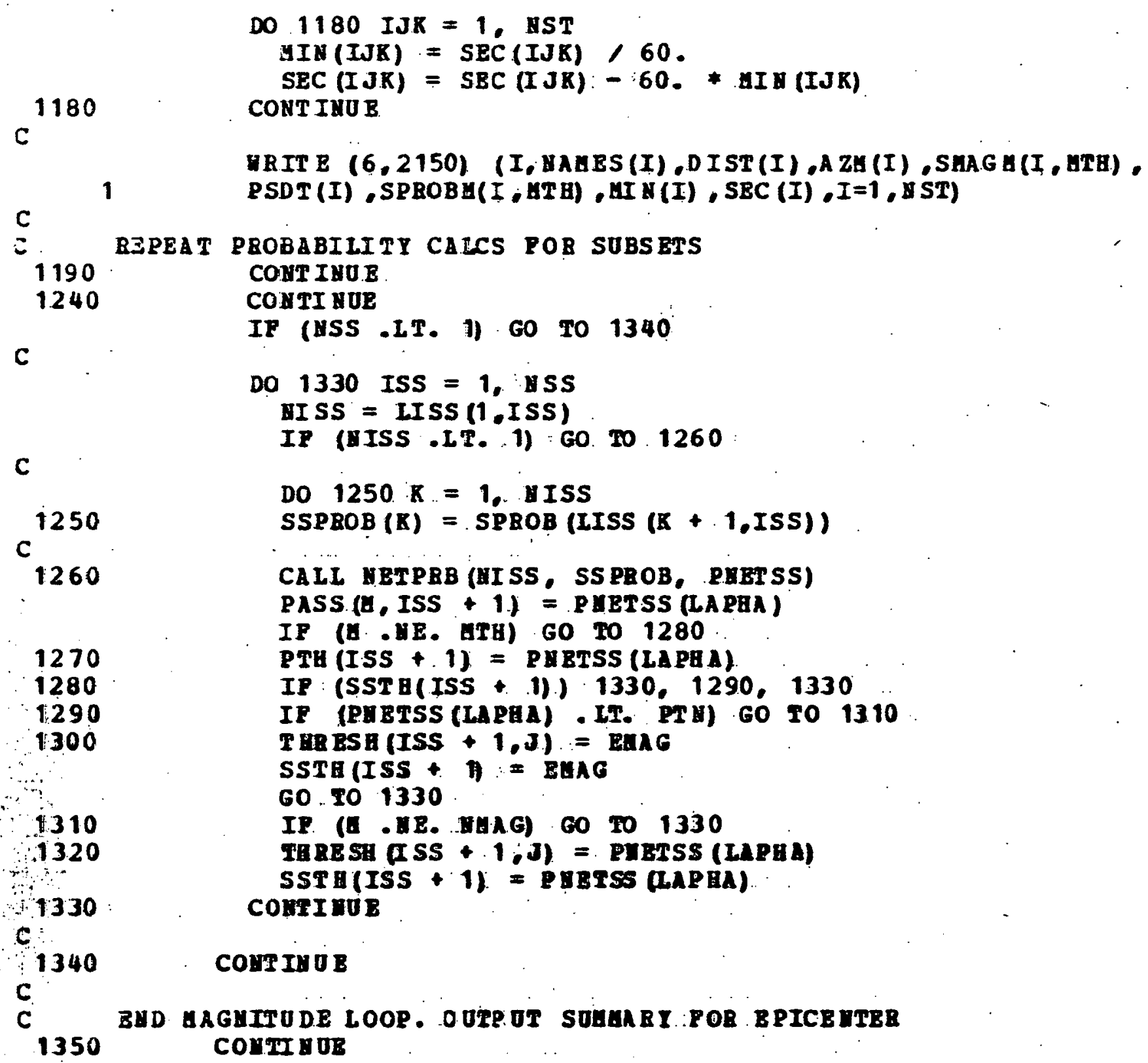




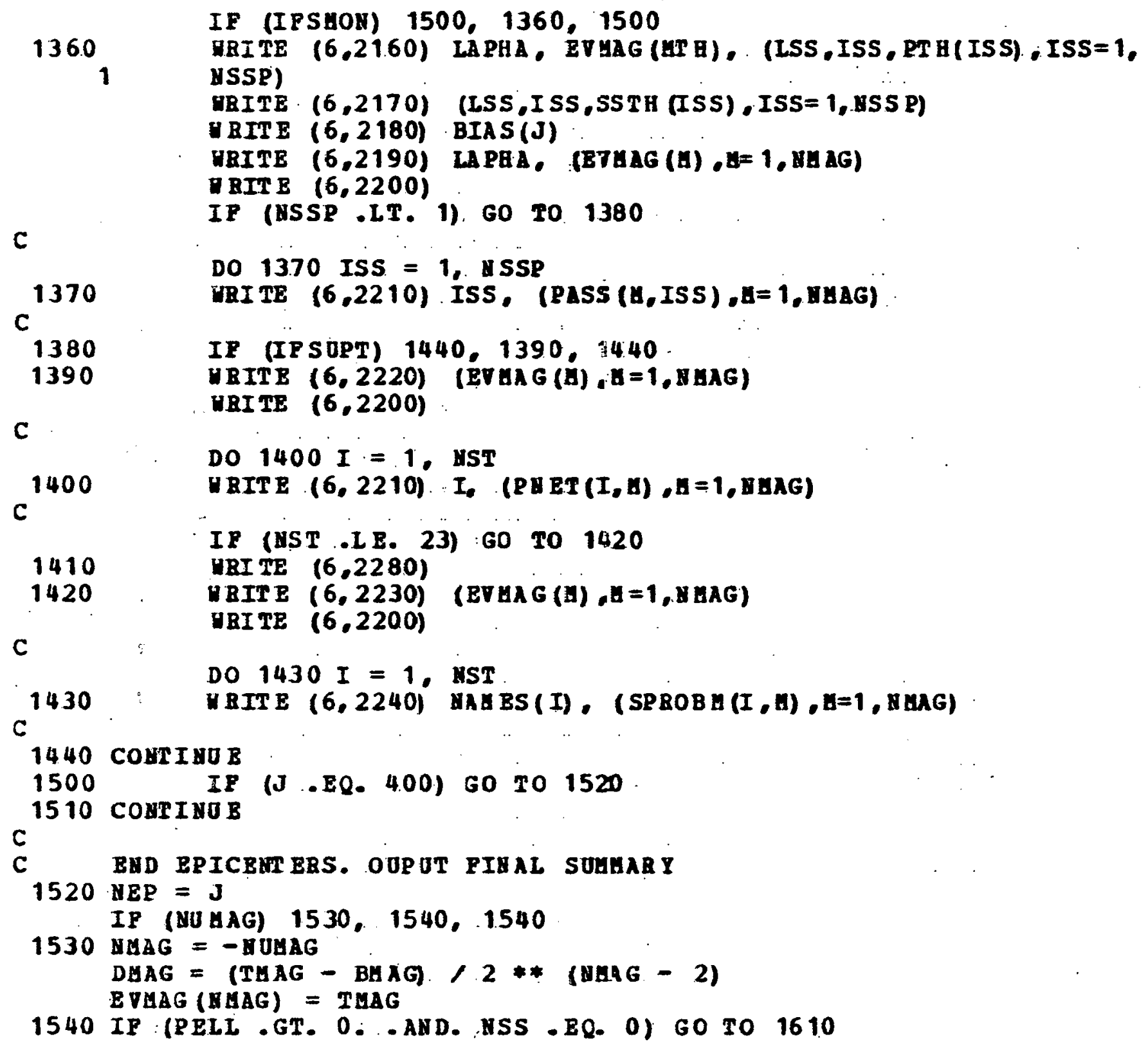




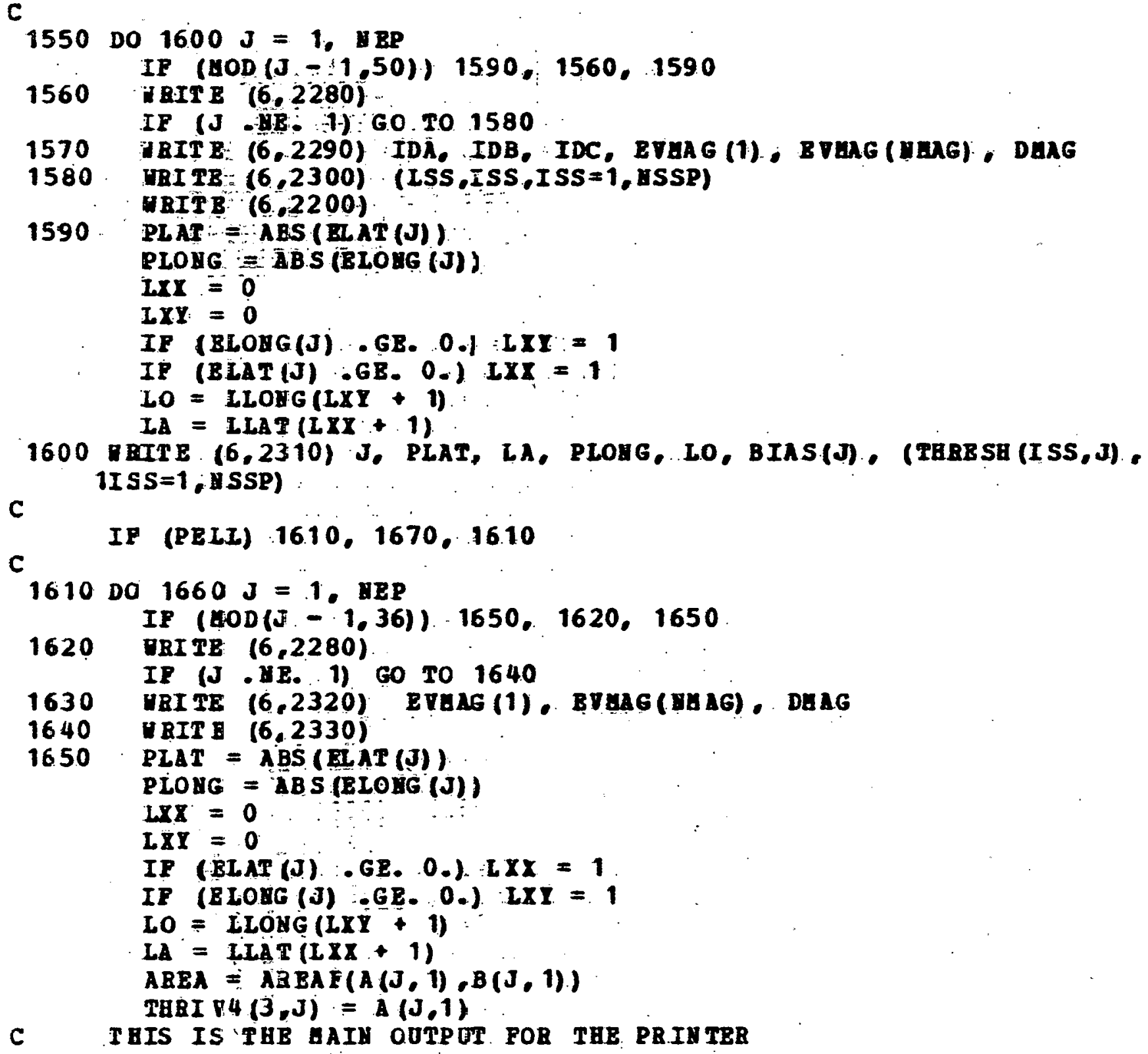




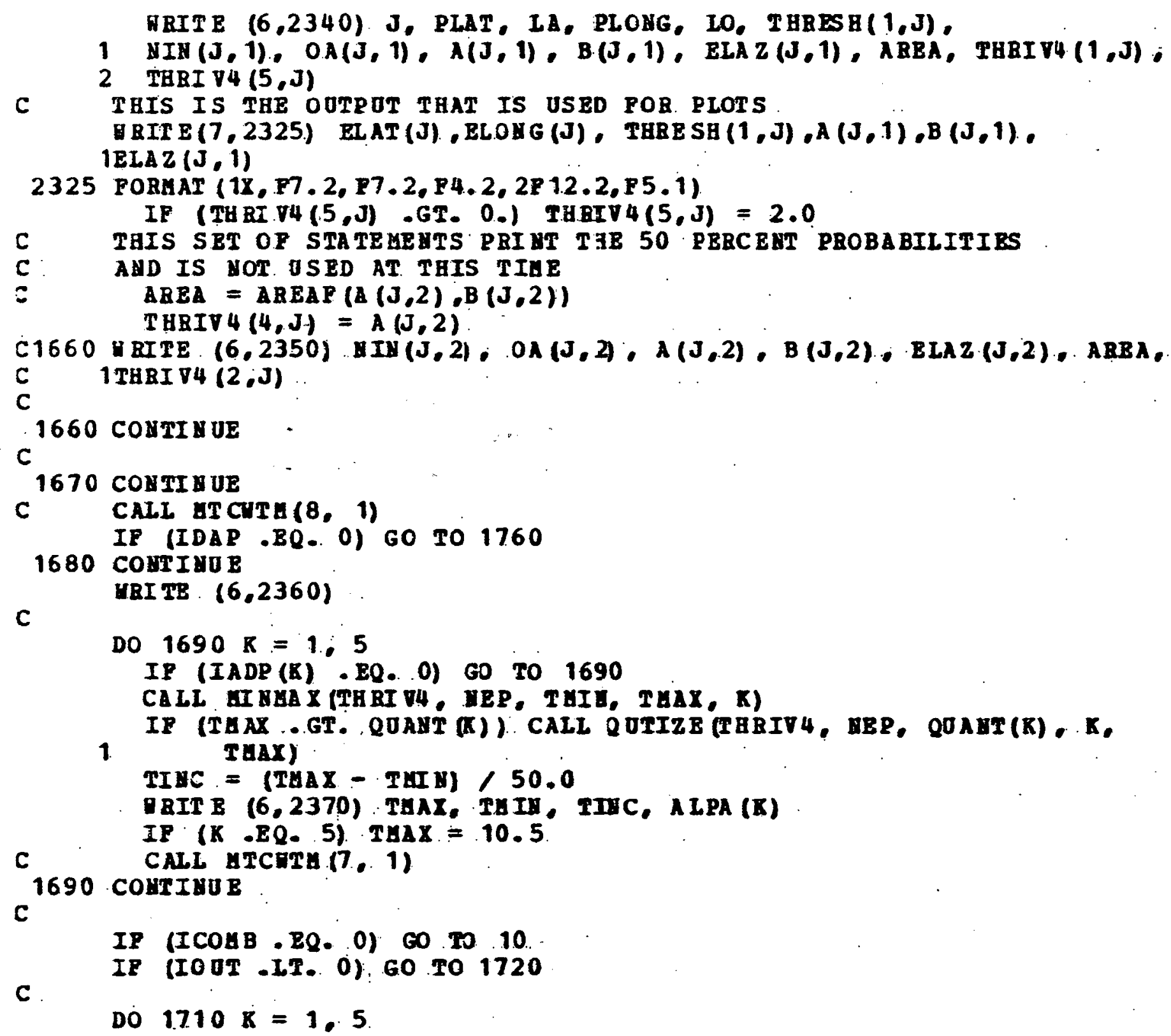


c

IF (I ADP (K) EEQ . 0) GJ TO 1710

1700

DO $1700 \mathrm{~J}=1$. $\mathrm{BEP}$

$\operatorname{SATHRI}(\mathrm{K}, \mathrm{J})=\operatorname{THRIV4}(\mathrm{R}, \mathrm{J})$

c

1710 continue

IOUT $=-999$

GC. TO 10

1720 CONIIHUE

DO $17.50 \mathrm{~K}=1,5$

C

IF (IADP (K) EQQ. O) GO TO 1750

DO $1730 \mathrm{~J}=1, \mathrm{NEP}$

THEIV $4(K, J)=\operatorname{SATHRI}(K, J)-\operatorname{THRIV4}(K, J)$

1730 CONTI NUE

C $T$.

CALI MINHAX (THBI V4, NEP, THIN, THAX, R)

IF (THIN . GT. 1.5) GO TO 1750

WRITE $(6,2370)$ THAX, THIX

c

THIN $=\mathrm{ABS}$ (TMIN) +1.5

1740

DO $1740 \mathrm{~J}=1$, NEP $\operatorname{IHRIV} 4(K, J)=\operatorname{TRRIV} 4(K, J)+\operatorname{THIH}$

c

1750 CJNTI JE

c

$$
\begin{aligned}
& \text { ICOAB }=0 \\
& \text { IOUT }=999 \\
& \text { IDA }=\text { COMB } 1 \\
& \text { IDB }=\text { COBB2 } \\
& \text { IDC }=\text { COAB3 } \\
& \text { GO TO } 1680
\end{aligned}
$$

1760 CONTI YUE

GO TO 1780 


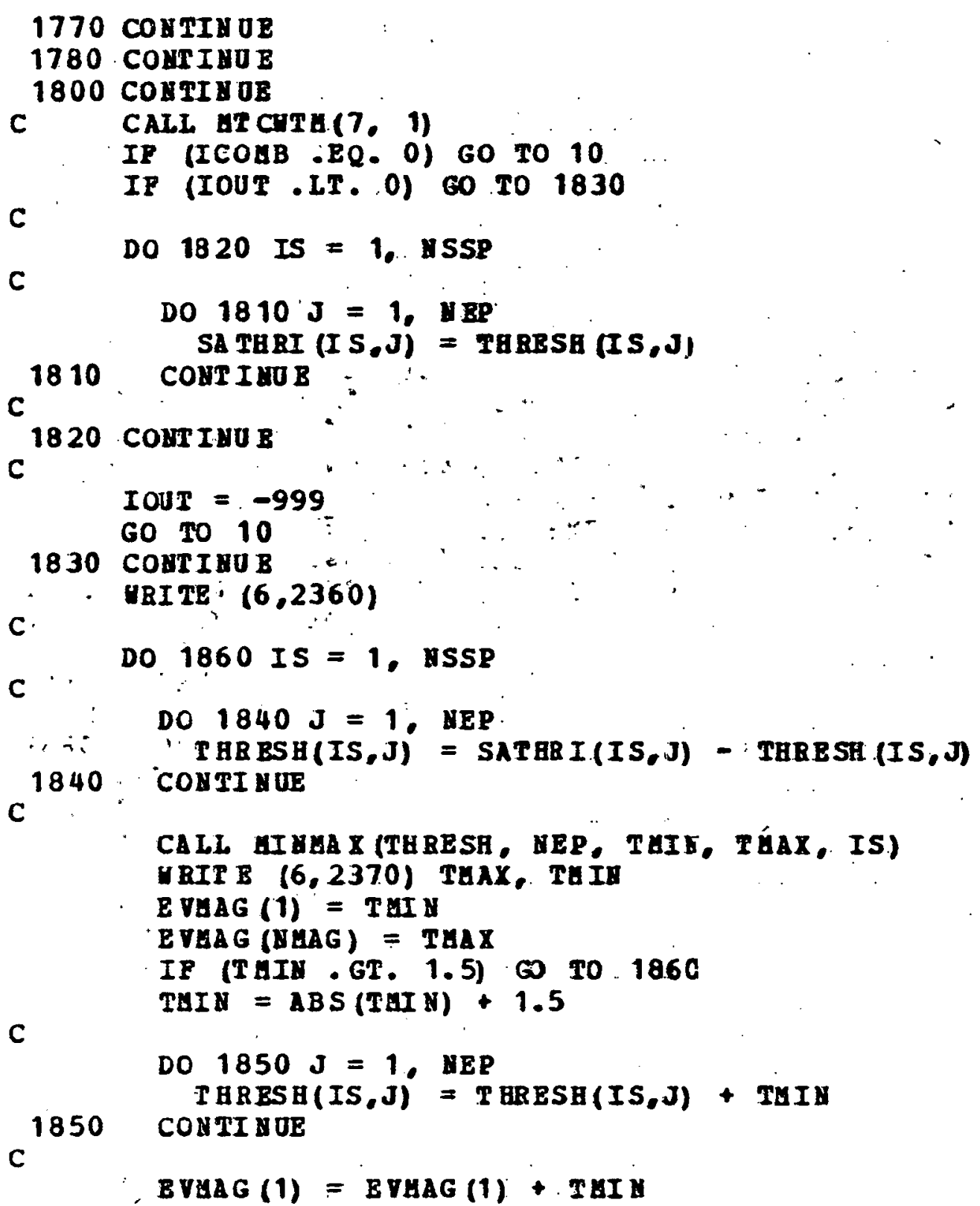




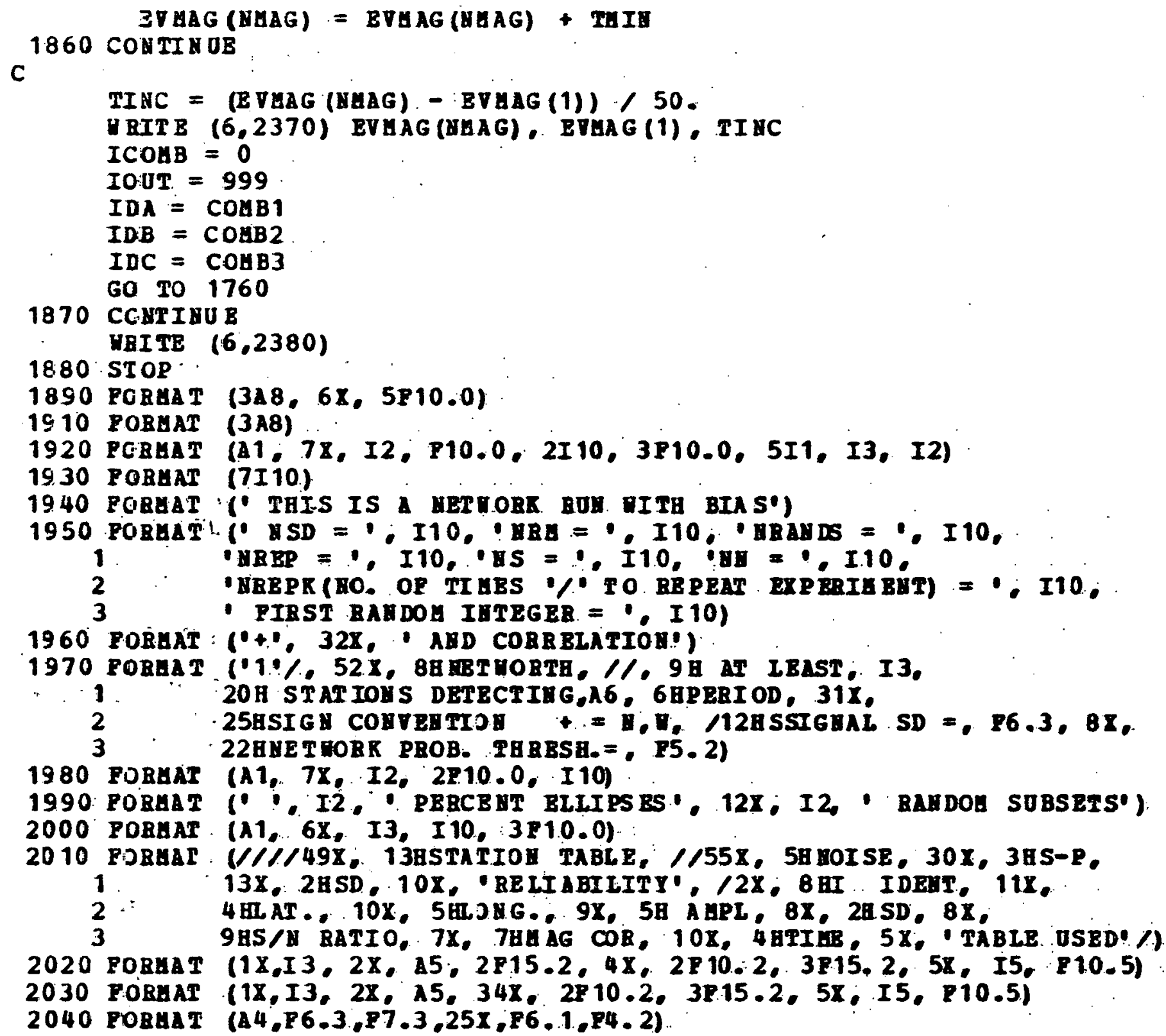




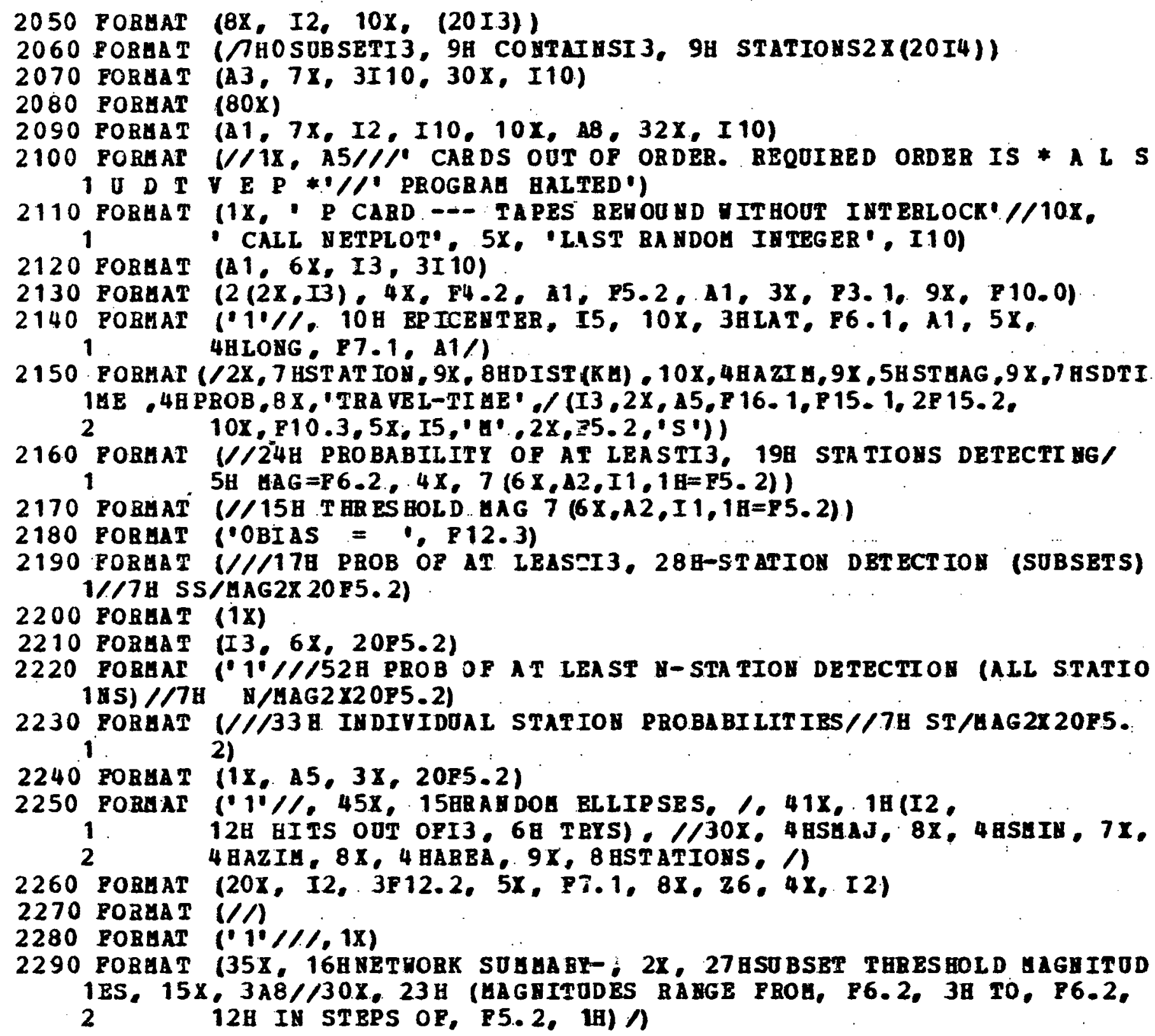




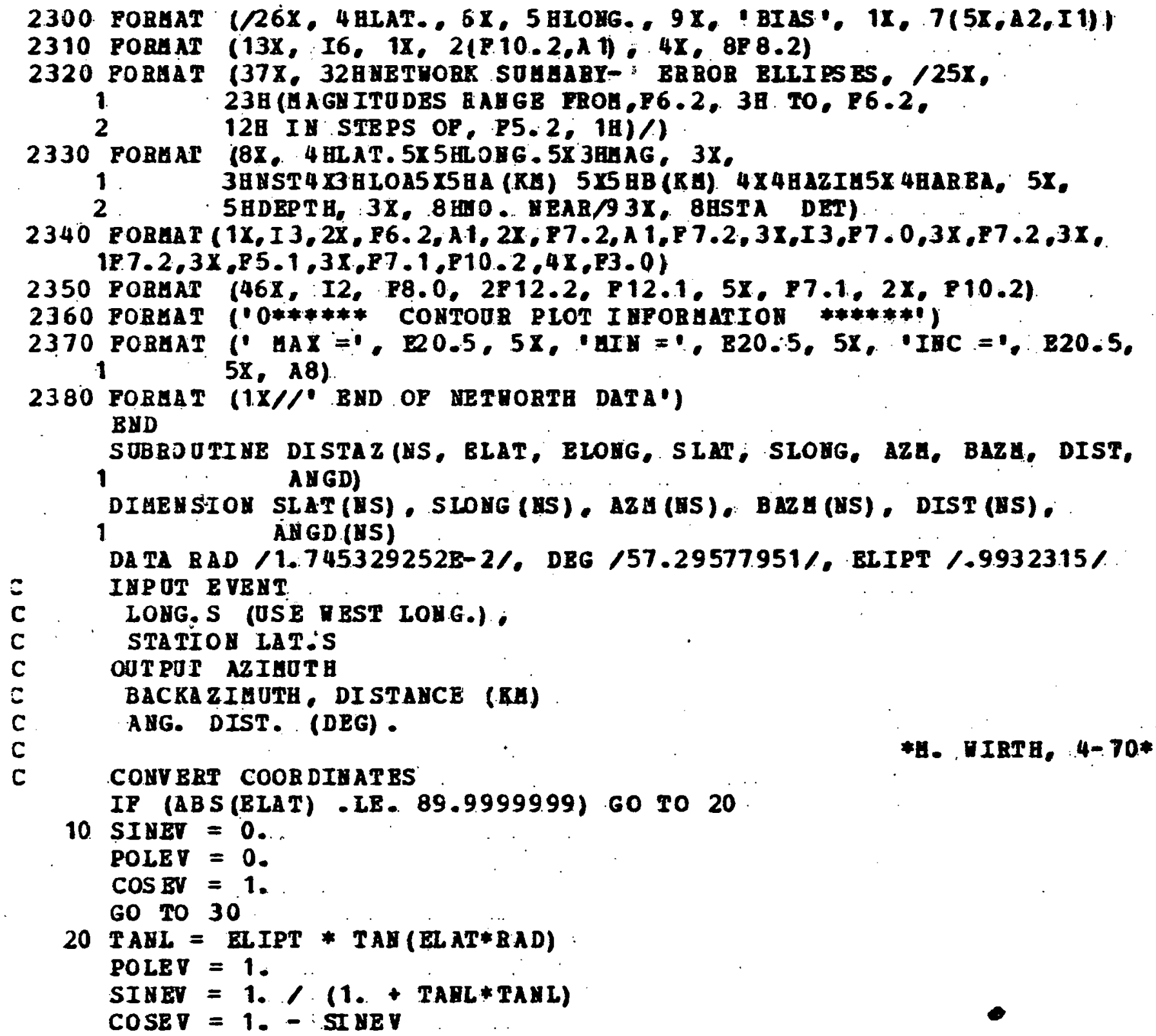

*A. UIRTB, 4- 70* 
SIUEV = SQRT (SIHEV)

COSE $=$ SQRT (COSEV)

30 IP (BL AT) $40,50,50$

C

$40 \cos V=-\cos 3 \mathrm{~V}$

50 DO $310 I=1$, HS

DLOHG = POL ET * ABS (BLOBG - :STOBG (I))

SGEL $=-P O L B V$

IP (DLOIG - 180.) 80,60,70

60 SGEL $=0$.

GO TO 80

70. DLONG $=360 .-$ DLORG

$S G H L=1$.

80 DLOHG = DLOHG $*$ BAD

IF (ABS (SLAT (I)) .LE. 89.9999999). GO TO 100

90 SIMST $=0$.

DLONG $=0$.

SGRL 0 .

$\operatorname{cossT}=1$.

GO TO 110

100 TALI = ELIRT * TAR (SLAT (I)*BAD)

SIMST $=1 . \% \cdot(1 .+$ TARL*TABL $)$

$\cos S T=1 .-$ SIBST

SIRST $=$ SQBT. (SI RST)

$\operatorname{COSST}=\mathrm{SQBT}(\mathrm{COSST})$

110. IF (SLAT(I)) $120,130,130$.

$120 \operatorname{coss} \mathrm{T}=-\operatorname{cossT}$

c

COHPUTE EVEH I-STATION POLAR TRIABGLE

130 COSANG = COSST *COSEV * SIUST *SIHEV * COS (DIOHG)

IF : (ABS.(COSANG) . IT. 1.) GO TO 160

$140 \mathrm{AZH}(I)=0$.

$\operatorname{BAZH}(I)=0$.

$\operatorname{AYGD}(I)=0$.

IF (COSANG) 150, 310, 310

$150 \quad \operatorname{ANGD}(I)=180$.

GO TO 310

160 AMGD (I) = ARCOS (COSAMG)

$S I N A=S I N(A N G D(I))$ 


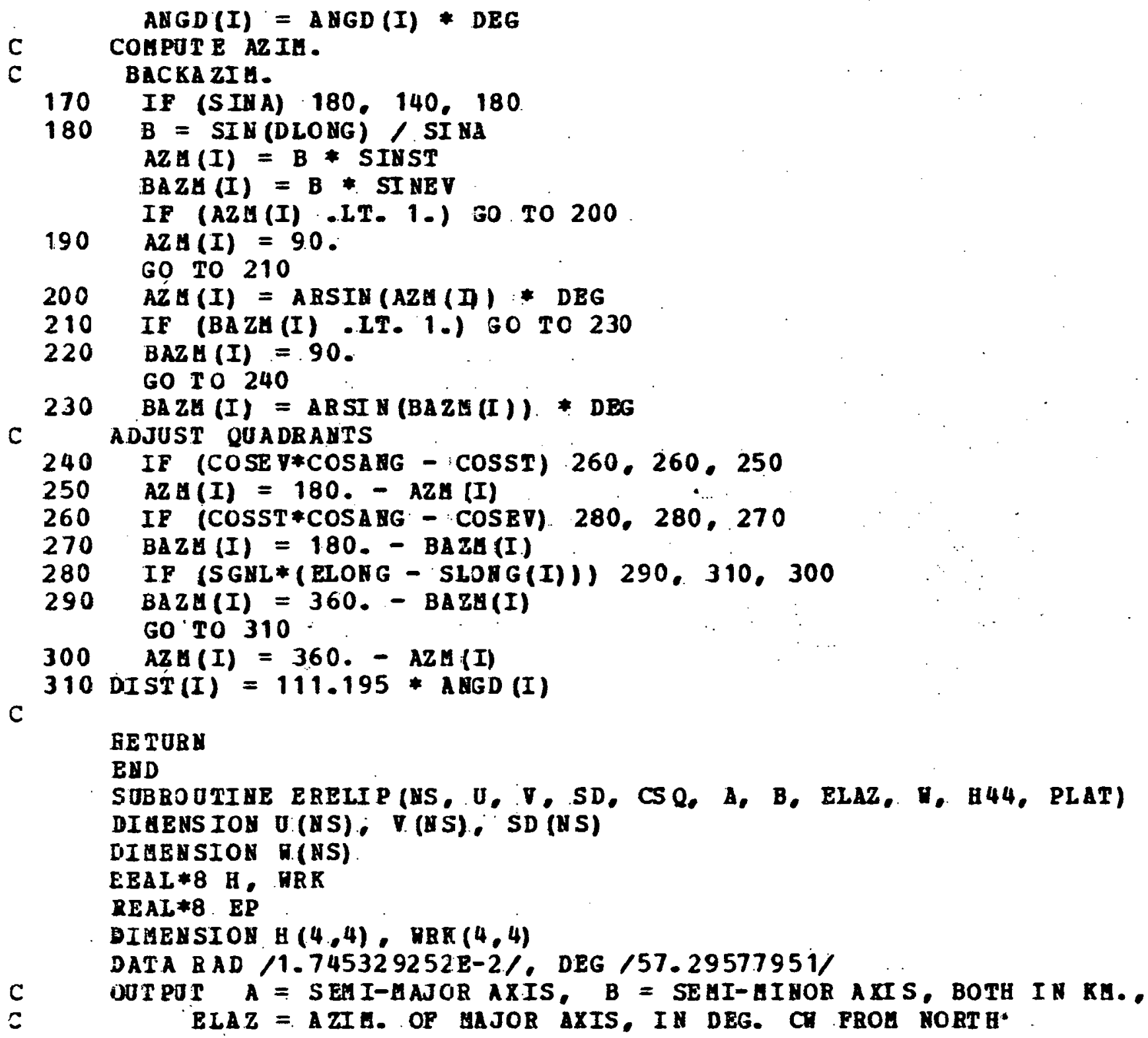




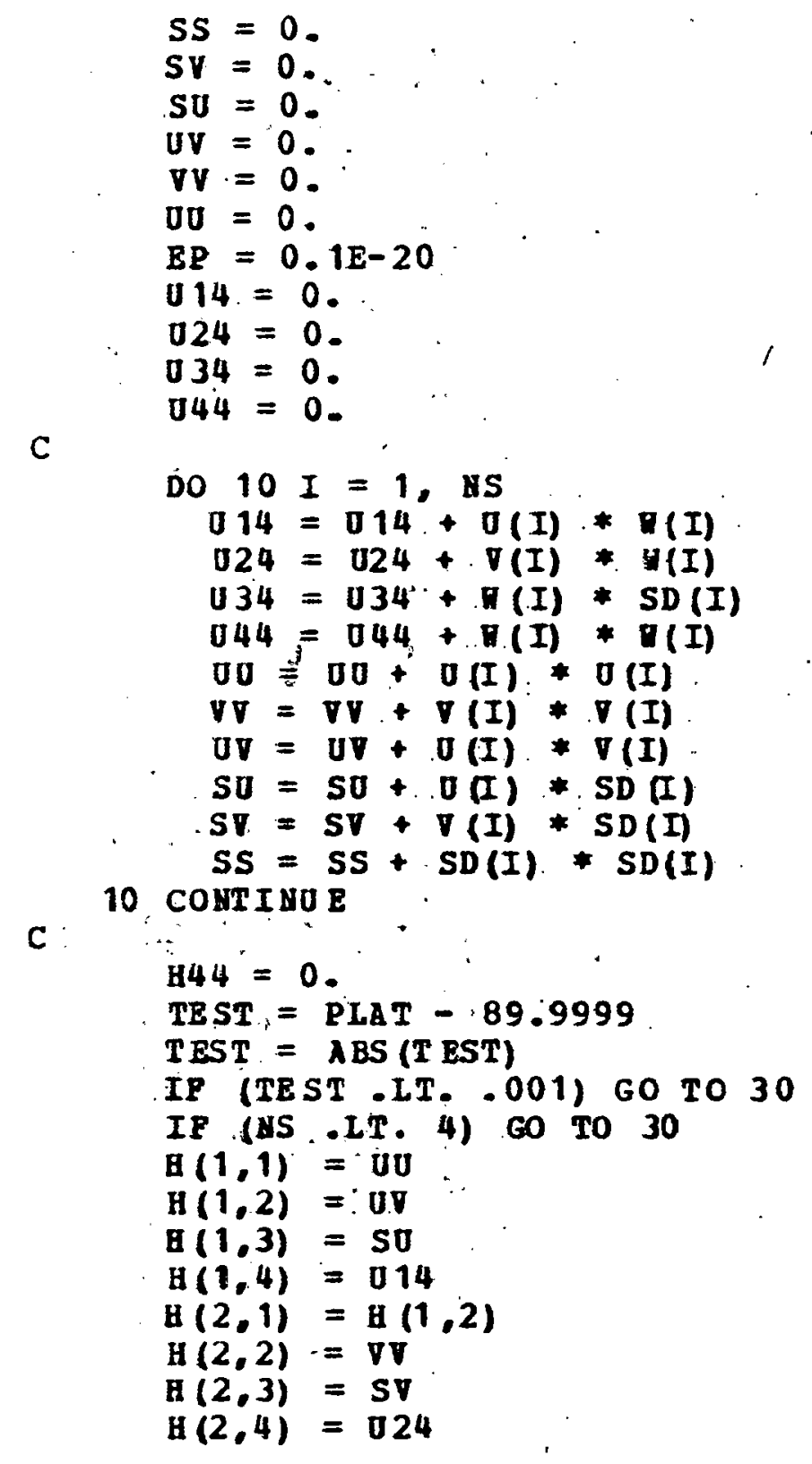




$$
\begin{aligned}
& \mathbf{B}(3,1)=\mathrm{B}(1 ; 3) \\
& \mathrm{B}(3,2)=\mathrm{B}(2,3) \\
& \mathrm{B}(3,3)=\text { SS } \\
& \text { B }(3,4):=034 \\
& \mathrm{H}(4,1)=\mathrm{B}(1 ; 4) \\
& \mathrm{B}(4,2)=\mathrm{B}(2,4) \\
& \mathrm{B}(4,3)=\mathrm{H}(3,4) \\
& \text { B }(4,4)=044 \\
& 84=4
\end{aligned}
$$

CALL GAUSS3 (14, EP, H, NBK, KER)

IF (KER - VE — 2) GO TO 20

G BITE $(6,180)$

GO TO 30

20 COUTIBU E

B $44=$ TBK (4, 4)

IF (B44.IT. 0.) $844=0$.

30 COVTI UB

$S S=1 . / S S$

$\nabla 0=0 U-\because S S * S U * S 0$

$V V=V \nabla-S S * S V * S V$

$U \nabla=2 . *(U \nabla-S S * S U * S \nabla)$

DOT $=0 \mathrm{U}-\mathrm{VV}$

IF (DUV) $50,40,50$

40 ALPB $=45$. RAD

GO TO 60

50 ALPB $=.5 *$ ATAN (UV/DOT)

$60 \operatorname{cosA}=\cos (A L P A)$

SINA = SIN (ALPH)

ALPH $=$ ALPH $* D E G$

SC UV $=$ UV * SIRA $*$ COSA

$\cos A=\cos A * \cos A$

SINA = SINA * SINA

$A=00 * \cos A+\nabla \nabla * \sin A+\operatorname{ScuV}$

$B=U U * S I N A+V V * \cos A-S \operatorname{CUV}$

IF $(B .-A) 70,80,90$

$70 \mathrm{DX}=\mathrm{A}$

$\mathrm{A}=\mathrm{B}$.

$B=D X$ 


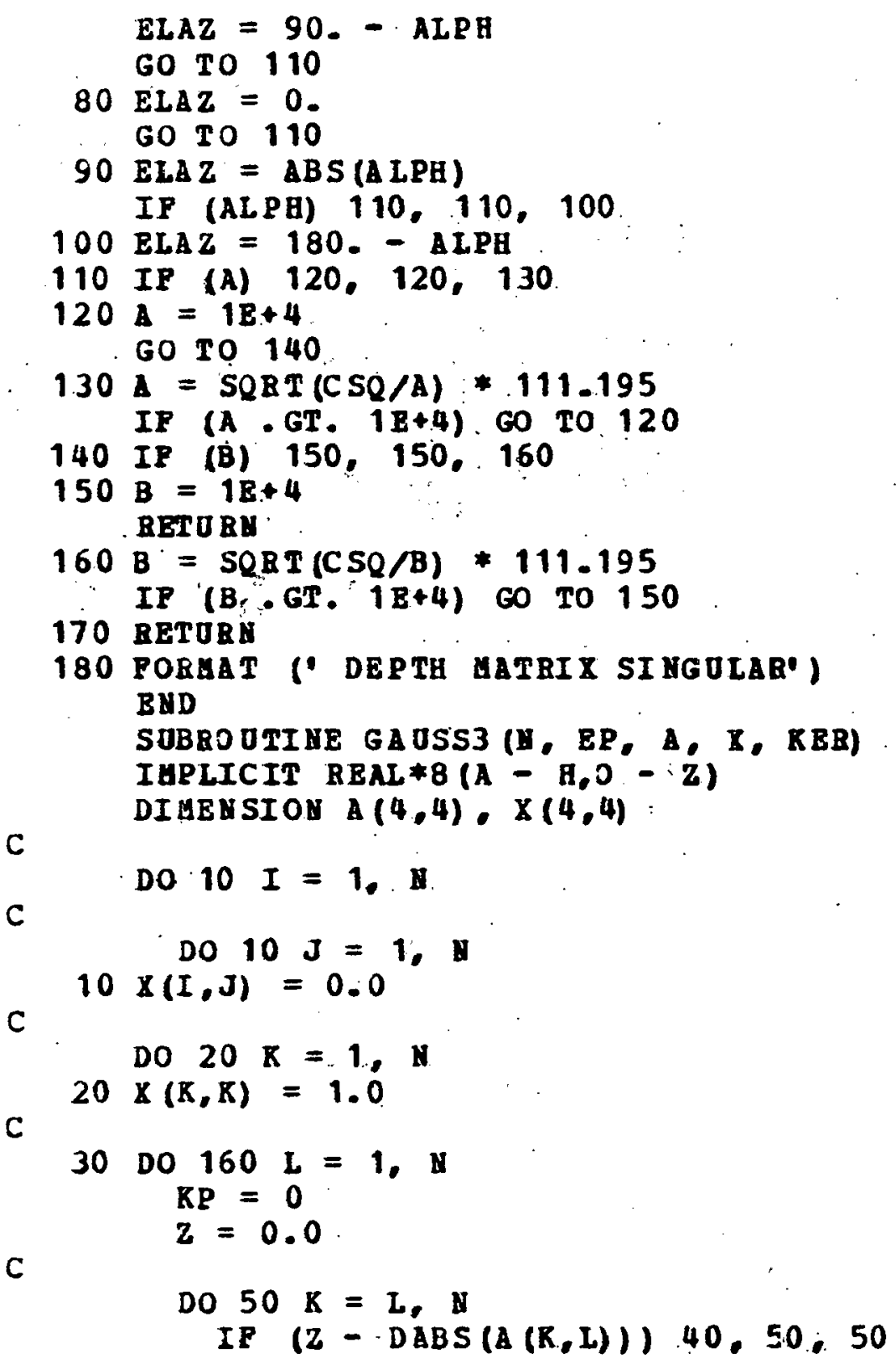




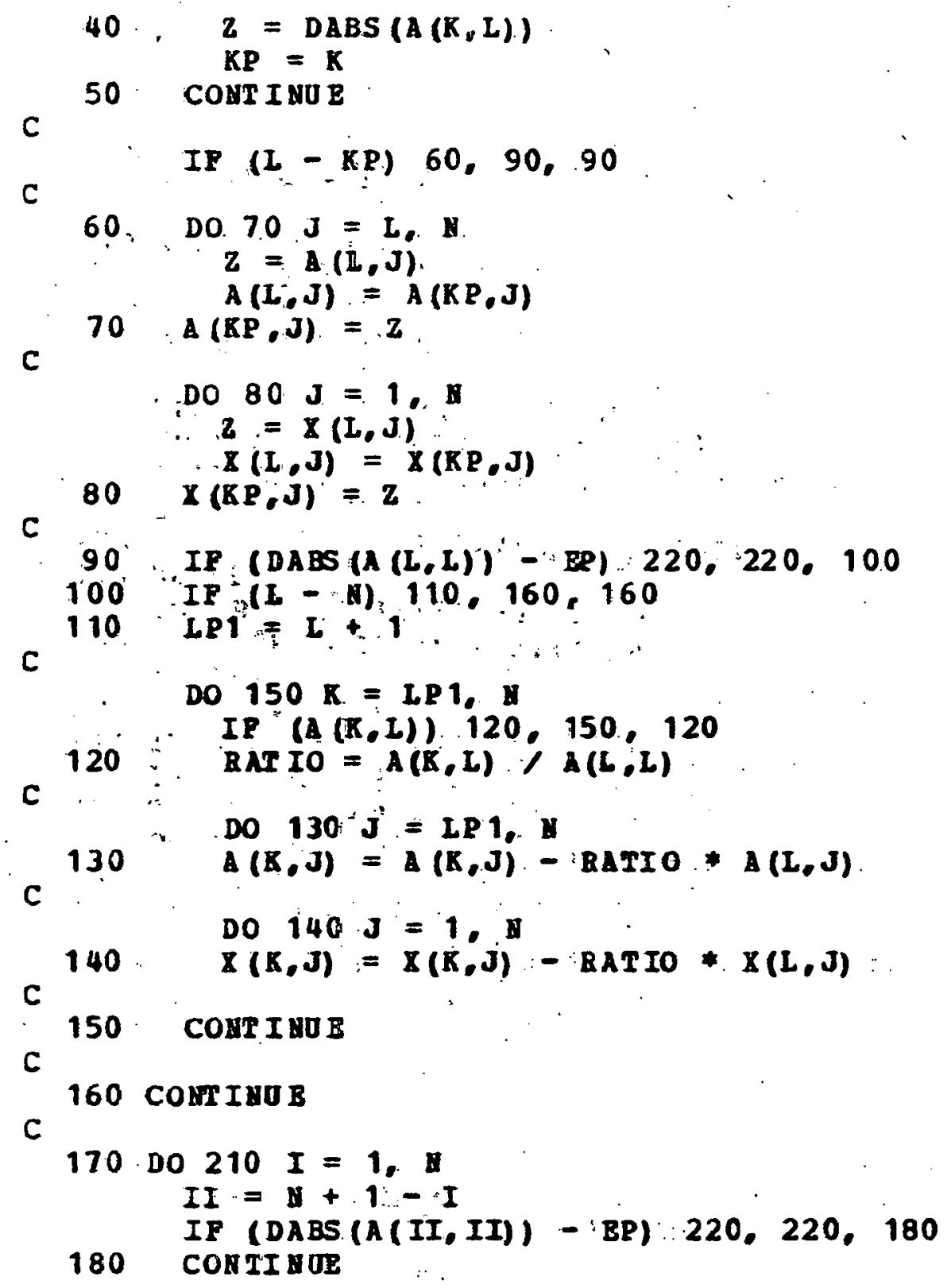


c

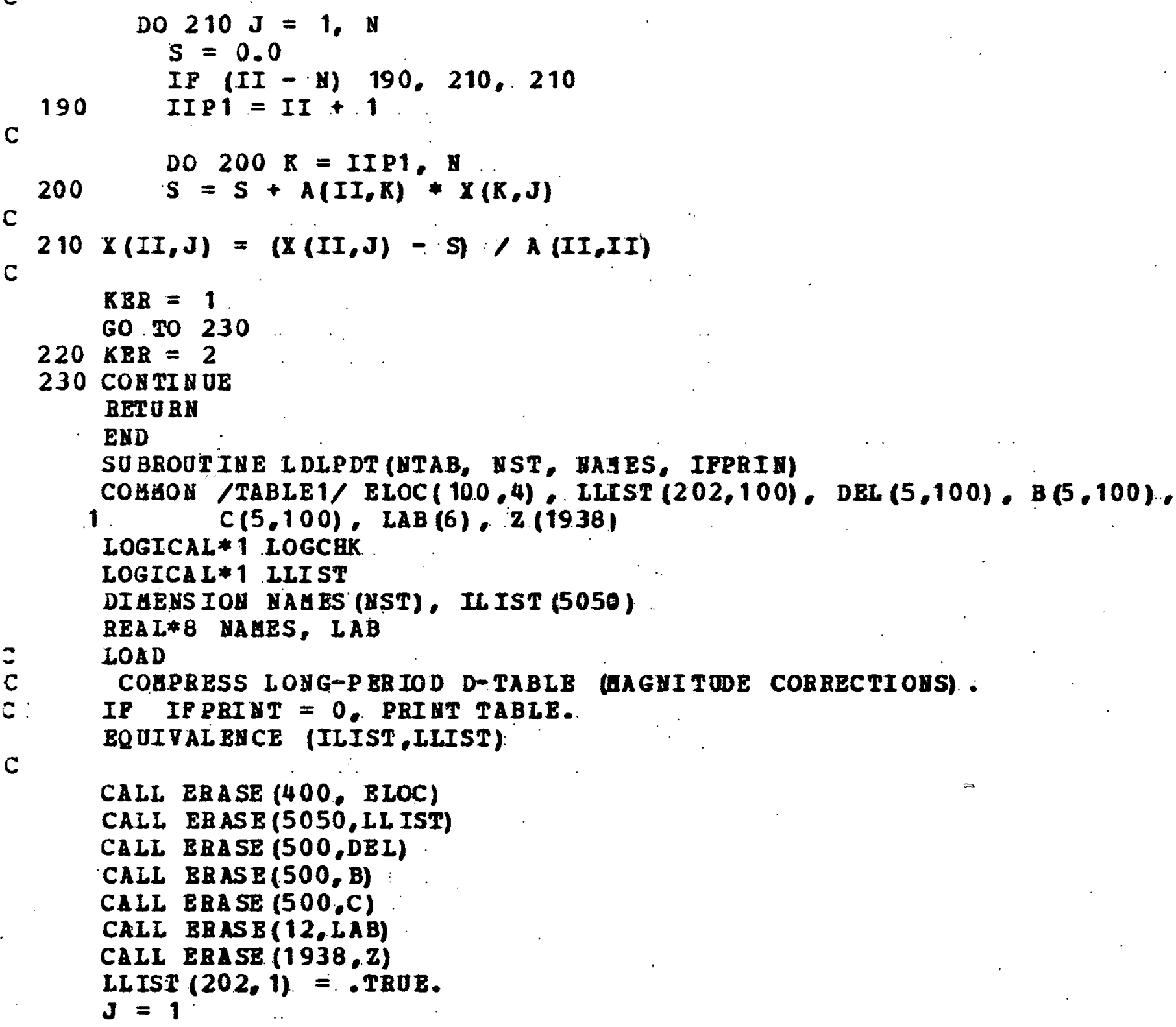




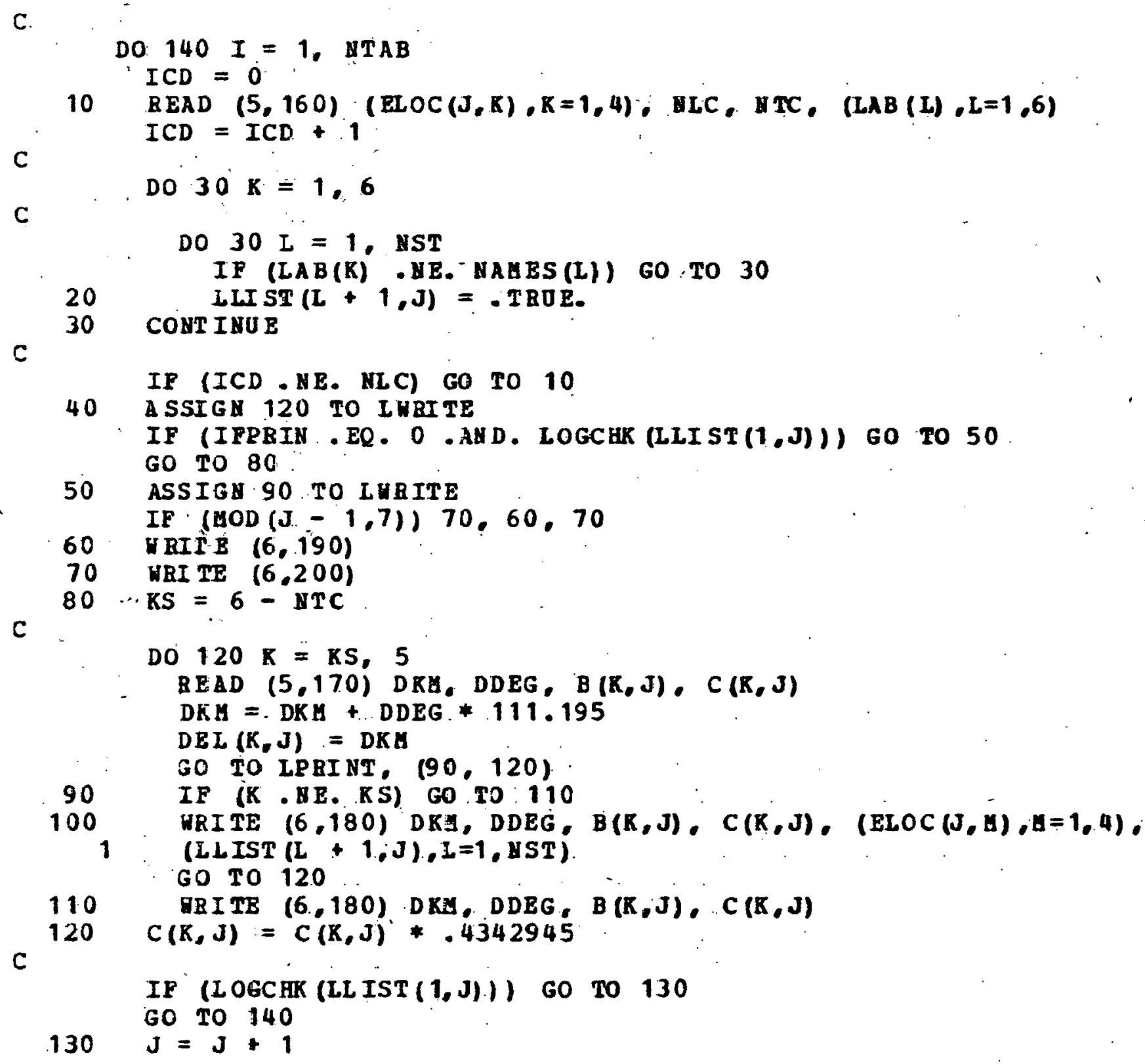




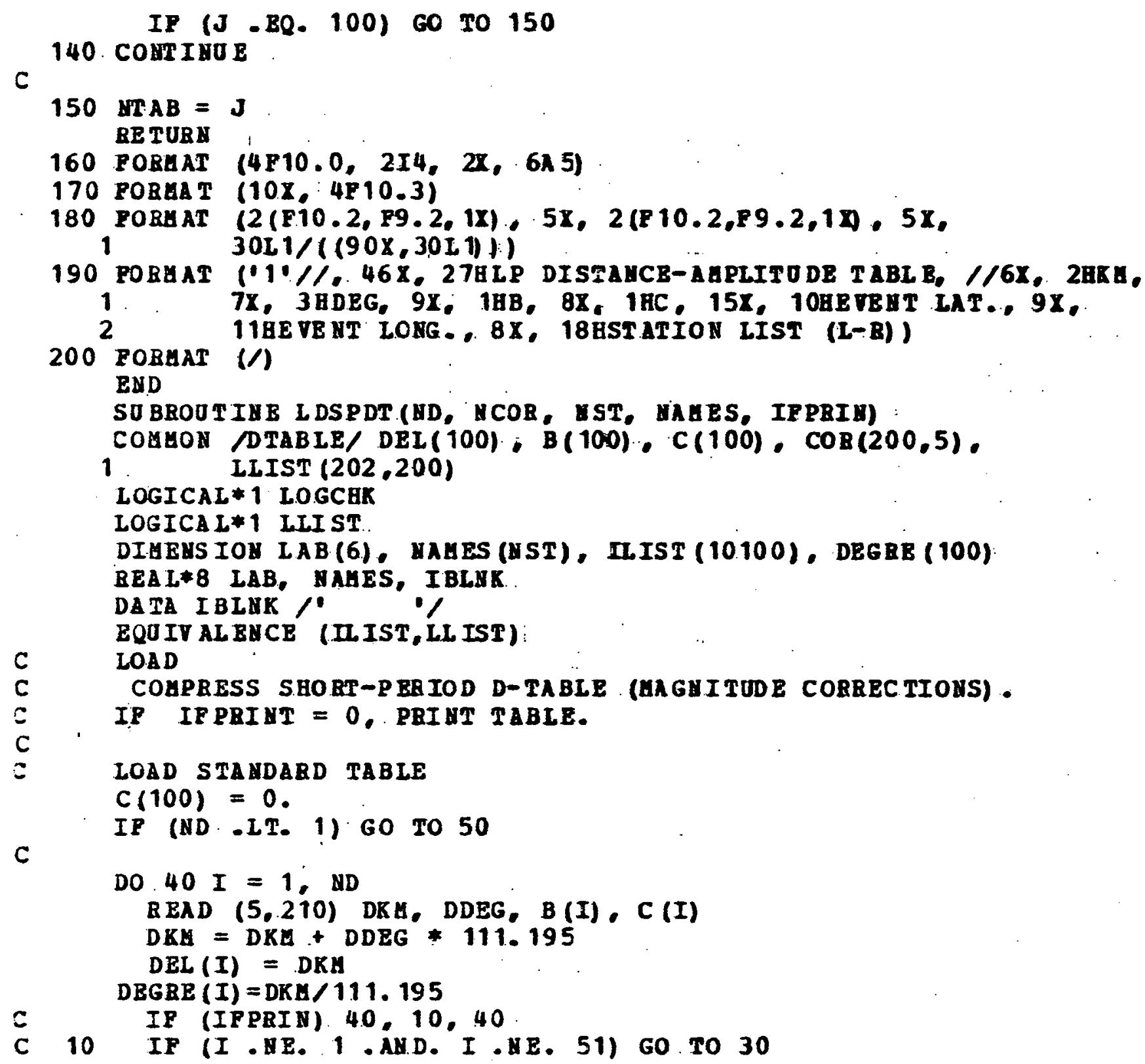




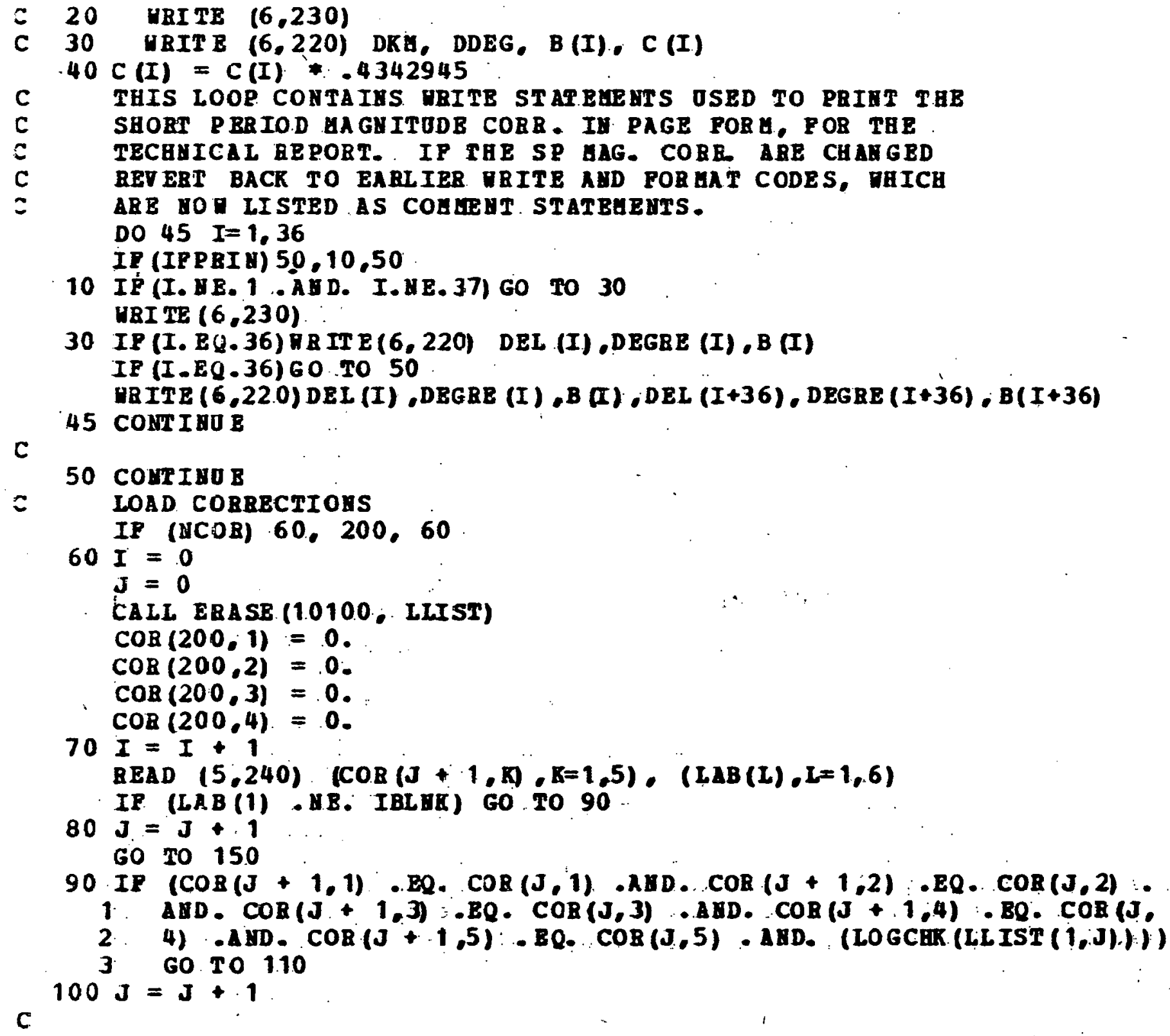


C

110 DO $130 \mathrm{~K}=1,6$

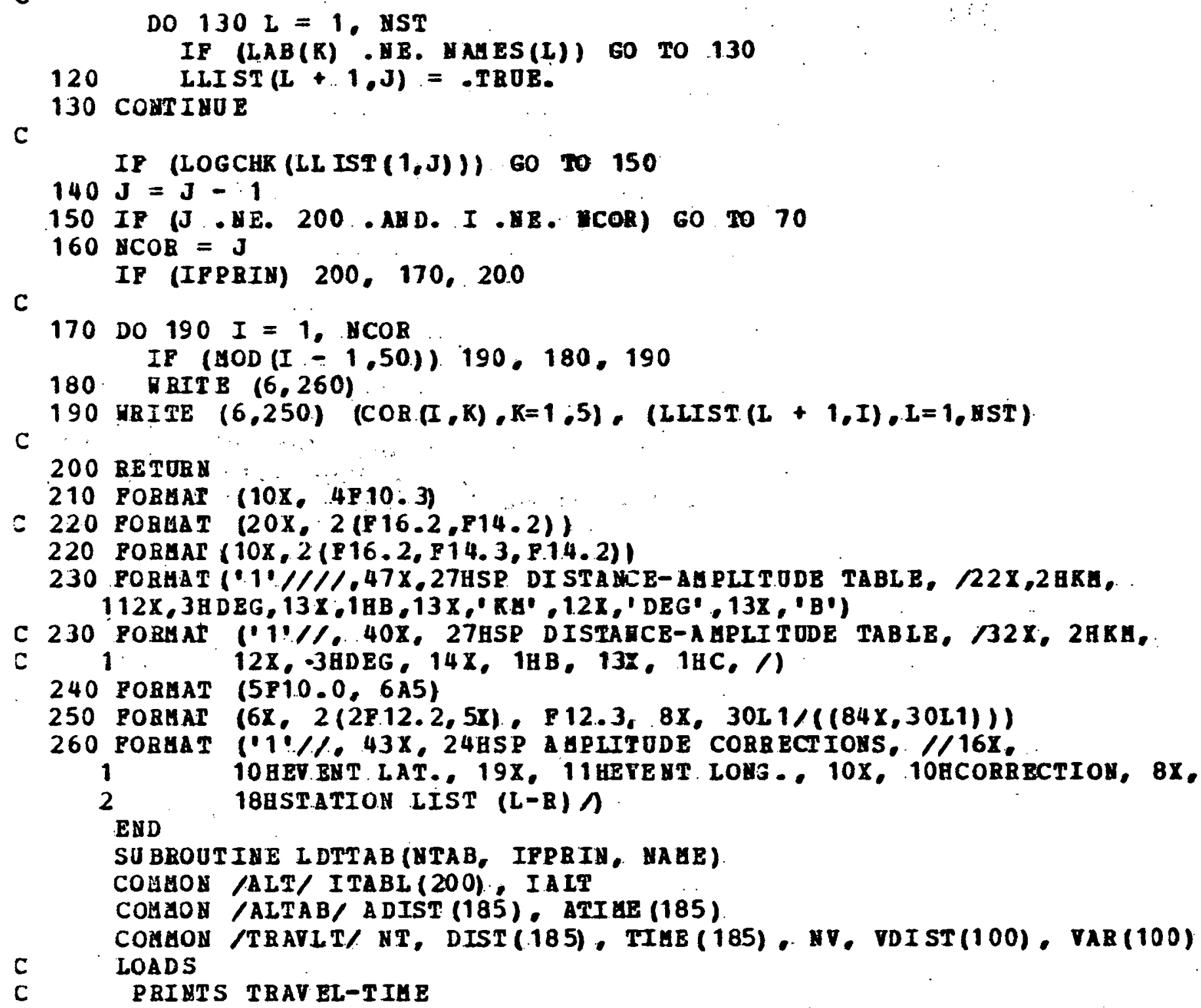

C PRINTS TRAV BL-TIEE 


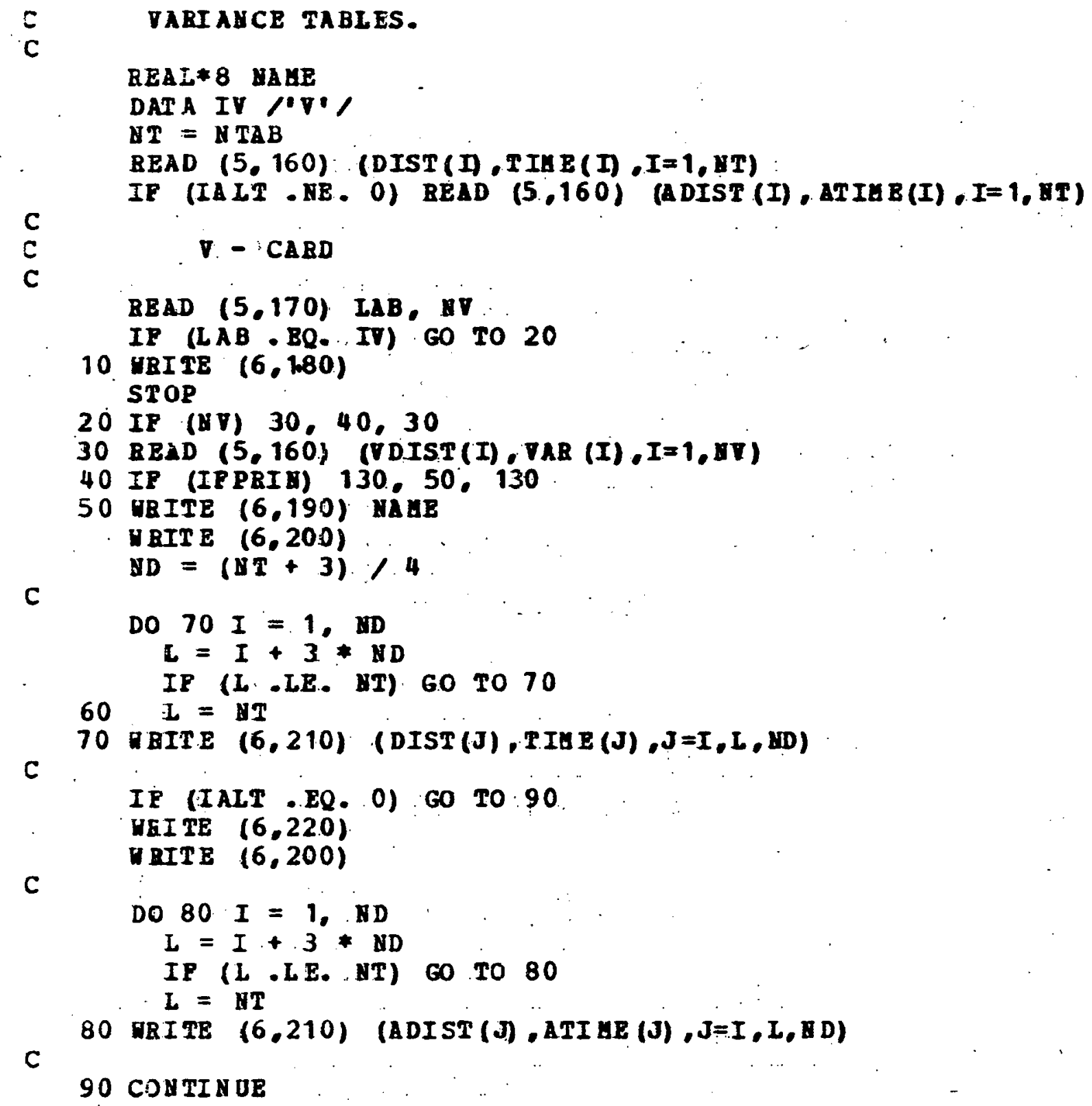




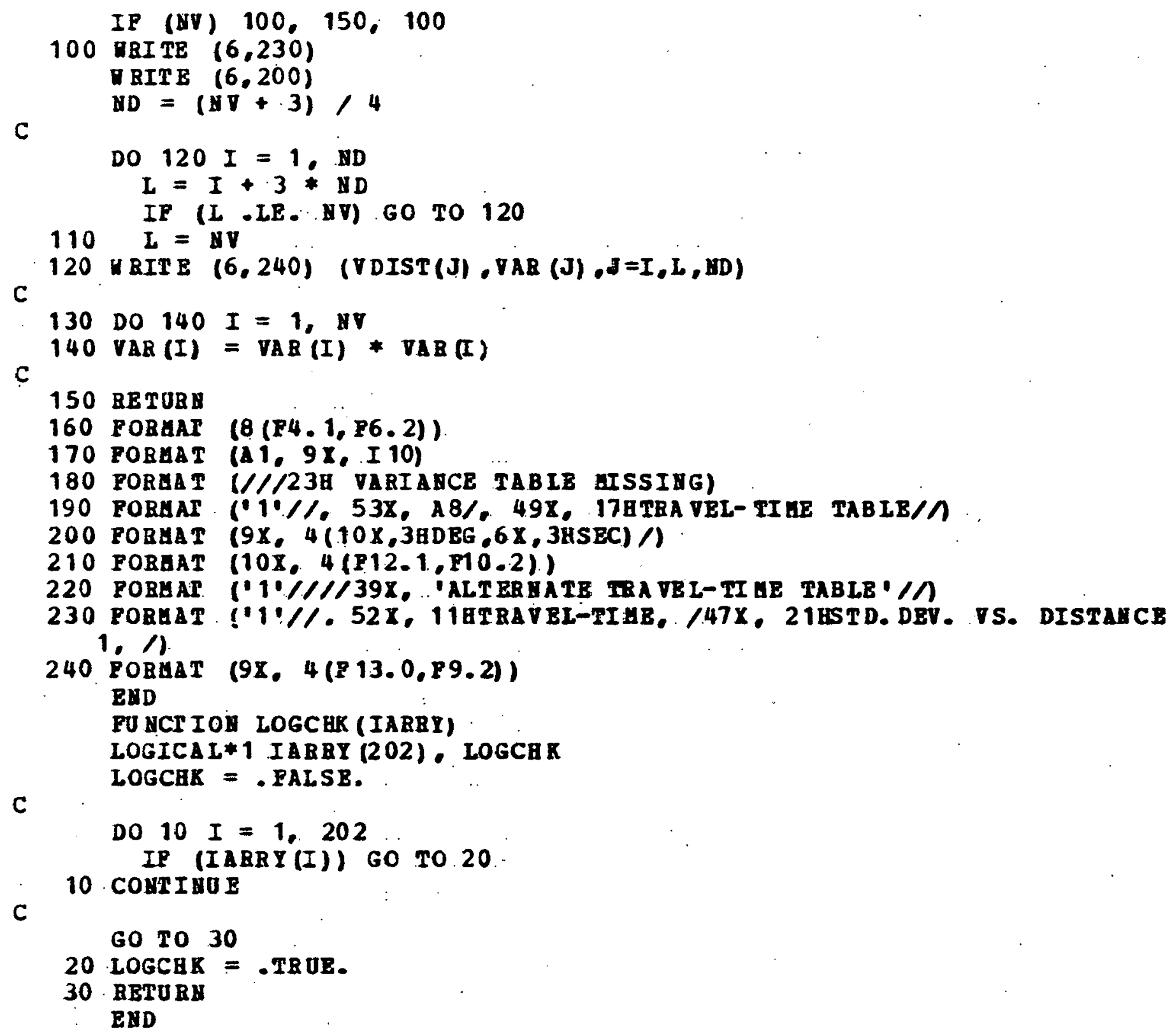


SUBROUTINE L PAAG (NST, DIST, SHAG; NTAB, FHAG, ELONG, ELAT)

COAMON /TABLE1/. CLATM (100) , ClaTP(10.0), CLONGH (100), CLONGP (100):

1 iLIST $(202,100)$, DEL $(5,100), B(5,100), C(5,100)$, IST $(200)$.

$2 \quad 2(1750)$

LOGICAL*1 LLIST

DIMENS IOA DIST (NST) , SHAG (AST)

- DOES LONG-PERIOD MAGNITUDE COBRECTIONS.

C OOTPUT SMAG = MAGNITUDES AS RECVD BY EACH STATION.

DO $10 \cdot I=1, N S T$.

C

$10 I S T(I)=1$

C

IF (NTAB .LT. 2) GO TC 60

DO $50 I=2$, NTAB

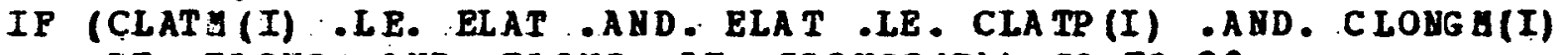
LE. ELONG .AND. ELONG - LE. CLONGP(I)) GO TO 20

c GO TO 50

20 DO $40 \mathrm{~J}=1$, NST IF (LLIST $(J+1, I)$ Go TO 30

$30 \quad$ GO TO 40 :

C

40 CONTINUE

50 CJNTIN TJE

C

60 DO $160 \mathrm{~J}=1$, NST

C $\mathbf{J} \mathbf{J}=\mathbf{I S T}(\mathbf{J})$

c

DO $70 . I=1,5$

70 CONTINUE

$80 \quad I=5$

$80 \operatorname{SHAG}(\mathrm{J})=F M A G+B(I, J J)$

IF (C(I,JJ)) 90. 110.90

SO IF (DIST(J). . LE. 1.) GO TO 160 


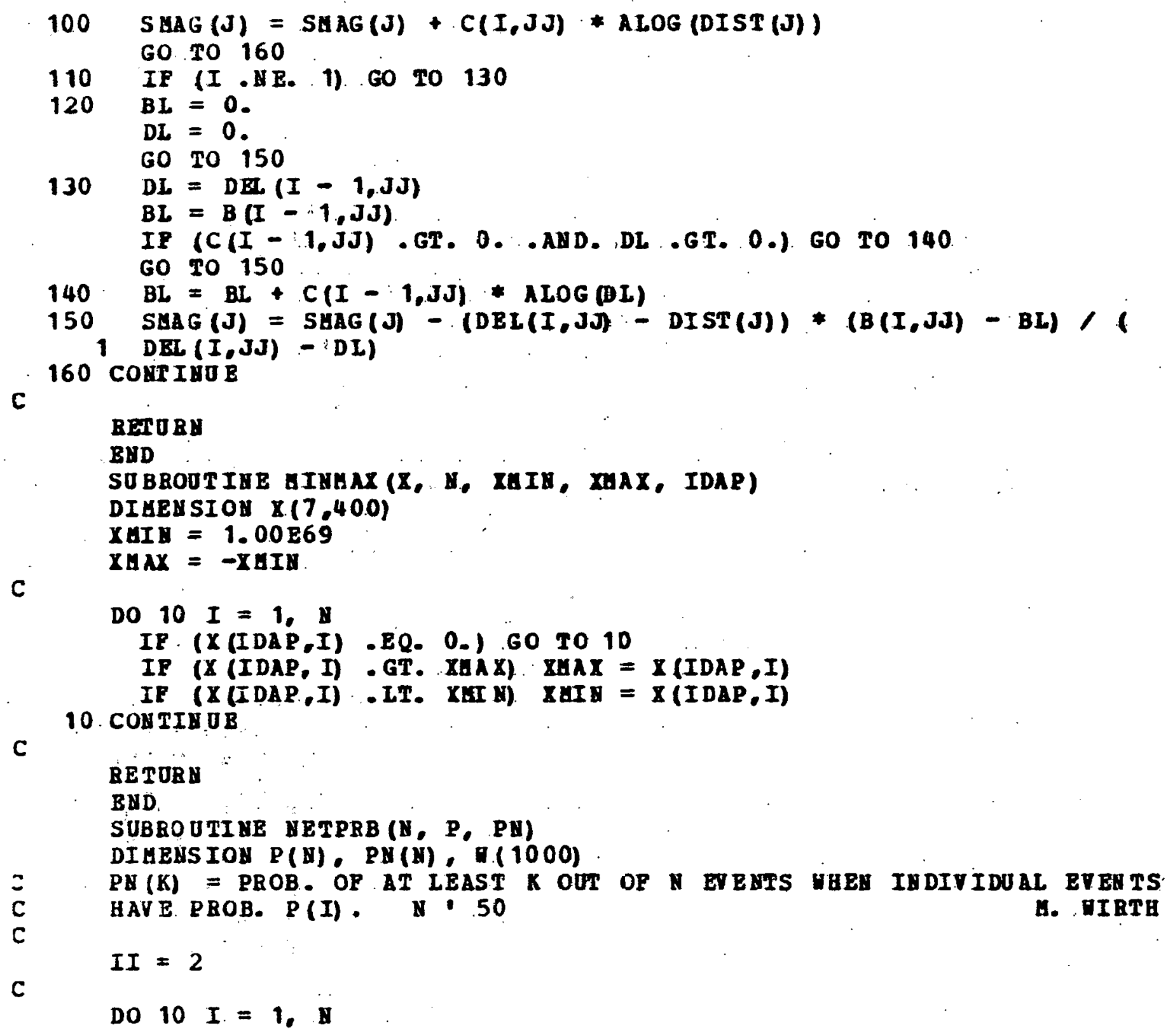




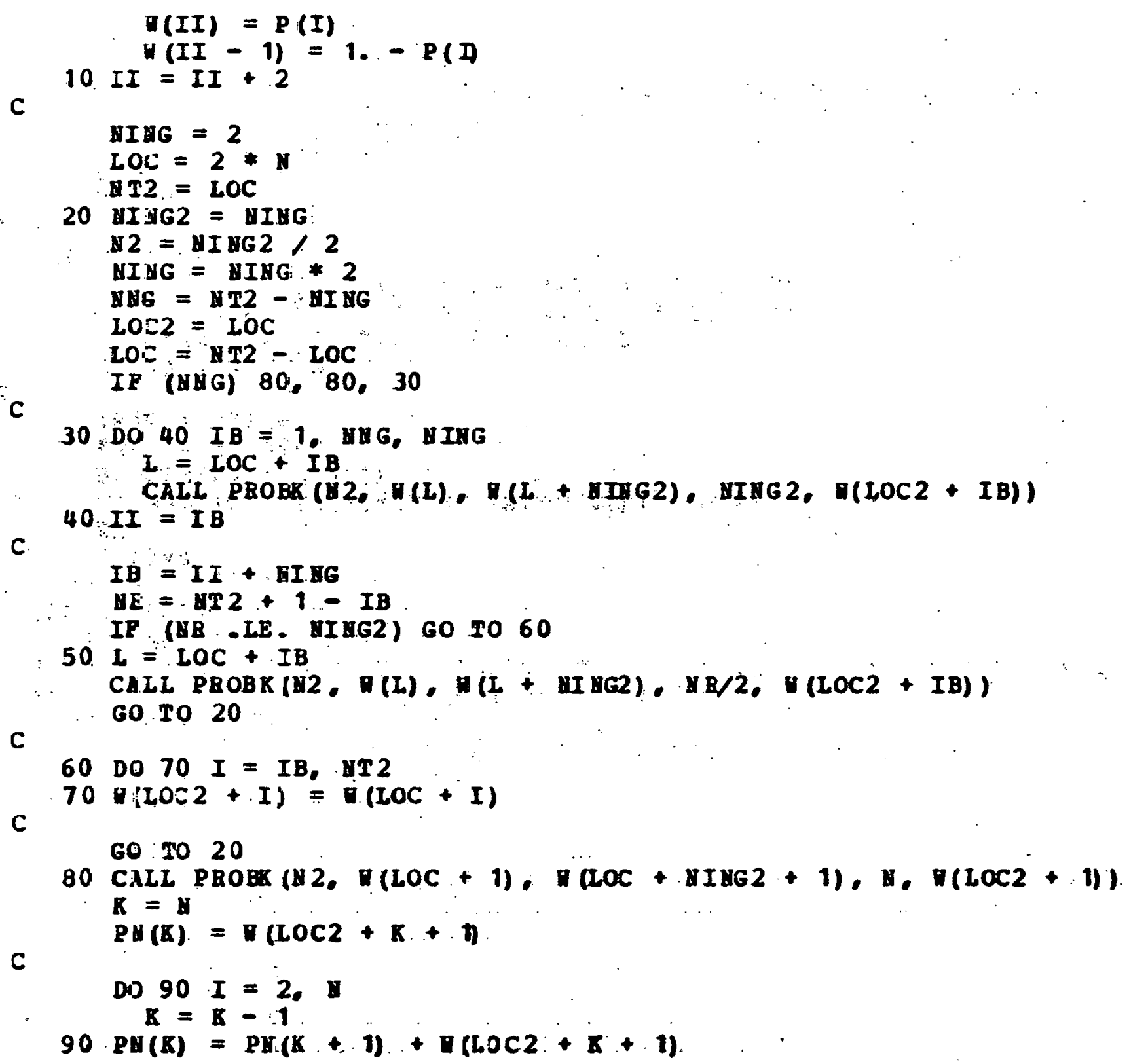


C RETURN

END

SOBRO UTI UE ORDREI (N, $X, I)$

DI HEHS ION $X(N)$. I (B)

* ORDER * $X *$. LARGEST TO SHALLEST, AMD REUBABBR ITS SOBSCBIPTS * I*

DO $10 \mathrm{~J}=1, \mathrm{~J}$

$c$

$10 I(J)=J$

$$
\text { DO } \begin{aligned}
40 J & =1, Y \\
\text { XEAX } & =X(J) \\
\text { IUAX } & =J
\end{aligned}
$$

C

DO $30 \mathrm{X}=\mathrm{J}, \mathrm{H}$

$20 \quad X B A X=X(X)$.

LABX $=\mathbf{K}$

30 CONT I RU B

C

$$
X(\operatorname{LAX})=X(\mathrm{~J})
$$

$K=I(\operatorname{LHAX})$

$I(\operatorname{LaAX})=I(J)$

$I(J)=K$

$c$

$40 X(J)=X \operatorname{MAX}$

\section{BETU RE}

END

FU UCTIOU PROBF (X)

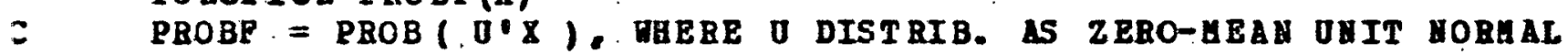
DOO BLE PRECISION $Q$. T

$T=\operatorname{ABS}(\mathrm{X})$

$Q=(((T * .00063419-.00010754) * T+.01057706) * T+.04833145) * T+$

$1.10882473) * T+1.09050773$

$Q=1.0 /(Q * Q)$

$Q=Q * Q$

PROBP $=Q * Q$

IF (X .I.E. 0.$)$ GO TO 20

$10 \mathrm{PROBF}=1 .-\mathrm{PROBP}$ 


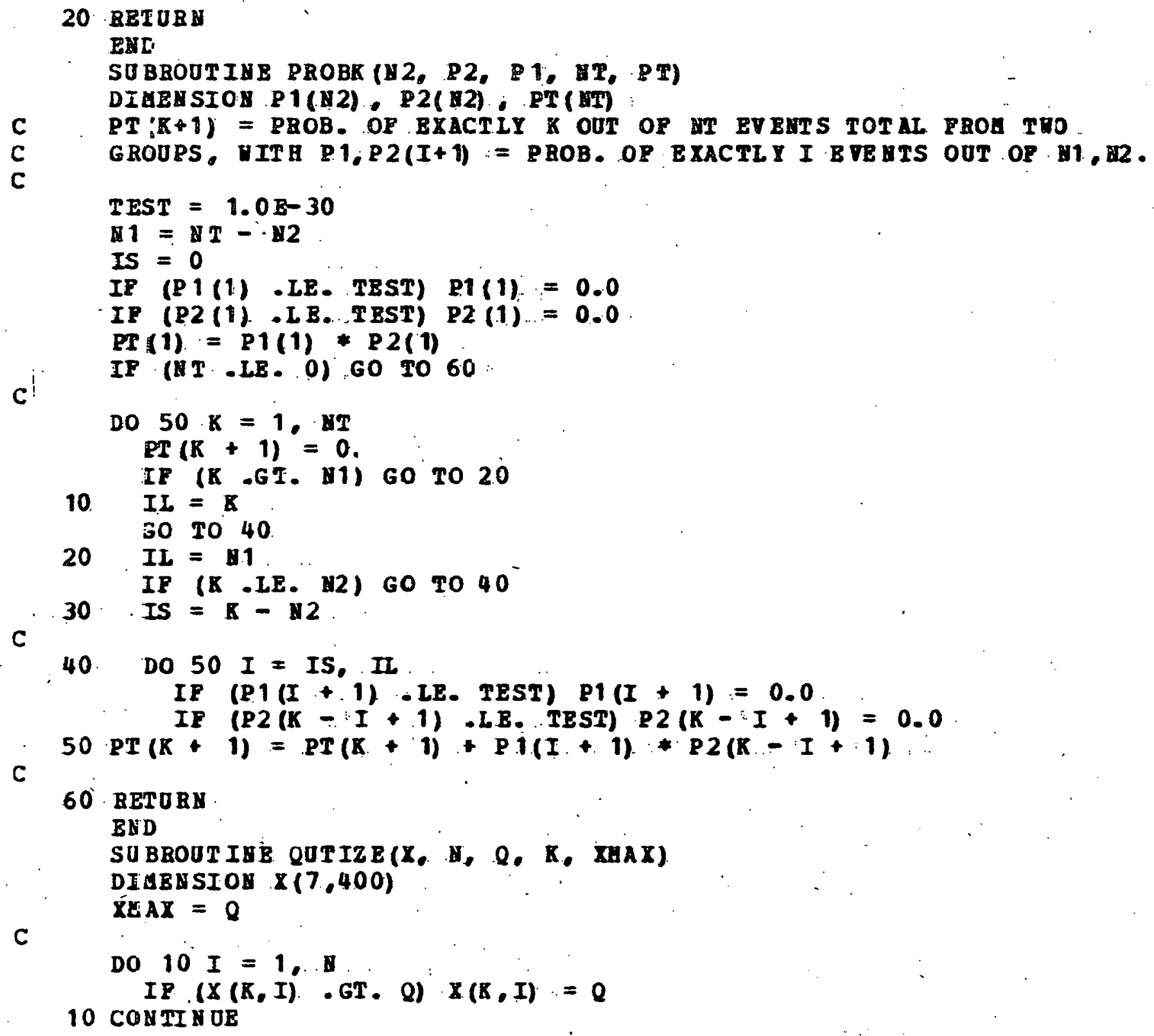


c

RETURI

ËDD

SU BROUT INE SPMAG (NST, SCOB, DIST, SHAG, MCOR, HD, FHAG, BLOMg, 1 ELA T)

LOGICAL $* 1$ LOGCRK

COBBOA /DTABLE/ DEL(100), B(100), C(100), CLara (200), CLATP (200).

1

LOGICAL*1 IL IST

DIUE ASION SCOR (AST), DIST (NST), S AAG (BST), ILIST (10100)

BQUIV ALENCE (IIIST, LL IST)

C DOES SHORT-PERIOD HAGIT TUDE CORRECTIONS.

INPOT HST = NO: STATIONS, SCOR = ST AT. COBRBCTIONS, BTC.

OUTPUT SEAG = HAGITTODES AS BECVD BY EACH STATIOA

CALT BEASE (IST, SAAG)

EVCOR $=0$.

IFES $=0$

IFEV $=0$

IP (HCOR, IT. 1) 60 TO 80

c

DO $70 I=1$, ICOR

IP (CLATE (I) .IE. ELAT A AD. ELAT .IE. CLATP(I) . AND. CLORGE(I)

1 - LB. BLOHG AND. BLOHG IB. CLOYGP(I)) GO TO 20.

10 IF (IPES . EQ. 1 . AN D. IPBV .EQ. 1) GO TO 80

GO TO 70

20 IF (LOGCBK (LLIST (I; D)). GO T0 40

30 IFEV $=1$

$\mathrm{ETCOB}=\operatorname{COR}(\mathrm{I})$

GO TO 70

40 IPES $=1$

C

DO $60 \mathrm{~J}=1$. UST

IP (LISTT $(J+1 . I)) 60$ TO 50

60 TO 60

$50 \quad \operatorname{SHAG}(J)=\operatorname{COR}(I)$

c

60 COETINOE 
c

c

70 CDNTINOE

c

$\cdots$

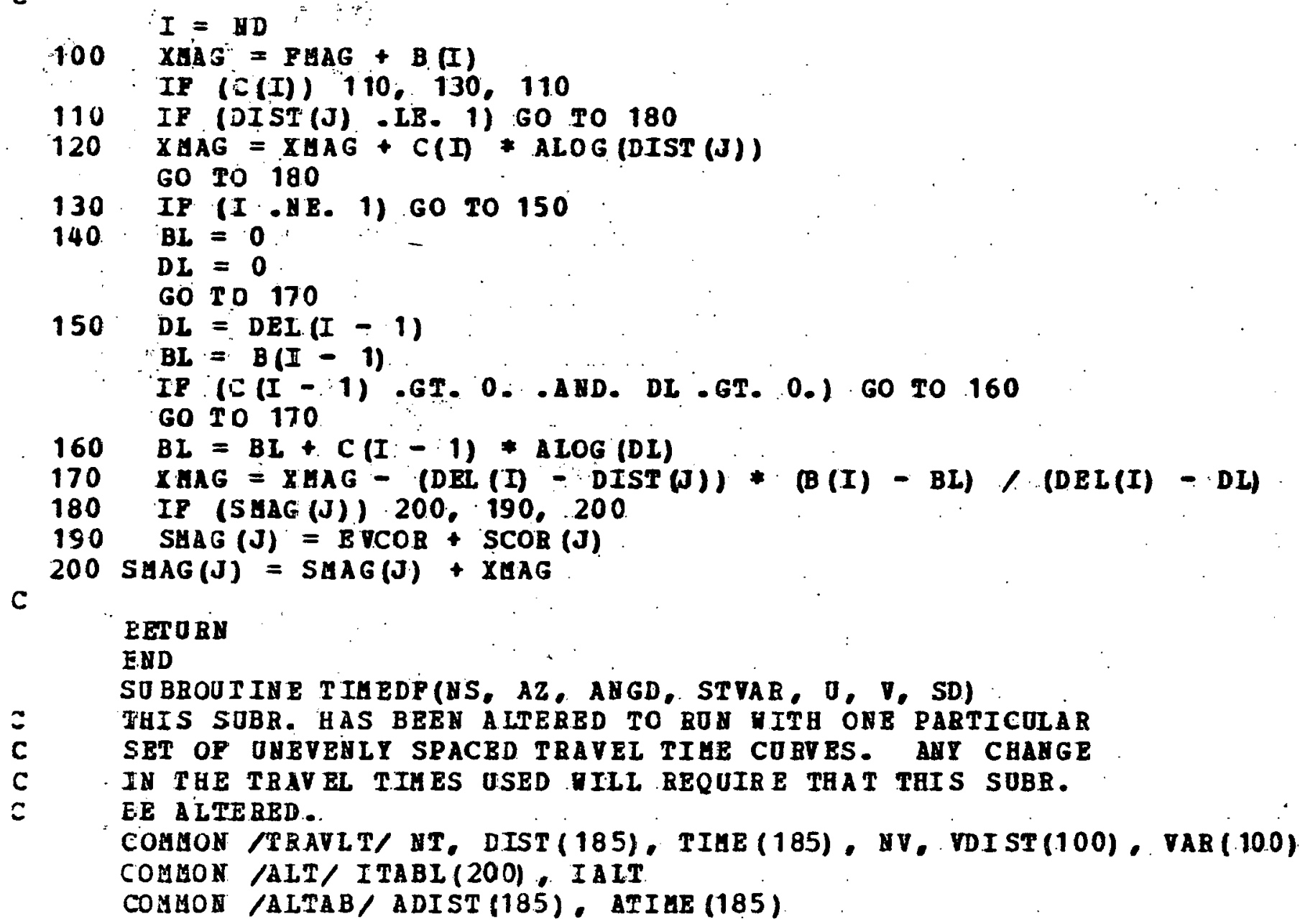




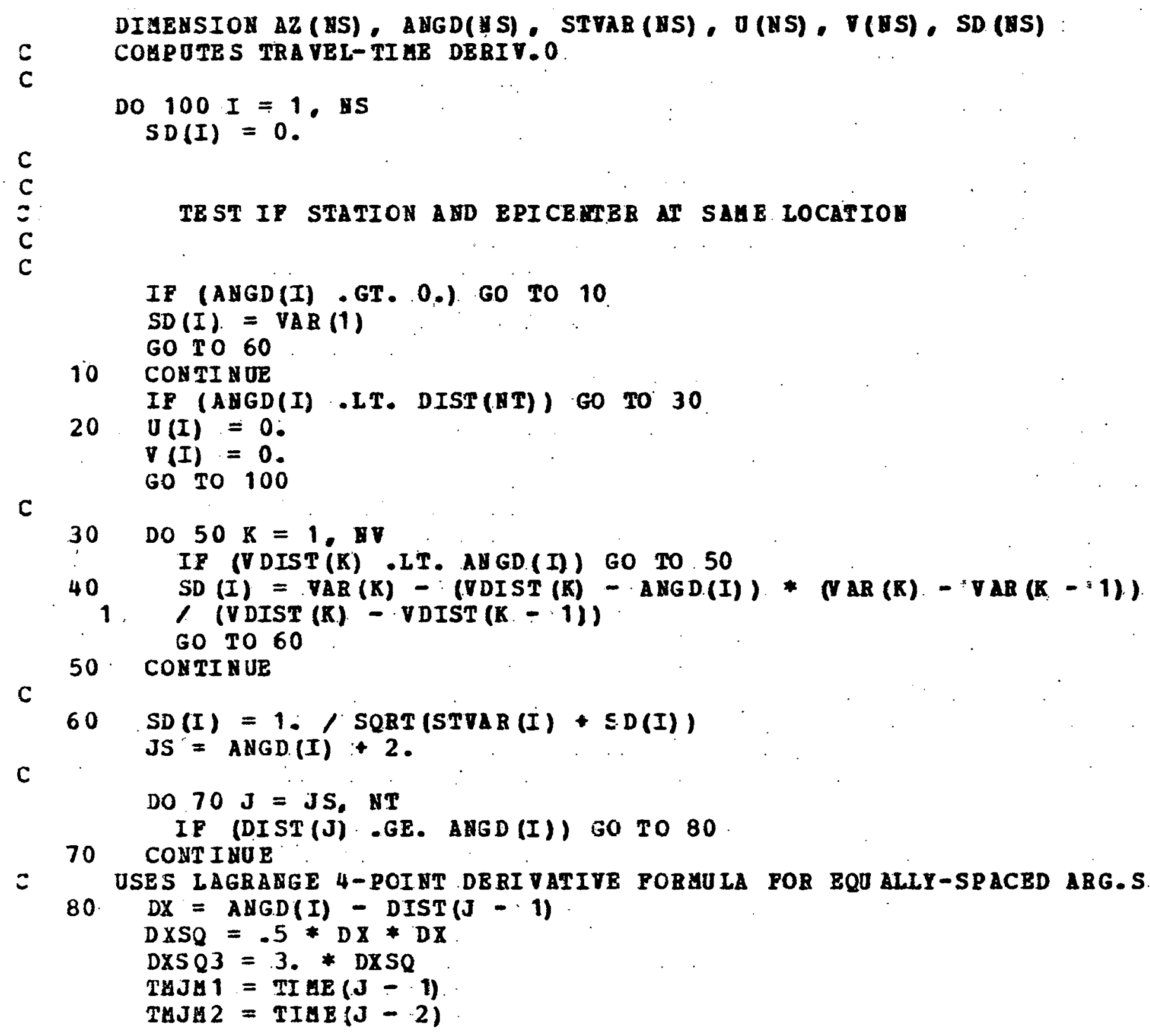


C

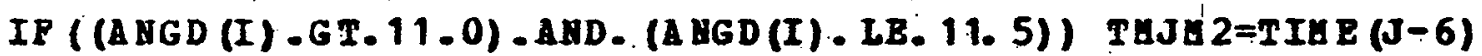

\section{RE TORA}

BND

SUBRO TTIEE TI UET (HS, AUGD, B)

C. THIS SUBROUTIBB BAS BEBD ALTEBED TO RUD IITH OAE

C PARTICULAR SET OF OHE YBHLI SPACED TRAVEL TIBE

C CURVBS. AHY. CHAMGB IN THE TRAVEL TIGES OSED

¿. IILL RBQUTRE TEAT THIS SUBB. BB ALTBRED.

COHEON/ALT/. ITABL $(200)$. IALT 
COUBON /ALTAB/ ADIST (185), ATIAB (185)

COHKON /TBAVLT/ HT, DIST (185), TIME (185)

C

DI HEYSION ANGD (XS), R (NS)

DO $60 I=1$, NS

IF (ANGD(I) ILT. DIST (XT)) GO TO 20

$10 \quad B(I)=0$.

GO TO 60 .

C

$20 \quad \mathrm{JS}=\mathrm{AUGD}(I)+2$.

DO $30 \mathrm{~J}=\mathrm{JS}, \mathrm{NT}$

IF (DIST (J) . GB. AHGD(I)) 60 TO 40

C

30 CONTI NUE

$40 D X=A H G D(I)-D I S T(J-1)$

$P=D X$

PU1 $=\mathbf{P}-1$

$\mathrm{PH} 2=\mathrm{P}-2$

PSH $1=P * P-1$

PP1 $=\mathbf{P}+1$

TUJU1 $=\operatorname{TI} U E(J-1)$

TAJA2 $=\operatorname{TIAB(J-2)}$

C

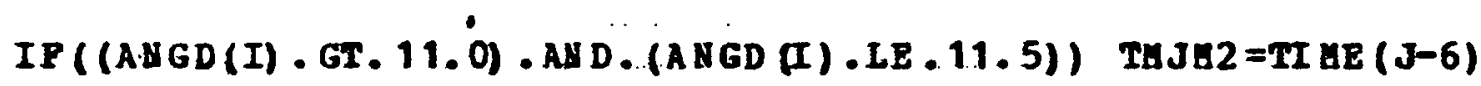

$\operatorname{TBJP} 1=\operatorname{TIMB}(J+1)$

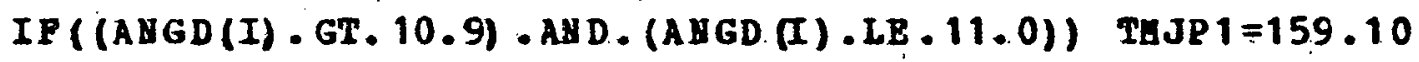

$T M J=T I H E(J)$

IP (I TABI (I) - EQ. 0): GO TO 50

THJHI $=\operatorname{ATIMB}(J-1)$

TUJष2 $=$ ATIME $(J-2)$

C

$\operatorname{IF}((A H G D(I) \cdot G T \cdot 11.0)$. ABD. (ANGD(I) - IE. 11.5)) THJU2=TIHE $(J-6)$

C

TUJP $1=\operatorname{ATI}$ UE $(J+1)$ 
c

IP( (ANGD(I) - GT.1 0.9) . AND. (A IGD (I) . LE. 11..0) ) TEJP 1=159. 10

c

50 TON $=A \operatorname{TILE}(\mathrm{J})$

$R(I)=-((P * P B 1 * P B 2) / 6) * T W H 2+.((P S B 1 * P B 2) / 2) * T H J B 1-.(1$

$1 \mathrm{P} * \mathrm{PR} 1 * \mathrm{PH} 2) / 2.) * \mathrm{THJ}+((\mathrm{P} * \mathrm{PSE} 1) / 6) *. T \mathrm{TJP} 1$

c

60 COMTIEOE

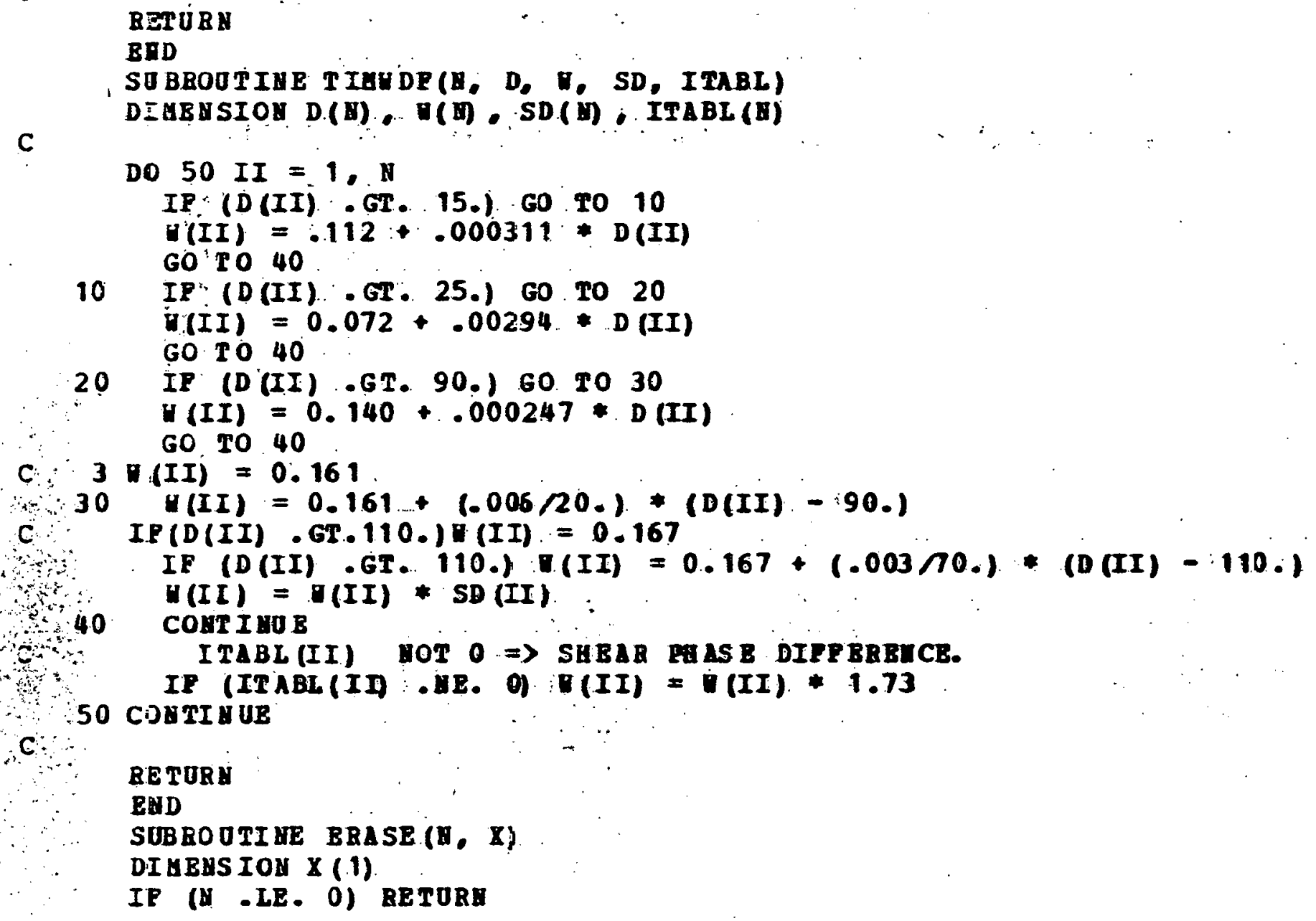


C

DO $10 I=1, y$

$X(I)=0$.

10 CONTINDE

c

BETURE

END 


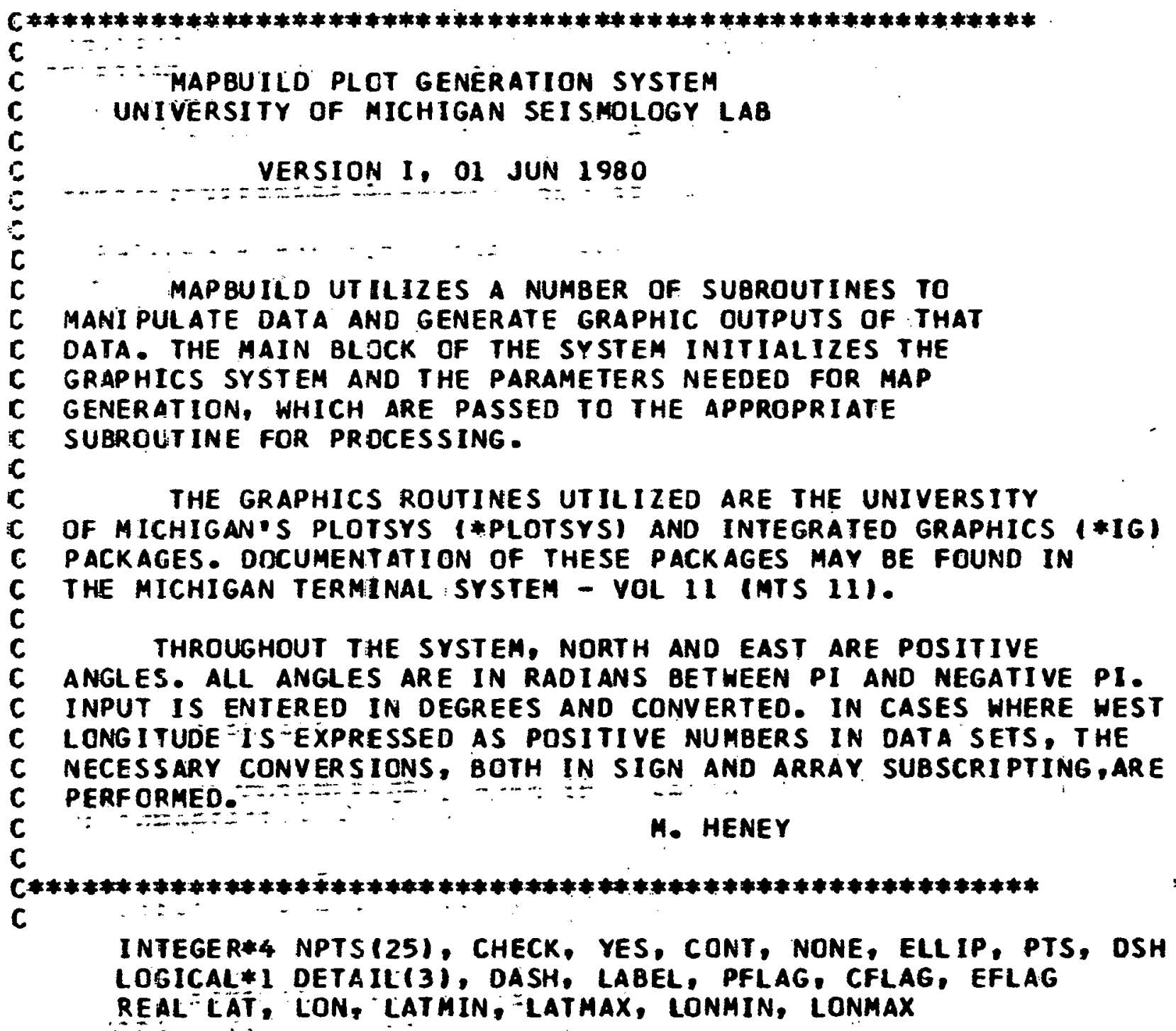

THROUGHOUT THE SYSTEM, NORTH AND EAST ARE POSITIVE ANGLES. ALL ANGLES ARE IN RADIANS BETWEEN PI AND NEGATIVE PI. INPUT IS ENTERED IN DEGREES AND CONYERTED. IN CASES WHERE WEST LONG ITUDE IS EXPRESSED AS POSITIVE NUMBERS IN DATA SETS, THE NECESSARY CONVERSIONS, BOTH IN SIGN AND ARRAY SUBSCRIPTING,ARE 


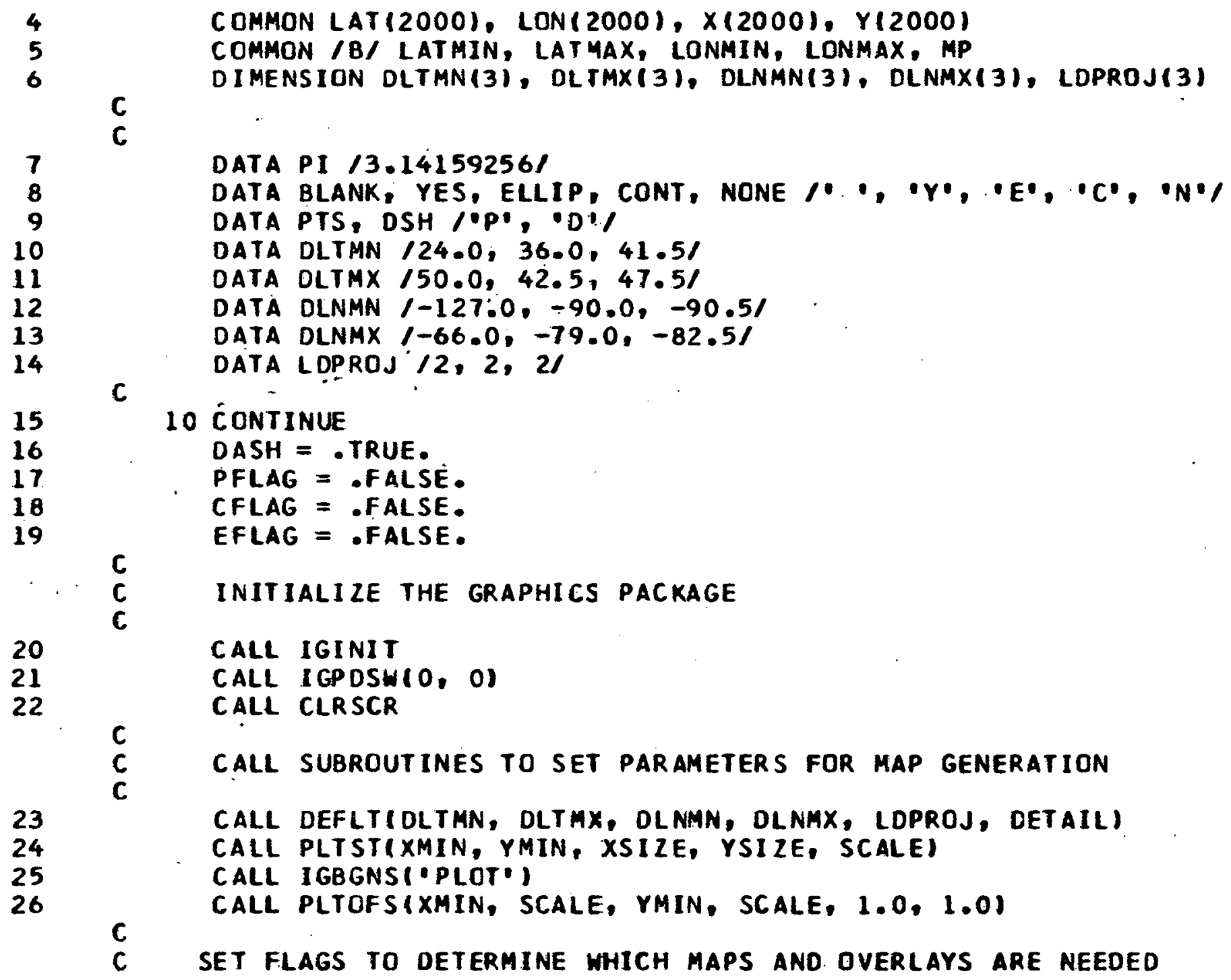




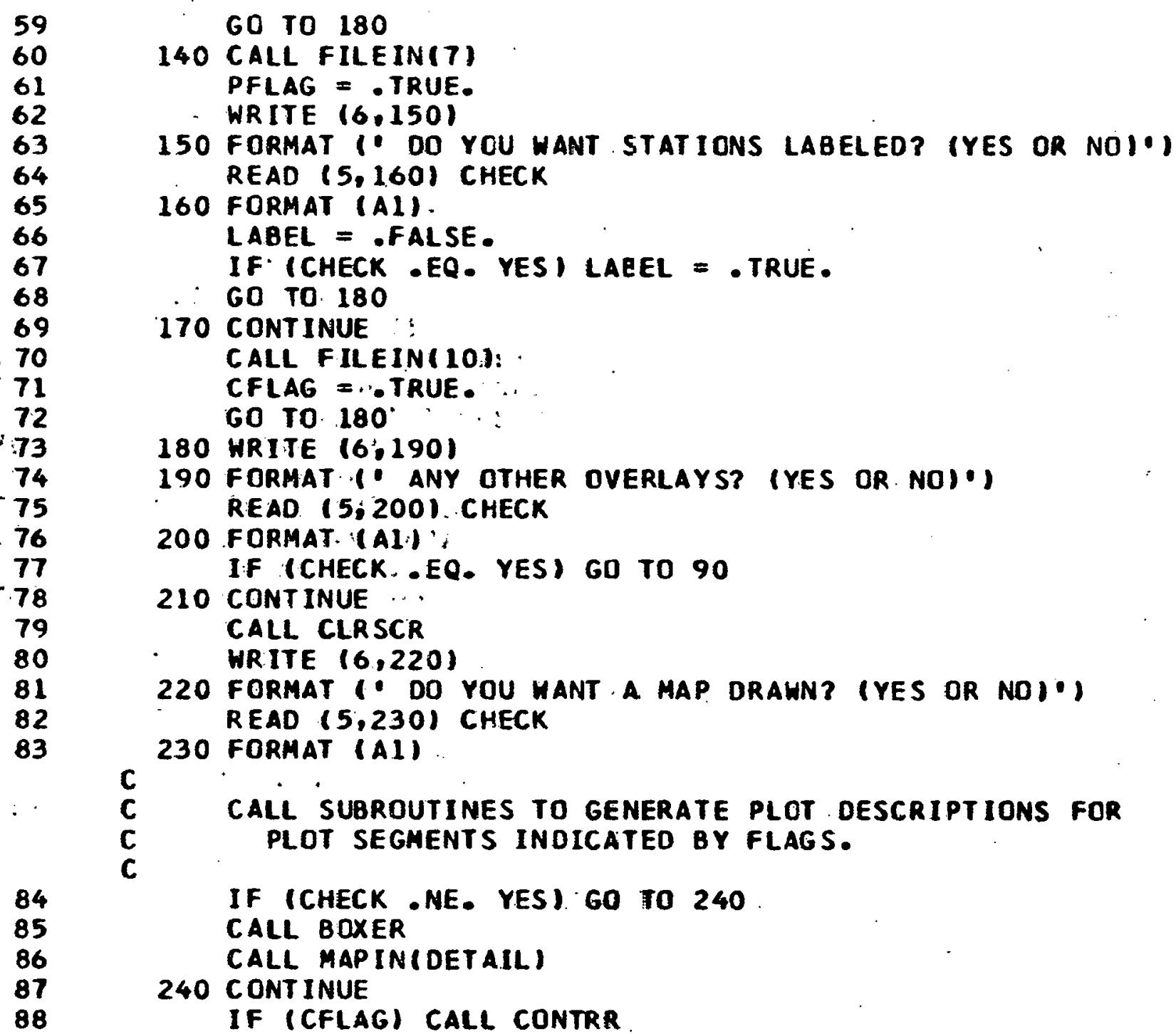




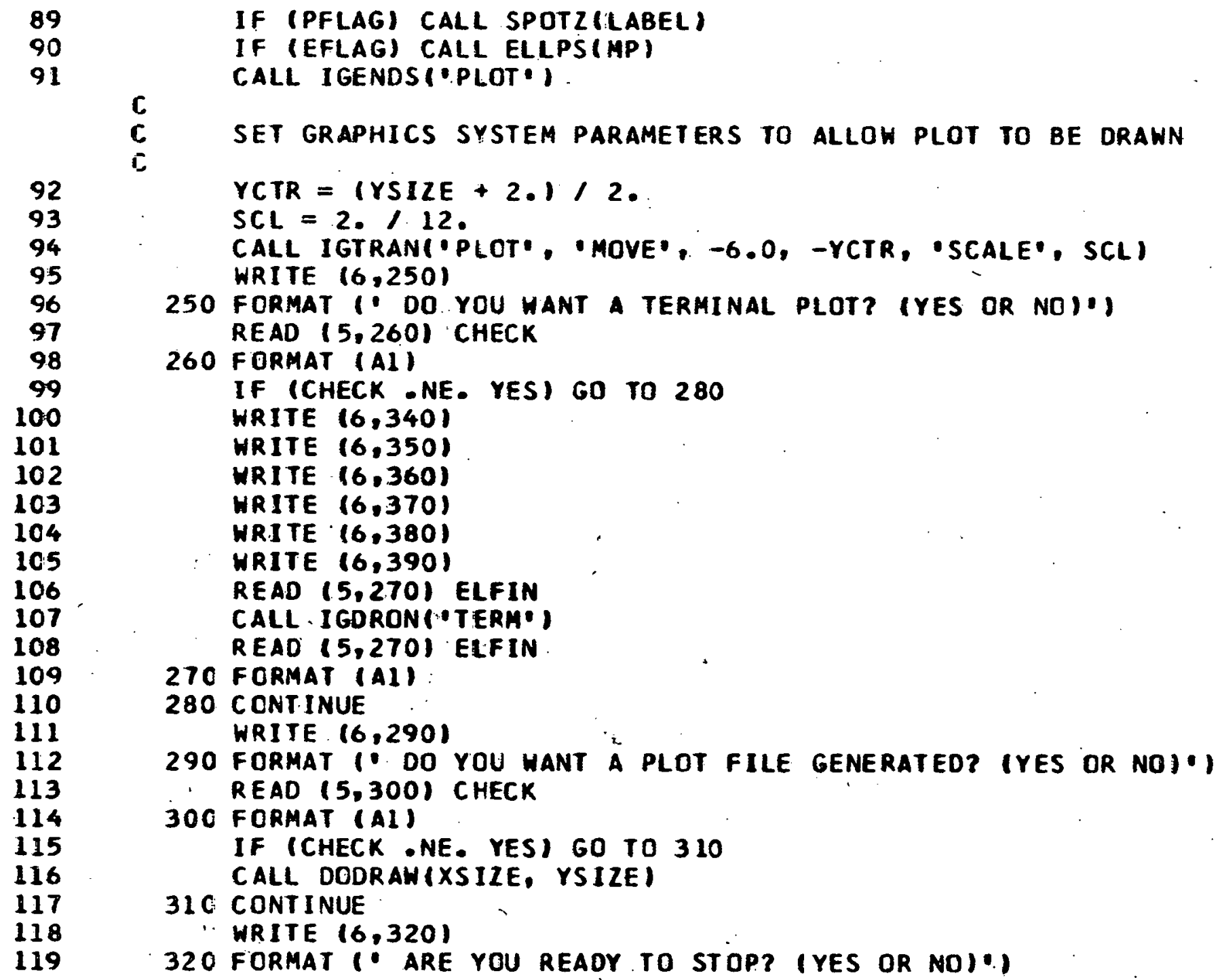

C 


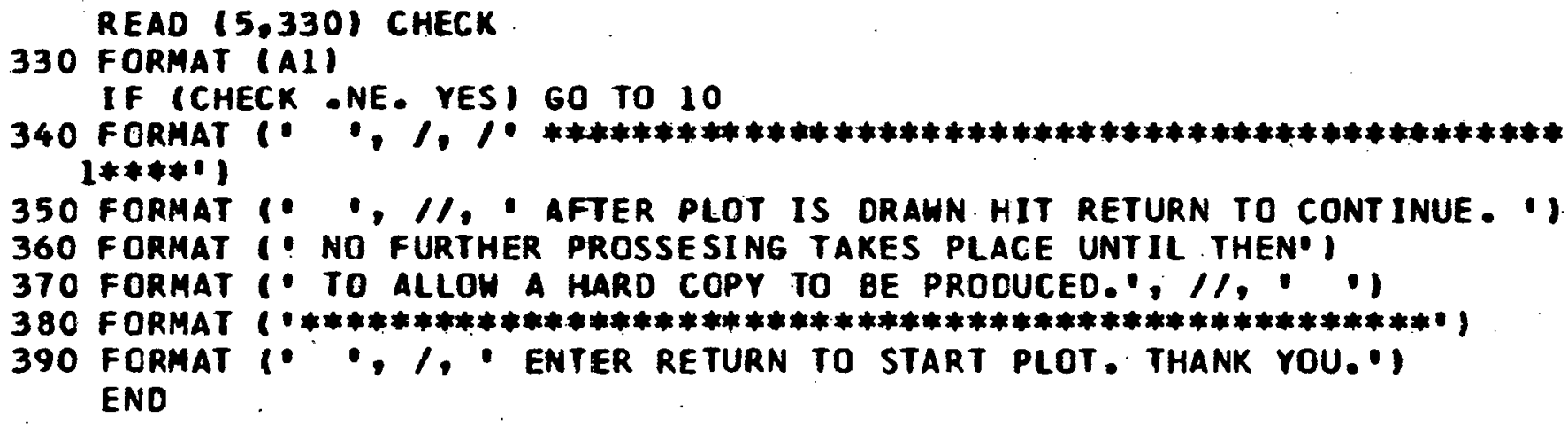


90 FORMAT (F7.4)

IF (XSMAX .GT. 360.0$)$ XSMAX $=360.0$

100 CONT INUE

$S C 3=12.0 /(Y S I Z E+2$.

SIZE2 = YSMAX *12. 11.

CALL IGCTRLI'CALCOMP', 'SIZE', SIZEZ)

CALL IGDRONI'CALCOMPO:

RETURN

END 
FILEIN ASSIGNS DATA FILES TO THE APPROPRIATE LOGICAL DEVICE FOR THE FUNCTION TO BE PERFORMEO. UNIT 4 IS ATTATCHED TO THE FILE CONTAINING CATA FOR ELLIPSE GENERATION, UNIT 7 FOR STATION LOCATION, UNIT 10 FOR CONTOURS, AND UNIT 9 FOR THE. PLOT OUTPUT FILE. UNITS 5 AND 6 ARE USED THROUGHOUT AS THE TERMINAL INPUT AND DUTPUT CHANNELS. AS PER CONVENTION.

\section{LOGICAL*I UNIT(18)}

WR ITE $(6,10)$

10 FORMAT "ENTER NAME OF DATA FILE') READ $(5,20)$ (UNITI 1 ) $1=1,17$ )

20 FORMAT (17AL) GO TO $170,70,70,30,70,70,40,70,50,601, \mathrm{~N}$

30 CALL SETLIOI'4 UNITI GO TO 70

40 CALL SETLIO1'7

$\because$ UNITI

50 CALL SETLIOI'g 60 TO 70

60 CALL SETLIOI'10 GO TO 70

70. CONTINUE RETURN END

๑ UNITI

- UNITI 
SPOTZ READS STATION LOCATION DATA FROM THE FILE ATTACHED TO UNIT 7. THE LOGICAL VARIABLE LABEL ,PASSED TO THE SUBROUTINE, INOICATES WHETHER STATIONS ARE TO BE LABELLED WITH THEIR 3 OR 4 CHARACTER DESIGNATION WHEN PLOTTED.

THE SIZE OF THE SYMBOLS DRANN IS A FUNCTION OF THE SIZE OF THE MAP SPECIFIED IN ORDER TO ALLOW SCALING OF THE MAP. THE SI ZE MAY BE ALTERED BY CHANGING THE NUMERATOR OF. THE FUNCTION DEFINING THE VARIABLE HT

HT $=0.025$, (LONMAX - LONAIN)

READ $(7,10)$ NSTA

c

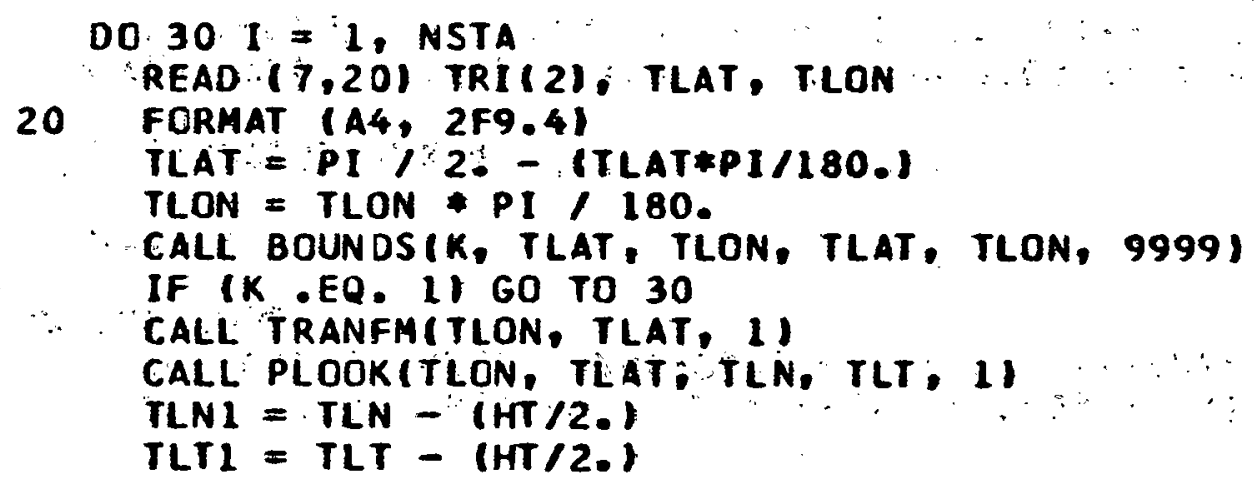


CALL PSYAITLAI, TLTI, HT, TRI(1), 0.0,4,0)

IF (LABEL) CALL PSYMI-0.0, -0.0, HT, TRI(2), $0.0,4,1)$

C 30 CONTINUE

RETURN

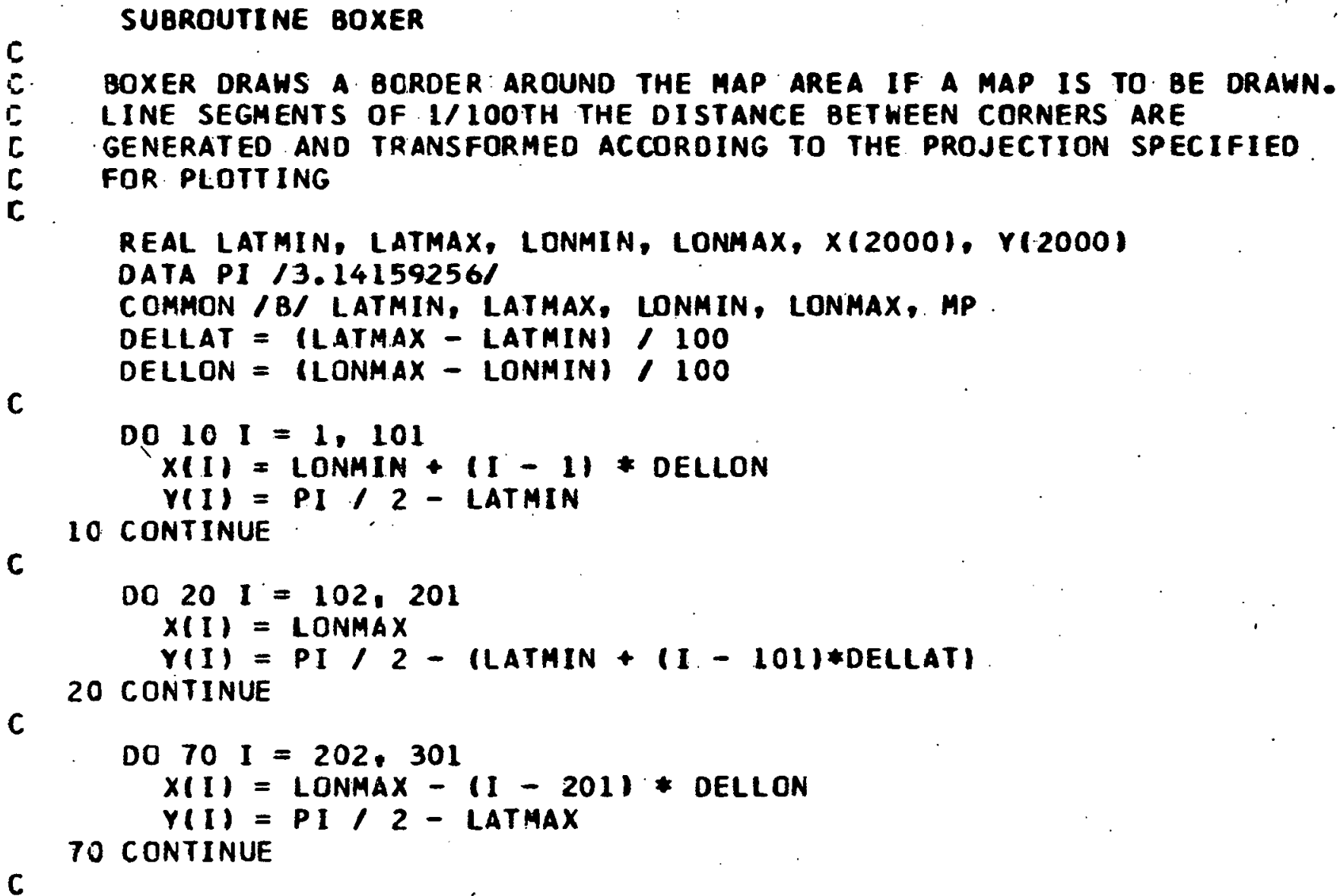




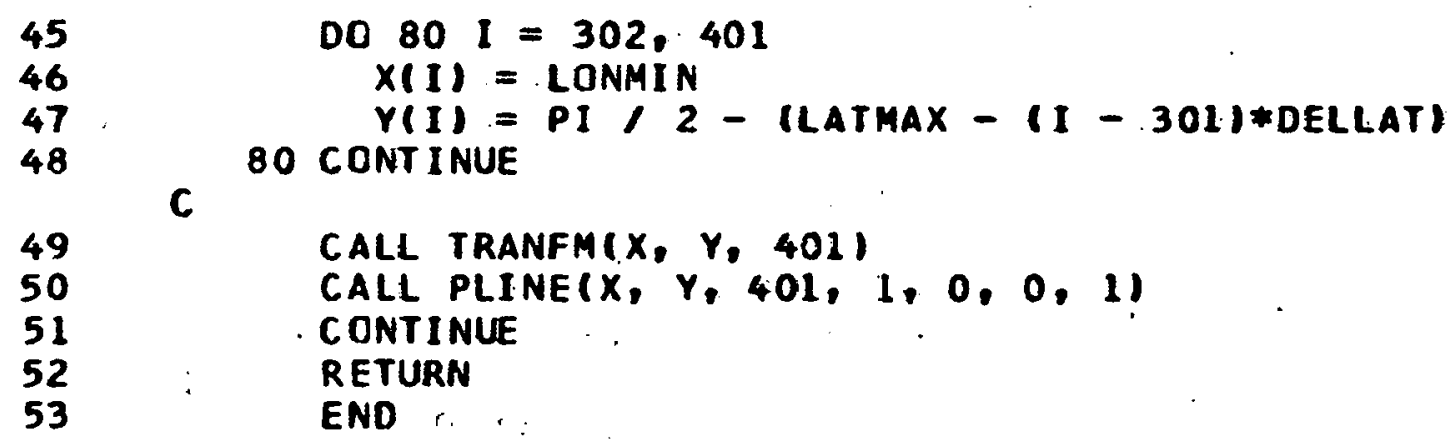


PLTST INITIALIZES THE PLOT TRANSFORMATION PARAMETERS BASED ON THE MAP BOUNDARIES AND PROJECTION SPECIFIED. THE MINIMUM

AND MAXIMUM CO-OROINATES IN THE X AND Y DIRECTION ARE DETERMINED, ALLOWING FOR THE USE OF A RELATIVE INSTEAD OF ABSOLUTE CO-ORDINATE SYSTEM.

\section{COMMON /B/ LATMIN, LATMAX, LONMIN, LONMAX, MP}

REAL LATMIN, LATMAX, LONMIN, LONMAX

DIMENSION A(4), B(4)

DATA PI /3.14159256/

CALL CLRSCR

GO TO $110,10,20,10,10,10,10,10)$, MP

$10 A(1)=$ LONAIN

$B(1)=P I / 2.0-$ LATMIN

$A(2)=$ LONMAX

$B(2)=P I / 2.0$ - LATMAX

CALL TRANFM(A, B, 2$)$

XMIN $=A(I)$

YMIN $=$ B(1)

$X M A X \doteq A(2)$

YMAX $=B(2)$

60. TO 30

C $\cdots$

$20 A(1)=$ (LONMIN + LONMAX) $/ 2$

$B|1|=P I / .2$ - LATMIN

$A(2)=$ LONMIN

$B(2)=P I / 2$ - LATMAX

$A(3)=$ LONMIN

$B(3)=B(1)$

$A(4)=$ LDNMAX

$B(4)=B(1)$

CALL TRANFMIA, B, $4 B$ 
YMIN $=B(1)$

$Y$ MAX $=B(2)$

$X M I N=A(3)$

$X$ MAX $=A(4)$

GO TO 30

30 SCALE $=$ (XMAX $-X M I N) / 10$. SCALE2 = (YMAX - YMIN) /.10. IF (SCALE2 -GT. SCALE) SCALE = SCALE2

YSIZE = IYMAX - YMINI I SCALE

XSIZE = (XMAX - XMIN) SCALE

GO TO 40

40 CONT INUE

RETURN

END 
SUBROUTINE VALINILATMIN, LATMAX, LONMIN, LONMAX, MP, DETAILI

VALIN IS CALLEO FROM SUBROUTINE DEFLT AND ALLOWS THE USER TO DEFINE MAP BOUNDARY, PROJECTION, TYPE (STATE OR COUNTYI, AND DEGREE OF DETAIL PARAMETERS IF ONE OF THE. DEFAULTS IS NOT APPROPRIATE. 
DEFLT INITIALIZES THE MAP GENERATION PARAMETERS - MAXIMUM ANN MINIMUM LATI TUDE ANO LONGITUDE, PROJECTION DESIRED, TYPE ISTATE

C OR COUNTYI. AND DEGREE OF DETAIL. THREE PRE-DEFINED MAPS ARE PROVIDED AS DEFAULTS, OR THE USER MAY SPECIFY. HIE/HER OWN

C PARAMETER SET THROUGH SUBROUTINE VALIN.

\section{INTEGER* 4 CHECK, TMD, THREE, OTHER}

LOGICAL* 1 DETAIL $(3)$, DET $(3,3)$

COMMON /B/ LATMIN, LATMAX, LONMIN, LONMAX, MP

DIMENSION DLTMN(3), DLTMX (3), DLNMN(3), DLNMX (3), LDPROJ 3 )

COMMON LAT(2000), LON(2000), X(2000), Y(2000)

QEAL LAT, LON, LATMIN, LATMAX, LONMIN, LONHAX

DATA THO, THREE, OTHER $1 \cdot 2 \cdot, \cdot 3 \cdot, \cdot 0 \%$

DATA DET $(1,1), \operatorname{DET}(2,1), \operatorname{DET}(3,1): 1,5, \cdot \mathrm{S}^{\circ}, 0^{\circ}$,

DATA DET $(1,2)$, DET $(2,2), \operatorname{DET}(3,2) \% 5 \%, \cdot 2 \cdot, .6 \%$

DATA DET $(1,3), \operatorname{DET}(2,3), \operatorname{DET}(3,3) \% \mathrm{C}, \cdot 2 \cdot, 3 \%$

DATA PI /3.14159256/

WRITE $(6,10)$

10 FORMAT I! BASE MAP DEFAULT PARAMETERS: $\% \%$ ' PARAMETER IAULT 1 DEFAULT 2 DEFAULT $3 \% \%$ MAP TYPE

2 STATE COUNTY? $\%$ DETAIL LEVEL

36 3\%, 6 , MIN LATITUDE

$4 N \quad 41.5 \mathrm{~N}, 1,9$ MAX LATITUDE

$547.5 \mathrm{~N}, \%$ MIN LONGITUDE $66.0 \mathrm{~W}$

$7 \%$ MRQJECTION MERCATOR MERCATOR MERCATOR',

WRITE $(6.20)$

20 FORMAT (: ENTER 1,2 OR 3 FOR DEFAULT LIST OESIRED OR') WRITE $(6,30)$

18

19

30 FORMAT : OTHER to PROVIDE YOUR OWN VAlUES. DefaUlt 1 is ", 1 - the glcbal default.

READ $(5,40)$ CHECK 


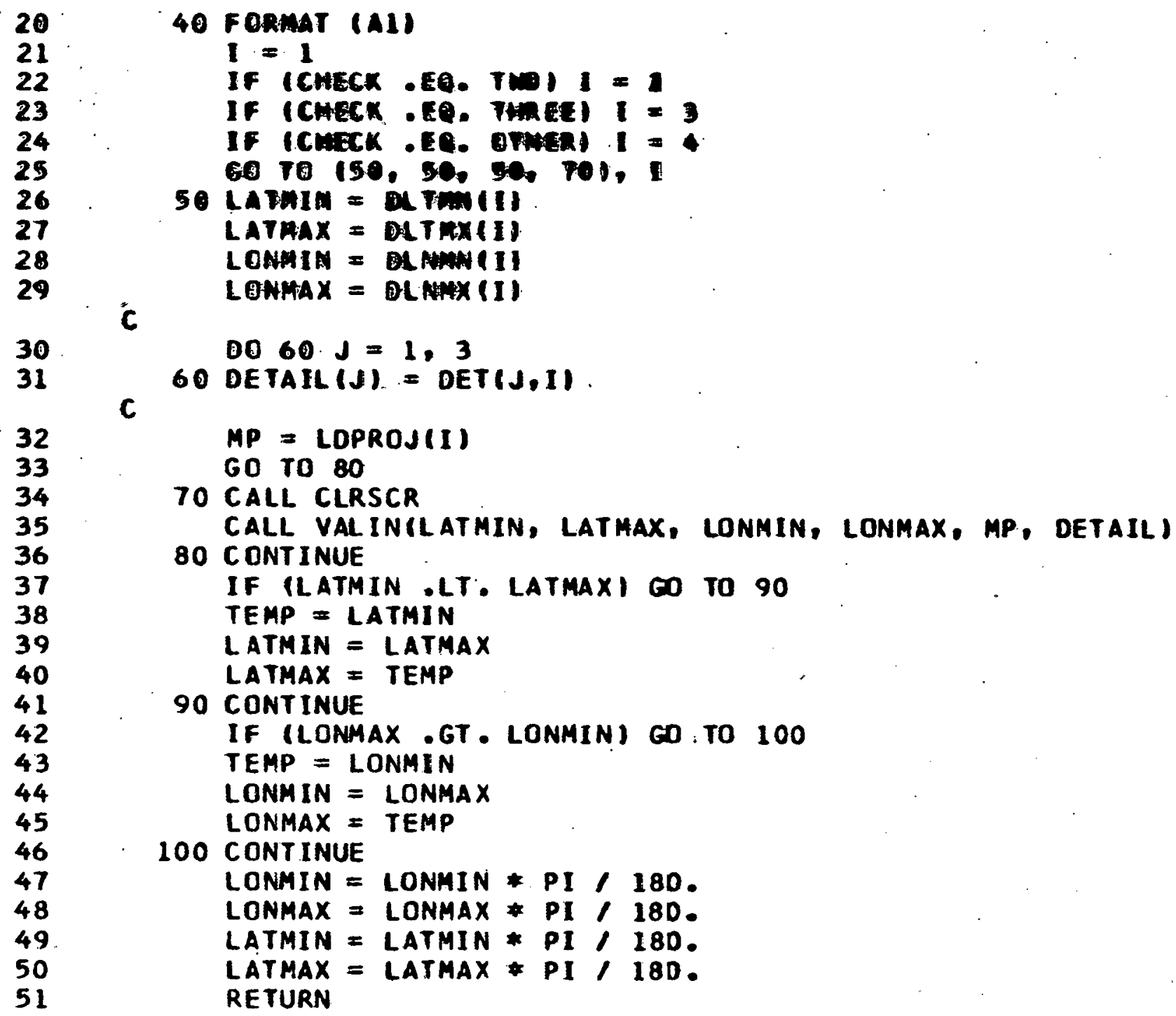

80 CONTINUE

IF (LATMIN IT. LATMAX) EO TO 90

TEMP = LATMIN

LATMIN = LATMAX

LATMAX = TEMP

90 CONTINUE

IF (LONMAX - GT . LONMIN) GD:TO 100

TEMP = LONMIN

LONMIN = LONMAX

LONMAX = TEMP

100 CONT INUE

LONMIN $=$ LONMIN $* P I / 180$.

LONMAX $=$ LONMAX *PI / 180 .

LATMIN = LATMIN *PI/180.

LATMAX = LATMAX * PI / 180.

RETURN 
1

SUBROUTINE CLRSCR
C CLRSCR ERASES THE TERMINAL SCREEN.
C CALL IGBGNS('BLNK')
CALL IGCTRL ' TERMINAL', 'ERASE')
CALL IGENDS('BLNK'
RETURN
END


MAPIN GENERATES THE PLOT DESCRIPTION INFORMATION NEEDED TO DRAW THE SPECIFIED MAP. DATA FOR BORDERS IS STANDARD FIPS FORMAT, AND IS ACCESSED THROUGH THE STATISTICAL RESEARCH LABORATORY 'S STAT:USA/MAP PROGRAM. THO: MIDAS SUBROUTINES FROM THIS PROGRAM, INITXY AND READXY, ARE CALLED TO RETRIEVE THE DATA.

IHIS SUBROUTINE UTILIZES THE LOOP FEATURE OF THE FORTRAN 77 LANGUAGE. HHEN COMPILING, PAR=OVER MUST BE SPECIFIED TO USE THIS OPT ION. MH 


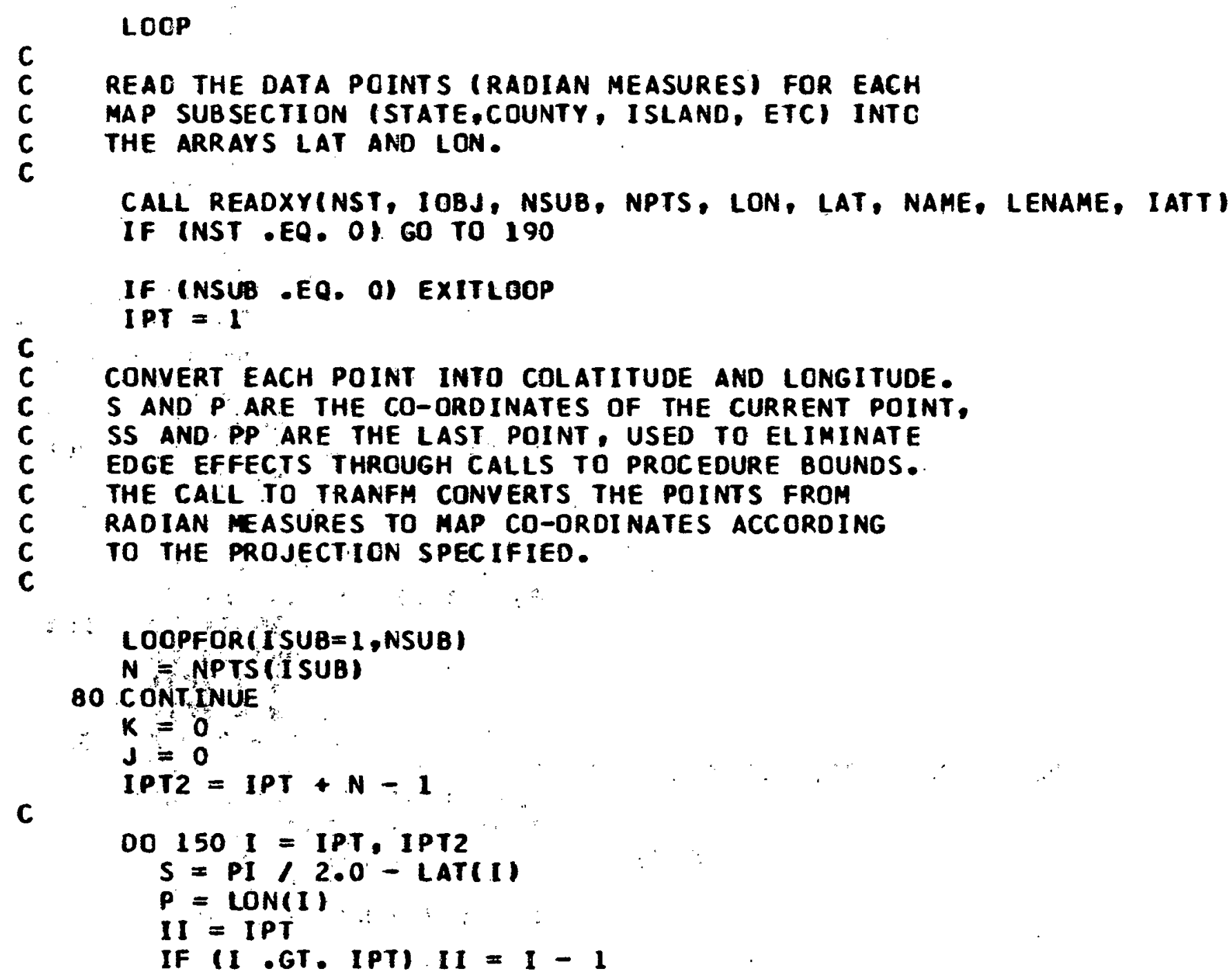




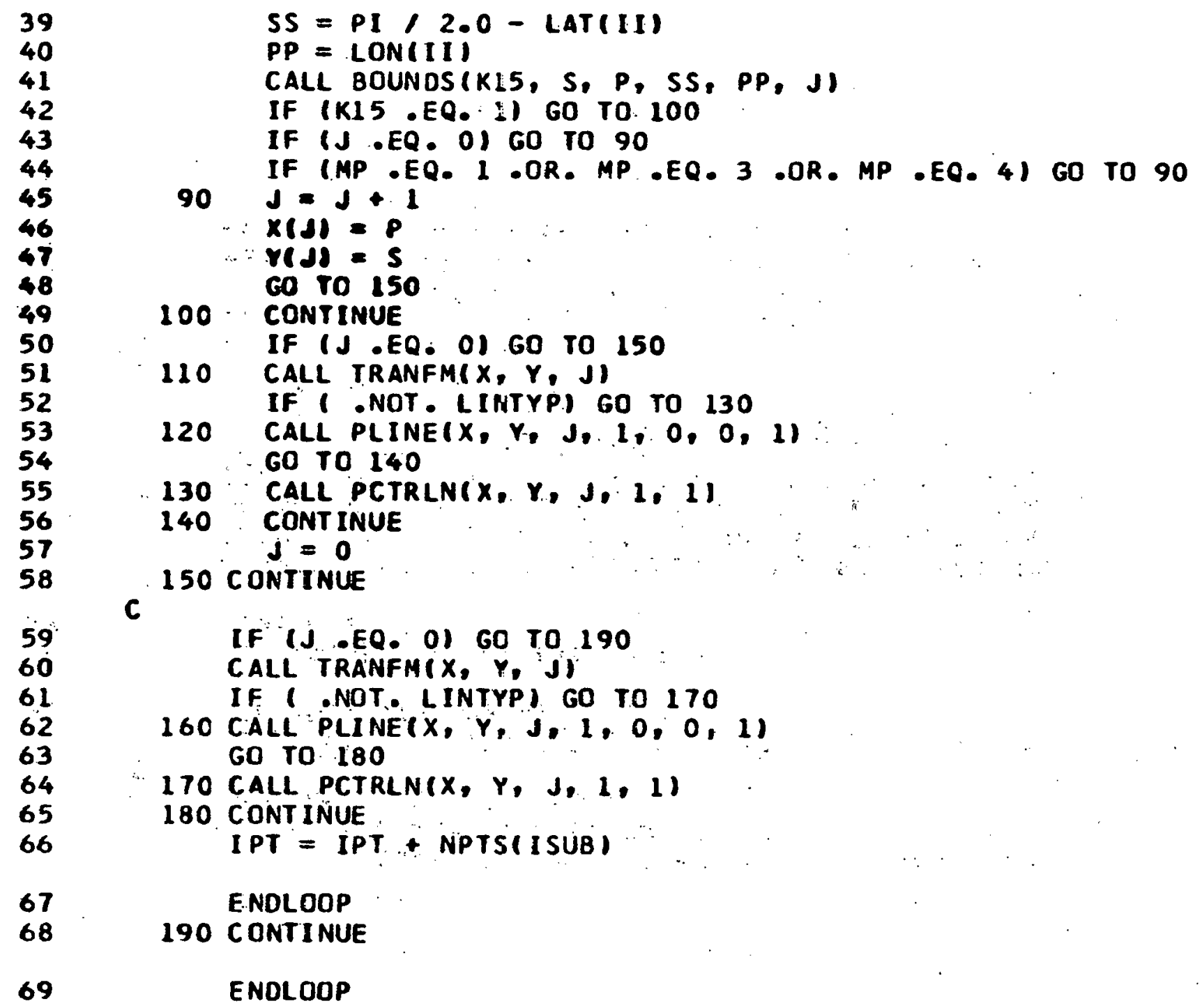


RETURN

END 
SUBROUTINE TAZAR $(X, Y, Z, J)$

REAL $X(J): Y(J), Z(J)$

$C$ ON ENTRY, $X$ CONTAINS E LONGITUDES (RAD)

C $Y$ CONTAINS COLATITUDES. THESE ARE CONVERTED TO

$C X, Y$ COORDINATES OF AN AZIMUTHAL EQUAL AREA PROJECTION,

C WITH THE NORTH POLE BEING THE PROJECTION POLE DO $10 I=1, J$

$C=Y(I)$

$R=2 . * \operatorname{SIN}(C / 2$.

$Y_{1}=-R * \cos (x(1))$

$X I=R * \operatorname{SIN}(X \mid I))$

$X(1)=X 1$

$Y(I)=Y 1$

C

10 CDNT INUE

11

RETUAN

END 


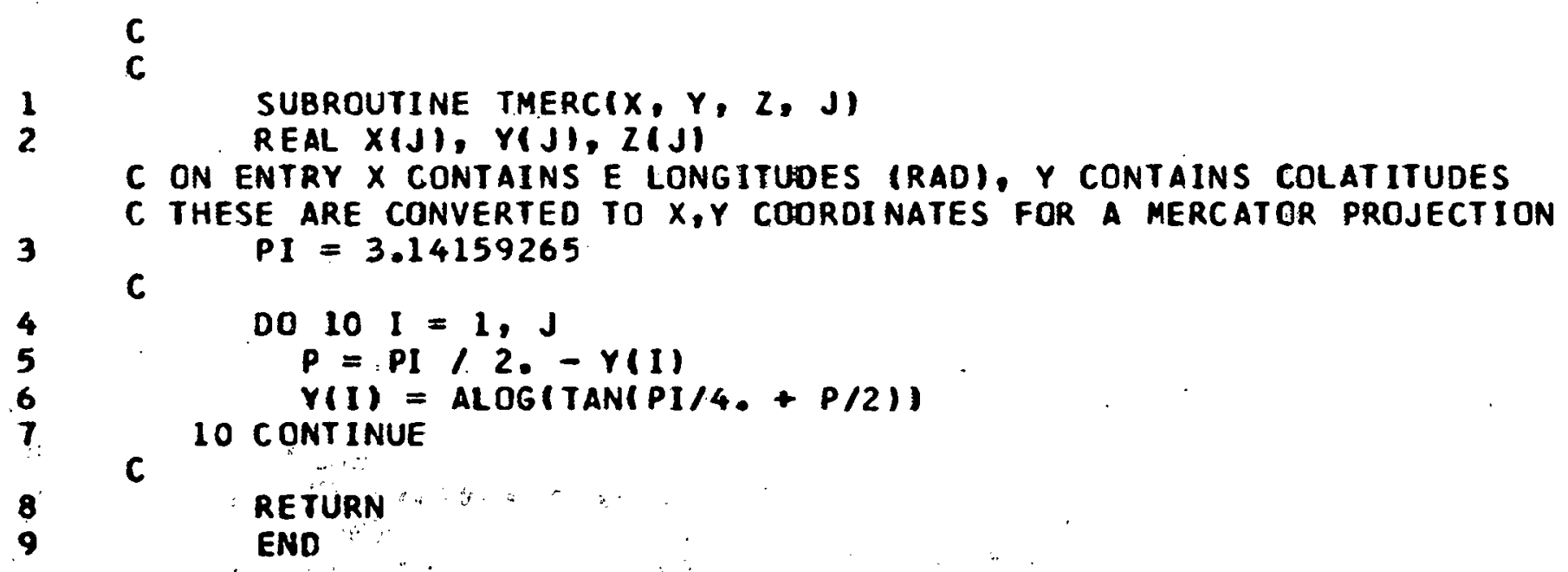




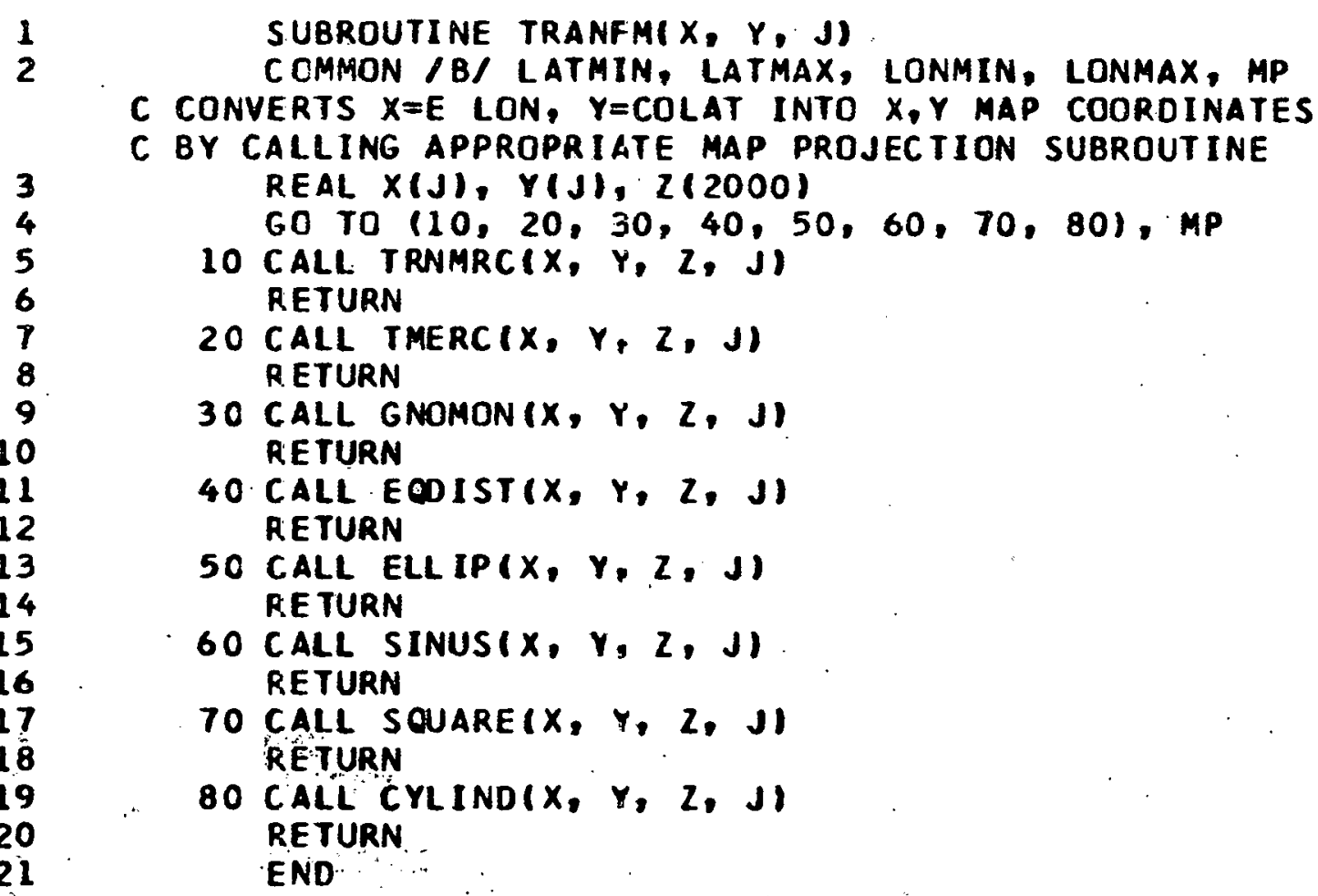


C

1
2
3

SUBROUTINE GNOMONI $X, Y, Z, J)$

COMMON /B/ LATMIN, LATMAX, LONMIN, LONMAX, MP

REAL X(J), Y(J), Z(J), LATMIN, LATMAX, LONAIN, LONMAX

C GNCMONIC PROJECTION - PROJECTION POLE = 9ON, OE

C ON ENTRY, $X=E$ LONG (RAD), $Y=$ COLAT

$C$ THESE ARE CONVERTED TO $X, Y$ MAP COORDINATES

PI $=3.14159256$

PLAT $=$ PI $/ 2.0$

C

PLON = (LONMIN + LONMAX): 2

DO $101=1, J$

$C=P I / 2-Y(I)$

DEN $=(S I N(P L A T) * S I N(C))+(\operatorname{COS}(P L A T) * \operatorname{COS}(C) * \operatorname{COS}(X(I)-P L O N))$

$X X=P L D N+(\operatorname{COS}(C) * S(N) X(I)-P L O N) / D E N)$

$Y Y=(\operatorname{COS}(P L A T) * S I N(C))-(\operatorname{SIN}(P L A T) * \operatorname{COS}(C) * \operatorname{COS}(X(1)-P L O N))$,

1 DEN

$X(1)=X X$

13
14 $\quad$ Y III $=Y Y$

15

$\mathbf{C}$

RETURN

END 
SUBROUTINE EQDISTIX, $Y, Z, J$

REAL $X(J), Y(J), Z(J)$

C AZIMUTHAL EQUIOISTANT PROJECTION ABOUT $N$ POLE

C ON ENTRY, $X=E$ LONG (RAD), $Y=C O L A T$

$C$ THESE ARE CONVERTED TO $X, Y$ MAP COORDINATES

DO $10 I=1, J$

$R=Y(I)$

$T=X(I)$

$X(I)=R * S I N(T)$

$Y I I)=-R * \operatorname{COS}(T)$

10 CONTINUE

9 RETURN

10 . END 


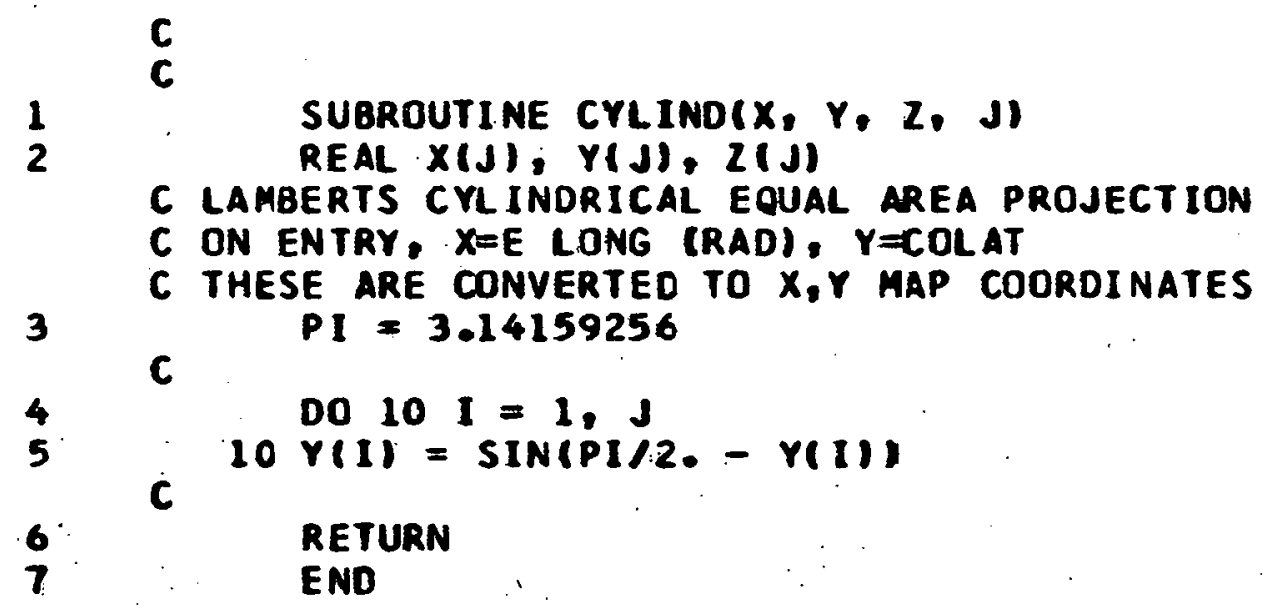




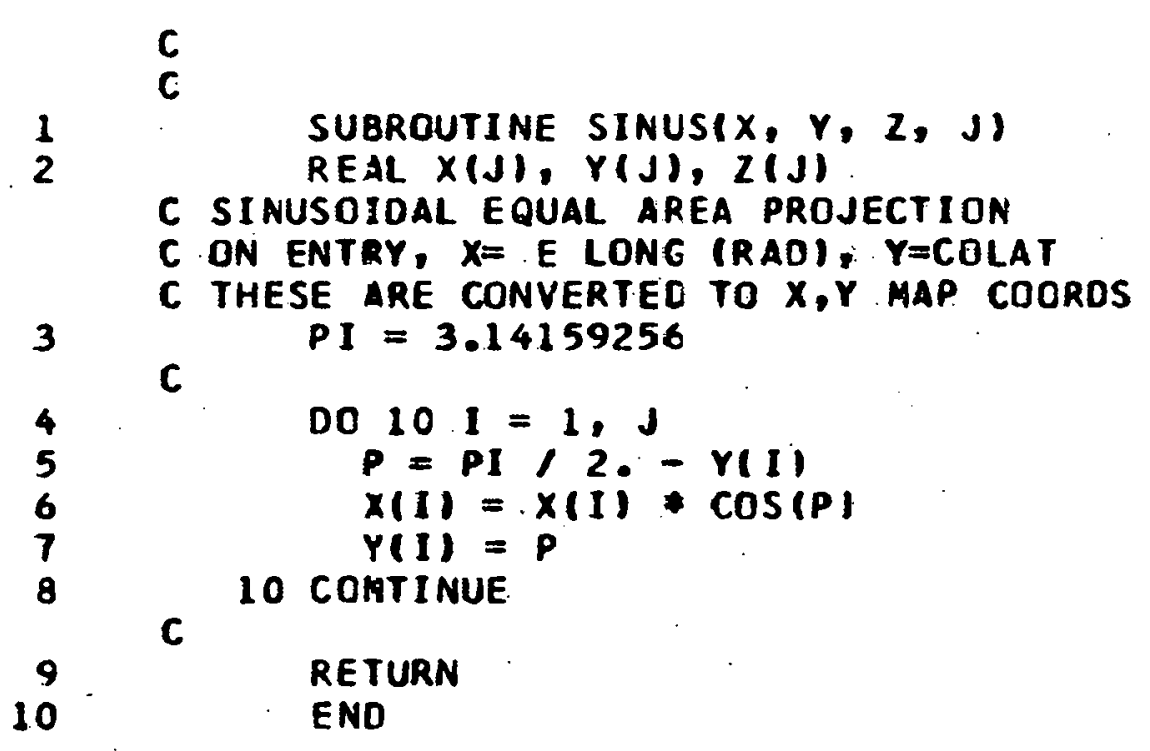




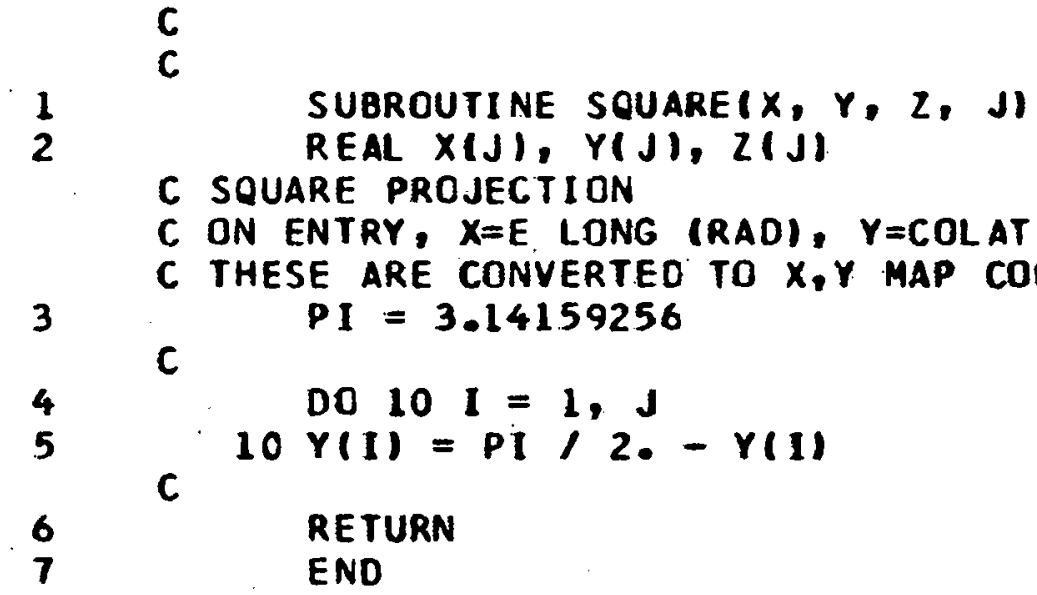




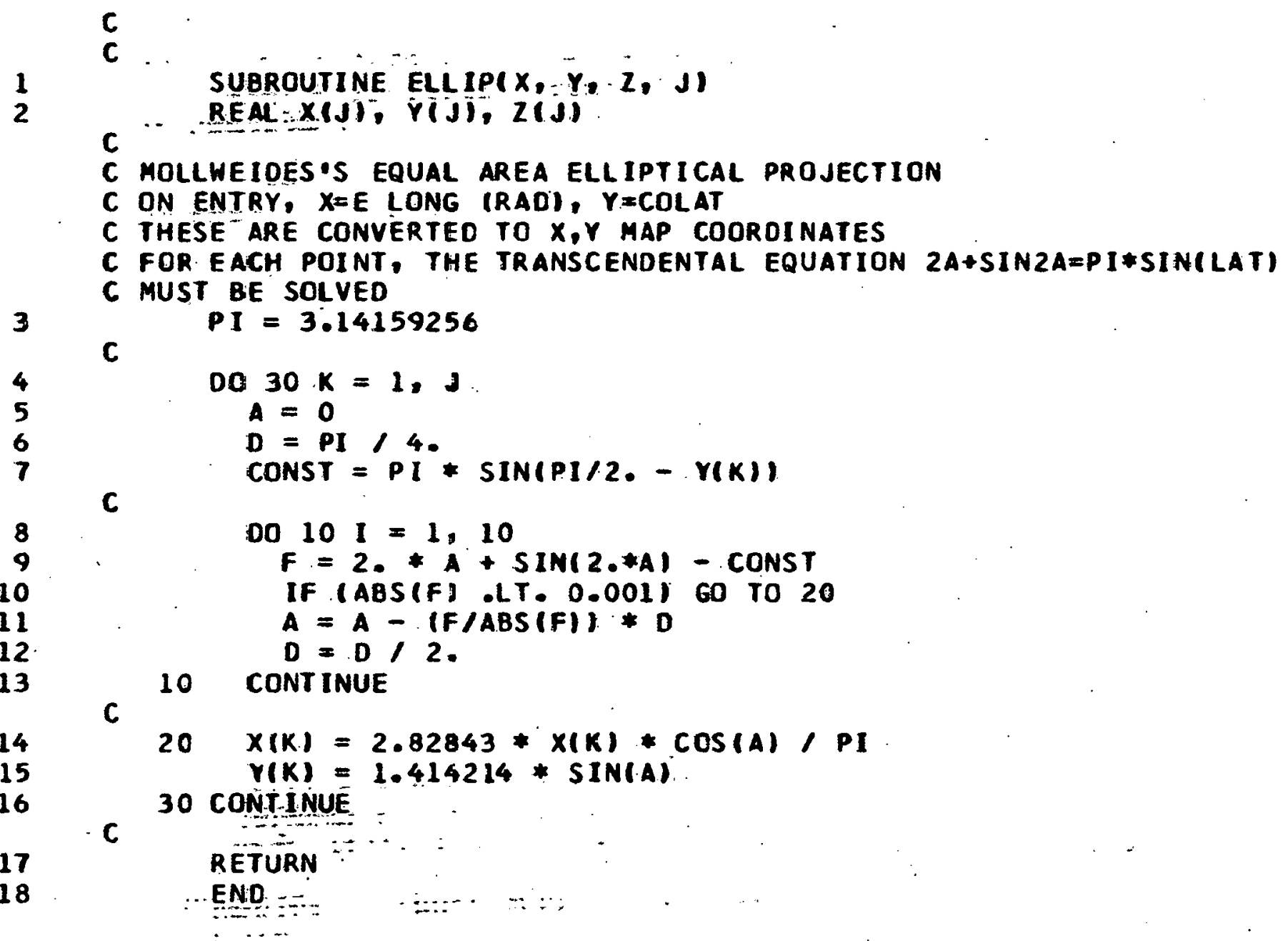


SUBROUTINE BOUNDS (K, COLAT, ELON, LSTY, LSTX, J)

C BOUNDS REMOVES EDGE EFFECTS BY EXAMINING THE CURRENT

C AND LAST POINTS TO BE DRAWN. IF EITHER POINT IS OUTSIDE

C THE MAP BOUNDARY AREA, A POINT ALONG THE SAME SEGMENT

C AS THE ORIGINAL POINTS AND LYING ON THE MAP BOUNDARY

C IS GENERATED. IF BOTH POINTS ARE OUT, BOUNDS CHECKS

C TO SEE IF THE SEGMENT CONNECTING THE POINTS CROSSES

C THROUSH THE MAP REGION, AND, IF SO, GENERATES THE

C APPROPRIATE LINE SEGMENT.

$K=1$ MEANS THE CURRENT POINT IS OUT OF BOUNDS

K2=1 MEANS THE LAST POINT WAS OUT OF BOUNDS

REAL LATMIN, LATMAX, LONMIN, LONMAX, LSTX, LSTY

COMMON LAT (2000), LON(2000), X(2000), Y(2000)

COMMON /B/ LATMIN, LATMAX, LONMIN, LONMAX, MP

$P I=3.14159256$

$K=0$

$K 2=0$

10 CONT INUE

IF (ELON .LT. ILONMIN - 0.0001) .OR. ELON .GT. (LONMAX + 0.0001)

$I K=1$

IF ILSTX .GT. (LONHAX + 0.0001).OR. LSTX .LT. (LONAIN - 0.00011)

10

$1 K 2=1$

XLAT $=1.570796-$ COLAT

Y.LST $=1.570796-$ LSTY

IF (XLAT .LT. ILATMIN - 0.0001) .0R. XLAT .GT. (LATMAX + 0.0001)) $I K=1$

IF (YLST .LT. ILATHIN - 0.0001).OR. YLST .GT. ILATMAX + 0.0001)

$1 K 2=1$

IF (J.EQ. 9999 ) 60 T0 90

If $(K . E Q . K 2)$ GO TO 50

$J=J+1$

If (LSTX . GT. LONMAX . OR. ELON .GT. LONMAX) GO TO 40 
IF (LSTX - LT. LONMIN .OR ELON .LT • LONMIN) GO TO 30

IF (YLST . GT. LATMAX OOR. XLAT .GT . LATMAX) GO TO 20

$Y(J)=P I / 2$ - (LATMIN)

$X(J)=E L O N+($ ILATMIN - XLAT) $\#(E L O N-L S T X) /(X L A T-Y L S T))$ GO 1090

$20 Y(J)=P I / 2$ - (LATMAX)

$X(J)=E L O N+($ ILATMAX - XLAT I*IELON - LSTX)/(XLAT. - YLST) $)$

60. TO 90

$30 X(J)=$ LONMIN

$Y(J)=P I / 2-(X L A T+1(L O N M I N-E L O N) *(X L A T-Y L S T I / I E L O N-$ ILSTXIII

GO 10.90

$40 \times(J)=$ LONMAX -0.00001

$Y(J)=P I / 2$ - (XLAT + (ILONMAX - ELON)*(XLAT - YLSTI/IELON $11 S(X) \|$

GD TO 90

50 CONTINUE

IF (K.EQ. O) GO TO 90

IF (ELON IGT. LONMAX .AND. LSTX . GT. LONMAX) GO TO 90

IF (ELON - LT. LONMIN . AND. LSTX - LT. LONMIN) GO TO 90

IF (XLAT . GT. LATMAX.AND. YLST, GT. LATMAX) .60 TO 90

IF (XLAT -LT. LATMIN A AND. YLST .LT. LATMIN) GO TO 90

IF (ELON . NE. LSTX) GO TO 60

$X(1)=E L O N$

$x(2)=x(1)$

$Y(1)=P I / 2-$ LATMIN

$Y(2)=P I / 2$ - LATMAX

$J J=2$

60 TO 80

60 CONTINUE

IF IXLAT .NE. YLST I GO TO 70

$Y(1)=P I / 2-X L A T$

$Y(2)=Y(1)$

$X(1)=$ LONMIN 


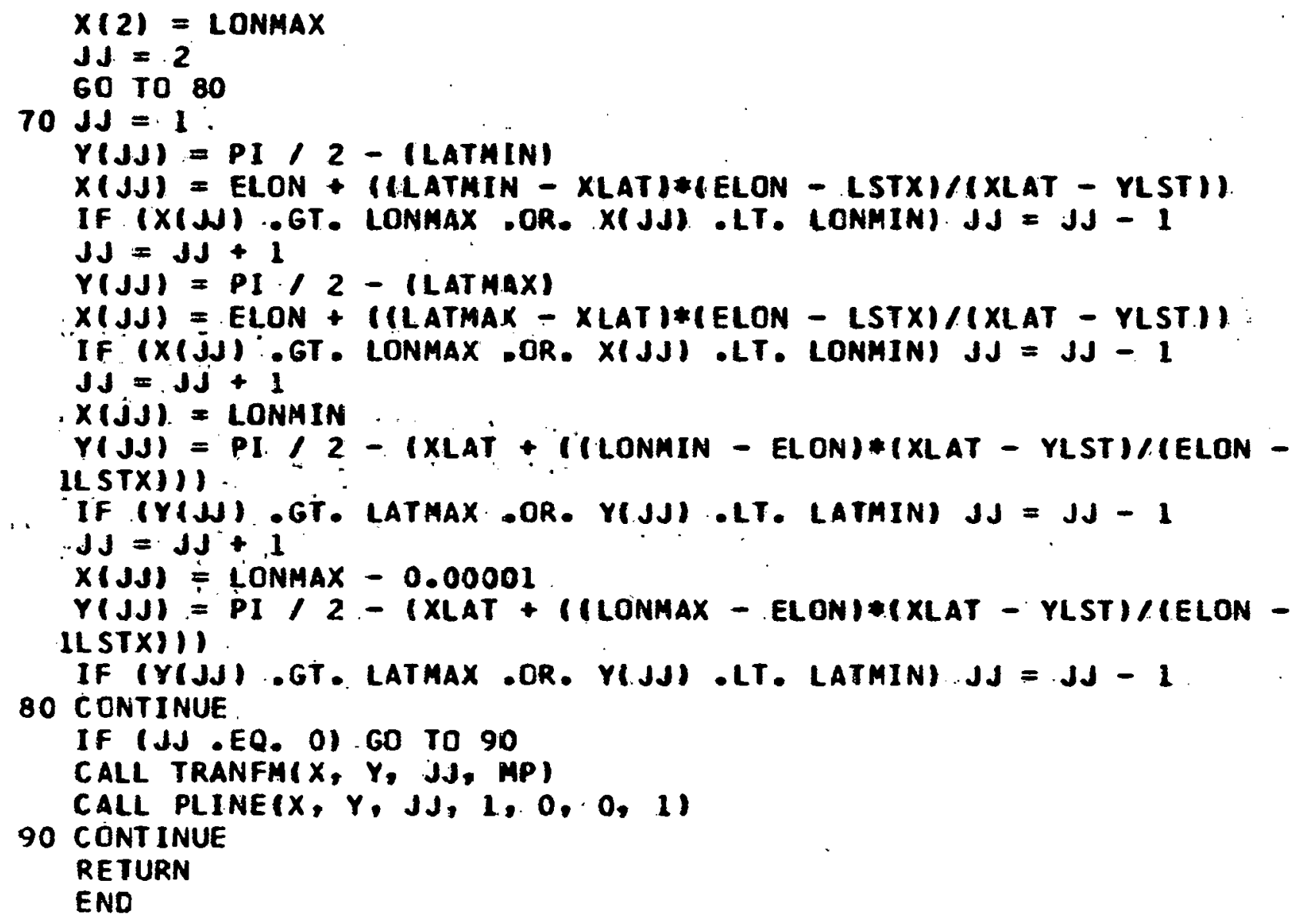


C LATLIN GENERATES A LATITUDE/LONGITUDE GRID. THE LOGICAL VARIABLE DASH CETERMINES HHETHER SOLIO OR DASHED LINES ARE DRAWN. 
$X(I)=$ LONMIN + DELCON $*(1-1)$

C

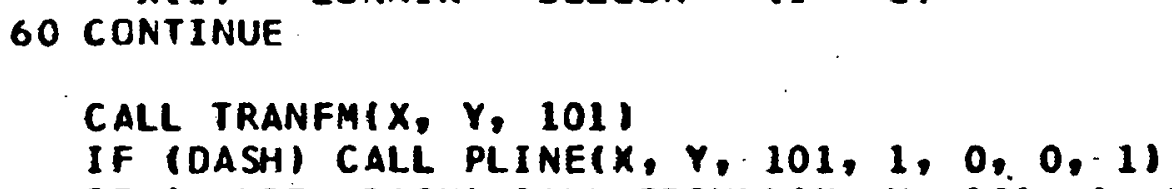




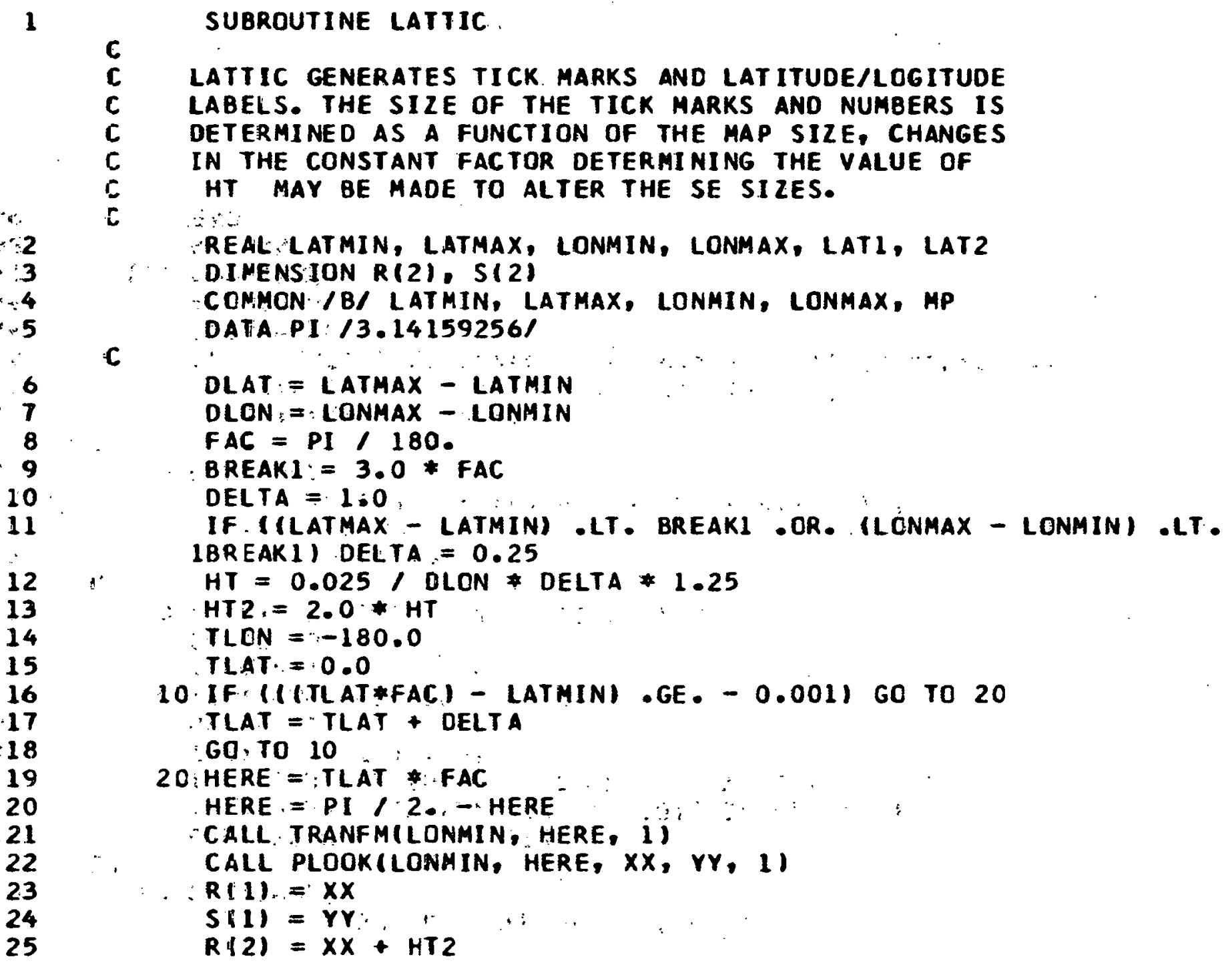


CALL PLINE(R, S, 2, $1,0,0,0)$

$Q=X X-H T 2$

$Y 2=Y Y+H T 2$

CALL PNUMBR 10, Y2, HT, TLAT, 270.0, JF4.2*\%, 01

HERE = PI / 2. - TLAT * FAC

CALL TRANFM(LONMAX, HERE, 1$)$

CALL PLOOK ILONMAX, HERE, $X X, Y Y, I 1$

$R(1)=X X$

$R(2)=X X-H T 2$

$S(1)=Y Y$

$S(2)=Y Y$

CALL PLINE(R, S, 2, 1, 0, 0, 01

TLAT = TLAT + DELTA

IF (ITLAT*FAC - LATMAX).GT. 0.001) GO TO 30 60 TO 20

30 CONTINUE.

40 IF (ITLON*FAC - LONHIN) .GE. - 0.001) GO TO 50 TLON = TLON + DELTA 60 TO 40

50 HERE = TLON * FAC

LAT2 = PI / 2. - LATMIN

CALL TRANFM(HERE, LAT2, 13

CALL PLOOKIHERE, LAT2, $X X, Y, 11$

$R(1)=X X$

$S(1)=Y Y$

$R(2)=X X$

$S(2)=Y Y+H T 2$

CALL PLINE(R, S, $2,1,0,0,0)$

$Q=X X-3 \cdot * H T$

$Y 2=Y Y-3 . H T$

IF (HT.GT. 0.3 ) Y2 $=$ YY -0.9

C ALL PNUMBR 1Q, Y2, HT, TLON, 0.0, JF4.2*०,0)

HERE = TLON * FAC 
LAT I = PI / 2. - LATMAX

CALL TRANFMIHERE, LAT 1,11

CALL PLOOK IHERE, LATI, XX, YY, II

$R(1)=X x$

$R(2)=X X$

$S(1)=Y Y$

$S(2)=Y Y-H T 2$

CALL PLINE(R, S, 2, 1, 0, 0, 01

TLON = TLON + DELTA

IF (ILON*FAC - LONMAX).GT.0.001) G0 TO 60 60 TO 50

60 CONT INUE

RETURN

END 
C ELLPS DRAWS ELLIPSES CENTERED AT LATILLN WITH

C MAJOR AXIS A ANO MINOR AXIS B, IMCLINED AT

C AZMTH DEGREES CLOCKWISE FROM THE HORIZONTAL.

C SUBROUTINE PELIPS FROM THE *PLOTSYS SYSTEM

C GENERATES THE ACTUAL CO-ORDINATE POINTS,

C. THE Call to pline places a POINT IN THE Ellipse

C CENTER. ELLIPSES WITH MAJOR AXIS LARGER THAN THE

C DISTANCE BETHEEN DATA POINTS ARE NOT DRAWN TO

C PREVENT OVERLAPPING; ONLY THE CENTER POINTS ARE DRAWN.

$P I=3.14159256$

REAL LAT, LON, MAG, LAT1, LON1, MAGI

$N=1$

10 CONTINUE

IF (N.EQ. 2) 60 TO 30

READ $(4,20, E N D=60)$ LAT, LON, MAG, A, B, AZMTH

IF (N ANE. 1 ) GO TO 40

READ $(4,20)$ LAT1, LON1, MAG, AA, BB, AZ1

SZMAX = ABS (LAT - LATI) * 111.195

SZMAX2 = ABS (LON - LON1) * 111.195

IF (SZMAXZ -GT . SZMAX) SZMAX = SZMAXZ

20 FORMAT (1X, 2F $7,2, \cdots F 4.2, \therefore$ F $12.2, F 5.1$ ) 60 TO: 40

$30 \angle A T^{\circ}=\angle A T 1$

LON $=$ LONL

MAG MAGL

$A:=A A$

$\dot{B}=\mathbf{B B}$

$A Z M T H=A Z 1$

40 CONT INUE

$Y=P I / 2-(L A T * P I / 180$.

$X=-(L O N * P I / 180$. 
$A 1=A / 111.195 *(P I / 180$.

$B 1=B / 111.195 *(P 1 / 180$.

ANGL $=90 .-A Z M T H$

CALL BOUNDSIK, $Y, X, Y, X, 9999)$

IF (K .EQ. 1 ) 60 TO 50

CALL TRANFM(X, $Y, 1$, MP)

I.F (AL -LE . O) :G0 TO 50

HT $=0.001$

CALL: PLNSYMIHT I

CALL PLINE $(X, Y, 1,1,1,11,1)$

IF (A.GT. SZMAX) GO TO 50

CALL PELIPS(X, Y, AL, B1, ANGL, $0,360,11)$

$50 N=N+1$

GO TO 10

60 CONTINUE

RETURN

END 
C CONTRR SETS UP THE PARAMETER LIST FOR THE CONTOURING

C SUBROUTINES. THESE PARAMETERS ARE USED IN DETERMINING

C ARRAY SUBSCRIPJS AND ARRAY ELEMENT VALUES IN SETARY.

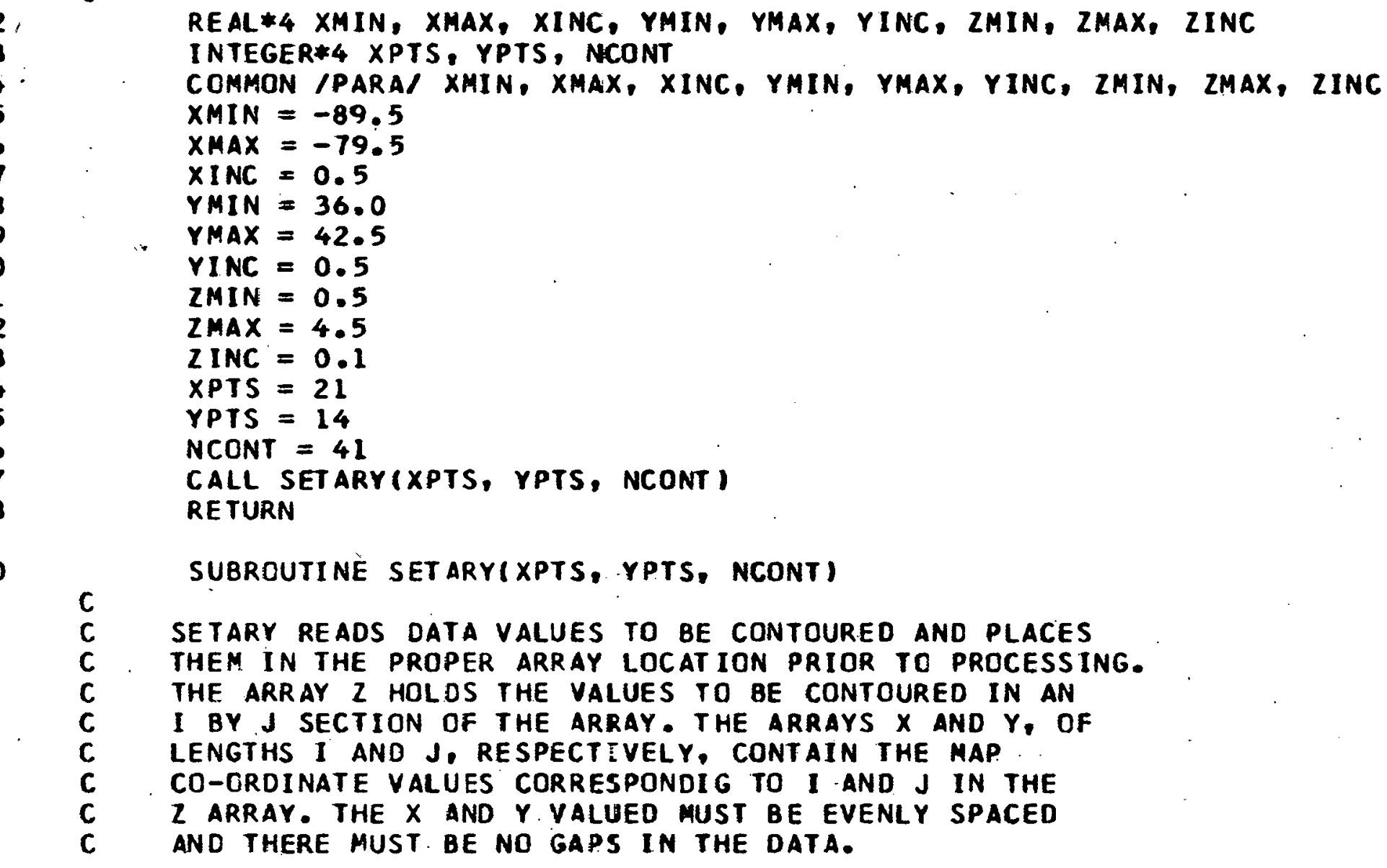


$c$ 
CONTUR DETERMINES WHERE CONTOUR SEGMENTS ARE NEEDED. CELLS ARE DEF INED BY CO-ORDINATES $(I, J),(I, J+1)$, $(1+1, j)$, and $(1+1, j+1)$. THE 2 aRRAY VALUES at THESE POINTS ARE EXAMINED ANO THE MAXIMUM AND MINIMUM VALUES FOR THE CELL ARE DETERMINED. THE CZ ARRAY HOLDS AN ORDERED LIST OF THE VALUES FOR WHICH CONTOUR LINES ARE TO BE DRAWN: IF ANY OF THE ELEMENTS OF THE C $Z$ ARRAY LIE BETHEEN THE MINIMUM AND MAXIMUM CELl Values, a CaLl TO CTQQ IS MADE TO Generate THE CONTOUR LINE SEGMENTS IN THAT CELL.

REAL*4 LATMIN, LATMAX, LONMIN, LONMAX COMMON /B/ LATMIN, LATMAX, LONMIN, LONMAX, MP COMMON /VEC/ $X(100), Y(100), Z(100,100), C Z(100)$ COMMON /PARA/ XMIN, XMAX, XINC, YMIN, YMAX, YINC, ZMIN, ZMAX, ZINC COMMON /CONCOM/ LOHER, UPPER, XL, XR, XC, YL, YU, YC, ZLL, ZUL. ZLR, ZUR

INTEGER*4 LOWER, UPPER, CONCNT, CCPI, IXPT, IYPT

DATA PI /3.14159256/

CZMAX $=$ CZ(NC)

$C Z M I N=C Z(1)$

IYMI $=I Y P T-1$

C

$Y U=Y(1)$

DO. $50 J=1$, IYMI

$\mathbf{V L}=\mathbf{Y U}$

$Y U=Y(J+1)$

$Y C=0.5 *(Y L+Y U)$

$X R=X(1)$

$Z L R=Z(1, J)$

C

$Z U R=2(1, J+1)$ 


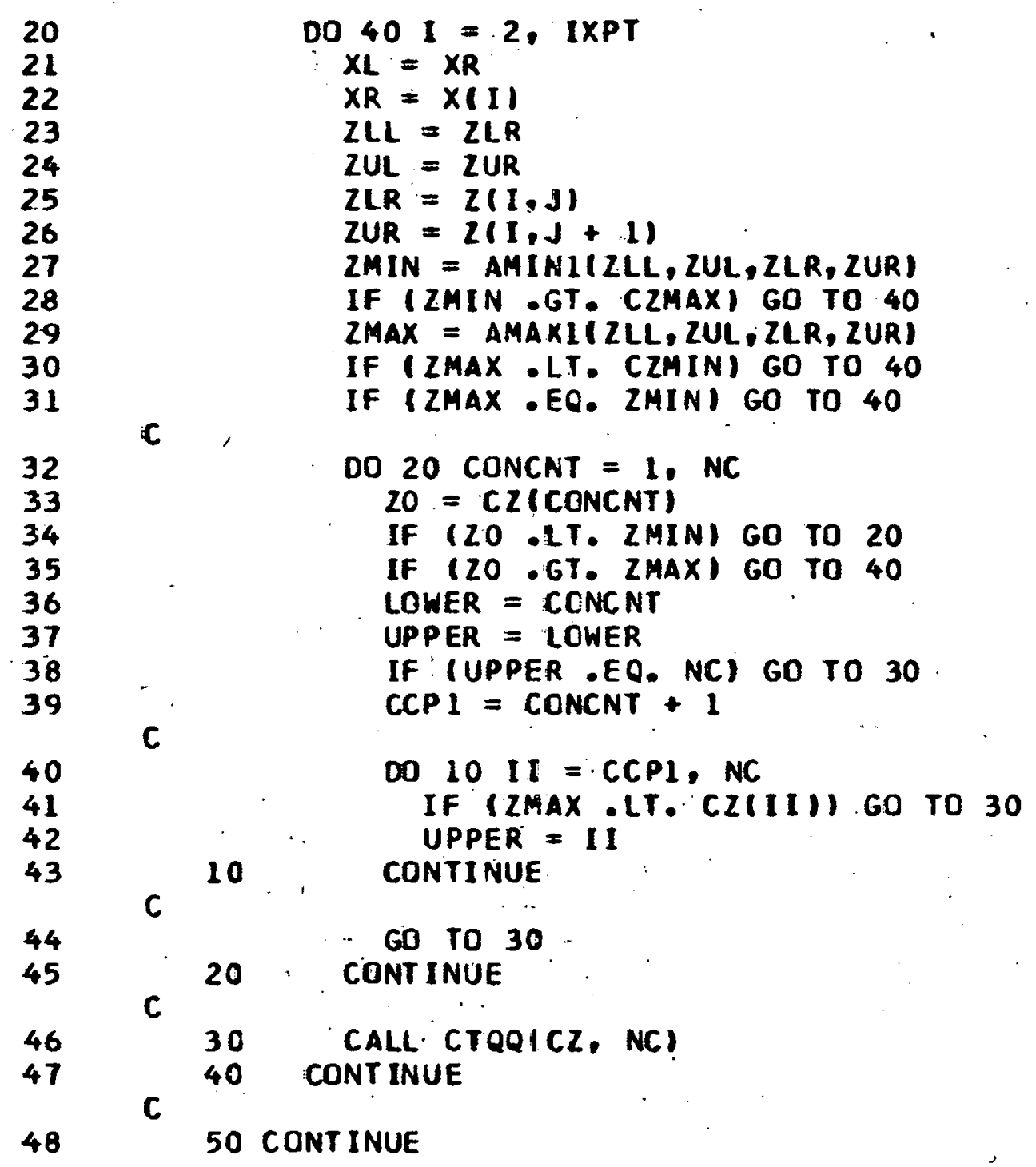




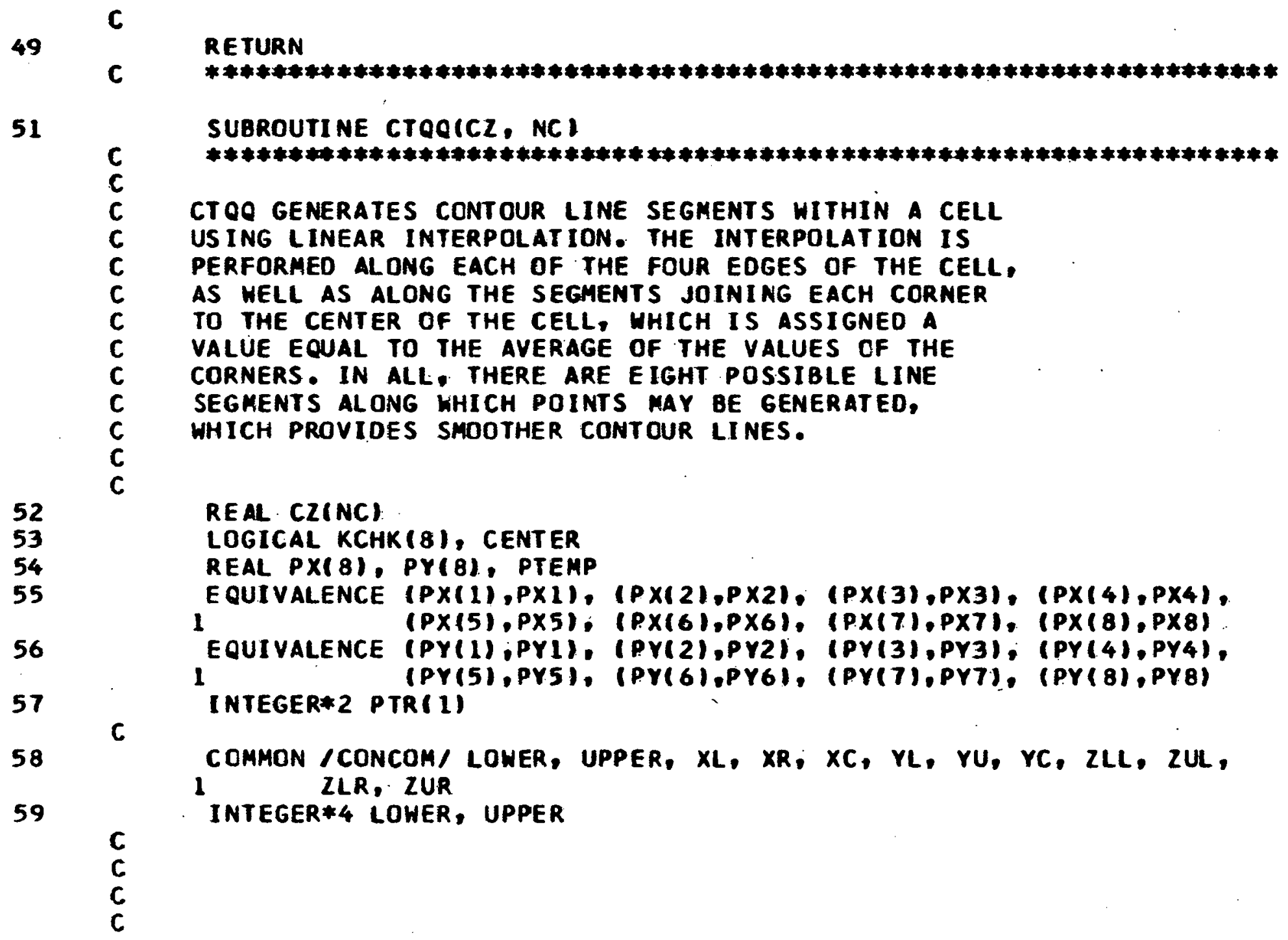

\section{REAL CZ(NC)}

LOGICAL KCHK(8), CENTER

REAL PX( 8), PY (8), PTEMP

EQUIVALENCE (PX(1),PX1), (PX(2),PX2), (PX(3),PX3), (PX(4),PX4), 1 (PX(5),PX5); (PX(6),PX6), (PX(7),PX7), (PX(8),PX8).

EQUIVALENCE (PY (1),PY1), (PY (2),PY2), (PY (3),PY3), (PY (4),PY4), 1 (PY (5),PY5), (PY 6),PY6), (PY (7), PY7), (PY (8),PY8)

59 INTEGER*2 PTR(1) COMMON /CONCOM/ LOWER, UPPER, XL, XR; XC, YL, YU, YC, ZLL, ZUL, 1 ZLR, ZUR

$c$
$c$
$c$
$c$ 


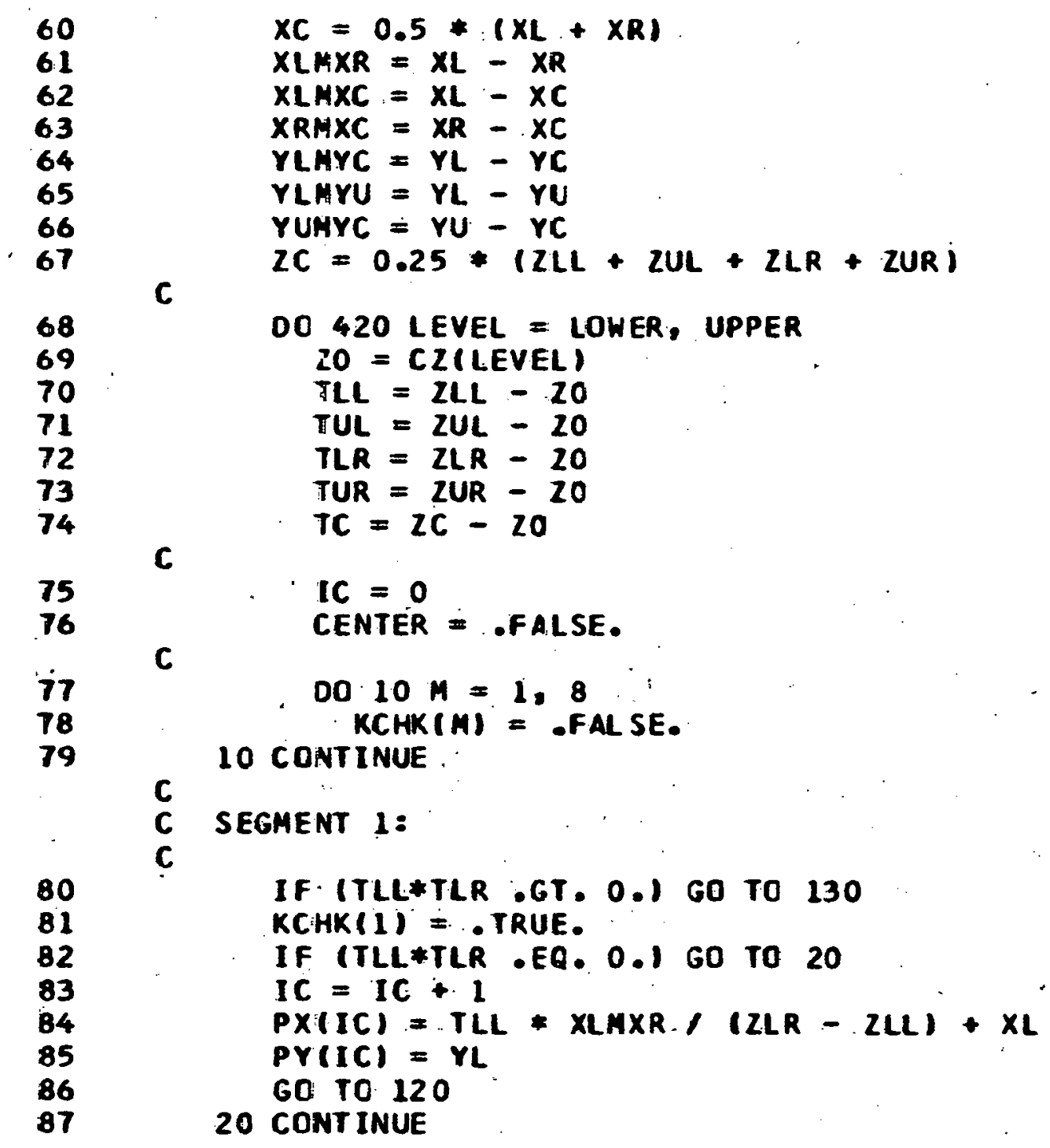




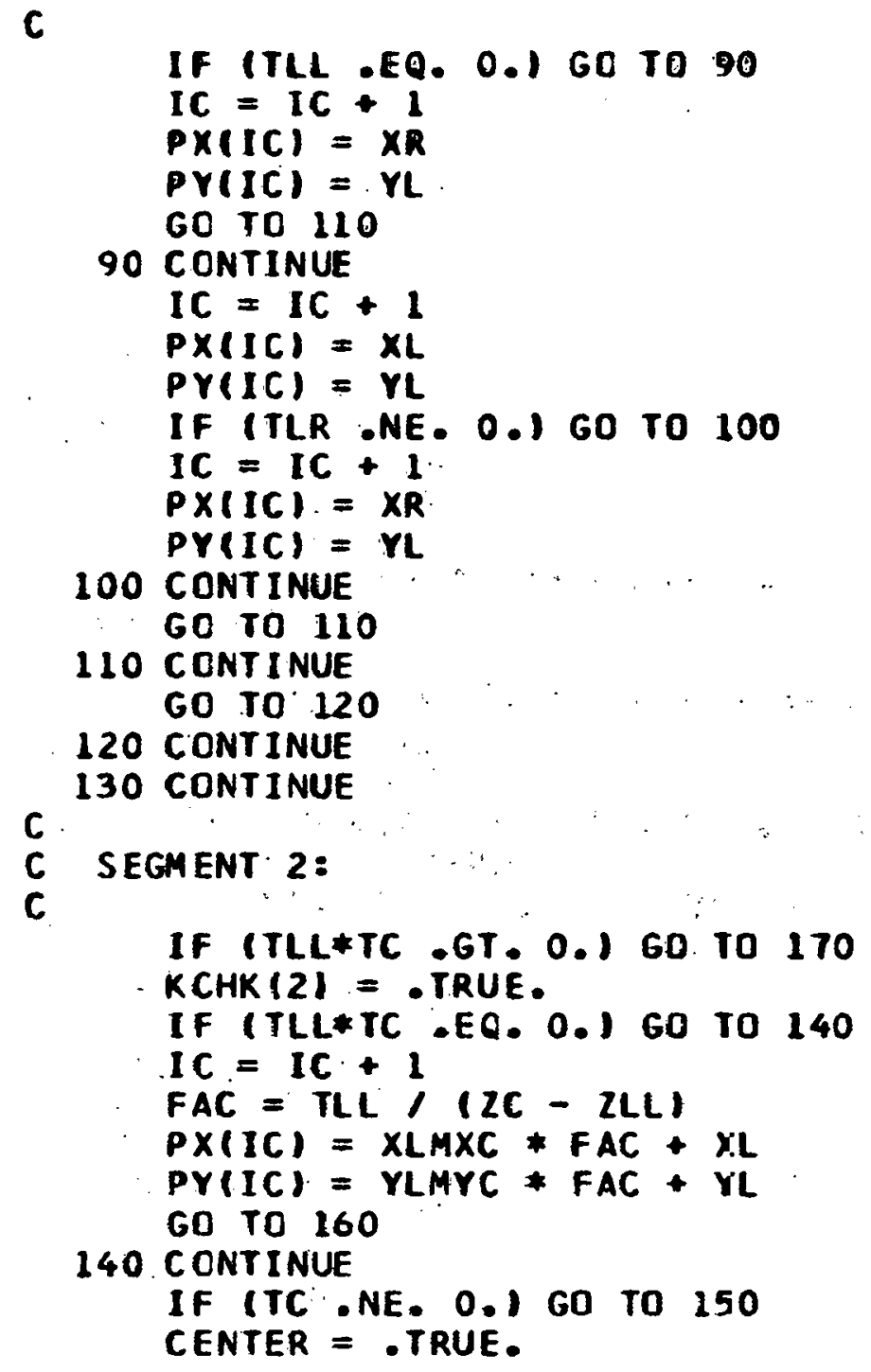




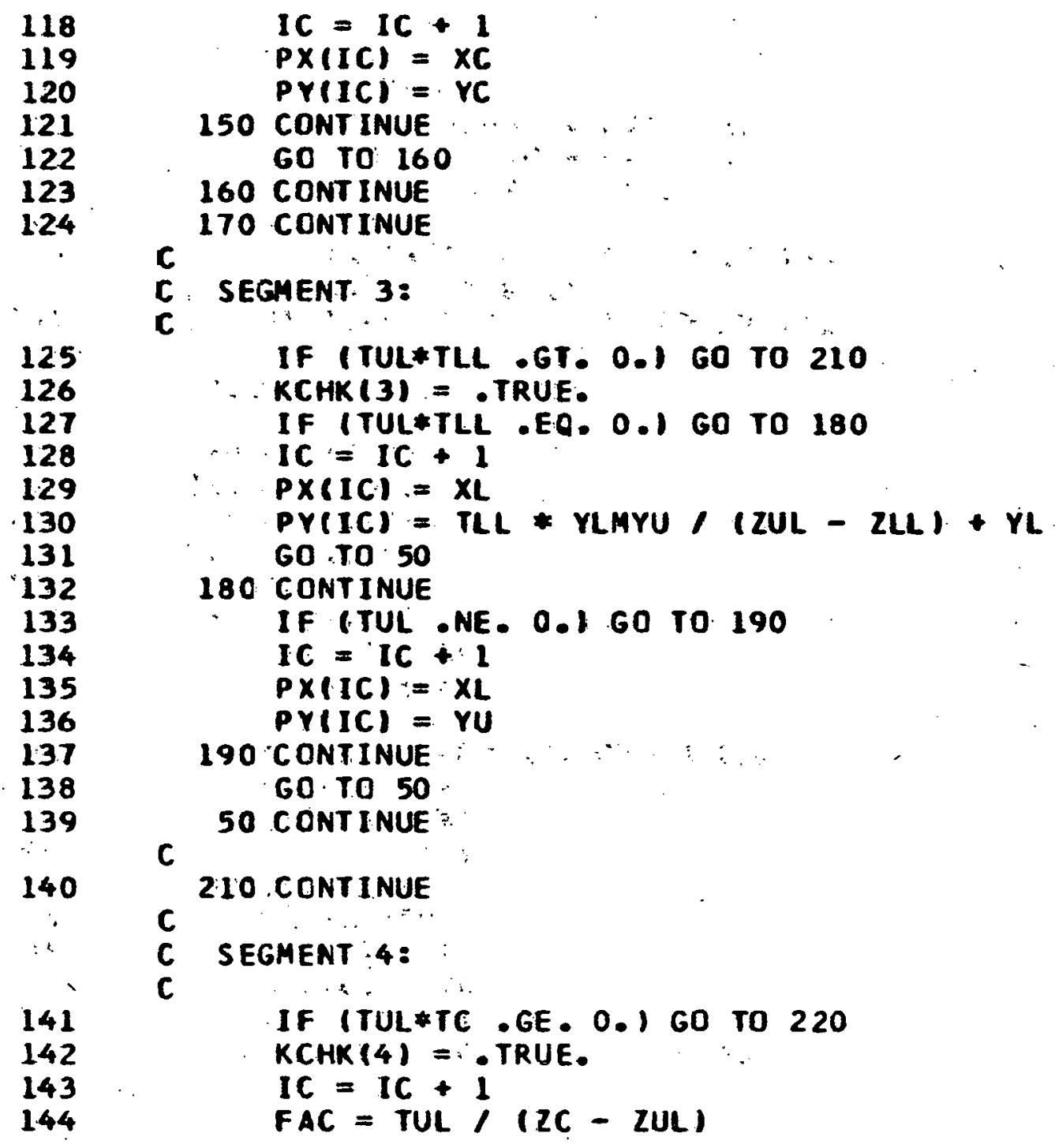




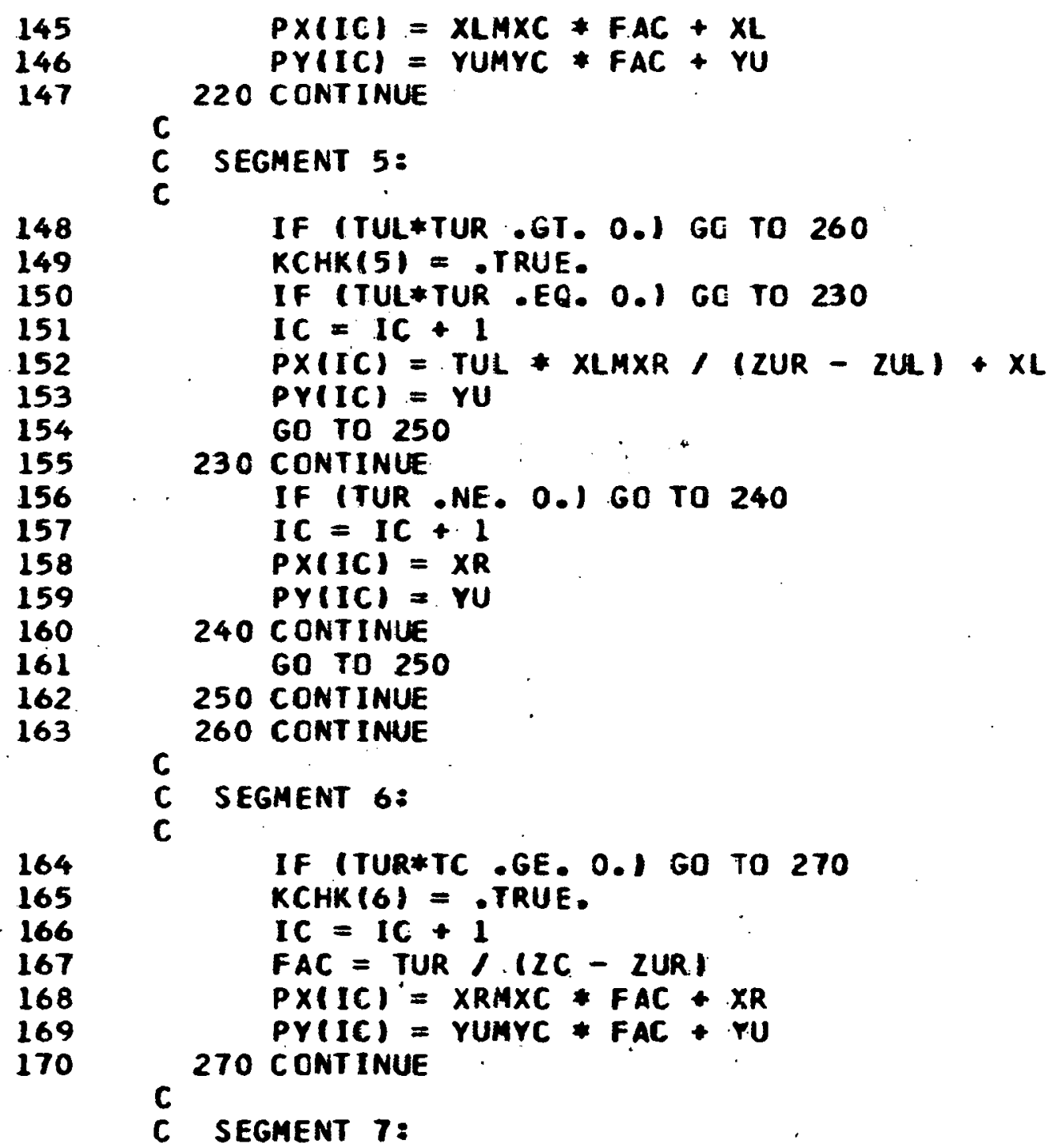


c

IF (TLR*TC . GE. O.) GO TO 290

KCHK $(8)=$.TRUE:

$I C=I C+1$

$F A C=T C /(Z C:-Z L R)$

$P X(I C)=X R A X C * F A C+X C$

$P Y(I C)=$ YLMYC * FAC + YC 290 CONT INUE

$C$ NOW DERIVE THE LINE SEGMENTS TO BE DRAWN FROM THE CONTENTS C OF THE PX AND PY ARRAYS.

C

IF IIC.LE. 1 ) 60 TO 410

IF (IC. GE. 6. OR. (IC-.EQ. 5 .AND. CENTER) 60 TO 360

C

IF ( .NOT. KCHK(8) GO TO 330

DO $320 \mathrm{~L}=1,7$

IF (.NOT. KCHK(L)) GO TO 310

PXIIC +1$)=\operatorname{PX}(1)$

c

$\operatorname{PY}(1 C+1)=\operatorname{PY}(1)$

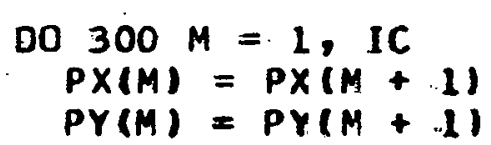


C

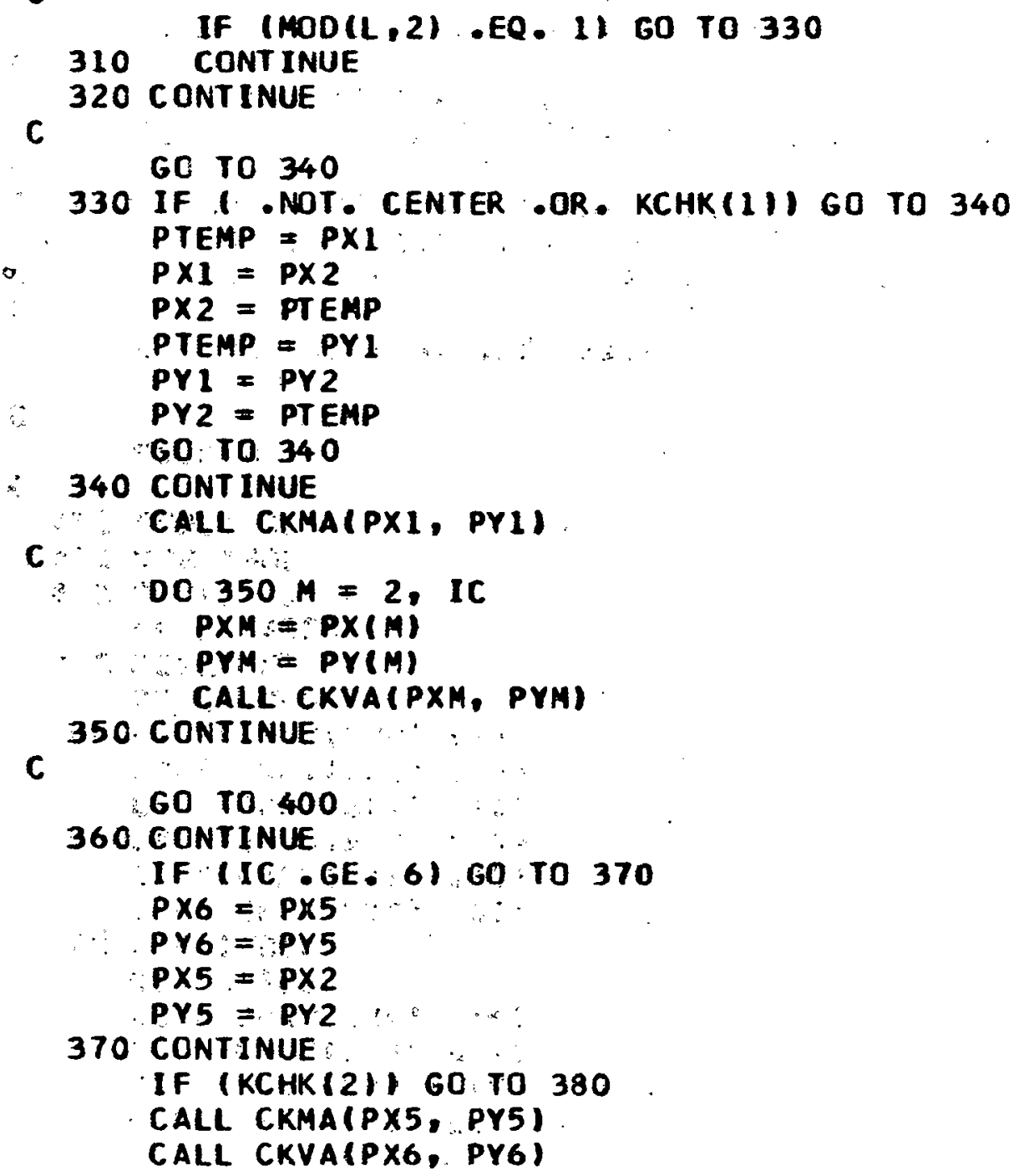


243

245

c

$\mathbf{C}$

$$
\text { RETURN }
$$

Subroutine CKmatpti, ptz)

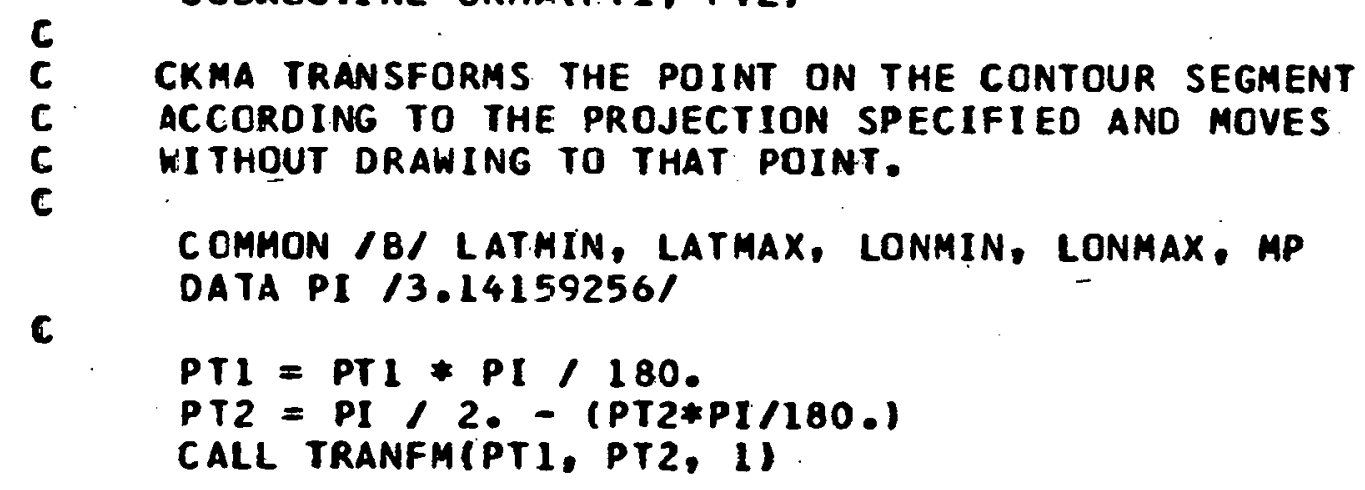


251

252

253

CALL PENUPSIPTI, PT2)

RETURN

END

ㅇํㅇ 
1

$c$
$C$
$c$
$C$

2

4

5

SUBROUTINE CKVAIPT1, PT2I

CKVA TRANSFORMS THE POINT. ON THE CONTOUR SEGMENT ACCORDING TO THE PROJECTION SPECIFIED AND DRAHS TO THAT POINT FROM THE LAST POINT.

COHMON /B/ LATMIN, LATHAX, LONMIN, LONMAX, MP DATA PI /3.14159256/

PTI $=$ PT $1 * P I / 180$.

PT2 = PI / 2. - (PT2*PI/180.)

CALL TRANFM(PT 1, PT2, 1$)$

CALL PENDNSIPT1, PT2I

RETURN

END 


\begin{tabular}{|c|c|}
\hline $\begin{array}{ll}\begin{array}{l}\text { NRC FORM } 335 \\
(7.77)\end{array} & \text { U.S. NUCLEAR REGULATORY COMMISSION } \\
& ; \quad \text { BIBLIOGRAPHIC DATA SHEET }\end{array}$ & $\begin{array}{l}\text { 1. REPOCAT NUMBER Assigned by DOCI } \\
\text { NUREG/CR-1 } 648\end{array}$ \\
\hline \multirow{2}{*}{$\begin{array}{l}\text { 4. TITLE AND SUBTITLE CAdovolume No. if eporoprisel } \\
\text { A Probăbilistic Evaluation of Earthquake Detection and } \\
\text { Location Capability for Illinois, Indiana, Kentucky, Ohio, } \\
\text { and. West Virginia }\end{array}$} & 2. (Leeve blenk) \\
\hline & 3. RECIPIENT'S ACCESSION NO. \\
\hline $\begin{array}{l}\text { 7. AUTHOR(S) } \\
\text { Frederick J. Mauk and Douglas H. Christensen }\end{array}$ & \begin{tabular}{|l|l|} 
5. DATE REPORT COMPLETED \\
MONTH JUNE & YEAA 980 \\
\end{tabular} \\
\hline \multirow{3}{*}{$\begin{array}{l}\text { 9. PERFORMING ORGANIZATION NAME AND MAILING ADDRESS Include Zip Code) } \\
\text { Seismological Observatory } \\
\text { Dept. of Geological Sciences } \\
\text { University of Michigan } 48109 \\
\text { Ann Arbor, MI } 48109\end{array}$} & \begin{tabular}{|c|c|}
\multicolumn{2}{|c|}{ DATE REPORT ISSUED } \\
MONTH \\
September & YEAR \\
\end{tabular} \\
\hline & 6. (Leave brenk) \\
\hline & 8. (Leeve brenk) \\
\hline \multirow{2}{*}{$\begin{array}{l}\text { 12. SPONSORING ORGANIZATION NAME AND MAILING ADDRESS (Include Zip Code) } \\
\text { Office of NuClear Regulatory Research } \\
\text { Division of Reactor Safety Research } \\
\text { U.S. Nuclear Regulatory Commission } \\
\text { Washington, D.C. } 20555\end{array}$} & 10. PROJECT/TASK/WORK UNIT NO. \\
\hline & $\begin{array}{l}\text { 11. CONTRACT NO. } \\
\text { NRC-04-76-192 } \\
\text { FIN NO. B5545 } \\
\end{array}$ \\
\hline $\begin{array}{l}\text { 13. TYPE OF REPORT } \\
\text { Technical }\end{array}$ & (Inclusive dowes) \\
\hline
\end{tabular}

15. SUPPLEMENTARY NOTES

14. (Leave bonk)

16. ABSTRACT Q00 words or loss)

Probabilistic estimations of earthquake detection and location capabilities for the states of Illinois, Indiana, Kentucky, Ohio and West Virginia are presented in this document. The algorithm used in these epicentrality and minimum-magnitude estimations is a version of the program NETWORTH by Wirth, Blandford, and Husted (DARPA Order No. 2551, 1978) which was modified for local array evaluation at the University of Michigan Seismological Observation. Estimations of earthquake detection capability for the years 1970 and 1980 are presented in four regional minimum m magnitude contour maps. Regional $90 \%$ confidence error ellipsoids are included for m magnitude events from 2.0 through 5.0 at $0.5 \mathrm{~m}$ unit increments. The close agreement between these predicted epicentral $90 \%$ confidence estimates and the calculated error ellipses associated with actual earthquakes within the studied region suggest that these error determinations can be used to estimate the reliability of epicenter location. 
UNITED STATES

NUCLEAR REGULATORY COMMISSION

WASHINGTON, D. C. 20555

OFFICIAL BUSINESS

PENALTY FOR PRIVATE USE, $\$ 300$
POSTAGE ANDFEES PAID U.S. NUCLEAR REGULATORY COMMISSION

\section{DO NOT MICROFILM COVER}

